\title{
Development of Advanced Electrochemical Emission Spectroscopy for Monitoring Corrosion in Simulated DOE Liquid Waste
}

\author{
by \\ Digby D. Macdonald, Brian M. Marx, Sejin Ahn, Julio de Ruiz, Balaji Soundararajan, \\ Morgan Smith, and Wendy Coulson \\ Center for Electrochemical Science and Technology \\ Pennsylvania State University \\ University Park, PA 16802 \\ digby@essc.psu.edu
}

Submitted to

Department of Energy Environmental Science Program

Grant No. DE-FG07-97ER62515 (Project 60219)

July 12, 2005 


\section{Table of Contents}

Executive Summary .................................................................................

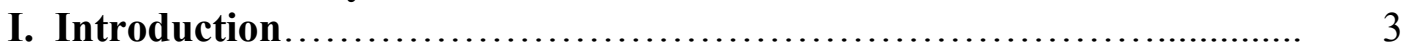

II. Background ...................................................... 5

II-1. Development of Corrosion Mechanisms...................... 5

II-2. Theoretical Study of Passivity Breakdown................................... 9

II-3. Crack Propagation Studies................................. 15

II-4. Electrochemical Emission Spectroscopy........................ 19

II-4.1. Generation of EC Noise from General and Localized Corrosion........................................ 19

II-4.2. Computer Simulation of Anodic/Cathodic Activity...... 20

II-4.3. Wavelet Analysis.................................... 22

II-5. Unification of the Deterministic and Statistical Approaches for

Predicting Corrosion Damage................................ 22

III. Research Progress............................................. 26

III-1. Continued Development of the Theory of Passivity............ 26

III-1.1a,b. Deriving Impedance Equations from PDM......... $\quad 26$

III-1.1c. XPS Work on Iron Oxide Film...................... 45

III-1.2. Obtaining Kinetic Information for Passive Iron via Curve-Fitting ................................... 47

III-1.3. Experimental Set-up to Obtain Data for DFA......... 50

III-2. The Role of Chloride Ion in Passivity Breakdown............. 52

III-2.1. Experimental Set-up................................ 52

III-2.2. Applying Mott-Schottky Analysis and EIS to Study Passivity Breakdown........................ 53

III-3. Crack Propagation Studies............................... 63

III-3.1. Experimental Set-up............................. 63

III-3.2. Identification of the Ideal Parameters for Describing Crack Propagation............................... 67

III-3.3. Frequency Domain Analysis for High Solution Concentrations................................. 68

III-3.4. Time Domain Analysis for Low Solution Concentrations................................... 74

III-3.5 Anisotropy of Stress Corrosion Cracking in AISI 4340 Steel.......................................... 78

III-3.6 Role of Crack Flanks in the Stress Corrosion Cracking of AISI 4340 Steel in $\mathrm{NaOH}$ Solutions........ 84

III-3.7 Role of Crevice - Darwinian Death of a Stress Corrosion Crack in the Presence of a Crevice........... $\quad 87$

III-4. Electrochemical Emission Spectroscopy...................... 94

III-4.1: Computer Simulation of the Anodic and Cathodic Activity 
III-4.2 Experimental Modeling of the Electrochemical

Environment.................................. 102

III-4.3 Wavelet Analysis (WA) of the EN Data.............. 108

III-4.4 Conclusions and Future Work......................... 160

III-5. Combining the Deterministic and Statistical Approaches

For Predicting Localized Corrosion Damage................... 163

III-5.1. Developing a Theoretical Basis for DFA.............. 163

III-5.2. Generalization of PDM to Describe Passivity

Breakdown................................... 173

III-5.3. Developing a Relationship Between the

Deterministic and Statistical Methods for Predicting the Progression of Corrosion Damage............... 179

IV. Publications in Peer Reviewed Journals/Conference Proceedings... 196

V. Presentations................................................. $\quad 198$

VI. Current Student Status......................................... 199 


\section{EXECUTIVE SUMMARY}

Various forms of general and localized corrosion represent principal threats to the integrity of DOE liquid waste storage tanks. These tanks, which are of a single wall or double wall design, depending upon their age, are fabricated from welded carbon steel and contain a complex waste-form comprised of $\mathrm{NaOH}$ and $\mathrm{NaNO}_{3}$, along with trace amounts of phosphate, sulfate, carbonate, and chloride. Because waste leakage can have a profound environmental impact, considerable interest exists in predicting the accumulation of corrosion damage, so as to more effectively schedule maintenance and repair.

The different tasks that are being carried out under the current program are as follows: (1) Theoretical and experimental assessment of general corrosion of iron/steel in borate buffer solutions by using electrochemical impedance spectroscopy (EIS), ellipsometry and XPS techniques; (2) Development of a damage function analysis (DFA) which would help in predicting the accumulation of damage due to pitting corrosion in an environment prototypical of DOE liquid waste systems; (3) Experimental measurement of crack growth rate, acoustic emission signals and coupling currents for fracture in carbon and low alloy steels as functions of mechanical (stress intensity), chemical (conductivity), electrochemical (corrosion potential, ECP), and microstructural (grain size, precipitate size, etc) variables in a systematic manner, with particular attention being focused on the structure of the noise in the current and its correlation with the acoustic emissions; (4) Development of fracture mechanisms for carbon and low alloy steels that are consistent with the crack growth rate, coupling current data and acoustic emissions; (5) Inserting advanced crack growth rate models for SCC into existing deterministic codes for predicting the evolution of corrosion damage in DOE liquid waste storage tanks; (6) Computer simulation of the anodic and cathodic activity on the surface of the steel samples in order to exactly predict the corrosion mechanisms; (7) Wavelet analysis of EC noise data from steel samples undergoing corrosion in an environment similar to that of the high level waste storage containers, to extract data pertaining to general, pitting and stress corrosion processes, from the overall data. 
The Point Defect Model (PDM) is directly applied as the theoretical assessment method for describing the passive film formed on iron/steels. The PDM is used to describe general corrosion in the passive region of iron. In addition, previous work suggests that pit formation is due to the coalescence of cation vacancies at the metal/film interface which would make it possible to use the PDM parameters to predict the onset of pitting [2]. This previous work suggests that once the critical vacancy density is reached, the film ruptures to form a pit. Based upon the kinetic parameters derived for the general corrosion case, two parameters relating to the cation vacancy formation and annihilation can be calculated. These two parameters can then be applied to predict the transition from general to pitting corrosion for iron/mild steels. If cation vacancy coalescence is shown to lead to pitting, it can have a profound effect on the direction of future studies involving the onset of pitting corrosion.

The work has yielded a number of important findings, including an unequivocal demonstration of the role of chloride ion in passivity breakdown on nickel in terms of cation vacancy generation within the passive film, the first detection and characterization of individual micro fracture events in stress corrosion cracking, and the determination of kinetic parameters for the generation and annihilation of point defects in the passive film on iron. The existence of coupling between the internal crack environment and the external cathodic environment, as predicted by the coupled environment fracture model (CEFM), has also been indisputably established for the AISI $4340 / \mathrm{NaOH}$ system. It is evident from the studies that analysis of coupling current noise is a very sensitive tool for studying the crack tip processes in relation to the chemical, mechanical, electrochemical and microstructural properties of the system. Experiments are currently being carried out to explore these crack tip processes by simultaneous measurement of the acoustic activity at the crack tip in an effort to validate the coupling current data. These latter data are now being used to deterministically predict the accumulation of general and localized corrosion damage on carbon in prototypical DOE liquid waste storage tanks. Computer simulation of the cathodic and anodic activity on the steel surfaces is also being carried out in an effort to simulate the actual corrosion process. Wavelet analysis of the coupling current data promises to be a useful tool to differentiate between the different corrosion 
mechanisms. Hence, wavelet analysis of the coupling current data from the DOE waste containers is also being carried out to extract data pertaining to general, pitting and stress corrosion processes, from the overall data which is bound to contain noise fluctuations due to any or all of the above mentioned processes

\section{INTRODUCTION}

The DOE currently stores about 253 million liters of high-level nuclear waste at the Hanford Reservation in Washington. The waste consists of a concentrated solution of $\mathrm{NaOH}, \mathrm{NaNO}_{3}, \mathrm{NaNO}_{2}$, and $\mathrm{NaCl}$, with other species such as $\mathrm{NaSO}_{4}, \mathrm{NaHPO}_{4}, \mathrm{NaAlO}_{2}$, and $\mathrm{NaF}$ also being present. Additionally, various radioactive species and "sludge", comprising a mixture of precipitated salts, are also present in the system. The temperature of the waste is typically $95^{\circ} \mathrm{C}-97^{\circ} \mathrm{C}$ and the $\mathrm{pH}\left(25^{\circ} \mathrm{C}\right)$ is greater than 12 . The waste is stored in a variety of single walled and doubled wall tanks manufactured from ASTM A537-Cl. 1, ASTM A515-Gr. 60, or ASTM A516-Gr. 60 steel. The single walled tanks were not stress relieved after welding, and hence contain weld heat-affected zones (HAZs) that are partially martensitic. These HAZs are susceptible to stress corrosion cracking (SCC) and/or hydrogen-induced fracture (HIC). Some of the single walled tanks are suspected to be leaking due to corrosion-induced penetrations of the walls.

The grades of steel used for the storage of DOE waste exhibit good corrosion resistance when in contact with alkaline solutions at temperatures below $100^{\circ} \mathrm{C}$. Of particular importance is the presence of chloride and sulfate ions in the waste, because these species are known to induce pitting corrosion on carbon and low alloy steels, amongst other alloys. Accordingly, general corrosion, pitting corrosion, and stress corrosion cracking are the most important degradation mechanisms. Although the general corrosion rate of iron in caustic environments is very low $\left(<0.1 \mathrm{~mm} / \mathrm{yr}\right.$ at $\left.100^{\circ} \mathrm{C}\right)$, the long storage times (100+ years) makes possible the accumulation of significant general corrosion damage. Exacerbating the corrosion problem is the presence of water radiolysis products, such as $\mathrm{H}_{2} \mathrm{O}_{2}, \mathrm{O}_{2}$, and $\mathrm{H}_{2}$, which may alter the corrosion potential and lead to the onset of specific failure processes, such as caustic and nitrate cracking. 
One of the major problems in ensuring tank integrity is the detection and monitoring of various forms of corrosion. In the case of general corrosion, the problem stems from the inherently high corrosion resistance of carbon steel in alkaline environments, which implies that the corrosion current density is low (typically $<1$ $\left.\mu \mathrm{A} / \mathrm{cm}^{2}\right)$. Because iron is highly passive, the noise in the current and potential, due to fluctuations in the local anodes and cathodes on the surface, is also very small (typically a few nano amperes and milli volts, respectively), making their accurate measurement difficult. Accordingly, any monitoring technique that is devised to monitor general corrosion from a remote location faces the challenge of transmitting very small signals from the sensors to the recording points without significant modification due to external interference (for example). On the other hand, localized corrosion processes, such as pitting corrosion and stress corrosion cracking, produce significant noise in both the potential and current, but because they are highly localized it is difficult, if not impossible, to predict a priori the exact locations at which they will occur and hence should be monitored. Accordingly, monitoring of these phenomena is best carried out using surveillance specimens that are configured to induce specific forms of localized corrosion.

After acquiring the potential and current noise fluctuations from the carbon steel containers, it is important to have suitable methods to extract the data pertaining specifically to general, pitting and stress corrosion processes from the overall data which contains noise fluctuations due to any or all of the above mentioned processes. Suitable methods are being developed to solve this problem.

Additionally, it is possible that at any given time the dominant corrosion mechanisms can be predicted by a suitably constructed computer simulation. Such a simulation would involve modeling the anodic and cathodic activity on the surface of the specimens and manipulating the rules governing the simulation until the EC noise resembled that of the experimental data set. Accordingly, simulation work is also currently being carried out. 


\section{BACKGROUND}

\section{II-1. Development of Corrosion Mechanisms}

General Corrosion occurs by the direct dissolution of a metal in the active state or by the dissolution of the passive oxide film in the passive state. In the latter case, which is the appropriate scenario for iron in DOE liquid waste, passive film dissolution is compensated for by growth of the oxide into the metal substrate, so that the corrosion rate is ultimately determined by the rate of dissolution of the oxide film and by the transmission of cations through the film. Accordingly, any model that is developed to predict the accumulation of damage due to general corrosion must address both the film dissolution and film formation processes that occur at the film/solution interface and at the metal/film interface, respectively. This issue has been addressed during the current program with the derivation of a new rate law for the growth of a passive film on a metal surface. The new law is based on the Point Defect Model (PDM) [1] for the growth and breakdown of passive films. The derivation of this rate law was shown in an earlier report and is not shown here. It is important to point out that this rate law depends on knowing the kinetic parameters for the reactions depicted by the PDM. Therefore, the focus of the current work on general corrosion is to obtain these kinetic parameters.

The goal of this portion of the project is to provide an assessment of the general and local corrosion of iron/steel. Knowledge of the kinetic parameters will lead to a much greater understanding of the general corrosion process occurring on iron/steels and will, ultimately, allow for the much more accurate prediction of failure. Damage Function Analysis (DFA) [2] will be implemented to predict damage accumulation due to pitting corrosion. Eventually, the kinetic parameters derived by the PDM will be incorporated into DFA. This combination will yield a deterministic/probabilistic model (based on Extreme Value Statistics) that can be used to predict failure in accordance with the pertinent conditions. Once developed, the PDM/DFA will be applied as a method to analyze data obtained by the monitoring of the electrochemical/environmental conditions such as current noise, $\mathrm{pH}$, aggressive ion concentration, and temperature. The end result will be the formation of an in-depth scientific method capable of monitoring the 
corrosion of iron/steel structures, containers, and vessels (in contact with solutions) for the purpose of predicting damage/failure.

High purity iron (Alfa Aesar-99.99\%) is being used in this portion of the work to extract the kinetic parameters for use in the PDM. To determine the kinetic parameters, the reactions involved in the corrosion process must be known (or assumed to be the ones involved). Without knowing the reactions occurring, it is impossible to account for the kinetic parameters governing the corrosion process. Extracting kinetic data without knowing/proposing which reactions are occurring would be like providing a destination time/distance without knowing the specific path being taken to the destination. By using pure iron, rather than steel, the number of possible reactions occurring simultaneously at the surface is greatly decreased (like decreasing the number of possible roads that can be taken to reach one's destination). For this reason, high purity iron is used in these experiments. Due to the possibility that some question may arise as to the validity of applying the kinetic data gathered for iron to steel, a discussion of this assumption follows.

The iron oxide formed on mild steel is believed to be responsible for the steel's passive characteristics (in the passive region), and not the oxides/chemical compounds formed by the other additives in the steel ( $\mathrm{C}, \mathrm{Mg}, \mathrm{Mn}$, etc.) [5]. Therefore, it is much simpler to account for the reactions occurring at the surface of the pure iron rather than the multitude of reactions possible on a steel surface. Although it is likely that the other elements in the steel could be involved in reactions occurring at the surface, their influence is considered to be very minor due to their very low concentrations. From a scientific point of view, it is beneficial to use high purity iron for the experimental work to eliminate any error that could be imparted to the data due to reactions involving the alloying elements. After collecting the kinetic parameters for iron, some experiments will be carried out on mild steel to ensure that the kinetic parameters derived for the iron are valid for steel. Previous fundamental work on the PDM lists the reactions assumed to be happening at the iron/oxide/solution interface [3].

Despite the fact that the fundamental reactions are known, a complication arises due to the fact that iron forms a double oxide layer comprised of an inner layer of $\mathrm{Fe}_{3} \mathrm{O}_{4}$ 
(magnetite) and an outer precipitation layer of $\mathrm{Fe}_{2} \mathrm{O}_{3}$ (hematite). It is widely accepted that the inner magnetite layer is responsible for the passive behavior of iron. However, the outer layer of hematite obstructs the inner layer from experimental observation.

Early work on this project revealed a method for removing the outer precipitation layer formed on iron, enabling the inner layer to be studied directly [3,4]. It has been shown that the addition of EDTA (chelating agent) to a borate buffer solution prevents the outer precipitation layer (hematite, $\mathrm{Fe}_{2} \mathrm{O}_{3}$ ) from forming on iron in solution. By removing the precipitation layer, the inner $\mathrm{Fe}_{3} \mathrm{O}_{4}$ (magnetite) can be studied directly. The early fundamental work on the PDM reactions for iron and the work on the removal of the precipitation layer made it possible to experimentally obtain kinetic parameters. This makes it possible to build a comprehensive database of kinetic parameters as functions of temperature, potential, and $\mathrm{pH}$. A database containing the kinetic parameters allows us to analyze electrochemical data by the PDM and make predictions concerning damage/failure of iron/steels for various conditions.

The next step is to derive the equations for the impedance and thickness of the iron oxide film $\left(\mathrm{Fe}_{3} \mathrm{O}_{4}\right)$ as described by the PDM. Once the equations are derived, experiments can be performed to measure the impedance and the film thickness. Figure II-1.1 shows the experimental setup in which a Solartron 1250 Frequency Response Analyzer (FRA) is being used for Electrochemical Impedance Spectroscopy (EIS), a Solartron 1286 potentiostat is being used for potentiostatic control, and a Sopra scanning wavelength ellipsometer is being used to measure the film thickness. DataFit software will be used to fit the impedance equation to the data. Once a reasonable fit is obtained, the kinetic parameters can be extracted from the simulation. These parameters will then be used to predict the impedance of films as functions of temperature, $\mathrm{pH}$, and potential. Further experiments will be performed to verify these predictions. 


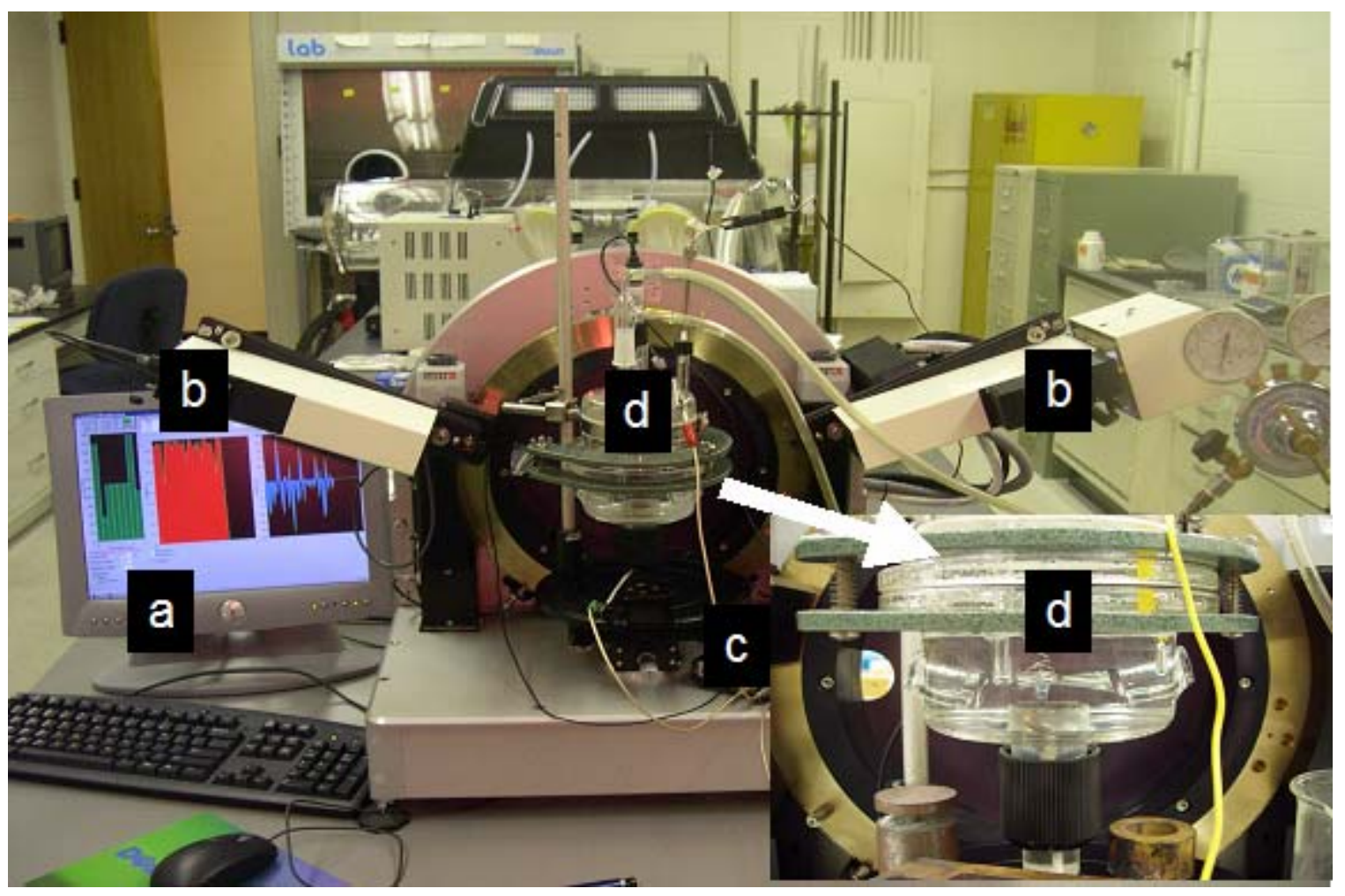

Figure II-1.1 Picture of the spectroscopic ellipsometer and associated systems. The PC used to collect the ellipsometric and impedance data (a). Two arms of ellipsometer-used to detect the changes in reflected light (b). Potentiostat/Frequency Response Analyzer (FRA) for controlling the potential at the working electrode and measuring AC impedance (c). The cell containing the electrochemical set-up (d).

This report separates the work into sections under the research progress heading (III. Research Progress in the Table of Contents). Each section describes the tasks being performed, the immediate goals of each, and what work has been completed. For the general/pitting corrosion work, the first section discusses the experimental methodology used to obtain impedance and ellipsometry data that will be analyzed to extract the kinetic parameters required by the PDM for making predictions. Also in this section is a brief description of some XPS work that has been performed to quantify the relative amounts of the iron species in the film. The final section describes the experimental setup being developed to obtain pitting data for use in DFA. 


\section{II-1. References}

1. D.D. Macdonald, M. Uriquidi-Macdonald, J. of Electrochemical Society, 137, 2395 (1990).

2. G. Engelhardt, D.D. Macdonald, Corrosion, 54, 469 (1998).

3. J. Liu, D.D. Macdonald, J. of Electrochemical Society, 148, B425 (2001).

4. E. Sikora, D.D. Macdonald, J. of Electrochemical Society, 147, 4087 (2000).

5. A. Valeria, C.M.A. Brett, Electrochimica Acta, 47, 2081 (2002).

\section{II-2. Theoretical Study of Passivity Breakdown}

The passive current density for most reactive transition metals and their alloys (e.g. $\mathrm{Fe}, \mathrm{Ni}, \mathrm{Cr}, \mathrm{Ti}, \mathrm{Zr}$ ) in contact with ambient aqueous environments is of the order of 1 $\mu \mathrm{A} / \mathrm{cm}^{2}$ to $0.01 \mu \mathrm{A} / \mathrm{cm}^{2}$, corresponding to a metal penetration rate of about $10 \mu \mathrm{m} /$ year to $0.1 \mu \mathrm{m} / \mathrm{year}$. Penetration rates of this magnitude do not represent a significant threat to the integrity of industrial or infra-structural systems over the design lifetime, except for special cases (e.g. ball bearings) where dimensional stability is critical for proper operation and function. Unfortunately, passive films do not afford complete protection of the substrate metal or alloy, in that certain species (e.g., $\mathrm{Cl}^{-}$) induce passivity breakdown. This results in various forms of localized corrosion, such as pitting attack and stress corrosion cracking. Localized corrosion processes can result in the rapid penetration of a cavity (pit or crack) into the metal substrate, thereby leading to premature failure. Indeed, corrosion in general, of which localized corrosion is a major component, is estimated to cost an industrialized country more than $4 \%$ of the GNP (approximately $\$$ 400 billion for the U.S. in 2002), rendering it one of the most important costs in operating industrial systems. However, the "cost of corrosion" never appears in a corporate balance sheet, so that the cost is largely hidden from the shareholder and consumer alike.

Because of its practical and scientific importance, passivity and passivity breakdown have been studied intensively since the time of Faraday (1830s) and numerous theories and models have been proposed to explain the phenomen [2,6-9]. However, because of the lack of in situ experimental techniques having the required spatial and temporal resolution, few atomic scale details of the processes that occur within the passive film have emerged from these studies. Nevertheless, it is now generally agreed that the barrier oxide layer is a highly defective structure, with the point 
defects being metal and oxygen vacancies and metal interstitials. The identity of the principal defect depends upon the relative energies of formation upon the system [1].

The PDM provides an analytical description of the growth and breakdown of passive films on reactive metal surfaces [1]. The fundamental basis of the general PDM is illustrated in Figure II-2.1, in which the elementary reactions that lead to the generation and annihilation of point defects at the interfaces (Reactions $1-6$ ), together with film dissolution (Reaction 7), are displayed. Of particular importance in the present discussion are Reactions 4 and 1, which represent the generation and annihilation of cation vacancies at the barrier layer/solution and metal/barrier layer interfaces, respectively, resulting in a flux of cation vacancies across the film as indicated.

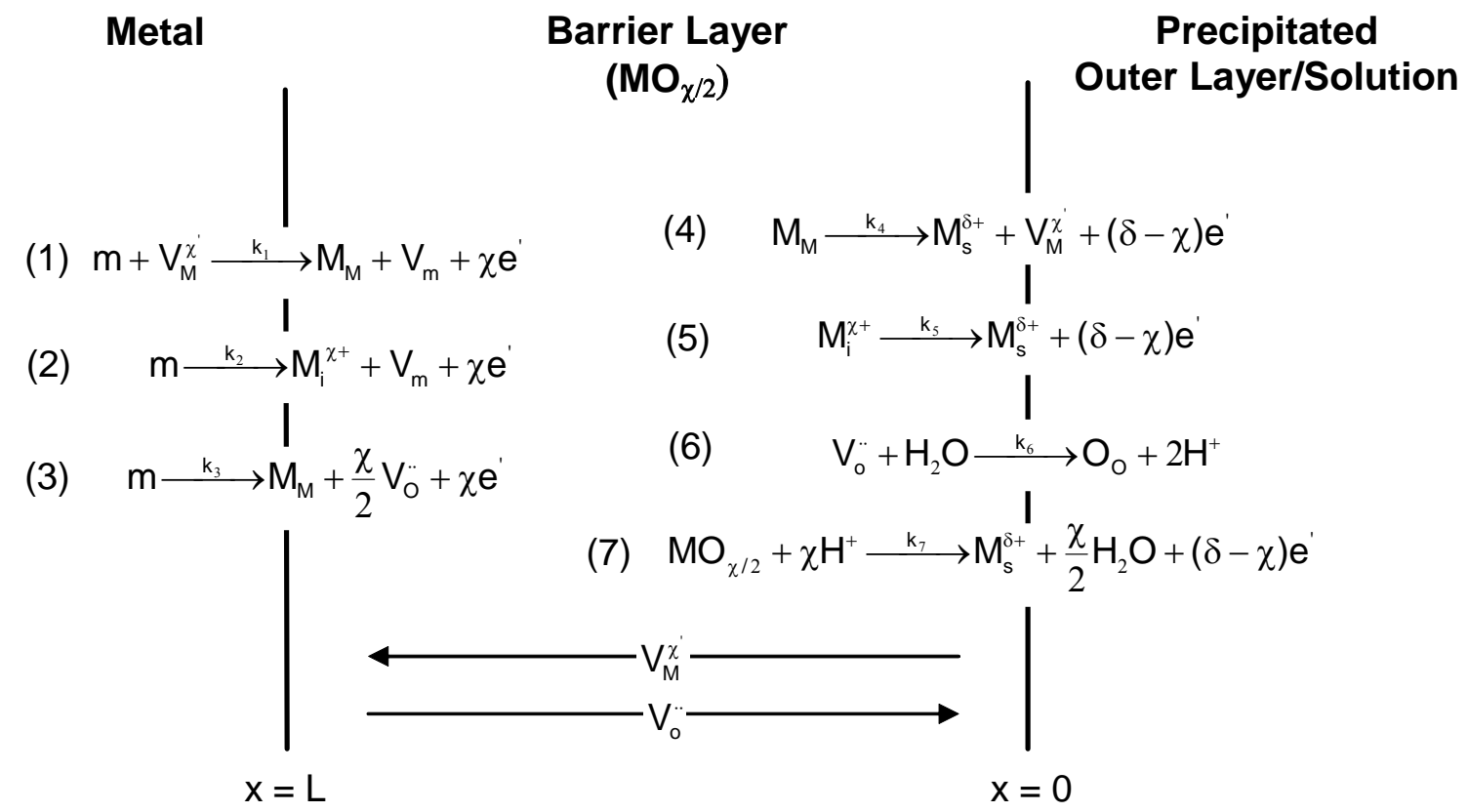

Figure II-2.1 Schematic of physico-chemical processes that occur within a passive film according to the Point Defect Model. $\mathrm{m}=$ metal atom, $V_{M}^{\chi^{\prime}}=$ cation vacancy, $\mathrm{M}_{\mathrm{M}}=$ cation in cation site, $M_{i}^{\chi^{+}}=$cation in interstitial site, $V_{O}^{\bullet}=$ anion vacancy, $M_{s}^{\chi^{+}}=$cation in outer layer/solution, $O_{O}=$ oxygen ion in anion site, $M O_{\chi / 2}=$ stoichiometric barrier layer oxide. Cation vacancies are produced at the film/solution interface, but are consumed at the metal/film interface. Likewise, anion vacancies are formed at the metal/film interface, but are consumed at the film/solution interface. Consequently, the fluxes of cation vacancies and anion vacancies are in the directions indicated [1].

The PDM postulates that, if the cation vacancies arriving at the metal/barrier layer interface cannot be annihilated by Reaction 1, then the vacancies will condense to 
form a "vacancy condensate" that effectively separates the film from the substrate metal (Figure II-2.2), with vacancy condensation continuing to occur at the periphery, thereby resulting in growth of the blister. Separation of the barrier layer from the metal results in cessation of growth of the barrier layer into the substrate metal or alloy via Reaction 3, Figure II-2.1. However, the barrier layer continues to dissolve at the barrier layer/solution (outer layer) interface [Reaction 7, Fig. II-2.1], thereby resulting in the local thinning of the barrier layer [Fig. II-2.2(c)]. Thinning continues until the local growth stresses within the barrier layer result in the rupture of the "cap" over the vacancy condensate, finally resulting in a passivity breakdown event, as shown schematically in Figures II-2.2(d) and (e). The nucleus may undergo "prompt repassivation" in which case it represents a "meta stable pitting event, or it may stabilize to form a "stable" pit that will grow to induce macroscopic damage. Eventually, the stable pit will also die via delayed repassivation, which arises for a number of reasons, including the limitation of the resources that are available on the external surfaces and competition between neighboring pits, as outlined previously [1]. The ratio of the probability of formation of a stable pit to the probability of formation of a stable and meta stable pit is known as the survival probability. This quantity is readily measured and is found to have a value of about $10^{-4}$ for pitting on Type 304 stainless steel [10]. Stable pitting is indeed a rare (but very damaging) event. 


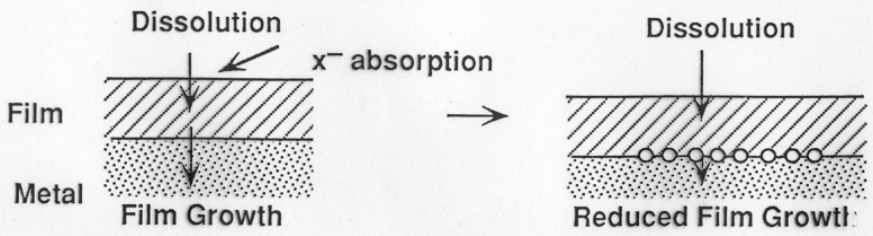

Film Growth

(a) Steady State (b) Vacancy Condensation

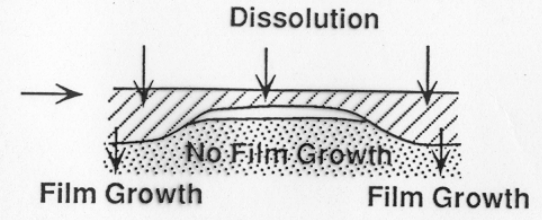

(c) Local Film Detachment

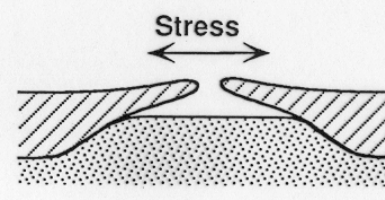

(d) Film Rupture

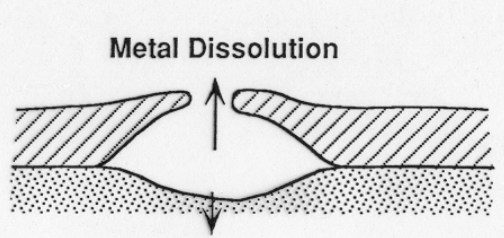

(e) Pit Growth
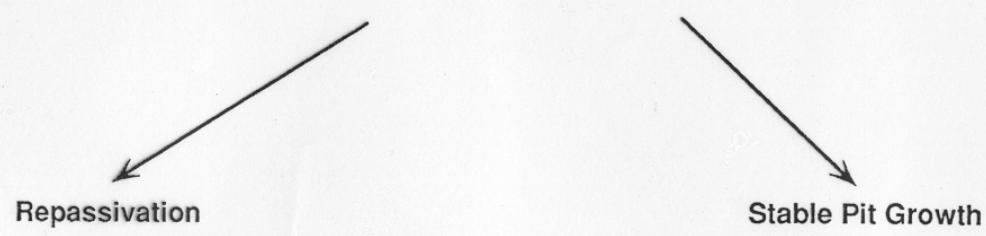

Figure II-2.2 Cartoon outlining various stages of pit nucleation according to the Point Defect Model [1].

This basic picture of the sequence of events in passivity breakdown has received strong experimental support, ranging from the direct observation of blister formation as the precursor to passivity breakdown [11,12], which accounts for the photo-inhibition of passivity breakdown [13], to correctly predicting the dependencies of the critical breakdown voltage and induction time on various independent variables \{e.g., $\left[\mathrm{Cl}^{-}\right], \mathrm{pH}$, and potential ( $\mathrm{t}_{\text {ind }}$ only) $\}[1]$, to name but a few of the successful applications of the model. A particularly convincing test of the PDM is afforded by studying the voltage sweep rate dependence of the apparent breakdown voltage, because the quantity that is derived (the areal concentration of vacancies in the condensate) can be compared with the value calculated from structural considerations. Studies of this type that have been performed on nickel [14] and aluminum [15] have found excellent agreement between theory and experiment. Implicit in the model is the concept that aggressive anions, such as $\mathrm{Cl}^{-}$, are able to enhance the flux of cation vacancies through the barrier layer, such that 
under favorable conditions (voltage, $\mathrm{pH},\left[\mathrm{Cl}^{-}\right]$) vacancy condensation will occur at the metal/barrier layer interface and hence passivity breakdown will ensue. Two mechanisms have been proposed for the impact of an aggressive anion on the cation vacancy flux, both of which envision the initial absorption of the anion into a surface oxygen vacancy (Figure II-2.3): (1) Schottky pair formation [16], and (2) cation extraction [1]. Both of these possibilities are represented in Figure II-2.3 and both lead to expressions of identical functional form for the critical breakdown voltage and induction time.

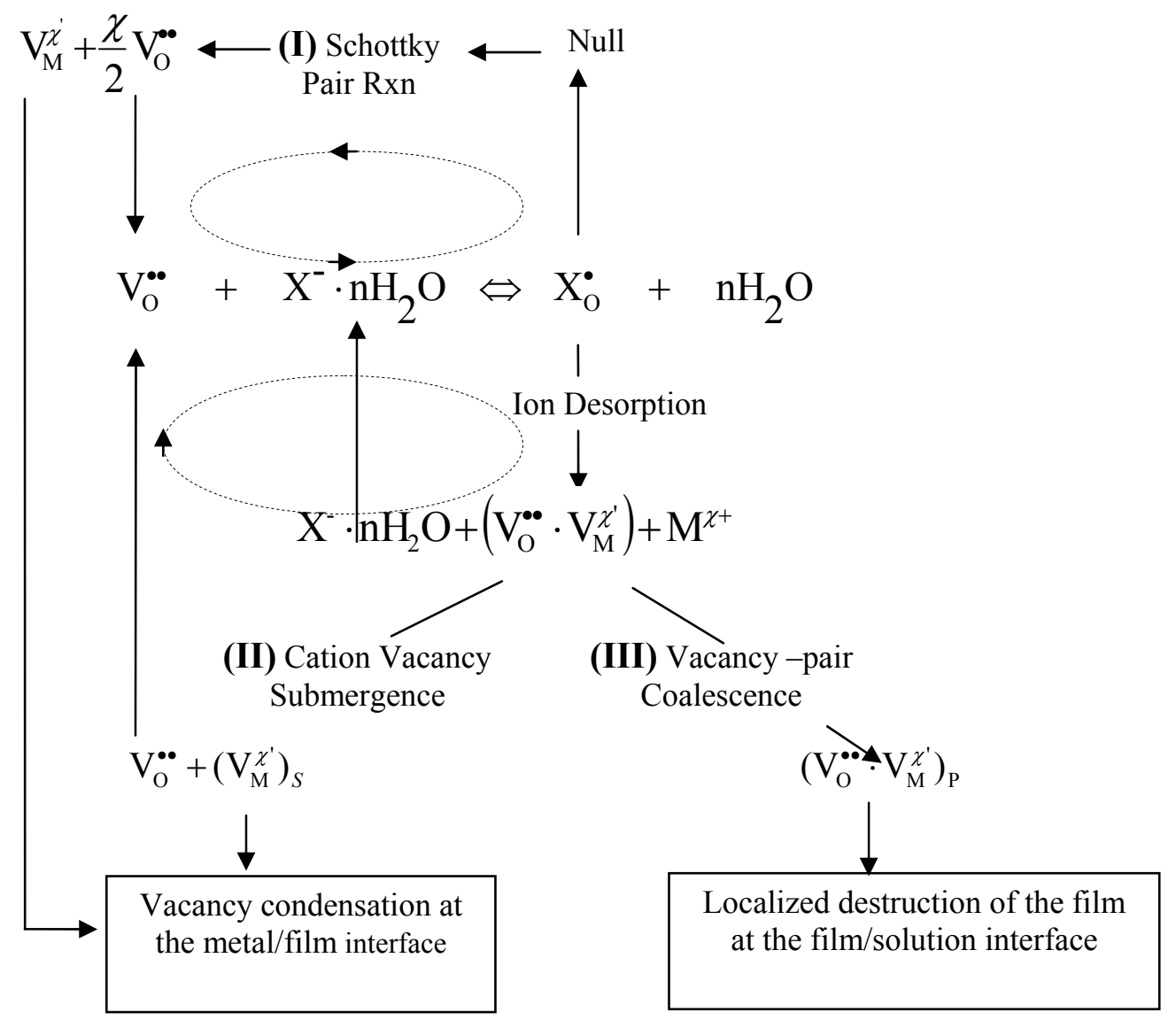

Figure II-2.3 Postulated reactions for cation vacancy generation at the barrier layer/solution interface according to the Point Defect Model [1]. The broken lines indicate the direction of the reactions that are postulated to be responsible for the generation of cation vacancies at the barrier layer/solution interface. Note that the occurrence of the reactions leads to the regeneration of oxygen vacancies and chloride ion and that if vacancy condensation occurs at the periphery of the blister then adsorption of chloride will occur on the outer surface of the barrier layer at the same location, as observed by Bargeron and Givens [10] for passivity breakdown on aluminum. 
Accordingly, it has not been possible to choose between the two possibilities upon the basis of measured dependencies of $V_{c}$ and $t_{i n d}$ on the system independent variables. It is important to note that both mechanisms lead to the regeneration of oxygen vacancies at the barrier layer/solution interface and the regeneration of chloride ions, which is then available to reabsorb into the regenerated oxygen vacancies, to continue the process. This process can only occur above (perpendicular to the surface) the location of cation vacancy condensation, and hence accounts for the important observation of Bargeron and Givens [11] on passive aluminum that a chloride ring on the surface expands at the periphery of the blister as the blister grows.

This section describes both the experimental characterization of changes in the vacancy structure of the barrier layer upon exposure of a passive surface to chloride ion and the process of ascertaining the exact nature of the cation vacancy generation reaction that occurs at the barrier layer/solution interface. Passive nickel was chosen for this study, because the passive film formed on nickel is a p-type semi conductor, indicating that the majority defect is the cation vacancy. Thus, any impact that chloride ion has on the concentration of the cation vacancy in, and the flux across, the barrier layer, the increase of which are postulated in the PDM to be responsible for passivity breakdown, should be readily detected using Mott-Schottky analysis. Specifically, the work was designed to detect changes in cation vacancy concentration in the passive film upon absorption of chloride into surface oxygen vacancies and to explore the nature of any cation vacancy generation process that may occur at the barrier layer/solution interface.

In this communication, we assess the impact that chloride ion, which is known to induce passivity breakdown in many metals and alloys, has on the electronic and point defect structures of the passive film on nickel. The principal findings are as follows:

- We show by Mott-Schottky analysis that, as the concentration of $\mathrm{Cl}^{-}$in $\mathrm{pH} 8.5$ borate buffer solution increases, the concentration of metal vacancies $\left(V_{M}^{\chi^{\prime}}\right)$ in the passive film on $\mathrm{Ni}$ also increases.

- The addition of $\mathrm{Cl}^{-}$to $\mathrm{pH} 8.5$ borate buffer solution after passivity has been established in the absence of $\mathrm{Cl}^{-}$also results in an increase in the concentration of 
metal vacancies $\left(V_{N i}^{\chi^{\prime}}\right)$ in the passive film.

- Finally, by using electrochemical impedance spectroscopy to interrogate the point defect generation and annihilation reactions that occur at the metal/film and film/solution interfaces, we show that the observed increase in cation vacancy concentration in the passive film is due to chloride catalyzed ejection of cations from the film/solution interface.

These findings are not consistent with chloride-catalyzed film dissolution and chloride penetration mechanisms for passivity breakdown, and they are consistent with the Point Defect Model.

\section{II-2 References}

1. D. D. Macdonald, Pure Appl. Chem., 71, 6, 951 (1999).

2. H. H. Uhlig, Passivity of Metals (R. P. Frankenthal, J. Kuger, eds), p.1. The Electrochemical Soc., Princeton, NJ (1978).

3. K. Ismail, E. Sikora, and D. D. Macdonald, J. Electrochem. Soc., 145, 3141 (1998).

4. E. Sikora and D. D. Macdonald, J. Electrochem. Soc., 147(11), 4087 (2000).

5. J. Liu and D. D. Macdonald, J. Electrochem. Soc., 148, B425 (2001).

6. R. P. Frankenthal and J. Kruger (eds.), Passivity of Metals, The Electrochemical Society, Princeton, NJ (1978).

7. M. Fromont (ed.) Passivity of Metals and Semiconductors, Elsevier Scientific, Amsterdam, (1983).

8. D. D. Macdonald, J. Electrochem. Soc., 139, 3434 (1992).

9. N. Sato, Passivity of Metals (R. P. Frankenthal, J. Kuger, eds), p.29. The Electrochemical Soc., Princeton, NJ (1978).

10. D. Williams, J Stewart and P. H. Balkwill, Corr. Sci., 36, 1213 (1994).

11. C. B. Bargeron and R. B. Givens, Corrosion, 36, 618 (1980).

12. D. J. Ellerbrock and D. D. Macdonald. Materials Science Forum, 185-188, 927 (1995).

\section{II-3. Crack Propagation Studies}

Perhaps the greatest, catastrophic failure mode that could befall DOE Liquid Waste storage tanks is stress corrosion cracking; specifically, caustic cracking (CC). This process usually occurs in high strength carbon or low alloy steels, in the heat affected 
zones adjacent to welds in these materials. In any event, crack propagation rates can be very high, thus leading to wall penetration soon after initiation. Despite the obvious importance of $\mathrm{CC}$ in any industry that handles concentrated hydroxide solutions (e.g., extraction of alumina from bauxite $[1,2]$ ) little is known of the mechanistic details of this crack propagation process.

Failure by caustic cracking of the carbon steel storage tank must be considered as one of the principal threats to tank integrity. An enormous literature exists on the environment assisted cracking (EAC) of carbon steels in caustic solutions, but the work is almost entirely phenomenological in nature. For example, it is not always clear whether crack propagation occurs via anodic dissolution or HIC, or a combination of both, but the bulk of the evidence suggests that HIC is the fracture mechanism. Thus, it has long been observed that cracks propagate via discrete events and this is often taken as being indicative of the role of hydrogen [4]. Thus, hydrogen is postulated to produce a brittle matrix at the crack tip that undergoes periodic fracture to produce intermittent crack advance [4]. HIC occurs with a fracture dimension that is determined by the time between events and the diffusion length of hydrogen in the matrix (typically a few microns). SCC may also occur by cyclical slip/dissolution/ repassivation $[5,6]$ and hence exhibits periodic advance, but the slip dimension should be of the order of some low multiple of the Burger's vector (a few nanometers) and is unlikely to give rise to the abrupt transient in current that has been found in this study. Thus, from a mechanistic viewpoint, knowledge of the dimension of the fracture event is most important, for it may provide a means of differentiating between anodic dissolution and hydrogen-induced fracture. However, quantitative knowledge of the mechanism(s) of caustic cracking of carbon steel in highly concentrated alkaline solutions is quite poor. Therefore, emphasis in this part of our work is on the fundamental aspect of caustic cracking mechanisms by using electrochemical emission spectroscopy (EES) on the coupling current that flows between the crack and the external surfaces to ascertain the dimension of the brittle micro-fracture events that occur during crack propagation. The well established acoustic emission technique (AET) has also been used to simultaneously monitor the crack propagation. 
Acoustic emission (AE) is the transient elastic radiation produced by many microscopic deformation and fracture processes in materials. When this elastic radiation contacts a surface, it produces a displacement that can be detected and measured by a suitable sensor. AE technology involves the use of ultrasonic transducers $(20 \mathrm{KHz}-1$ $\mathrm{MHz}$ ) to listen for the sounds of failure occurring in materials and structures. Acoustic emission sensors, which are usually made from piezoceramics, convert displacement to voltage which is amplified and subsequently stored or analyzed. Typical AE signals appear as an oscillating voltage that quickly rises to a peak value, and then slowly decays to the background noise level.

Acoustic emission has been detected in many materials during elastic and plastic deformation and fracture. Possible sources of these elastic waves include the rapid, collective motion of a large number of dislocations, inclusion and precipitate fracture or pull-out of the matrix, lüder band propagation in iron alloys, and rapid, brittle crack extension. Because many of these processes occur during sub-critical crack growth in materials, acoustic emission has a potential to be a monitor of crack initiation and propagation in engineering components [7-14]. The acoustic emission technique (AET) is a very useful tool for monitoring stress corrosion cracking, which is evident from the vast literature that is available [7, 9-14]. In the current research, AET is being employed to monitor the stress corrosion crack growth. Apart from crack growth studies, AET is an effective tool to detect crack initiation and crack depth, the details of which are given in the results section. Results from these experiments can be effectively used to corroborate the results obtained from EES.

In order to carry out the study in a parametric manner, the simple $\mathrm{NaOH} / \mathrm{H}_{2} \mathrm{O}$ electrolyte system was used. A high-strength, low-alloy steel (AISI 4340 [UNS G43400 (1)]) was chosen for the test material and was heat-treated to represent the HAZ adjacent to welds in DOE storage tanks. Methods have been developed to measure the electrochemical emissions in the coupling current from growing cracks in a high-strength, low alloy steel. These techniques are expected to provide fundamental information on the mechanisms of crack propagation. The method used is to measure the coupling current 
that flows between the crack and the external surface, as a crack propagates through the steel (Figure II-3.1).

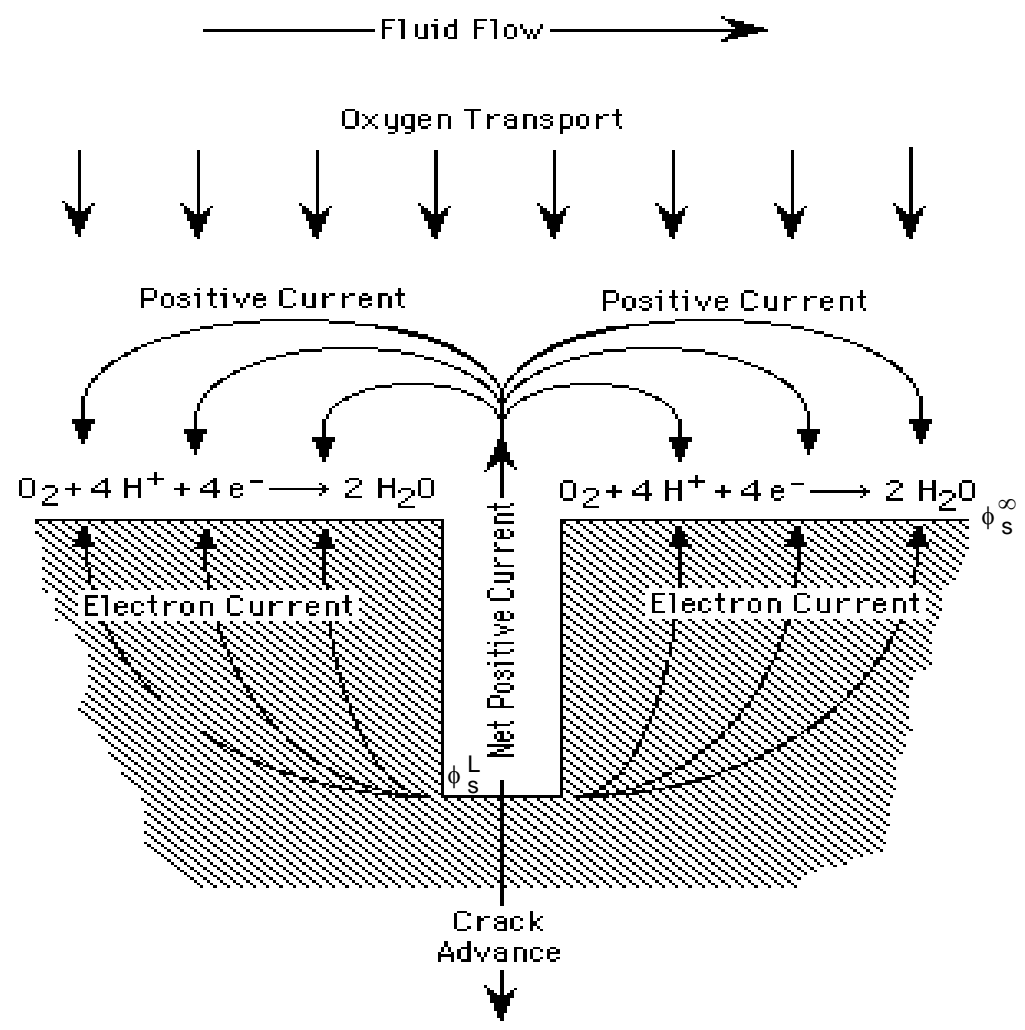

Figure II-3.1. Schematic of the origin of the coupling current in stress corrosion cracking. The coupling current is required by the differential aeration hypothesis for localized corrosion and the conservation of charge requires that the electron current flowing through the substrate from the crack to the external surface must be equal to the positive ionic current flowing through the solution from the crack to the external surface.

A coupling current of appropriate magnitude is required by the differential aeration hypothesis to satisfy charge conservation in the system [15]. Previous work on SCC in Type 304 stainless steel ([UNS 30400] SS) in high-temperature aqueous systems shows that the noise in the coupling current can be attributed to brittle micro-fracture events that have a dimension on the order of $3 \mu \mathrm{m}$ and that these fracture events occur in "packages" of 4 to 13, corresponding to the grain size of the steel [6]. The present work seeks to determine whether similar events can be detected in the coupling current that is produced in the fracture of high strength, low-alloy steels in simulated DOE waste environments. Simultaneous monitoring of the acoustic emission signals would help to 
verify if the individual transients in the coupling current actually correspond to a microfracture event. Detection and characterization of the noise in the coupling current is expected to provide mechanistic information of unprecedented value.

\section{II-3 References:}

1. H.H. Le, E. Ghali, Corrosion Science, 35, n 1, 435 (1993).

2. R.K. Singh Raman, B.C. Muddle, Materials Science and Technology, 19, n 5, $642(2001)$

3. G. L. Edgemon, M. J. Danielson, and G. E. C. Bell, J. Nuclear Materials, 245, 201 (1997).

4. M. P. Manahan, Sr, D. D. Macdonald and A. J. Peterson, Jr., Corros. Sci, 37, 189 (1995).

5. D. A. Vermilyea and R. B. Diegle, Corrosion, 32, 26 (1976).

6. J. C. Scully, Corros. Sci., 20, 997 (1980).

7. D.J. Mills, and S. Mabbutt, Prog. Org. Coat, 39, 41 (2000)

8. R.H. Jones, M.A. Friesel, and W.W. Gerberich, Met trans A, 20A, 637, (1989)

9. A. Berkovits and D. Fang, Engg. Fra. Mech, 51, 401 (1995)

10. F. Ferrer, E. Schille, D. Verardo and J. Goudiakas, JOM, 37, 2707 (2002)

11. K.Y. Sung, I.S. Kim and Y.K. Yoon, Scripta Materialia, 37, 1255 (1997)

12. B. Knowlton, A.M Siddiqui and S. Jihan, British Corrosion Journal, 32, 249 (1997)

13. R.H. Jones, M.A. Friesel and R. Pathania, Corrosion, 47, 105 (1991)

14. N.K. Mukhopadyay, G. Sridhar, N. Parida, S. Tarafder and V.R. Ranganath, Engineering Failure Analysis, 6, 253 (1999)

15. D. D. Macdonald and M. Urquidi-Macdonald, Corros. Sci., 32, 51 (1991)

\section{II-4 Electrochemical Emission Spectroscopy}

\section{II-4.1 Generation of EC Noise from General and Localized Corrosion}

Currently an electrochemical cell is being constructed for the collection of EC noise from general and localized corrosion. The cell's purpose is to model the environment inside the waste storage containers. The cell simulates the electrochemical environment and the temperature of the containers such that electrochemical noise data relating to general and localized corrosion of the type from the actual containers is generated. This electrochemical noise is collected for later analysis along with other types of corrosion noise data. 


\section{II-4.2 Computer Simulation of Anodic and Cathodic Activity}

The EC noise collected from the electrochemical cell is also being used to further the development of the model describing corrosion in the storage containers. It is the basis for computer modeling of the anodic and cathodic activity on the surface of the steel specimens. In the simulation, the surface of the sample is represented by a grid of possible sites. Initially a completely random arrangement of anodic and cathodic sites is produced, figure II-3.1.

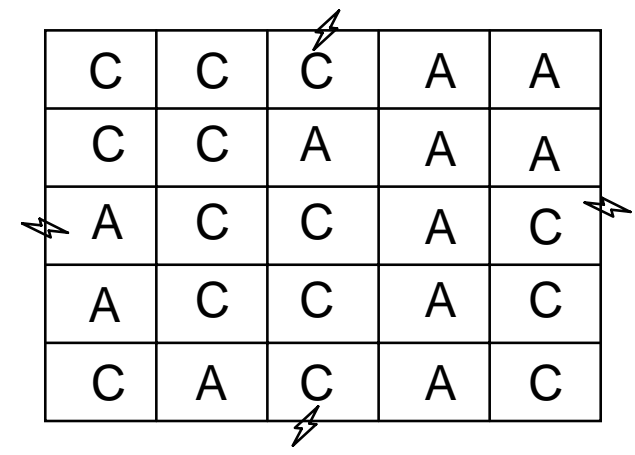

Figure II-3.1. Random anodic and cathodic sites

Whether an anodic site remains anodic or switches to a cathodic state from moment to moment is also initially completely random. The total number of anodic and cathodic sites is regulated by conservation of charge, thus it is always necessary to maintain charge equality across the entire simulated specimen surface. The EC processes are modeled mathematically by the following equations:

Anodic process: $\mathrm{I}_{\mathrm{a}}=\mathrm{N}_{\mathrm{a}} \mathrm{i}_{\mathrm{o}, \mathrm{a}} \mathrm{e}^{\wedge}\left(\mathrm{E} / \mathrm{b}_{\mathrm{a}}\right)$

Cathodic process: $I_{c}=N_{c} i_{o, c} e^{\wedge}\left(E / b_{c}\right)$

Where $\mathrm{N}_{\mathrm{a}}, \mathrm{N}_{\mathrm{c}} \quad$ : initial number of anodic and cathodic sites, respectively $\mathrm{i}_{\mathrm{o}, \mathrm{a}}$, io,c : initial anodic and cathodic current, respectively

E : potential $b_{a}$ and $b_{c}$ : anodic and cathodic Tafel slopes, respectively

$\Delta \mathrm{I}$ can be derived from these equations to be

$$
\Delta \mathrm{I}=\mathrm{i}_{\mathrm{o}, \mathrm{a}} \mathrm{e}^{\wedge}(\mathrm{E}+\Delta \mathrm{E}) / \mathrm{b}_{\mathrm{a}}-\mathrm{i}_{\mathrm{o}, \mathrm{c}} \mathrm{e}^{\wedge}-(\mathrm{E}+\Delta \mathrm{E}) / \mathrm{b}_{\mathrm{c}}
$$

But when $\Delta \mathrm{E} / \mathrm{b}_{\mathrm{a}}, \Delta \mathrm{E} / \mathrm{b}_{\mathrm{c}}<<1$

$$
\Delta \mathrm{I}=\mathrm{i}_{\mathrm{o}, \mathrm{a}} \mathrm{e}^{\wedge}\left(\mathrm{E}_{\mathrm{corr}} / \mathrm{b}_{\mathrm{a}}\right)\left(1+\Delta \mathrm{E} / \mathrm{b}_{\mathrm{a}}\right)-\mathrm{i}_{\mathrm{o}, \mathrm{c}} \mathrm{e}^{\wedge}-\left(\mathrm{E}_{\mathrm{corr}} / \mathrm{b}_{\mathrm{c}}\right)\left(1-\Delta \mathrm{E} / \mathrm{b}_{\mathrm{c}}\right)
$$


Where $\mathrm{E}_{\text {corr }} \quad$ : potential at combined anodic and cathodic current

The current and potential from this random arrangement of sites is transformed using a mathematical method like the Fast Fourier Transform, the Maximum Entropy Method, or Wavelet Analysis (which will be discussed later) into a power spectrum density like that of any non-simulated data set. The next step in the computer simulation is to change the associations between the anodic and cathodic sites. Instead of the random arrangement used initially, the sites can be arranged more regularly, figure II-4.2, or cathodic sites can be given an improved probability of occurring adjacent to an anodic site, figure II-4.3.

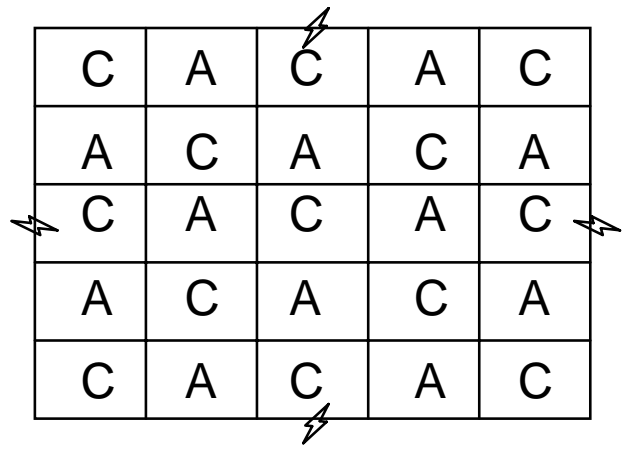

Figure II-4.2. Regular initial positions

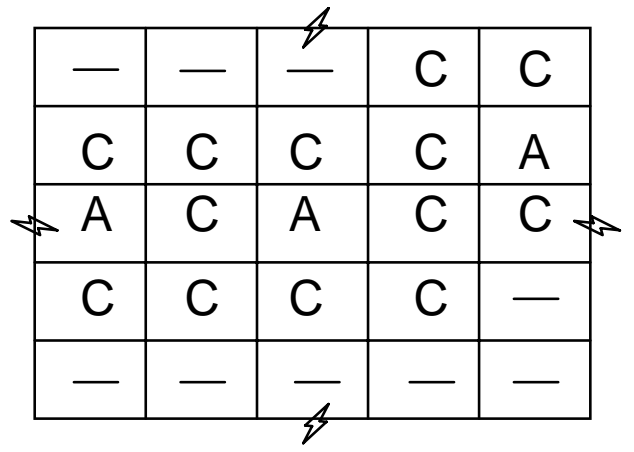

Figure II-4.3. Ordered initial positions

These both more accurately represent the possible arrangement of sites according to the PDM model [1]. In addition to changing the arrangement of sites and their probability of proximity, the likelihood of sites switching character (from anodic to cathodic, or visa versa) from moment to moment is manipulated to better represent the behavior of anodic sites transitioning from metastable to stable or inactive sites, also according to the PDM model. Figure II-4.4 illustrates a site in column 3 transitioning from anodic to a cathodic or inactive site. 


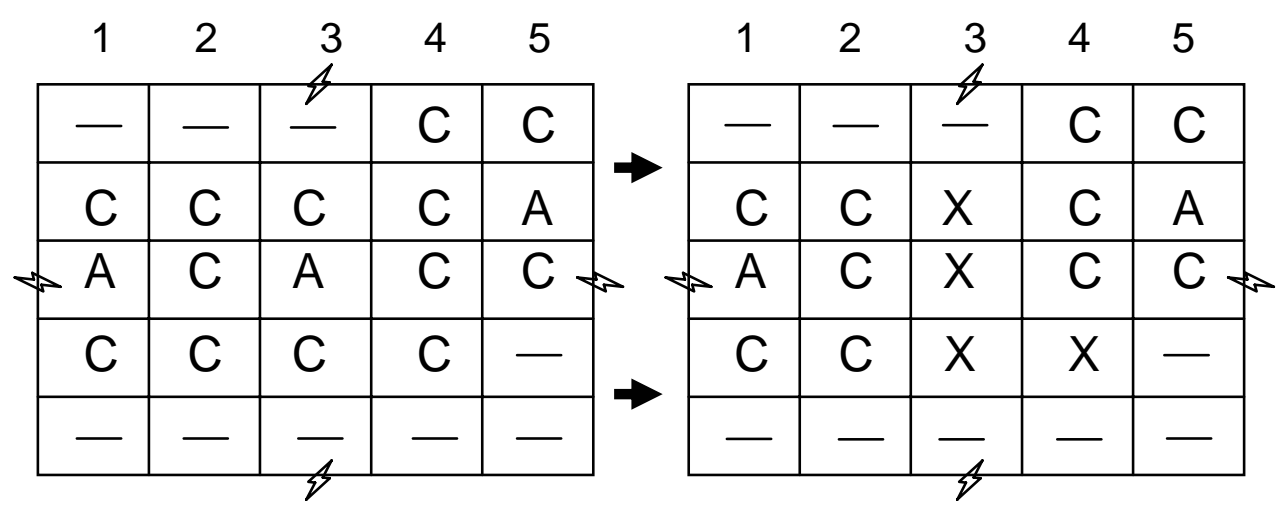

Figure II-4.4. Sites transitioning

By manipulating the rules governing the simulation, it will ultimately produce a data set and PSD plot resembling that of the natural localized corrosion processes. At this point, the rules of the simulation will provide a greater understanding of the natural process of localized corrosion, and will help refine the mathematical models of and predicting corrosion.

\section{II-4.3 Wavelet Analysis}

In the analysis of electrochemical noise data, there are a variety of methods available, some of which are mentioned above. The Fast Fourier Transformation (FFT), for example, converts data from the normal (temporal) domain into the Fourier (frequency) domain. This does two things: one, it can make it easier to compute the solution for a function that is difficult to solve in the time domain, and two, in the case of signal analysis it allows for the identification of the functions that comprise the initial signal. Fourier transformations use a combination of sine and cosine waves, superimposed on one another, to rebuild the initial signal.

The problem with FFT analysis is that it assumes a steady state. A data set which contains transients, or one in which the mean value of the data varies over time cannot be properly analyzed in FFT [2]. The signal must be normalized by subtracting out (or adding to) the changing mean to bring it to a steady state, and there is no information provided in the final analysis about the time (the point in the temporal domain) at which any of the comprising functions were contributing. Wavelet Analysis performs in a similar fashion to FFT, determining a set of "wavelets" or "basis functions" that comprise 
the original signal, but Wavelet Analysis is superior in that the basis function chosen for the analysis is limited in the time domain. This allows for the entire data set to be analyzed without losing information about an evolving signal (like electrochemical noise signals), and information is provided about the point in the time domain that a specific function was active.

Wavelet analysis works by dividing the data into two sets: one set is the "smooth" information (the baseline function), and the other set is the "detail" information. These two sets are given by wavelet coefficients; 2 for the smooth information and an integral power of 2 for the detail information (depending on how much detail is necessary) [3]. These coefficients are key to Wavelet Analysis's superiority over previous analysis methods in terms of monitoring and predicting corrosion.

Current research into corrosion and the electrochemical noise associated with each type focuses on the plateau height, roll-off slope, and roll-off frequency of the PSD plots. Research suggests that a roll-off slope of less than $20 \mathrm{~dB} /$ decade is indicative of pitting corrosion while a greater slope results from general corrosion or SCC [4]. Wavelet analysis can provide the same PSD plot that FFT or MEM can provide but it has one additional advantage. Comparing two PSD data plots like B and C, figure II-4.5, FFT and MEM cannot distinguish between the two because their features are very similar (slope and plateau height), but using Wavelet Analysis, the coefficients provided in figure II-4.6 show a clear difference between the two data sets [5].

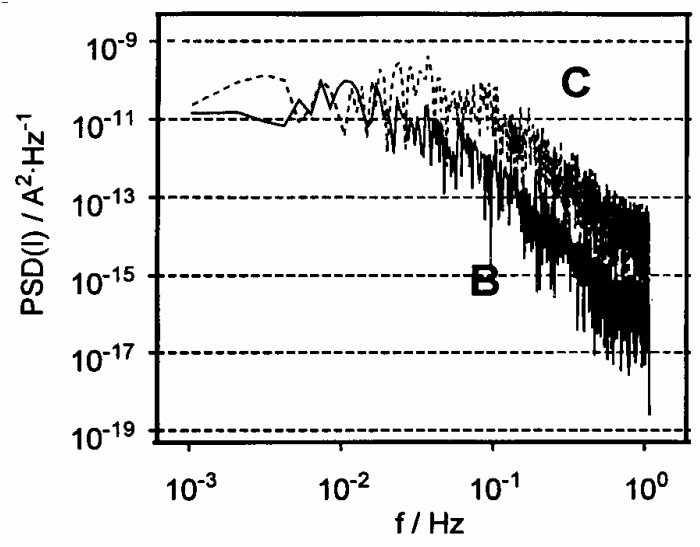

Figure II-4.5. Similar PSD plots [5] 


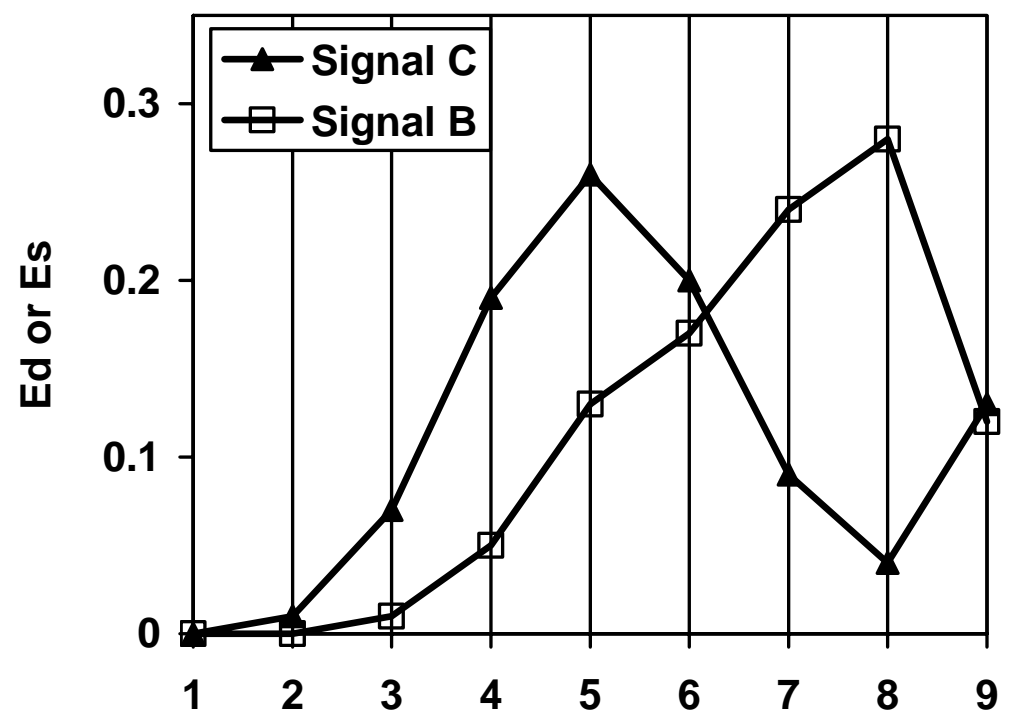

Figure II-4.6. Wavelet coefficients [5]

This quality, along with the others mentioned above, is why Wavelet Analysis will be used in the third part of this localized corrosion research. Wavelet Analysis is being performed on the general corrosion and SCC noise data from the current research, and will soon be used on the localized corrosion data as well. By comparing the PSD plots, their qualities, and the wavelet coefficients, it will be possible for the first time to definitively differentiate between the types of corrosion occurring in real-time.

\section{II-4 References}

1. D. D. Macdonald, Pure Appl. Chem., 71, 951 (1999).

2. R.A. Cottis, Corrosion, 57, 3 (2001).

3. W.H. Press, et al. Numerial Recipies in C++. Cambridge University Press, 2002. p. 599

4. Jun Liu and D.D. Macdonald, "Electrochemical Emission Spectroscopy of AISI 1018 Carbon Steel." Pennsylvania State University, 2002.

5. A. Aballe et al., Electrochimica Acta, 44, 4805 (1999). 


\section{II-5 Unification of the Deterministic and Statistical Approaches for Predicting Localized Corrosion Damage (Damage Function Analysis-DFA)}

In this section of the project, we provide an alternative, more general theoretical basis for Damage Function analysis (DFA), by drawing an analogy between the growth of a pit and the movement of a particle. In contrast to our previous formulation of DFA, which was developed specifically for enabling the damage function for localized corrosion to be calculated from the Point Defect Model for passivity breakdown, the Coupled Environment Pitting Model for pit growth, and the theory of prompt and delayed repassivation, the new formulation readily incorporates any theories or models (deterministic or empirical) for these stages in the development of a pit. We show that the new formulation leads to the original expressions for the damage functions for active (living) and passivated (dead) pits, and hence for the differential and integral damage functions, as were obtained from the original theory. We also describe the unification of deterministic (Damage Function Analysis, DFA) and empirical, statistical (Extreme Value Statistics, EVS) methods for predicting the development of localized corrosion damage on metal surfaces. In particular, we have devised a means of estimating the central and scale parameters of EVS directly from DFA in a "first principles" manner, as well as from fitting the EVS distribution function to experimental data for short times, in order to predict the extreme value distributions at longer times. The techniques have been evaluated on EVS data for the pitting of manganese steel in $\mathrm{CO}_{2}$-acidified seawater and for the pitting of aluminum in tap water. Finally, we outline the generalization of pit nucleation, as described by the Point Defect Model, for external conditions that depend on time.

It will be shown from DFA that, with certain simplifying assumptions, the distribution of the deepest pit among "identical" specimens must be described by extreme value statistics (EVS). A combination of DFA and traditional statistical analysis (SA) offers significant advantages over purely statistical/empirical approaches that are not based upon deterministic principles. Thus, DFA allows us to express the fitting parameters for SA in terms of values for the physical parameters of the component deterministic model(s) (e.g. pit nucleation and propagation rates, repassivation constants, 
etc.) that can be validated by independent experiment. Accordingly, it becomes possible to predict the statistical fitting parameters as the external conditions change with time.

\section{Research Progress}

\section{III-1 Task: Continued Development of the Theory of Passivity}

The objective of this task is to continue the development of the theory of passivity based on the Point Defect Model (PDM) [1], in order to provide a basis for interpreting experimental data on the general and localized corrosion of metals and alloys in DOE liquid waste environments. Previous work on this project was able to account for the transients in barrier layer thickness and current upon perturbing the $\mathrm{pH}$ and the potential. The current work is concentrating on the effect that temperature has on the transients. In addition we are continuing to take data at various $\mathrm{pH}$ values and potentials to ensure the accuracy of any predictions. The theory is also being extended to account for the electrochemical impedance characteristics of iron (and other passive metals) in which the dissolution of the barrier layer occurs. These models are being used to interpret impedance data for iron and other metals in borate buffer solutions under conditions where the outer layer forms or where it is prevented from forming (e.g. addition of EDTA). Optimization procedures are being used to fit the impedance models to the experimental impedance data, which are measured as functions of potential, $\mathrm{pH}$, and temperature in order to derive various model parameters as functions of the same independent variables. Our ultimate goal is to derive a single set of parameters that will account for the growth and breakdown of the passive film on iron over the entire range of conditions that are of interest in the storage of DOE liquid waste.

Eventually, the kinetic parameters will be incorporated into Damage Function Analysis which can predict passivity breakdown. Earlier work on some metals suggests that pit formation is due to the coalescence of cation vacancies at the metal/film interface. This previous work shows that once the critical vacancy density is reached, the film ruptures to form a pit. Based upon the kinetic parameters derived for the general corrosion case, two constants relating to the cation vacancy coalescence can be 
calculated. These two constants can then be applied to predict passivity breakdown. Therefore, experiments are being performed to measure pitting as a function of potential, chloride ion concentration, and temperature in high $\mathrm{pH}$ solutions, similar to that in the DOE liquid waste containers.

The immediate tasks are as follows:

1. Using EIS and ellipsometry to extract the kinetic parameters

a. Deriving an equation for the impedance of the passive film formed on iron based on the PDM

b. Obtaining impedance and thickness data for iron

c. Measuring the relative amounts of the various iron cation species present in the film via X-ray Photon Spectroscopy (XPS)

2. Using DataFit (Curve fitting software) to optimize the impedance data to the derived equation for obtaining the kinetic information

3. Obtaining data to be incorporated in DFA

\section{$\underline{\text { Task Status }}$}

\section{III-1.1a,b. Deriving the Impedance Equations from PDM}

The following part of this work is dedicated to the development of an impedance model based on the Point Defect Model (PDM) [4] in order to perform mechanistic analysis of impedance data for passive iron. Professor Macdonald provided the model and basic equations to the author.

Figure III-1.1 summarizes the physicochemical processes occurring within the passive barrier film formed on iron, in terms of the PDM. Reaction (1) describes the injection of $\mathrm{Fe}_{i}^{\chi+}$ into the barrier layer as an interstitial, and then the iron interstitial is transmitted through the barrier layer and ejected into the solution as presented by Reaction (3). Reaction (2) results in the growth of the barrier layer into the bulk metal iron, and Reaction (5) leads to the destruction of the barrier layer by dissolution. 
Reaction (4) is the consumption of an oxygen vacancy at the film/solution interface, which is produced by Reaction (2) at the metal/film interface. Note that Reactions (1), (3), and (4) are lattice conservative processes, while Reactions (2) and (5) are lattice nonconservative processes. A steady state involves two non-conservative reactions, since only one non-conservative reaction would lead to monotonic growth or thinning of the passive film.

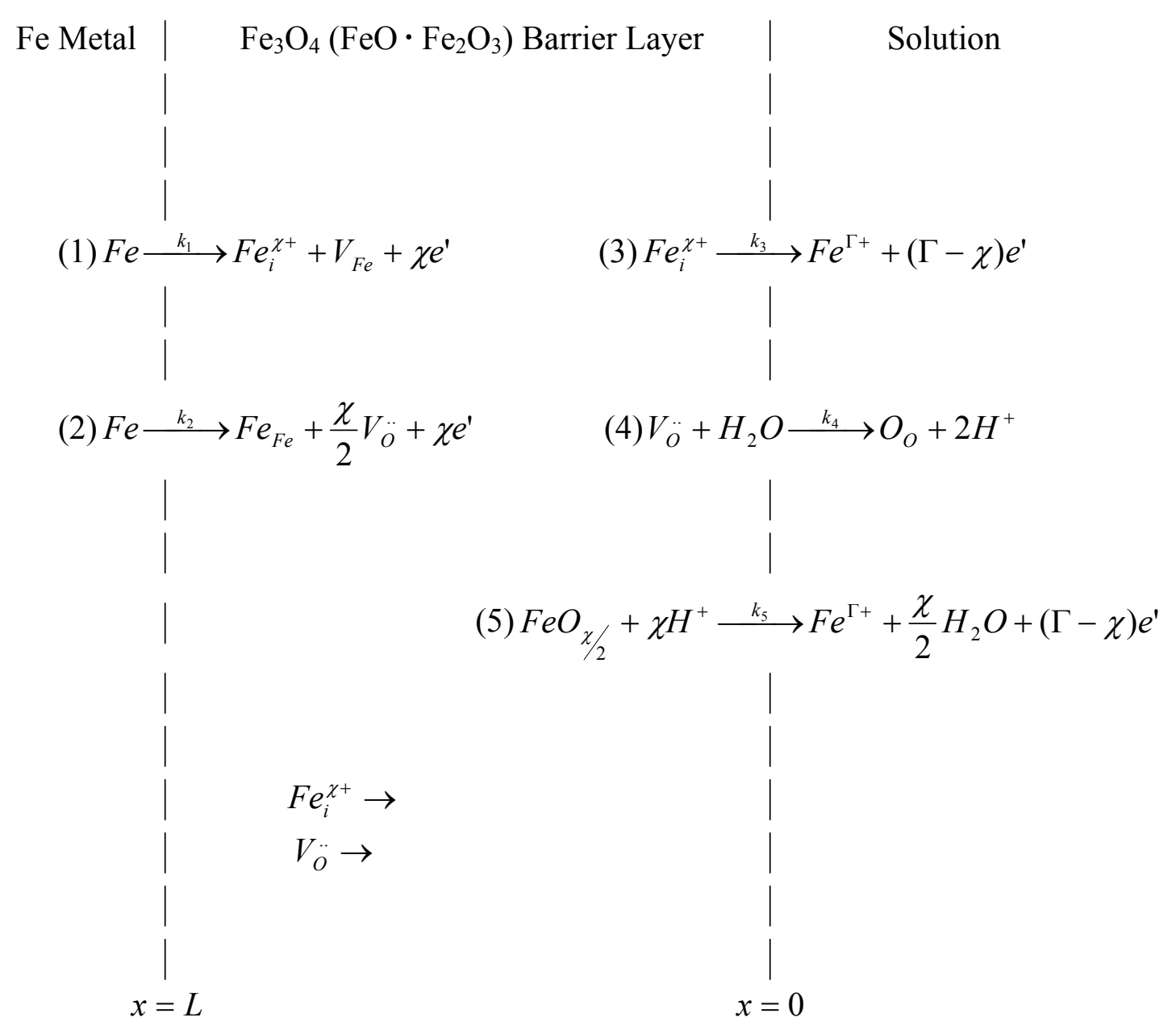

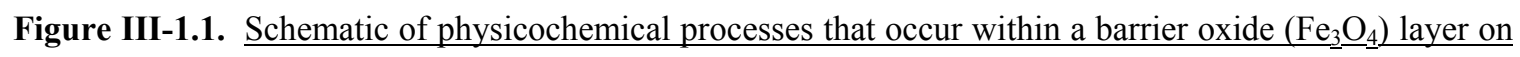
passive iron according to the Point Defect Model. $F e \equiv \underline{\text { iron atom; }} F e_{i}^{\chi+} \equiv \underline{\text { interstitial iron cation; }}$

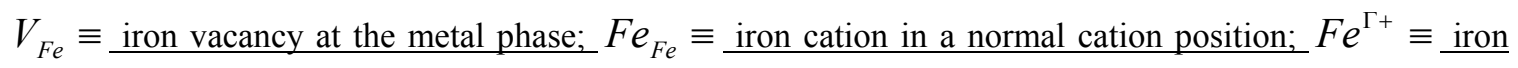
cation at the solution phase; $V_{O} \equiv$ oxygen vacancy; $O_{O} \equiv$ oxygen ion in anion site. 
Considering our previous findings that the dominant defects in the barrier layer of passive iron must be oxygen vacancies, or iron interstitials, or both, due to the n-type electronic character of the passive film, the reactions regarding the consumption (at the metal/film interface) and production (at the film/solution interface) of iron vacancies are not included in the impedance model development. Further evidence supporting this assumption is the defect formation energies for the various types of defects that can form in $\mathrm{Fe}_{2} \mathrm{O}_{3}$ (we did not find any information pertaining to magnetite). Table III-3.1 lists the formation energies and activation energies for migration for defects formed in $\mathrm{Fe}_{2} \mathrm{O}_{3}$. It can be seen that the Schottky defect (which relates to the formation of new lattice sites and includes can be either oxygen or metal vacancies) has a much lower energy of formation than the interstitials. Because oxygen interstitials are physical too large to occupy interstitial sites in the lattice, they are being ignored in our model. Table III-3.1 also lists the migration energies for the defects and they are all lower than the formation energies, which means that if defects can form, they can easily diffuse.

Table III-3.1 Table listing the defect formation energies and activation energies for migration for defects formed in $\mathrm{Fe}_{2} \mathrm{O}_{3}[]$.

\begin{tabular}{|c|c|c|c|}
\hline Defect & Formation Energy (eV) & Mobile Species & $\begin{array}{c}\text { Activation Energies } \\
(\mathbf{e V})\end{array}$ \\
\hline Cation Interstitial & 6.05 & Cation Vacancy & 2.14 \\
\hline Anion Interstitial & 5.45 & Anion Vacancy & 2.24 \\
\hline Schottky (Vo or Vm) & 4.42 & Cation Interstitial & 2.08 \\
\hline
\end{tabular}

The change in film thickness with time involves two lattice non-conservative reactions, consequently determined as follows:

$$
\frac{d L}{d t}=-\frac{2 \Omega}{\chi} J_{o}-\Omega k_{5} C_{H^{+}}^{n}
$$


in which $\Omega=14.9 \mathrm{~cm}^{3} \mathrm{~mol}^{-1}$ is the volume per mole of the barrier layer, $\chi=8 / 3$ is the oxidation state of iron in the barrier layer, $J_{o}$ is the flux for oxygen vacancies within the barrier layer, $C_{H^{+}}$is the hydrogen ion concentration in the solution at the film/solution interface, $n$ is the kinetic order of the film dissolution reaction with respect to $C_{H^{+}}$at the film/solution interface, and $k_{5}$ is the rate constant for Reaction (5). Table III-1.2 displays rate constants for five interfacial reactions; the definition of standard rate constants will be detailed later in this section.

All electron-related interfacial reactions contribute to the total current density, which is described as

$I=F\left[-\chi J_{i}^{m / f}-2 J_{o}^{m / f}-(\Gamma-\chi) J_{i}^{f / s}+(\Gamma-\chi) k_{5} C_{H^{+}}^{n}\right]$

in which $F=96487 \mathrm{C} / \mathrm{mol}$ is Faraday's constant, and $\Gamma=3$ is the oxidation state of iron in solution. The fluxes are written as $J_{i}=-D_{i} \frac{\partial C_{i}}{\partial x}-\chi K D_{i} C_{i}$ for iron interstitials and $J_{o}=-D_{o} \frac{\partial C_{o}}{\partial x}-2 K D_{o} C_{o}$ for oxygen vacancies, where $D$ and $C$ are diffusivity and concentration, subscripts $i$ and $o$ are for iron interstitials and oxygen vacancies, $K=\varepsilon F / R T, R=8.314 \mathrm{Jmol}^{-1} K^{-1}$ is the gas constant, $T$ is the absolute temperature, and $\varepsilon$ is the electric field strength, which is considered a constant according to the PDM. The continuity equations $\frac{\partial C}{\partial t}=-\nabla J$ then become

$$
\begin{aligned}
& \frac{\partial C_{i}}{\partial t}=D_{i} \frac{\partial^{2} C_{i}}{\partial x^{2}}+\chi D_{i} K \frac{\partial C_{i}}{\partial x} \\
& \frac{\partial C_{o}}{\partial t}=D_{i} \frac{\partial^{2} C_{o}}{\partial x^{2}}+2 D_{o} K \frac{\partial C_{o}}{\partial x}
\end{aligned}
$$


the initial conditions $(\mathrm{t}=0)$ of which are $C_{i}(x)=C_{i}^{0}(x)$ and $C_{o}(x)=C_{o}^{0}(x)$ and the boundary conditions of which are: at $x=0$ (the film/solution interface): $J_{i}(0)=-k_{3}^{0} e^{a_{3} V} C_{i}(0)\left(k_{3}^{0}\right.$ is in $\left.\mathrm{cm} / \mathrm{s}\right) ; J_{o}(0)=-\left(\frac{\chi}{2}\right) k_{4}^{0} e^{a_{4} V} C_{o}(0)\left(k_{4}^{0}\right.$ is in $\left.\mathrm{cm} / \mathrm{s}\right)$; at $x=L \quad$ (the metal/film interface): $J_{i}(L)=-k_{1}^{0} e^{a_{1} V} e^{-b_{1} L} \quad\left(k_{1}^{0} \quad\right.$ is in $\left.\mathrm{mol} / \mathrm{s} / \mathrm{cm}^{2}\right)$; $J_{o}(L)=-\left(\frac{\chi}{2}\right) k_{2}^{0} e^{a_{2} V} e^{-b_{2} L}\left(k_{2}^{0}\right.$ is in $\left.\mathrm{mol} / \mathrm{s} / \mathrm{cm}^{2}\right)$.

Combined with the rate constants shown in Table III-1.2, the rate of film thickness change in Equation (1) and current density in Equation (2) then become

$$
\begin{gathered}
\frac{d L}{d t}=-\Omega k_{2}^{0} e^{a_{2} V} e^{-b_{2} L}-\Omega k_{5}^{0} e^{a_{5} V} C_{H^{+}}^{n} \\
I=F\left[\chi k_{1}^{0} e^{a_{1} V} e^{-b_{1} L}+\chi k_{2}^{0} e^{a_{2} V} e^{-b_{2} L}+(\Gamma-\chi) k_{3}^{0} e^{a_{3} V} C_{i}(0)+(\Gamma-\chi) k_{5}^{0} e^{a_{5} V} C_{H^{+}}^{n}\right]
\end{gathered}
$$

Note that the current density is a function of $V, L, C_{i}(0)$. Accordingly, for any arbitrary changes $\delta V, \delta L, \delta C_{i}(0)$

$\delta I=\left(\frac{\partial I}{\partial V}\right)_{L, C_{i}(0)} \delta V+\left(\frac{\partial I}{\partial L}\right)_{V, C_{i}(0)} \delta L+\left(\frac{\partial I}{\partial C_{i}(0)}\right)_{V, L} \delta C_{i}(0)$

in which the variations are sinusoidal in terms of EIS, i.e., $\delta V=\Delta V e^{j \omega t} ; \delta L=\Delta L e^{j \omega t}$; $\delta C_{i}(0)=\Delta C_{i}(0) e^{j \omega t}$, note that $\Delta x$ is the amplitude of the variation in $X$ at a frequency $\omega=0$. 
Table III-1.2. Rate constants $k_{i}=k_{i}^{0} e^{a_{i} V} e^{-b_{i} L}$ for five interfacial reactions in terms of the Point Defect Model.

\begin{tabular}{|c|c|c|c|}
\hline Reaction & $\begin{array}{l}a_{i} \\
\left(V^{-1}\right)\end{array}$ & $\begin{array}{l}b_{i} \\
\left(\mathrm{~cm}^{-1}\right)\end{array}$ & Unit of $k_{i}^{0}$ \\
\hline $\begin{array}{l}\text { (1) } \\
\mathrm{Fe} \stackrel{k_{1}}{\longrightarrow} \mathrm{Fe}_{i}^{\chi+}+V_{\mathrm{Fe}}+\chi e^{\prime}\end{array}$ & $\alpha_{1}(1-\alpha) \chi \gamma$ & $\alpha_{1} \chi K$ & $\frac{\mathrm{mol}}{\mathrm{cm}^{2} \mathrm{~s}}$ \\
\hline $\begin{array}{l}\text { (2) } \\
\mathrm{Fe} \stackrel{k_{2}}{\longrightarrow} \mathrm{Fe}_{\mathrm{Fe}}+\frac{\chi}{2} V_{\ddot{o}}+\chi e^{\prime}\end{array}$ & $\alpha_{2}(1-\alpha) \chi \gamma$ & $\alpha_{2} \chi K$ & $\frac{\mathrm{mol}}{\mathrm{cm}^{2} \mathrm{~s}}$ \\
\hline $\begin{array}{l}\text { (3) } \\
F e_{i}^{\chi+} \stackrel{k_{3}}{\longrightarrow} F e^{\Gamma+}+(\Gamma-\chi) e^{\prime}\end{array}$ & $\alpha_{3} \alpha \Gamma \gamma$ & 0 & $\frac{\mathrm{cm}}{\mathrm{s}}$ \\
\hline $\begin{array}{l}\text { (4) } \\
V_{O}+H_{2} O \stackrel{k_{4}}{\longrightarrow} O_{O}+2 H^{+}\end{array}$ & $2 \alpha_{4} \alpha \gamma$ & 0 & $\frac{\mathrm{cm}}{\mathrm{s}}$ \\
\hline $\mathrm{FeO}_{\chi / 2}+\chi \mathrm{H}^{+} \stackrel{k_{5}}{\longrightarrow} \mathrm{Fe}^{\Gamma+}+\frac{\chi}{2} \mathrm{H}_{2} \mathrm{O}+(\Gamma-\chi) e^{\prime}$ & $\alpha_{5} \alpha(\Gamma-\chi) \gamma$ & 0 & $\frac{\mathrm{mol}^{0.4}}{\mathrm{~cm}^{0.2} \mathrm{~s}}$ \\
\hline
\end{tabular}

From Equation (7), the Faradic admittance is defined as

$$
Y_{f}=\frac{\delta I}{\delta V}=I^{V}+I^{L} \frac{\Delta L}{\Delta V}+I^{C_{i}(0)} \frac{\Delta C_{i}(0)}{\Delta V}
$$

More simply stated, $I^{V}=\left(\frac{\partial I}{\partial V}\right)_{L, C_{i}(0)} ; I^{L}=\left(\frac{\partial I}{\partial L}\right)_{V, C_{i}(0)} ; I^{C_{i}(0)}=\left(\frac{\partial I}{\partial C_{i}(0)}\right)_{L, V} . \quad$ These differentials will be evaluated later from Equation (6). 
Now we return to Equation (5) and determine the response of $\frac{d L}{d t}$ to $\delta V, \delta L$, and $\delta C_{i}(0)$ by taking the total differential. Thus, for the relaxation in film thickness: $\frac{d}{d t}(\delta L)=\Omega k_{2}^{0} a_{2} e^{a_{2} V} e^{-b_{2} L} \delta V-\Omega k_{2}^{0} b_{2} e^{a_{2} V} e^{-b_{2} L} \delta L-\Omega k_{5}^{0} a_{5} e^{a_{5} V} C_{H^{+}}^{n} \delta V . \quad$ Considering that $\frac{d}{d t}(\delta L)=j \omega \Delta L e^{j w t}$, so that

$j \omega \Delta L e^{j \omega t}=\Omega\left(k_{2}^{0} a_{2} e^{a_{2} V} e^{-b_{2} L}-k_{5}^{0} a_{5} e^{a_{5} V} C_{H^{+}}^{n}\right) \Delta V e^{j \omega t}-\Omega k_{2}^{0} b_{2} e^{a_{2} V} e^{-b_{2} L} \Delta L e^{j \omega t}$, and finally

$\frac{\Delta L}{\Delta V}=\frac{\Omega\left(k_{2}^{0} a_{2} e^{a_{2} V} e^{-b_{2} L}-k_{5}^{0} a_{5} e^{a_{5} V} C_{H^{+}}^{n}\right)}{\Omega k_{2}^{0} b_{2} e^{a_{2} V} e^{-b_{2} L}+j \omega}$ or $\frac{\Delta L}{\Delta V}=\frac{\Phi_{2}}{1+j \omega \tau_{2}}$

where $\quad \Phi_{2}=\left(\frac{a_{2}}{b_{2}}\right)-\left(\frac{k_{5}^{0}}{k_{2}^{0}}\right)\left(\frac{a_{5}}{b_{2}}\right) e^{\left(a_{5}-a_{2}\right) V} e^{b_{2} L} C_{H^{+}}^{n}$

$$
\tau_{2}=\frac{1}{\Omega k_{2}^{0} b_{2} e^{a_{2} V} e^{-b_{2} L}}
$$

For iron interstitials, the relaxation for $C_{i}(0)$ is determined through the mass balance at the film/solution interface $\frac{d C_{i}(0)}{d t}=-k_{3}^{\prime} C_{i}(0)=-k_{3}^{\prime 0} e^{a_{3} V} C_{i}(0)\left(\right.$ Unit of $k_{3}^{\prime 0}$ is s $\left.{ }^{-1}\right)$. The total differential is $\frac{d}{d t}\left(\delta C_{i}(0)\right)=-k_{3}^{\prime 0}\left[a_{3} e^{a_{3} V} C_{i}(0) \delta V+e^{a_{3} V} \delta C_{i}(0)\right]$. Substituting in the sinusoidal variations for $\delta V$ and $\delta C_{i}(0)$, one obtains $j \omega \Delta C_{i}(0) e^{j \omega t}=-k_{3}^{\prime 0} a_{3} e^{a_{3} V} C_{i}(0) \Delta V e^{j \omega t}-k_{3}^{\prime 0} e^{a_{3} V} \Delta C_{i}(0) e^{j \omega t}$ and $\Delta C_{i}(0)\left(j \omega+k_{3}^{\prime 0} e^{a_{3} V}\right)=-k_{3}^{\prime 0} a_{3} e^{a_{3} V} C_{i}(0) \Delta V$. Therefore, 


$$
\frac{\Delta C_{i}(0)}{\Delta V}=\frac{-k_{3}^{\prime 0} a_{3} e^{a_{3} V} C_{i}(0)}{j \omega+k_{3}^{\prime 0} e^{a_{3} V}}=-\frac{a_{3} C_{i}(0)}{1+j \omega \tau_{3}}
$$

in which $\quad \tau_{3}=\frac{1}{k_{3}^{\prime 0} e^{a_{3} V}}$

The expression for the faradic admittance is finally derived from Equation (8) as

$$
Y_{f}=I^{V}+I^{L} \frac{\Phi_{2}}{1+j \omega \tau_{2}}-I^{C_{i}(0)} \frac{a_{3} C_{i}(0)}{1+j \omega \tau_{3}}+j \omega C
$$

in which the parallel geometric capacitance $C=\frac{\hat{\varepsilon} \varepsilon_{0}}{L}$ (dielectric constant $\hat{\varepsilon}=30$ for passive iron [12] and vacuum permittivity $\varepsilon_{0}=8.85 \times 10^{-14} \mathrm{~F} / \mathrm{cm}$ ) is taken into consideration, and

$$
\begin{aligned}
& I^{V}=\left(\frac{\partial I}{\partial V}\right)_{L, C_{i}(0)} \\
& =F\left[a_{1} \chi k_{1}^{0} e^{a_{1} V} e^{-b_{1} L}+a_{2} \chi k_{2}^{0} e^{a_{2} V} e^{-b_{2} L}+a_{3}(\Gamma-\chi) k_{3}^{0} e^{a_{3} V} C_{i}(0)+a_{5}(\Gamma-\chi) k_{5}^{0} e^{a_{5} V} C_{H^{+}}^{n}\right] \\
& I^{L}=\left(\frac{\partial I}{\partial L}\right)_{V, C_{i}(0)} \\
& =F\left[-b_{1} \chi k_{1}^{0} e^{a_{1} V} e^{-b_{1} L}-b_{2} \chi k_{2}^{0} e^{a_{2} V} e^{-b_{2} L}\right]=-\chi F\left[b_{1} k_{1}^{0} e^{a_{1} V} e^{-b_{1} L}+b_{2} k_{2}^{0} e^{a_{2} V} e^{-b_{2} L}\right] \\
& I^{C_{i}(0)}=\left(\frac{\partial I}{\partial C_{i}(0)}\right)_{V, L}=(\Gamma-\chi) F k_{3}^{0} e^{a_{3} V}
\end{aligned}
$$


In the above expressions, the parameters that appear on the right side $\left(L\right.$ and $\left.C_{i}(0)\right)$ are identified with the steady-state quantities. At steady state, Equation (3) and (4) turn into

$$
\begin{aligned}
& \frac{\partial^{2} C_{i}}{\partial x^{2}}+\chi K \frac{\partial C_{i}}{\partial x}=0 \\
& \frac{\partial^{2} C_{o}}{\partial x^{2}}+2 K \frac{\partial C_{o}}{\partial x}=0
\end{aligned}
$$

the solutions to which are

$$
\begin{aligned}
& C_{i}(x)=A_{i} e^{-\chi K x}+B_{i} \\
& C_{o}(x)=A_{o} e^{-2 K x}+B_{o}
\end{aligned}
$$

The expressions for the fluxes therefore become

$$
\begin{aligned}
& J_{i}=-\chi K D_{i} B_{i} \\
& J_{o}=-2 K D_{o} B_{o}
\end{aligned}
$$

From the boundary conditions, the coefficients are obtained as

$$
B_{i}=\frac{k_{1}^{0} e^{a_{1} V} e^{-b_{1} L}}{\chi K D_{i}}
$$




$$
B_{o}=\frac{\left(\frac{\chi}{2}\right) k_{2}^{0} e^{a_{2} V} e^{-b_{2} L}}{2 K D_{o}}
$$

The fluxes for a given species at two interfaces are equal at steady state:

$-k_{3}^{0} e^{a_{3} V} C_{i}(0)=-k_{1}^{0} e^{a_{1} V} e^{-b_{1} L}$ and $-\left(\frac{\chi}{2}\right) k_{4}^{0} e^{a_{4} V} C_{o}(0)=-\left(\frac{\chi}{2}\right) k_{2}^{0} e^{a_{2} V} e^{-b_{2} L}$, the steady-state concentrations for iron interstitials and oxygen vacancies at the film/solution interface are

$$
\begin{aligned}
& C_{i}(0)=\left(\frac{k_{1}^{0}}{k_{3}^{0}}\right) e^{\left(a_{1}-a_{3}\right) V} e^{-b_{1} L} \\
& C_{o}(0)=\left(\frac{k_{2}^{0}}{k_{4}^{0}}\right) e^{\left(a_{2}-a_{4}\right) V} e^{-b_{2} L}
\end{aligned}
$$

In the case of steady state, Equation (1) becomes

$$
J_{o}(L)=-\left(\frac{\chi}{2}\right) k_{5} C_{H^{+}}^{n}
$$

and from Equations (23) and (25): $-\left(\frac{\chi}{2}\right) k_{5} C_{H^{+}}^{n}=-2 K D_{o}\left(\frac{\left(\frac{\chi}{2}\right) k_{2}^{0} e^{a_{2} V} e^{-b_{2} L}}{2 K D_{0}}\right)$ Therefore, the steady-state barrier layer thickness is 


$$
L=\left(\frac{a_{2}-a_{5}}{b_{2}}\right) V-\frac{1}{b_{2}} \ln \left[\left(\frac{k_{5}^{0}}{k_{2}^{0}}\right) C_{H^{+}}^{n}\right]
$$

The parameters, $k_{1}^{0}, k_{2}^{0}, k_{3}^{0}, k_{3}^{\prime 0}, k_{4}^{0}, k_{5}^{0}$, in the above equations and Table III1.1 are a function of solution $\mathrm{pH}$ and are not the standard rate constants for interfacial reactions, since the PDM defines $k_{1}^{0}=k_{1}^{00} e^{-\alpha_{1} \beta \chi \gamma p H} e^{-\alpha_{1} \chi \gamma \phi_{f / s}^{0}}, k_{2}^{0}=k_{2}^{00} e^{-\alpha_{2} \beta \chi \gamma p H} e^{-\alpha_{2} \chi \gamma \phi_{f / s}^{0}}$, $k_{3}^{0}=k_{3}^{00} e^{\alpha_{3} \beta \Gamma \gamma p H} e^{\alpha_{3} \Gamma \gamma \phi_{f / s}^{0}}, \quad k_{3}^{\prime 0}=k_{3}^{00} e^{\alpha_{3} \beta \Gamma \gamma p H} e^{\alpha_{3} \Gamma \gamma \phi_{f / s}^{0}}, \quad k_{4}^{0}=k_{4}^{00} e^{2 \alpha_{4} \beta \gamma p H} e^{2 \alpha_{4} \gamma \phi_{f / s}^{0}}, \quad$ and $k_{5}^{0}=k_{5}^{00} e^{\alpha_{5} \beta(\Gamma-\chi) \gamma p H} e^{\alpha_{5}(\Gamma-\chi) \gamma \phi_{f / s}^{0}}$, in which $\alpha_{1}, \alpha_{2}, \alpha_{3}, \alpha_{4}, \alpha_{5}$ are the transfer coefficients, and $k_{1}^{00}, k_{2}^{00}, k_{3}^{00}, k_{3}^{00}, k_{4}^{00}, k_{5}^{00}$ are the standard rate constants. The potential drop across the film/solution interface is $\phi_{f / s}=\phi_{f}-\phi_{s}=\alpha V+\beta p H+\phi_{f / s}^{0}$ and that across the metal/film interface is $\phi_{m / f}=\phi_{m}-\phi_{s}=(1-\alpha) V-\varepsilon L-\beta p H-\phi_{f / s}^{0}$, in which $\alpha=\frac{d \phi_{f / s}}{d V}$ is the dependence of the potential drop across the film/solution interface on $V$, $\beta=\frac{d \phi_{f / s}}{d p H}$ is the dependence of the potential drop across the film/solution interface on $p H, \quad \phi_{f / s}^{0}$ is the value of $\phi_{f / s}$ at standard state; $\gamma=\frac{F}{R T}, \quad K=\varepsilon \gamma=\frac{\varepsilon F}{R T}$, $\varepsilon=1.10 \times 10^{6} \mathrm{~V} / \mathrm{cm}$, and $\alpha=0.728$ for passive iron [3].

Typical experimental impedance spectra for passive iron are shown in Figure III-1.2, in which real and imaginary parts of the impedance are plotted as a function of the measurement frequency with 20 data points evenly spaced in each frequency decade. In the high frequency range, real and imaginary parts of the impedance are almost independent of the measurement frequency and exhibit very small absolute values. DataFit software (version 7.1) was employed in this work in order to obtain the transfer coefficient $\alpha_{i}$ and standard rate constant $k_{i}^{00}$ for the $i$-th elementary interfacial reaction via nonlinear regression of experimental data, based on the derivation of an impedance model from the PDM. The values of $\beta$ and $\phi_{f / s}^{0}$ were also generated for passive iron through this method. The optimization algorithm depends on finding the minimum 
residual sum of squares between experimental data and simulated data. Details regarding the nonlinear regression procedure are described in the next section for a typical analysis of impedance data. Fundamental parameters for passive iron are presented in Table III1.2 and a comparison between experimental data and simulated data is displayed in Figure III-1.3.

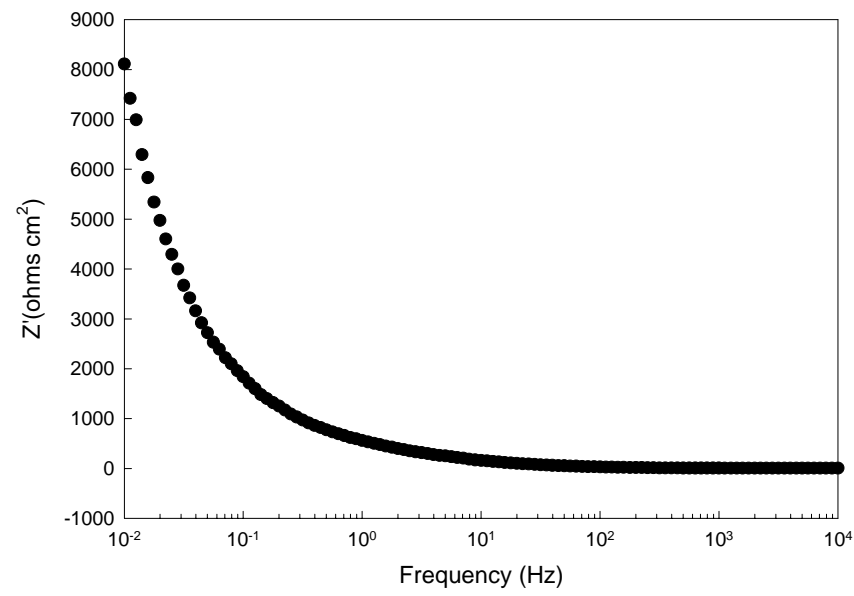

(a)

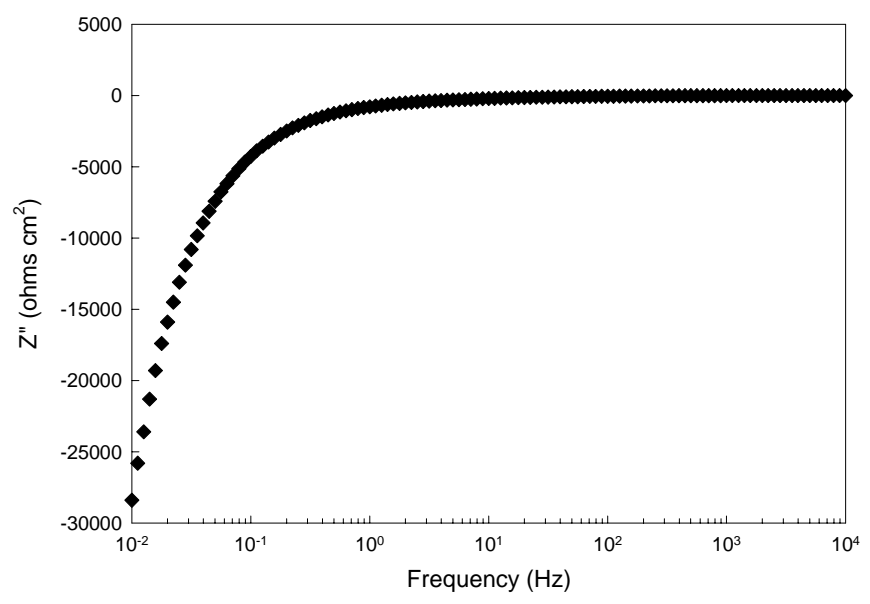

(b)

Figure III-1.2. Real part (a) and imaginary part (b) of impedance data for the passive film formed on iron in borate buffer solution with $0.01 \mathrm{M}$ EDTA $(\mathrm{pH} \mathrm{8.15)}$ at an applied film formation voltage of $0.2 \mathrm{~V}$ vs. SCE, plotted as a function of experimental frequencies ranging from $10^{-2} \mathrm{~Hz}$ to $10^{4} \mathrm{~Hz}$. 
Table III-1.3. Fundamental parameters for the passive film formed on iron.

\begin{tabular}{|l|l|l|}
\hline Parameter & Room Temp. & $\mathbf{7 5 C}$ \\
\hline$\alpha_{1}$ & 0.01 (from regression) & 0.0007 (from regression) \\
\hline$\alpha_{2}$ & 0.24 (from regression) & 0.06 (from regression) \\
\hline$\alpha_{3}$ & 0.39 (from regression) & 0.45 (from regression) \\
\hline$\alpha_{5}$ & 0.30 (from regression) & 0.12 (from regression) \\
\hline$k_{1}^{00}\left(\mathrm{~mol} \cdot \mathrm{cm}^{-2} \cdot \mathrm{s}^{-1}\right)$ & $3.8 \times 10^{-12}$ (from regression) & $9.9 \times 10^{-10}$ (from regression) \\
\hline$k_{2}^{00}\left(\mathrm{~mol} \cdot \mathrm{cm}^{-2} \cdot \mathrm{s}^{-1}\right)$ & $1.1 \times 10^{-15}$ (from regression) & $1.8 \times 10^{-20}$ (from regression) \\
\hline$k_{3}^{00}\left(\mathrm{~s}^{-1}\right)$ & $2.4 \times 10^{-6}$ (from regression) & $3.85 \times 10^{-7}$ (from regression) \\
\hline$k_{5}^{00}\left(\mathrm{~mol} \mathrm{l}^{0.4} \cdot \mathrm{cm}^{-0.2} \cdot \mathrm{s}^{-1}\right)$ & $3.3 \times 10^{-8}$ (from regression) & $4.61 \times 10^{-8}$ (from regression) \\
\hline$\beta$ & -0.0047 (from regression) & .0002 (from regression) \\
\hline$\phi_{f / s}^{0}(\mathrm{Vvs} . \mathrm{SCE})$ & -0.29 (from regression) & -.007 (from regression) \\
\hline$\hat{\varepsilon}$ & 30 (from Reference [3]) & 30 (from Reference [3]) \\
\hline$\varepsilon(\mathrm{V} / \mathrm{cm})$ & $1.10 \times 10^{6} \quad$ (from Reference & $1.10 \times 10^{6} \quad$ (from Reference \\
$[12])$ & $[12])$ \\
\hline$\alpha$ & 0.728 (from Reference [12]) & 0.728 (from Reference [12]) \\
\hline
\end{tabular}




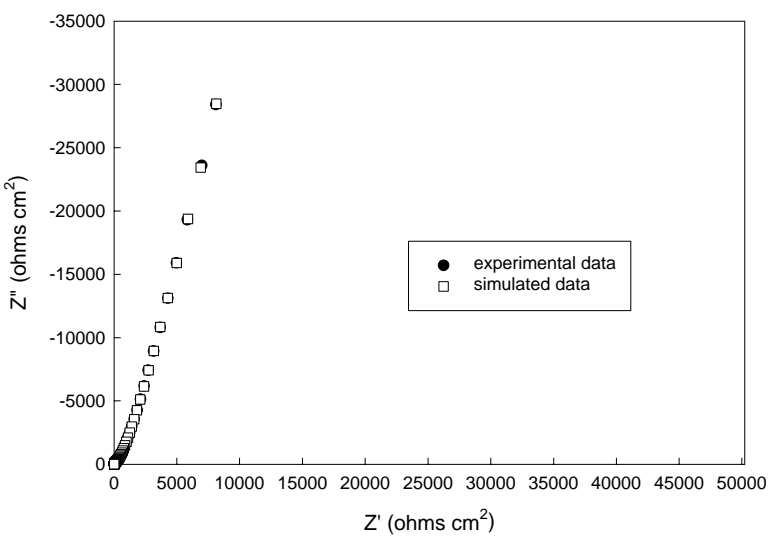

(a)

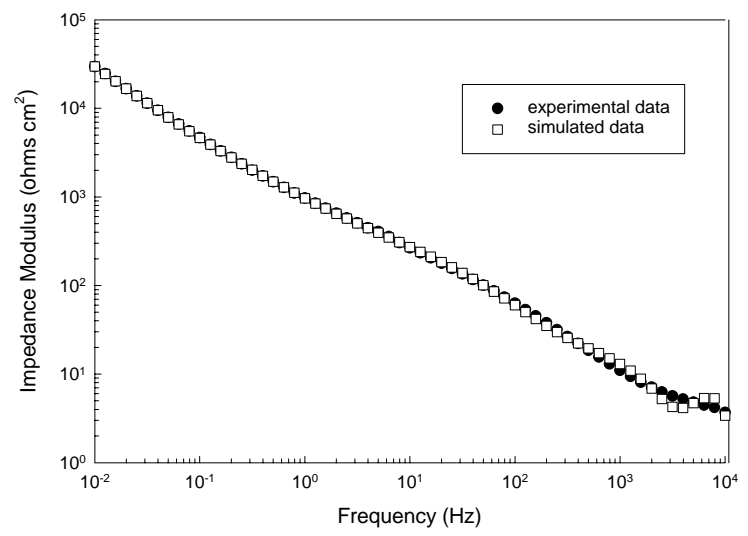

(b)

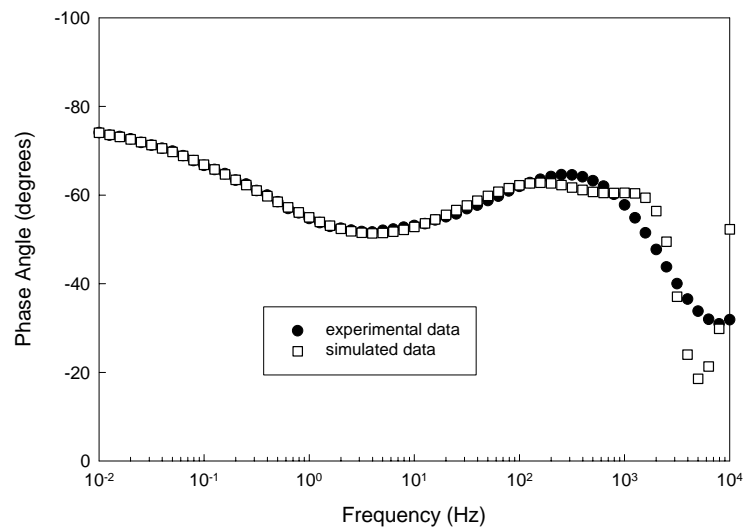

(c)

Figure III-1.3. Nyquist plot (a) and Bode plots (b, c) of impedance data for the passive film formed on iron in borate buffer solution with $0.01 \mathrm{M}$ EDTA $(\mathrm{pH} \mathrm{8.15)}$ at an applied film formation voltage of $0.2 \mathrm{~V}$ vs. SCE. Closed circles represent experimental data and open squares represent simulated data using nonlinear fitted parameters. 
The correlation between experimental impedance data and simulated data is good for Nyquist plots and modulus Bode plots in most cases. This validates the impedance model based on the PDM, through which a single set of parameters for passive iron (shown in Table III-1.3) have been derived via nonlinear regression in order to obtain simulated impedance data. However, the phase angle Bode plot displays some difference between experimental data and simulated data, especially in higher frequency range. A possible reason is that the phase angle, defined in Equation (7) as $\theta=\tan ^{-1} Z^{\prime \prime} / Z^{\prime}$, is a very sensitive function of $Z^{\prime}$ and $Z^{\prime \prime}$, so that a slight deviation in $Z^{\prime}$ or $Z^{\prime \prime}$ could give rise to a large fluctuation in $\theta$, which has a value only between $0^{\circ}$ and $-90^{\circ}$ for valid electrochemical impedance data.

Table III-1.3 shows that the standard rate constant for the iron interstitial production reaction (Reaction (1) in Figure III-1.1) is higher than that of the oxygen vacancy production reaction (Reaction (2) in Figure III-1.1) by several orders of magnitude. This implies that the generation of iron interstitials at the metal/film interface is much easier, considering that Reaction (1) is a lattice conservative process while Reaction (2) is not. However, the transfer coefficient $\alpha_{1}$ of Reaction (1) is close to 0 , which indicates that the intermediate activated complex for the charge transfer reaction (Reaction (1)) is very similar to the reactant $\mathrm{Fe}$, rather than the product $\mathrm{Fe}_{i}^{\chi+}$. Such observations are in agreement with the findings that that the dominant defects in the passive film formed on iron are oxygen vacancies, or iron interstitials, or both, due to the $\mathrm{n}$-type electronic properties. The value of $\beta$ is found to be -0.0047 , which is very close to that found in previous work [3], suggesting that $\beta$ has a value of 0 for the passive film formed on iron in borate buffer solutions.

Some regression analysis has been performed on the data taken at $75^{\circ} \mathrm{C}$. The results are shown in the third column of Table III-1.3. The results represent the fit for one set of data, however, fits will be made to two more sets of data to ensure that the parameters are in good agreement. It should be noted that the reaction rate constant for the injection of $\mathrm{Fe}_{i}^{\chi^{+}}$into the film is greatly increased (compared to room temp.), while 
the other reaction rate constants remain approximately the same or even decrease slightly. This was found to be the trend for all of the measured potentials.

It is well accepted that reaction rates are highly dependent on the temperature due to the relationship with the activation energy for a reaction. Despite this fact, it appears as if only one of the reaction rates increased by a substantial amount. This finding helps to explain the mechanism responsible for the higher steady state current observed at $75^{\circ}$ $\mathrm{C}$, which is $2.1 \pm 0.1 \mu \mathrm{A} / \mathrm{cm}^{2}$ (two orders of magnitude larger than that at room temp.). The information obtained here suggests that at higher temperatures, $\mathrm{Fe}_{i}^{\chi+}$ is being injected into the film much more rapidly and diffuses through the oxide layer much faster to account for the high steady state currents. These are the early findings on the temperature effects on the kinetics. Further analysis will be carried out to acquire more precise kinetic parameters. In addition a Warburg impedance (due to diffusion of defects in the film) will be incorporated in the model to determine if defect diffusion plays a role in the overall impedance of the film.

The parameters in Table III-1.3 were used to calculate the steady-state properties for room temperature, including film thickness and current density, of passive films formed on iron. The results are shown in Figure III-1.4 and good agreement has been achieved between simulated data and experimental data from our previous research. This becomes another evidence for the validity of the PDM. Figure III-1.5 compares the thickness measured by ellipsometry to the thickness calculated through the assumption that the oxide film behaves as a parallel plate capacitor. It should be noted that a slight difference in thickness between figure III-1.4 and figure III-1.5. Figure III-1.4 displays the data obtained by earlier workers on this project, while figure III-1.5 shows more recent data. Although the thickness differs slightly, the slopes are nearly identical for room temperature. In addition, figure III-1.5 shows the thickness values as a function of formation voltage for films grown at $75^{\circ} \mathrm{C}$ with all other conditions being constant. Further regression will be carried out on the data to obtain the parameters and comparisons of the simulated data to the experimental data will be made. This analysis 
will be performed for $3 \mathrm{pH}$ values, 3 temperatures, and 3 formation voltages to ensure that good agreement is made between the experimental and simulated data.

\section{Issues and Concerns:}

The equations derived from PDM to describe the film under steady-state conditions do not account for the diffusivities of the various species in the film. The approach used to derive these equations clearly shows that the diffusion coefficients "drop out" when the equations are derived using the boundary conditions under steady state conditions. While there is no evidence of a mistake in this approach, we would intuitively expect that the diffusivities should play a role in the film's impedance. For this reason, we are currently incorporating a Warburg impedance (due to defect diffusion) to account for any impedance caused by this process.

In addition to our work on the modeling, we also used ellipsometry to support the claim adding EDTA solution will remove the outer precipitation layer. Table III-1.6 shows the results of that experiment. It should be noted that a regression fit to the measured ellipsometry parameters $(\operatorname{Tan}(\Psi)$ and $\operatorname{Cos}(\Delta))$ was used in both cases. For the case when EDTA was not added to the solution, no fit could be obtained. However, a two layer model provided an excellent fit to the data (not shown in this report). Looking at Table III-1.6, the effects of EDTA are quite evident. First, the results show that only a one layer film formed in solutions containing EDTA. Second, for films grown in borate buffer solutions not containing EDTA, the inner barrier layer formed in the lower potential region of passivation is much thicker than the barrier layer formed in solutions containing EDTA. Not only is the barrier layer thicker in solutions not containing EDTA, but it forms in the lower passivation region and its thickness is nearly constant over the entire passive region. However, the outer precipitation layer continues to thicken with increases in formation potential. 


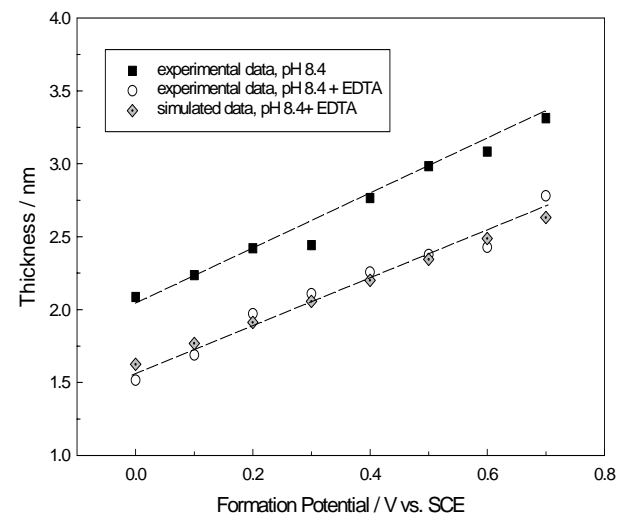

(a)

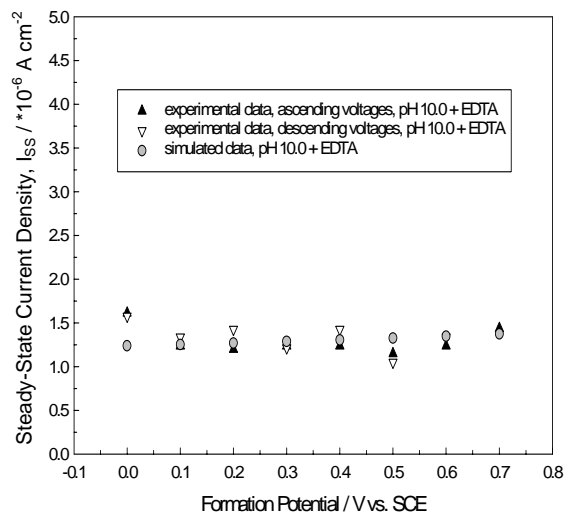

(b)

Figure III-1.4. The steady-state film thickness (a) and current density (b) for the passive film on iron. Simulated data were calculated using fundamental parameters in the Point Defect Model.

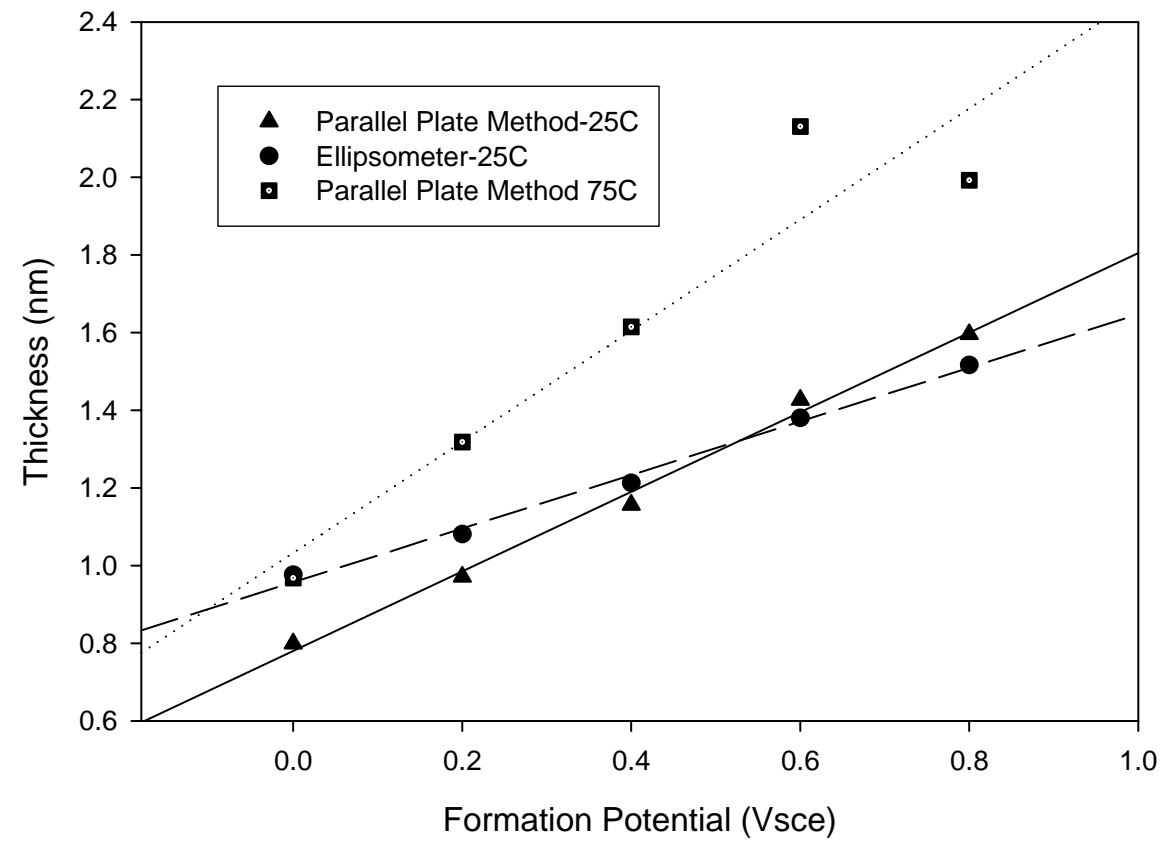

Figure III-1.5. The film thickness (as a function of formation potential) at room temperature as measured by both the parallel plate capacitance method and by the ellipsometer. The film thickness at $75^{\circ} \mathrm{C}$ is also shown. 
Table III-1.4 Individual layer and total thickness values for the oxide(s) formed on iron in borate buffer solution with and without EDTA. Values were obtained by performing a regression analysis on the $\tan (\Psi)$ and $\operatorname{Cos}(\Delta)$ to obtain a thickness or thicknesses for the oxide film(s) formed on iron. A single layer model was used to fit the data for oxides grown in the EDTA containing solution, while a double layer model was used for the fit for oxides formed in solutions not containing EDTA.

\begin{tabular}{|c|c|c|c|c|}
\hline \multirow{3}{*}{$\begin{array}{l}\text { Potential for Film Growth } \\
\text { (V vs SCE) }\end{array}$} & \multicolumn{4}{|c|}{ Oxide Thickness (nm) } \\
\hline & \multicolumn{2}{|c|}{ Without EDTA } & \multicolumn{2}{|c|}{ With EDTA } \\
\hline & $\begin{array}{c}\text { Individual Layer } \\
\text { (Outer/Inner) }\end{array}$ & Total & $\begin{array}{c}\text { Individual Layer } \\
\text { (Outer/Inner) }\end{array}$ & Total \\
\hline $0.2 \mathrm{~V}$ & $\begin{array}{l}2.99 \\
147\end{array}$ & 4.46 & $\begin{array}{l}\text { N/A } \\
0.98\end{array}$ & 0.98 \\
\hline \multirow{2}{*}{$0.4 \mathrm{~V}$} & 3.00 & \multirow{2}{*}{5.01} & N/A & \multirow{2}{*}{1.08} \\
\hline & 2.01 & & 1.08 & \\
\hline $0.6 \mathrm{~V}$ & $\frac{3.04}{2.31}$ & 5.35 & $\begin{array}{ll}\text { N/A } \\
121\end{array}$ & 1.21 \\
\hline $0.8 \mathrm{~V}$ & $\begin{array}{l}3.03 \\
3.10\end{array}$ & 6.13 & $\begin{array}{l}\text { N/A } \\
1.52\end{array}$ & 1.52 \\
\hline
\end{tabular}

\section{III-1.1c. XPS Work on Iron Oxide}

To begin, a spectrum analysis of the native oxide on iron was made using the XPS at the Penn State MRI laboratory. The sample appeared to contain the C 1s peak. To account for any shifts in the binding energy, the spectrums were calibrated using the $\mathrm{C} 1 \mathrm{~s}$ peak. Since the thickness of the iron oxide layer was desired for this sample, the peaks corresponding to iron were identified. As can be seen in Figure III-1.6 (blown up region of $\mathrm{Fe} 2 \mathrm{p}$ region), there was a splitting in the $\mathrm{Fe} 2 \mathrm{p}_{3 / 2}$ and it was shifted slightly from the typical binding energy (BE) value of $707 \mathrm{eV}$ for iron. This shift (and splitting) can be attributed to the presence of both elemental and ionized iron. By using curve fitting software, the $\mathrm{Fe} 2 \mathrm{p}_{3 / 2}$ peak was convoluted into three curves representing the $\mathrm{Fe}^{\mathrm{o}}, \mathrm{Fe}^{2+}$, and $\mathrm{Fe}^{3+}$. The combination of the three curves yield the $\mathrm{Fe} 2 \mathrm{p}_{3 / 2}$ peak shown in figure III1.6. Next, by normalizing the integrated area below the peak, the relative amounts of the different Fe species were calculated. Table III-1.3 lists the values for the two runs made on the sample. The values were then substituted into equation 30 , where $\lambda_{\text {Fe2p }}$ (maximum escape depth) was assumed to be approximately 15 angstroms and $\mathrm{K}=2$ (sensitivity factor for iron). The "I" in equation 30 is the intensity of the recorded signal (subscript denotes the species) and the fraction represents the fractional amount of iron present, either in the 
ionic state in the oxide $(\mathrm{FeO})$ or as pure iron in bulk. From this equation, the thickness was calculated to be somewhere between 19-27 angstroms, which agrees with values obtained in the literature.

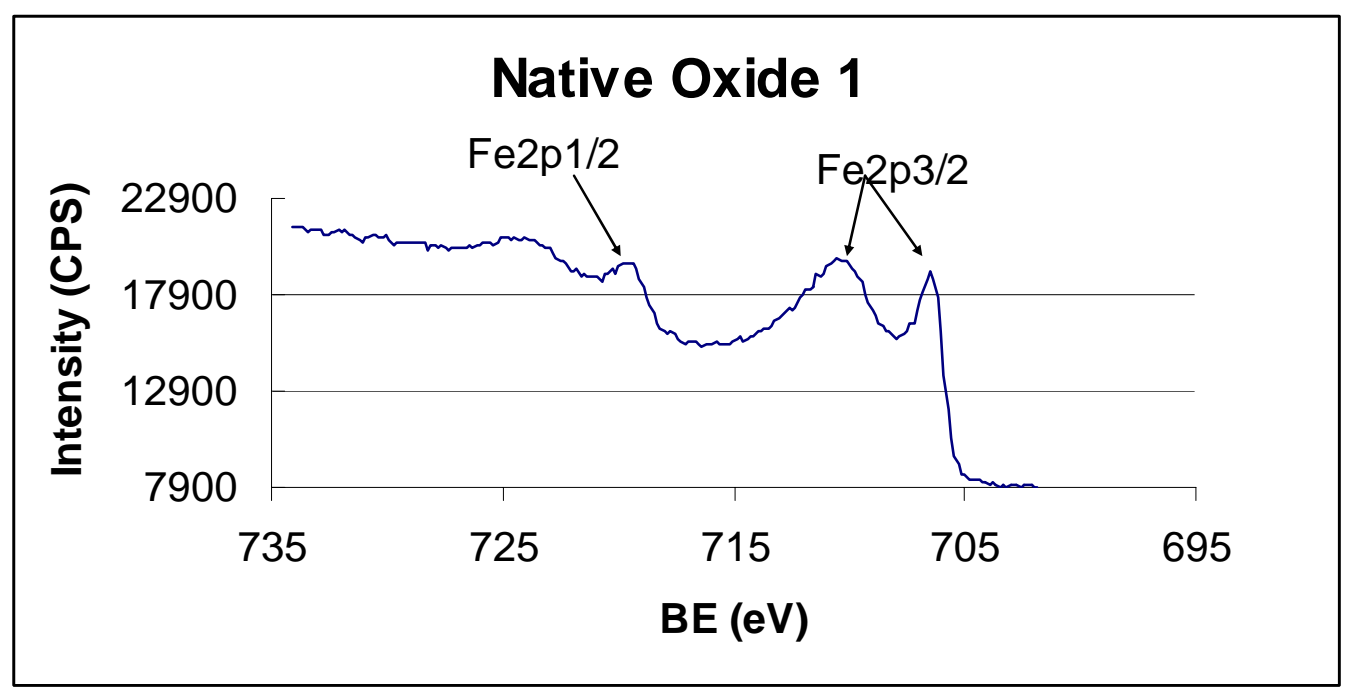

Figure III-1.6. XPS spectrum in the Fe2p region. Notice the splitting of the Fe2p3/2 peak.

Table III-1.3. Table displaying relative amounts of iron species present in Fe $2 \mathrm{p} 3 / 2$ peak. The total amount of ionized iron present (used in equation 1) is found by adding the relative amounts of $\mathrm{Fe}^{2+}+\mathrm{Fe}^{3+}$.

\begin{tabular}{|l|l|l|l|}
\hline Species Present & Integrated Area for Run 1 & \multicolumn{2}{|l|}{ Relative Amounts Present } \\
\cline { 3 - 4 } & & Run 1 & Run 2 \\
\hline $\mathrm{Fe}^{\mathrm{O}}$ & 1413.7 & $28 \%$ & $43.3 \%$ \\
\hline $\mathrm{Fe}^{2+}$ & 710.0 & $49 \%$ & $41.7 \%$ \\
\hline $\mathrm{Fe}^{3+}$ & 712.0 & $23 \%$ & $15 \%$ \\
\hline
\end{tabular}

thickness $=t=\lambda_{F e 2 p} * \ln \left[K \frac{I_{F e O}}{I_{F e}}+1\right]$

So far, measurements have been performed on samples with oxide layers grown in borate buffer solutions (containing EDTA) at three potentials in the passive range. Analysis has only been performed on the native oxide layer to verify if this method will be useful. The anaylsis shows the thickness to be in good agreement with other types of measurements. If this method provides the relative amounts of iron cations $(2+$ or $3+)$, 
the information can be incorporated into the Curve Fitting Algorithm to better account for the charged species present in the oxide layer.

\section{Issues and Concerns:}

The data quite obviously shows the presence of both atomic iron and iron cations existing near the surface. Because the ionization levels of $\mathrm{Fe}^{2+}$ and $\mathrm{Fe}^{3+}$ are very close, it is somewhat difficult to differentiate between the two ionized species. However, the shapes of the peaks strongly indicate the presence of more than one oxidation state. Therefore, a thorough analysis of the data should allow us to distinguish between the two species to a certain degree of accuracy. In addition, we will analyze the concentration of oxygen as a function of depth beneath the surface. This will provide further clues as to the oxidation states of iron and the oxygen vacancy concentrations as a function of distance across the film.

\section{III-1.2 Obtaining Kinetic Information for Passive Film Growth via Curve Fitting}

This section describes the algorithm used in Data Fit to fit the derived impedance equation to the data. Fitting the data to the equation yields the values for the kinetic parameters, which are variables in the algorithm. The code for the impedance algorithm is provided below. There are nine equations (conditions) with ten unknowns (variables). A list displaying what each condition and variable represents is shown below. It is through this algorithm that the kinetic parameters in table III-1.2 are extracted.

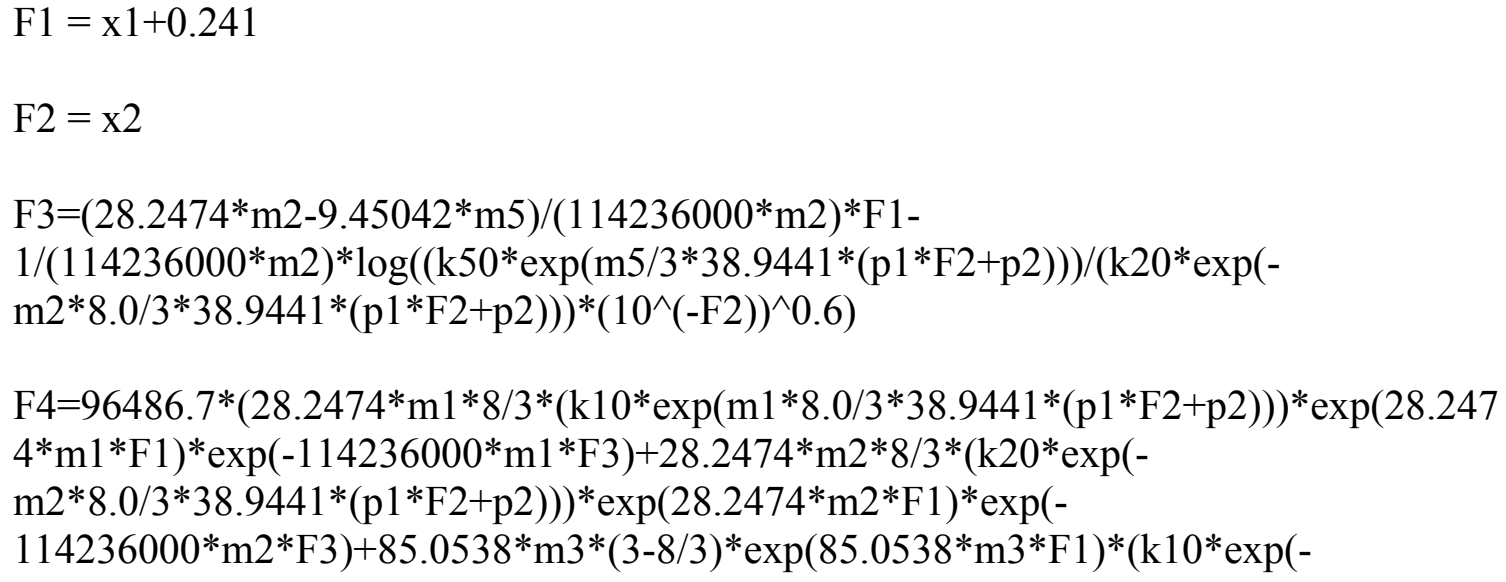




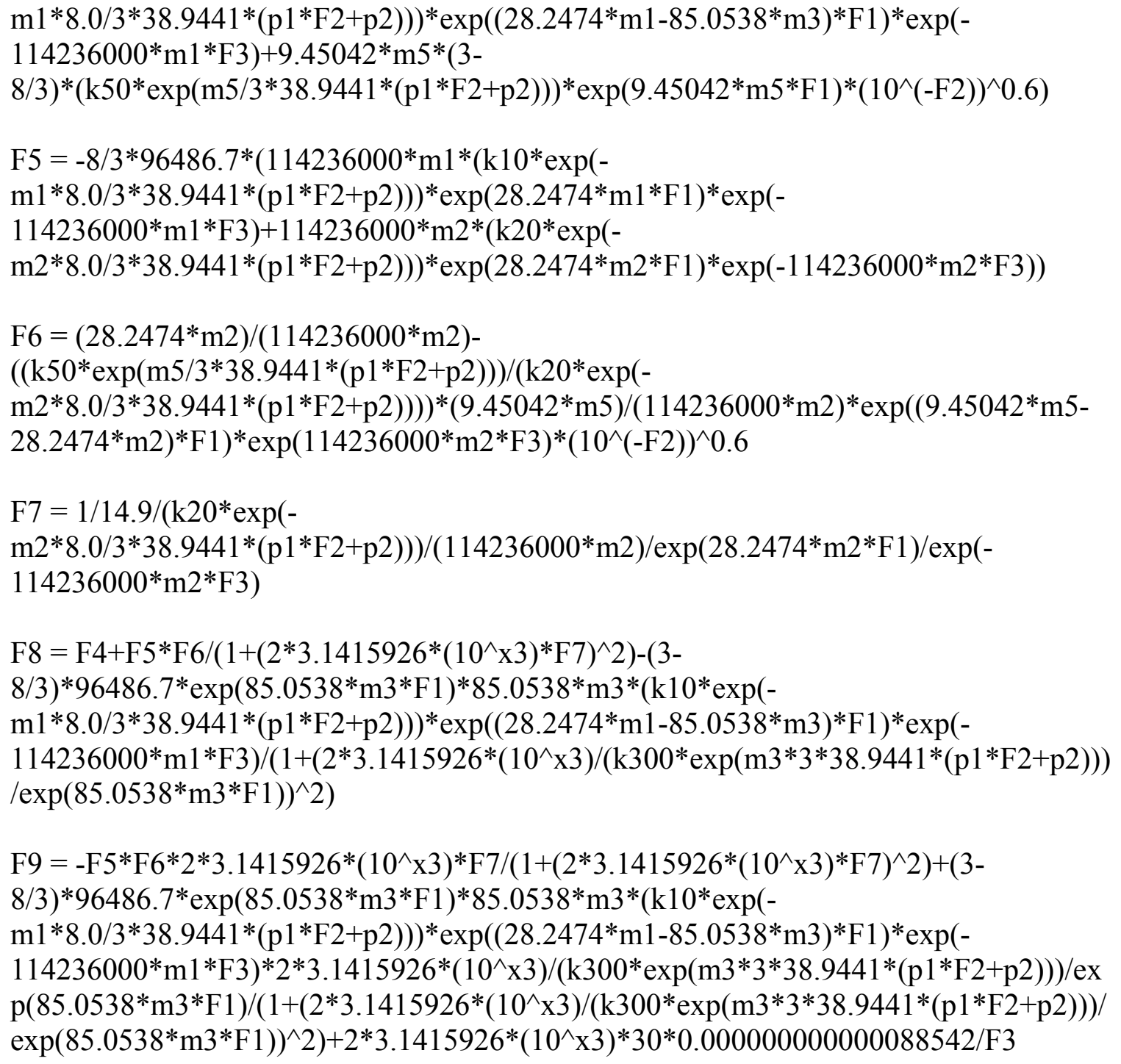

\section{Conditions:}

F1 = potential (SHE scale)

$\mathrm{F} 2=\mathrm{pH}$

$\mathrm{F} 3=$ film thickness equation

$\mathrm{F} 4=\partial \mathrm{I} / \partial \mathrm{V}$

$\mathrm{F} 5=\partial \mathrm{I} / \partial \mathrm{L}$

$\mathrm{F} 6=\phi_{2}$ (equation 10 above) 
$\mathrm{F} 7=\tau_{2}$ (equation 11 above)

$\mathrm{F} 8$ = real component of the admittance

F9 = imaginary component of the admittance

$\mathrm{Y}=\mathrm{y}($ object function) $=$ real/imaginary component of the impedance (the equation for the real component is shown above, simply replace (F8) by (-F9) in the numerator for the imaginary component)

Variables:

$\mathrm{m} 1=\alpha_{1}$

$\mathrm{m} 2=\alpha_{2}$

$\mathrm{m} 3=\alpha_{3}$

$\mathrm{m} 5=\alpha_{5}$

$\mathrm{k} 100=$ standard rate constant for reaction 1

$\mathrm{k} 200=$ standard rate constant for reaction 2 $\mathrm{k} 300$ = standard rate constant for reaction 3 $\mathrm{k} 500=$ standard rate constant for reaction 5

$\mathrm{p} 1=\beta$

$\mathrm{p} 2=\phi^{\circ}$

$\mathrm{m}=\alpha$

$\mathrm{e}=\varepsilon=$ electric field strength in the film

e1 $=$ dielectric constant for iron oxide.

\section{Issues and Concerns:}

The magnitude of the relative error in some of the fits is quite large at high frequencies. We found that this is most likely due to the high number of parameters being used to fit the equation ( 9 parameters). The high number of parameters can give rise to a very large number of "solutions" or "fits". For this reason, we are going to use experimental techniques to measure the film dissolution rate. From this rate, we can calculate two of the rate constants used to fit the equation to the data. We are researching methods to measure some of the other parameters in hopes of decreasing the number of "solutions" to the problem. This should provide us with information to allow for much more accurate fits in the high frequency regions. We are also looking into another data fitting software package that uses different fitting algorithms than the more popular commercially available ones. We hope to find that these different algorithms will allow better fitting. 


\section{III-1.3 Experimental Setup to Obtain Data for DFA}

The development of the Damage Function Analysis (DFA) is described in detail in another section of this report. Only the experimental set-up for obtaining data to be used in the development of DFA is being described here. However, it should be mentioned that the original DFA requires data for the number of pits/area as a function of the depth below the surface of the sample [2]. This view comes from the idea that pits nucleate and grow at different times and rates and the pits can either be active (growing) or dead (repassivated). Because pits nucleate and grow at different times and rates, there should be a trend showing that the number of pits/area decreases with depth. If the number of pits/area surpasses a critical value at a particular depth, failure can occur. By using data on the number of pits/area as a function of depth and time, DFA can predict failure due to pitting corrosion.

Taking this perspective can lead to some problems as depicted in figure III-1.7. It can easily be seen that the number of pits/area at the various depths does not definitively follow a trend where there is a higher density of pits at the surface which decreases with depth below the surface. This apparent inconsistency arises due to the fact that pits grow in complex shapes as the pit front proceeds deeper beneath the surface. To rectify this problem, it has been suggested that the DFA account for the depth of the deepest pit as function of time, temperature, and aggressive ion concentration.

To obtain this data, ASTM A516 steel will be placed in solutions for various times-1 week, 2 week, 1 month, and 4 month times have been suggested. Three temperatures $\left(25^{\circ} \mathrm{C}\right.$ to $90^{\circ} \mathrm{C}$ ), potentials (from the corrosion potential to the upper end of the passive region), $\mathrm{pH}$ 's (from 7 to 14), and aggressive ion concentrations will be analyzed. For each condition, nine samples will be used to obtain the data. These samples can be measured simultaneously. After the set time period, the samples will be removed from the solution and the maximum pit depth for each of the nine samples will be measured. This information will then be used as the input data for the DFA as discussed in the DFA section of this report. 


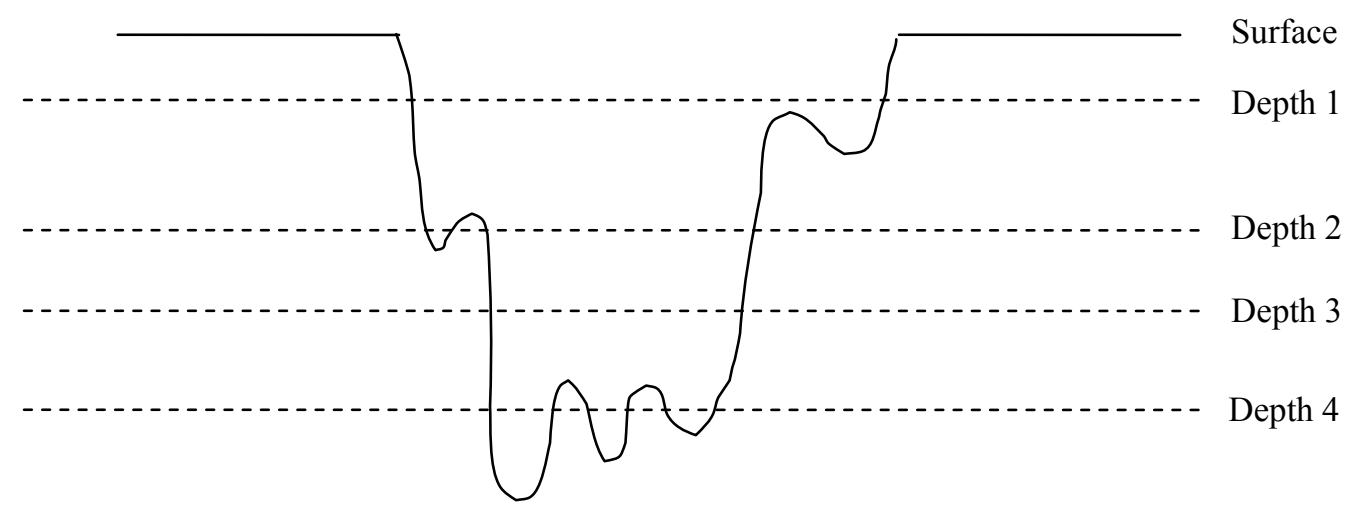

Figure III-1.7. Due to the complex shape of a pit, the number of pits/area as a function of depth does not appear to decrease as would be expected. Depth 1 shows 1 pit, depth 2 shows 2 pits, depth 3 shows 1 pit, and depth 4 shows 3 pits.

Based on Extreme Value Statistics, DFA can predict damage/failure due to pitting corrosion. This is a statistical approach based on empirical data. However, by assuming that vacancy coalescence in the film is the mechanism responsible for pit nucleation [5], a deterministic-probabilistic DFA can be developed. The terms describing the vacancy coalescence have been derived and are based on the kinetic parameters extracted from the PDM. Applying the kinetic parameters from the experiments on iron, the critical vacancy density for pit formation can be calculated. This information, if shown to be correct, can prove to be a monumental find in the understanding of pitting corrosion.

Issues and Concerns: $\quad$ None

\section{III-1 References}

1. D.D. Macdonald, M. Uriquidi-Macdonald, J. of Electrochemical Society, 137, 2395 (1990).

2. G. Engalhardt, D.D. Macdonald, Corrosion, 54, 469 (1998).

3. J. Liu, D.D. Macdonald, J. of Electrochemical Society, 148, B425 (2001).

4. D. D. Macdonald, Pure Appl. Chem., 71, 951 (1999).

5. D.D. Macdonald, J. of Electrochemical Society, 139, 3434 (1992). 


\section{III-2. Task: The Role of Chloride Ion in Passivity Breakdown on Nickel}

\section{Task Status}

\section{III-2.1 Experimental set up}

All electrochemical studies were carried out in a three-electrode, PTFE electrochemical cell. A platinum counter electrode and a saturated calomel reference electrode (SCE) were used to carry the cell current and to measure the potential of the working electrode, respectively. All electrode potentials are referred to the SCE scale. Pure Ni (99.99 \%, Alfa Aesar) was used as the working electrode. The nickel rod was mounted in epoxy resin with one end of $1-\mathrm{cm}^{2}$ area being exposed to the solution. Prior to performing an experiment, the exposed nickel was polished with successively finer SiC papers ranging from 600 to 2000 grit. The solution used in this study was the deaerated, $\mathrm{pH}=8.5$ borate buffer solution prepared by mixing $0.2 \mathrm{M}$ boric acid and 0.05 $\mathrm{M}$ citric acid with $0.1 \mathrm{M}$ tertiary sodium phosphate solution [1]. The concentration of $\mathrm{Cl}^{-}$ ion was established by adding the requisite amount of $\mathrm{NaCl}$ to the solution. All experiments were performed at ambient temperature $\left(22 \pm 2{ }^{\circ} \mathrm{C}\right)$. The working electrode was initially reduced potentiostatically at $-1.0 \mathrm{~V}_{\mathrm{SCE}}$ for $30 \mathrm{~min}$. to remove any air-formed oxide on the surface, and then a passive film was grown potentiodynamically by ramping the applied potential from $E_{\text {corr }}$ to $400 \mathrm{mV}_{\mathrm{SCE}}$ at a sweep rate of $0.5 \mathrm{mV} / \mathrm{sec}$. After reaching $400 \mathrm{mV}_{\mathrm{SCE}}$, that potential was maintained for 2 hours before initiating the measurements of interest.

Electrochemical experiments were performed using a Solartron 1287 Electrochemical Interface. Electrochemical impedance data were recorded with a Solartron 1255B Frequency Response Analyzer (FRA), using an excitation voltage of 10 $\mathrm{mV}$ (peak-to-peak). Capacitance data for Mott-Schottky analysis were obtained at a constant frequency of $1 \mathrm{kHz}$.

\section{Issues and Concerns: None}




\section{III-2.2 Applying Mott-Schottky Analysis and EIS to Study Passivity Breakdown}

The polarization behavior of nickel in the borate buffer solution with and without chloride present is shown in Figure III-2.1. Chloride is seen to induce passivity breakdown at progressively lower potentials as the chloride concentration increases. The voltage at which the measurements were made in this study $\left(400 \mathrm{mV}_{\mathrm{SCE}}\right)$ is about $50 \mathrm{mV}$ more negative than the apparent breakdown voltage for the most concentrated chloride solution.

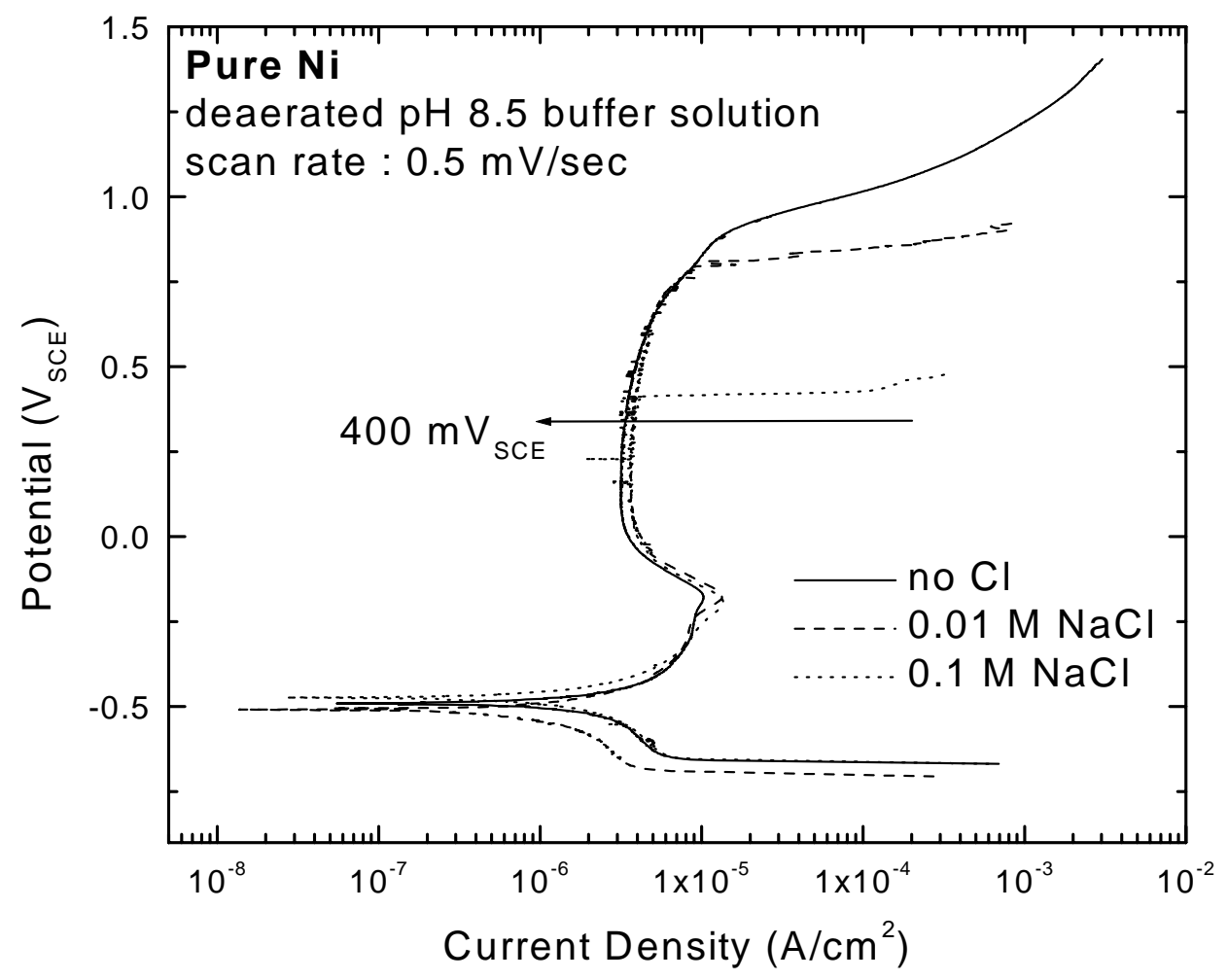

Figure III-2.1 Polarization curves for nickel in borate buffer solution with or without chloride ion.

Figure III-2.2 shows the effects of $\left[\mathrm{Cl}^{-}\right]$on potentiostatic transients (chronoamperograms) for $\mathrm{Ni}$ obtained by stepping the potential from the open circuit value to $400 \mathrm{mV}_{\mathrm{SCE}}$. The transients are typical in form, in that the current decreases with 
time. At any given time after imposition of the potential step, the passive current density was found to be slightly greater with increasing chloride concentration. Thus, the chloride ion catalyzes the transfer of charge across the passive film slightly. It is notable that meta-stable pitting events are observed for the specimen immersed in the solution containing $0.1 \mathrm{M} \mathrm{NaCl}$, indicating that passivity breakdown occurs under the prevailing conditions.

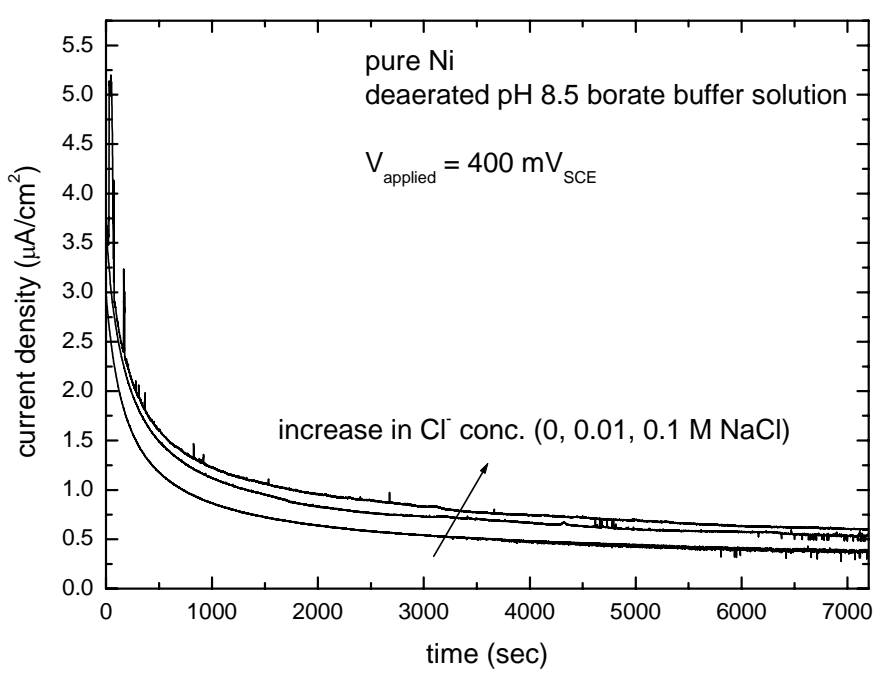

Figure III-2.2 Current transient curves of $\mathrm{Ni}$ in chloride-containing, deaerated, $\mathrm{pH} 8.5$ borate buffer solutions measured at $400 \mathrm{mV}_{\mathrm{SCE}}$. The concentration of $\mathrm{Cl}^{-}$was controlled from 0 to $0.1 \mathrm{M}$ by adding $\mathrm{NaCl}$ to the solution.

From previous studies on the electronic properties of the passive film on $\mathrm{Ni}$ [2], the barrier layer was found to be p-type semiconductor within the passive range, due to a preponderance of cation vacancies $\left(V_{N i}^{\chi^{\prime}}\right)$ in the lattice. The abundance of cation vacancies over oxygen vacancies $\left(V_{o}^{*}\right)$ is rationalized by the fact that the energy of

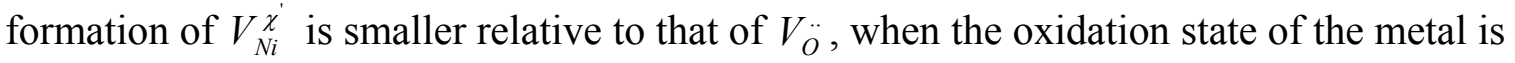
small ( $\chi=2$ for $\mathrm{Ni}$ in the passive state) [3].

To investigate the effects of $\mathrm{Cl}^{-}$on the electronic properties of passive film, Mott-Schottky analyses were performed at a fixed frequency of $1 \mathrm{kHz}$. The interfacial 
capacitance, $C$, is obtained from $C=1 / \omega Z$ ", where $\omega$ is the angular frequency and $Z$ " is the imaginary part of impedance. Assuming that the capacitance of the Helmhotlz layer can be neglected (because it is much larger than the space charge capacitance of the passive film and the capacitances are in series), the measured capacitance $C$ is equal to the space charge capacitance, $C_{s c}$. According to Mott-Schottky theory [4] the space charge capacitance of a p-type semiconductor is given by Equation (1),

$$
\frac{1}{C^{2}}=\frac{-2}{\varepsilon \varepsilon_{0} e N_{A}}\left(V-V_{f b}-\frac{k T}{e}\right)
$$

where $\varepsilon$ is the dielectric constant of the oxide, $\varepsilon_{0}$ is the vacuum permittivity, $e$ is the charge of an electron, $N_{A}$ is the acceptor concentration in the film, $V$ is the applied potential, $V_{f b}$ is the flat band potential, and $k$ is the Boltzmann constant. Thus, for a ptype semiconductor, $C^{-2}$ versus $V$ should be linear with a negative slope that is inversely proportional to the acceptor concentration.

Figure III-2.3 shows the effects of $\mathrm{Cl}^{-}$on Mott-Schottky plots for passive nickel. The Mott-Schottky plots are characteristic of p-type semi-conducting passive film and the acceptor levels in the film, calculated from the slopes and using $\varepsilon=12[5,6]$, are presented in Figure III-2.4. It is clear that the acceptor level within the passive film increases with increasing $\mathrm{Cl}^{-}$concentration. Previously, Macdonald et al. [7] and Liu et al. [8] reported that there exists a correlation of high fidelity between the crystallographic defect structure and the electronic structure for passive films on pure metals, which shows that the defects (donors for n-type semiconductors and acceptors for p-type semiconductors) are themselves the dopants, including cation vacancies (p-type), anion vacancies (n-type), and cation interstitials (n-type). Accordingly, the acceptor species in Figure III-2.4 are considered to be cation vacancies $\left(V_{N i}^{\chi^{\prime}}\right)$ in the passive film. These results demonstrate that $\mathrm{Cl}^{-}$in $\mathrm{pH} 8.5$ borate buffer solution increases the concentration of the metal vacancy in the passive film on $\mathrm{Ni}$. However, it should be noted that chloride was present in the solution during the film formation period, and hence the defect 
structure may have been "built-in" during the growth process, rather than being induced by the interaction of chloride ion with the outer surface of the film.

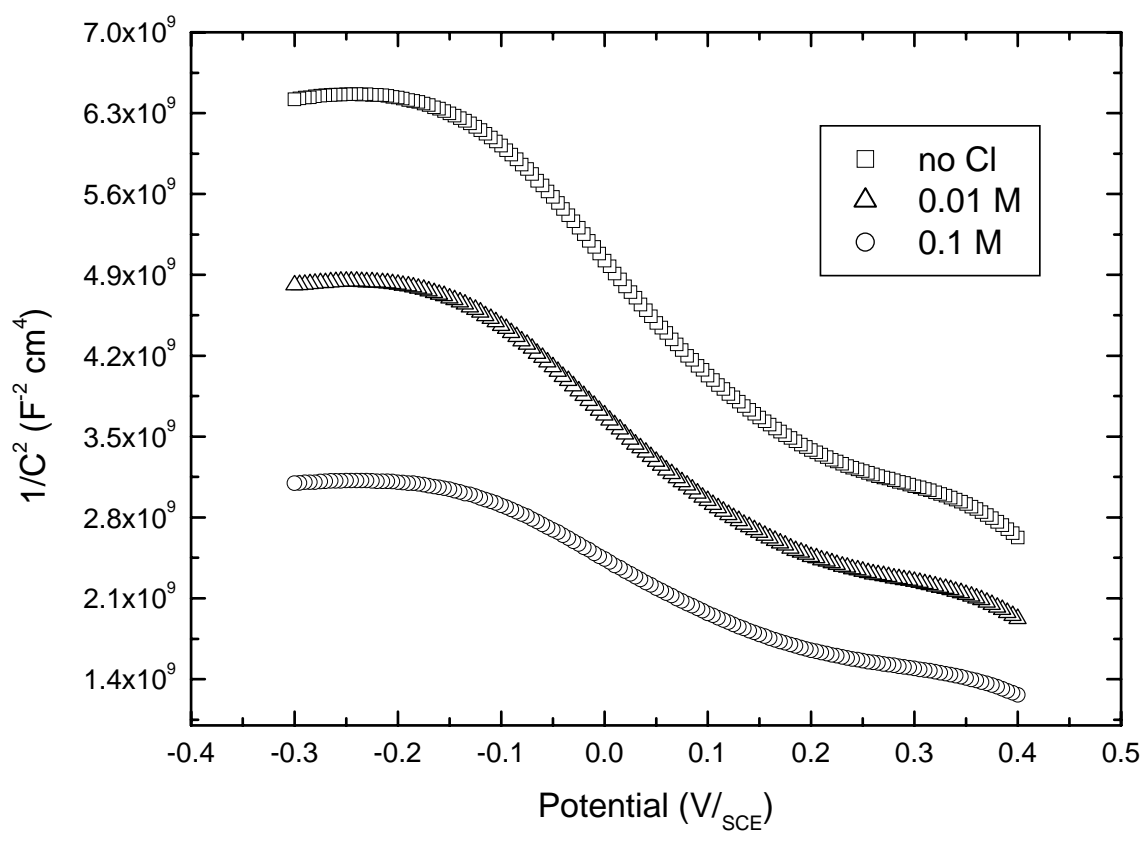

Figure III-2.3 Mott-Schottky plots for the passive film formed on $\mathrm{Ni}$ in deaerated, $\mathrm{pH} 8.5$ borate buffer solution at $400 \mathrm{mV}_{\mathrm{SCE}}$ for 2 hours with different concentration of $\mathrm{Cl}^{-}$ions measured at a frequency of 1 $\mathrm{kHz}$. 


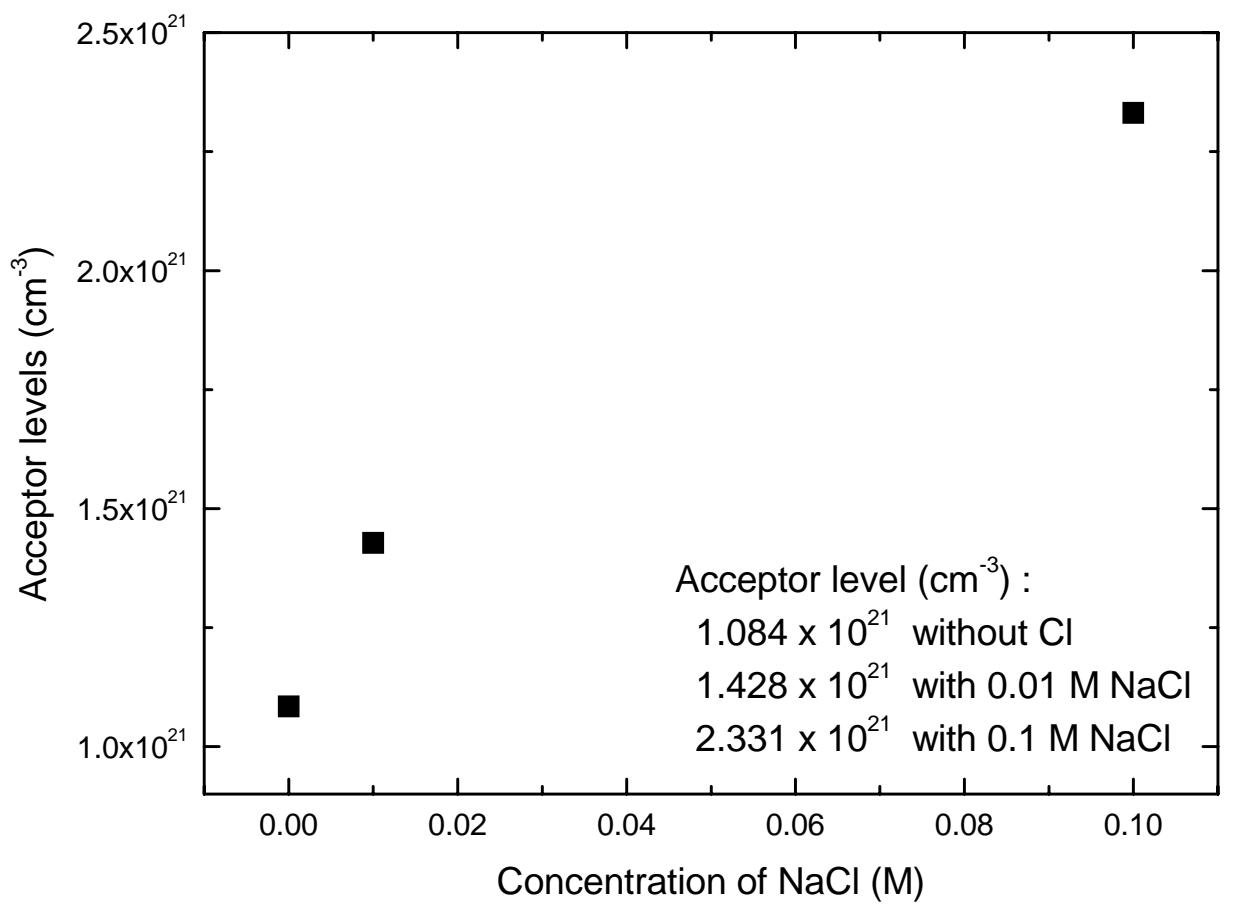

Figure III-2.4 Acceptor levels calculated from the slopes of the Mott-Schottky plots in Fig. 6.

To examine the effect of adding $\mathrm{Cl}^{-}$to the solution after passivity had been established in the absence of this species, another set of Mott-Schottky analyses were performed. Thus, the passive film was initially grown in $\mathrm{Cl}^{-}$-free, $\mathrm{pH} 8.5$ borate buffer solution at $400 \mathrm{mV}_{\text {SCE }}$ for 2 hours. Then, $\mathrm{NaCl}$ was added to the solution to yield a chloride concentration of $0.1 \mathrm{M}$. Mott-Schottky plots were then obtained as a function of time (Figure III-2.5), and the calculated acceptor levels, shown in Figure III-2.6, were calculated. These data show that chloride induces an increase in the concentration of cation vacancies in the barrier layer after the film is formed. This finding is inconsistent with chloride modifying the film growth process, but it is consistent with the presence of a chloride-catalyzed cation vacancy generation process at the barrier layer/solution interface. The nature of the chloride-catalyzed cation vacancy generation process is such that it results in a linear increase in the cation vacancy concentration with time, at least 
over the first four hours of exposure to chloride. We should note, that Mott-Schottky analysis presumably yields the acceptor concentration averaged over all sites on the surface. However, localized corrosion occurs at sites that occupy only a very small fraction of the total area, so that the level of enhancement of the cation vacancy concentration at the breakdown sites is presumably much greater than indicated in Figures III-2.4 and III-2.6.

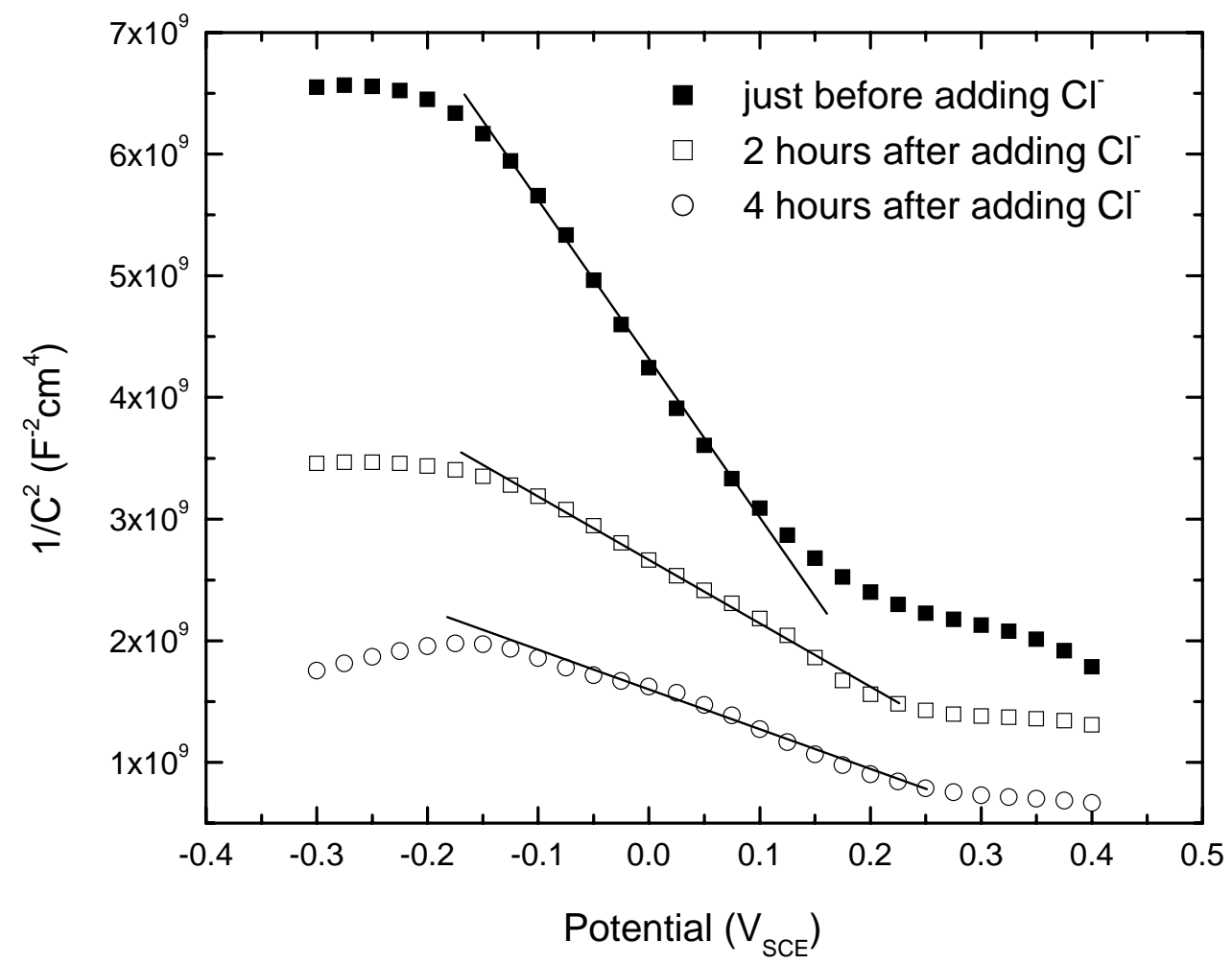

Figure III-2.5 Mott-Schottky plots for the passive film formed on $\mathrm{Ni}$ in deaerated, $\mathrm{pH} 8.5$ borate buffer solution at $400 \mathrm{mV}_{\mathrm{SCE}}$, as calculated from capacitance data measured at a frequency of $1 \mathrm{kHz}$. The passive film was initially grown in chloride-free solution for 2 hours and then $\mathrm{NaCl}$ was added to the solution to adjust the total concentration of $\mathrm{Cl}^{-}$to $0.1 \mathrm{M}$. 


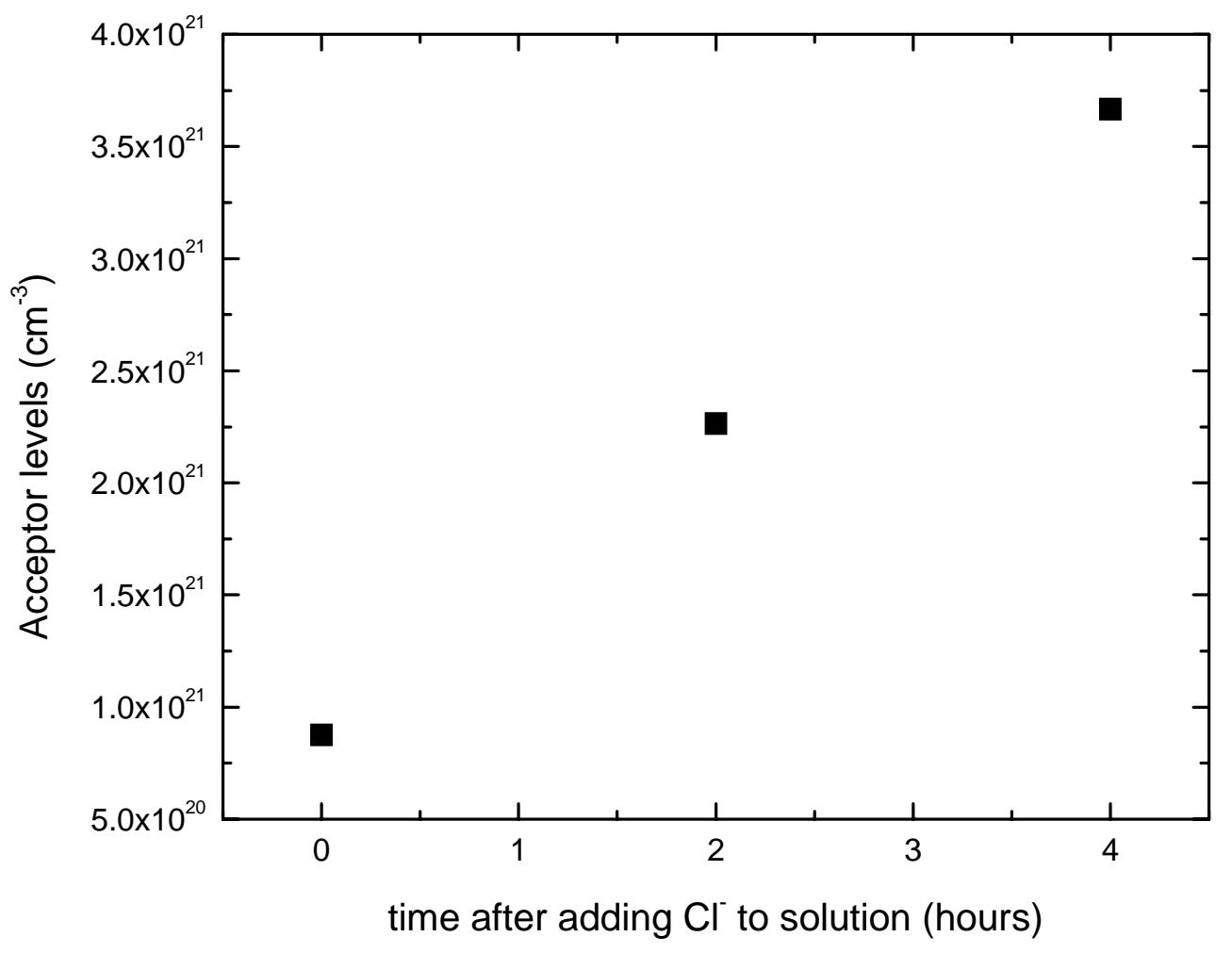

Figure III-2.6 Acceptor levels calculated from the slopes of the Mott-Schottky plots in Fig. 8.

The final issue that we wish to explore is the fundamental nature of the processes that occur at the barrier layer/solution interface that result in an increase in the cation vacancy concentration within the barrier layer, when the system is exposed to chloride ion. This issue was explored using electrochemical impedance spectroscopy (EIS) to measure the impedance of the $\mathrm{Ni} / \mathrm{NiO} /$ solution interphase over a wide frequency range and by then fitting a reduced PDM to the data, in order to extract values for important parameters in the model. The fitting was done using an optimization program from Oakdale Engineering, www.curvefitting.com. Therefore, the reduced PDM was generated by ignoring reactions involving metal interstitials [Reactions 2 and 5, Figure II2.1]. Metal interstitials would dope the film n-type in electronic character and no hint of 
n-type behavior is observed at $400 \mathrm{mV}_{\mathrm{SCE}}$. A full account of the fitting procedure will be published at a later date. The impedance data were found to correctly transform according to the Kramers-Kronig integral transforms, thereby demonstrating that the system conforms to the constraints of linear systems theory [9].

Figure III-2.7 shows experimental impedance data for passive $\mathrm{Ni}$, together with the model fit for the reduced PDM. The fit is judged to be very good for the three systems explored $(0,0.01$, and $0.1 \mathrm{M} \mathrm{NaCl})$. Values for selected parameters determined from the optimization are summarized in Table III-2.1. Of the parameters listed, only the electric field strength was assumed on the basis of previous work [10]; no credible reason exists within the PDM for believing the electric field strength across the barrier layer should depend upon the chloride concentration. Of the parameters listed, $k_{i}^{00}$ represents the standard rate constant and $\alpha_{i}$ is the transfer coefficient of $i$-th reaction in Figure II-2.1. Examination of the data in Table III-2.1 shows that there is no systematic dependence of any of the kinetic parameters listed in Table III-2.1 on chloride concentration, except for the rate constant for the cation ejection Reaction 4, Figure II-2.1. In this case, even though the experimental data are not extensive and assuming that the kinetic order must be an integer, the rate constant evidently increases in a first order fashion with chloride concentration (Table III-2.1). Because this reaction is envisioned to be elementary in nature, the kinetic order is equivalent to the molecularity and hence we conclude that the reaction involves a single chloride species. This finding demonstrates unequivocally that the role of chloride in inducing passivity breakdown on nickel is catalysis of the cation vacancy generation reaction at the barrier layer/solution interface, as envisioned in the Point Defect Model (Figure II-2.3). 


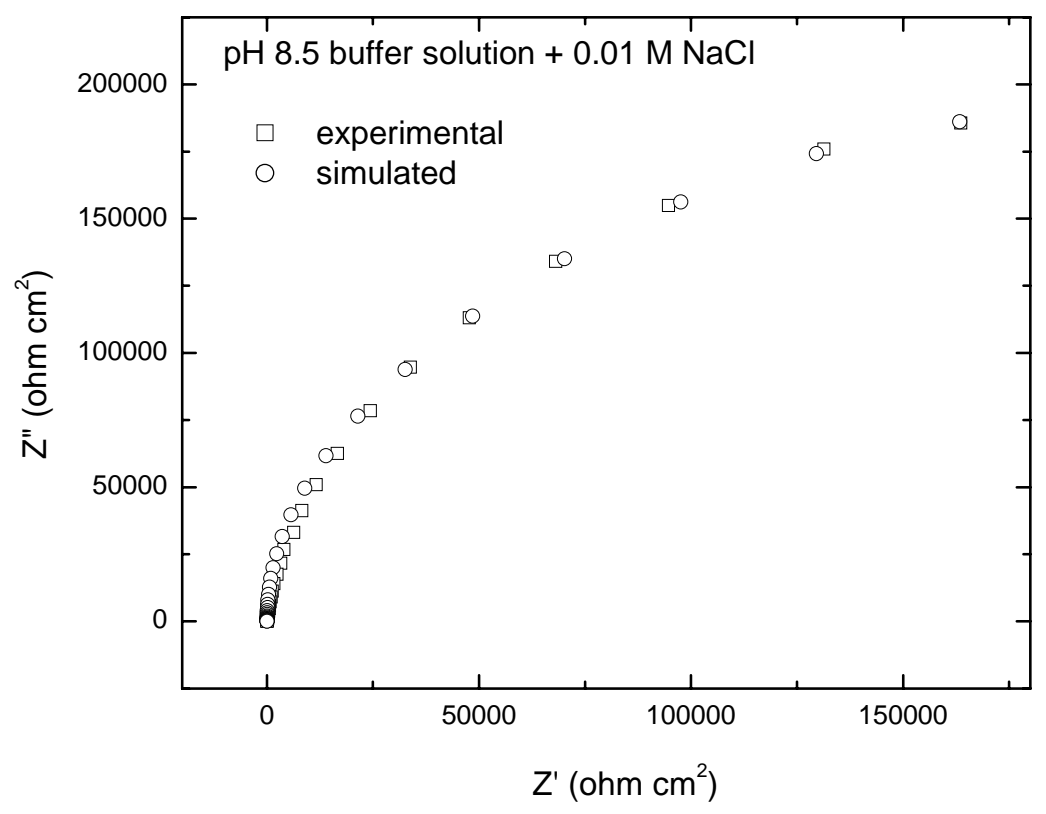

Figure III-2.7 Nyquist plot for the passive film formed on Ni in chloride-containing, pH 8.5 borate buffer solutions at $400 \mathrm{mV}_{\mathrm{SCE}}$ for 2 hours, together with the model fit by the PDM. The concentration of $\mathrm{Cl}^{-}$in the solution was (a) $0 \mathrm{M}$, (b) $0.01 \mathrm{M}$ and (c) $0.1 \mathrm{M}$. 


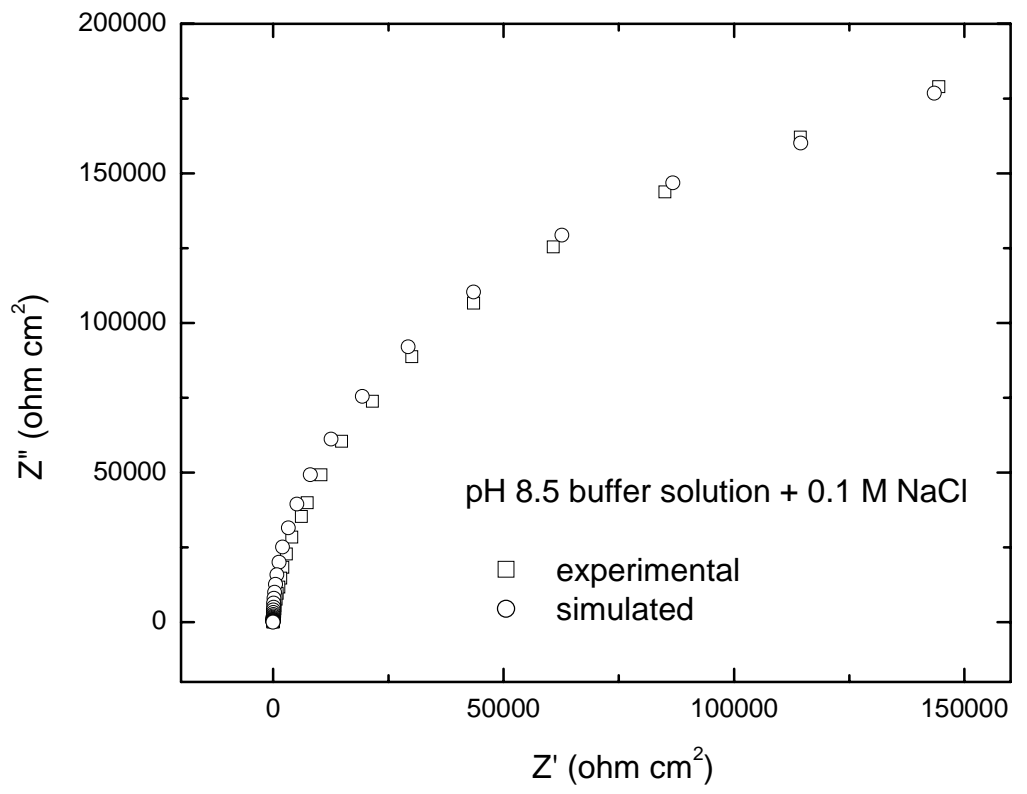

Figure III-2.7 Kinetic plot for cation vacancy generation at the barrier layer/solution interface (Reaction 4, Figure II-2.1). The line is drawn for a first order dependence of the rate of the reaction on chloride concentration while the points with error bars are experimental data.

Table III-2.1 Values for various parameters in the Point Defect Model, as determined by fitting the PDM to the experimental electrochemical impedance data.

\begin{tabular}{|c|c|c|c|}
\hline Parameter & $0 \mathrm{M} \mathrm{NaCl}$ & $0.01 \mathrm{M} \mathrm{NaCl}$ & $0.1 \mathrm{M} \mathrm{NaCl}$ \\
\hline Electric Field Strength $\hat{\varepsilon}(\mathrm{V} / \mathrm{cm})$ & $1.00 \times 10^{6}$ & $1.00 \times 10^{6}$ & $1.00 \times 10^{6}$ \\
\hline Rate Constant, $k_{1}^{00}(\mathrm{~cm} / \mathrm{s})$ & $2.30 \times 10^{-12}$ & $3.19 \times 10^{-12}$ & $3.42 \times 10^{-12}$ \\
\hline Rate Constant, $k_{3}^{00}\left(\mathrm{~mol} / \mathrm{cm}^{2} \mathrm{~s}\right)$ & $2.89 \times 10^{-18}$ & $3.11 \times 10^{-18}$ & $3.03 \times 10^{-18}$ \\
\hline Rate Constant, $k_{4}^{00}\left(\mathrm{~mol} / \mathrm{cm}^{2} \mathrm{~s}\right)$ & $4.92 \times 10^{-14}$ & $3.90 \times 10^{-13}$ & $6.39 \times 10^{-12}$ \\
\hline Rate Constant, $k_{7}^{00}\left(\mathrm{~mol}^{0.4} / \mathrm{cm}^{0.2} \mathrm{~s}\right)$ & $3.00 \times 10^{-12}$ & $3.63 \times 10^{-12}$ & $3.60 \times 10^{-12}$ \\
\hline Transfer Coeff., & 0.40 & 0.39 & 0.38 \\
\hline Transfer Coeff., & 0.07 & 0.07 & 0.07 \\
\hline Transfer Coeff., & 0.19 & 0.06 & 0.1 \\
\hline
\end{tabular}


Finally, the fact that the rate constant for the passive film dissolution reaction (Reaction 7, Figure II-2.1) and the rate constants for the reactions at the metal/film interface (Reactions 1 and 3, Figure II-2.1) are essentially unaffected by chloride is inconsistent with the chloride-catalyzed film dissolution and chloride penetration models, respectively, for passivity breakdown.

Issues and Concerns: None

\section{III-2. References}

1. G. J. Shugar, T. Ballinger, Chemical Technicians' Ready Reference Handbook, $3^{\text {rd }}$ ed., McGraw Hill, New York, NY, 1990, p. 655

2. E. Sikora and D. D. Macdonald, Electrochim. Acta., 48, 69 (2002).

3. D. D. Macdonald, Pure Appl. Chem., 71, 6, 951 (1999).

4. S. R. Morrison, Electrochemistry at Semicondutor and Oxidized Metal Electrodes, Plenum Press, NY, 1980.

5. G. Barral, F. Njanjo-Eyoki, S. Maximovitch, Electrochim. Acta, 40, 2815 (1995).

6. Y. Okazaki, T. Tateishi, and Y. Ito, Materials Transactions, JIM, 38, 78 (1997).

7. 17 D. D. Macdonald, S. R. Biaggio, and H. Song, J. Electrochem. Soc., 139, 170 (1992).

8. R. P. Frankenthal and J. Kruger (eds.), Passivity of Metals, The Electrochemical Society, Princeton, NJ (1978).

9. M. Urquidi-Macdonald, S. Real, and D. D. Macdonald, Electrochim. Acta, 35(10), 1559 (1990).

10. K-S Lei, D. D. Macdonald, B. G. Pound, and B. E. Wilde, J. Electrochem. Soc., 135, 1625 (1988).

\section{III-3. Task: Crack propagation studies}

\section{$\underline{\text { Task status }}$}

\section{III-3.1 Experimental Setup}

The material used in this investigation was a commercial heat of AISI 4340 steel, which was prepared by electric furnace melting and vacuum degassing. The chemical composition is given in Table III-3.1. The as-received steel plate was $38 \mathrm{~mm}$ (1.5 in.) thick. The plate was cut first into blanks and heat-treated according to the procedures given in Table III-3.2. The yield strength was not measured, but the value was estimated to be $\sim 1,490 \mathrm{MPa}$ according to the measured hardness $(48.5 \mathrm{Rc})$ and the typical 
properties of AISI 4340 steel of this temper. Specimens were machined in the fully heattreated condition. The configuration used for monitoring the coupling current during crack propagation in the compact tension $[\mathrm{C}(\mathrm{T})]$ specimen is shown in Figure III-3.1. The specimen geometry conforms to ASTM E399 based upon $\mathrm{W}=2.997 \mathrm{~cm}$ (1.18 in.). Some specimens were prepared in the short transverse-longitudinal (S-L) orientation and the others were prepared in the longitudinal-transverse (L-T) orientation. Unless specified the test results and the subsequent discussions correspond to the tests carried out using the S$\mathrm{L}$ specimens. A separate section is dedicated to discuss the results form the tests carried out using the L-T specimens. Shallow side grooves $(5 \%$ of the specimen thickness on both sides) were machined along the crack plane to enhance plane strain and to guide the direction of the growing crack. The effect of reduced thickness (Bn) on the stress intensity was taken into account by replacing $\mathrm{B}$ by $\left(\mathrm{BB}_{\mathrm{n}}\right)^{1 / 2}$ in the equation for $\mathrm{K}_{\mathrm{I}}$, where $\mathrm{B}$ is the specimen thickness and $\mathrm{B}_{\mathrm{n}}$ is the reduced thickness at the groove. The specimens were carefully cleaned and wires were then spot-welded onto the surface. Then, the entire surface of each specimen was coated with baked-on polytetrafluoroethylene (PTFE) for electrical insulation, so that only the crack after fatigue pre-cracking was exposed to the environment. The side cathodes were then mounted and held in place by insulated clips.

TABLE III-3.1

Chemical Composition of AISI 4340 Steel

\begin{tabular}{|c|c|c|c|c|c|c|c|c|c|c|}
\hline Element & $\mathrm{C}$ & $\mathrm{Mn}$ & $\mathrm{P}$ & $\mathrm{S}$ & $\mathrm{Si}$ & $\mathrm{Cu}$ & $\mathrm{Ni}$ & $\mathrm{Cr}$ & $\mathrm{Mo}$ & $\mathrm{V}$ \\
\hline Wt. \% & 0.43 & 0.71 & 0.013 & 0.004 & 0.26 & 0.18 & 1.72 & 0.80 & 0.24 & 0.59 \\
\hline
\end{tabular}

TABLE III-3.2

Heat Treatment and Mechanical Properties of AISI 4340 Steel

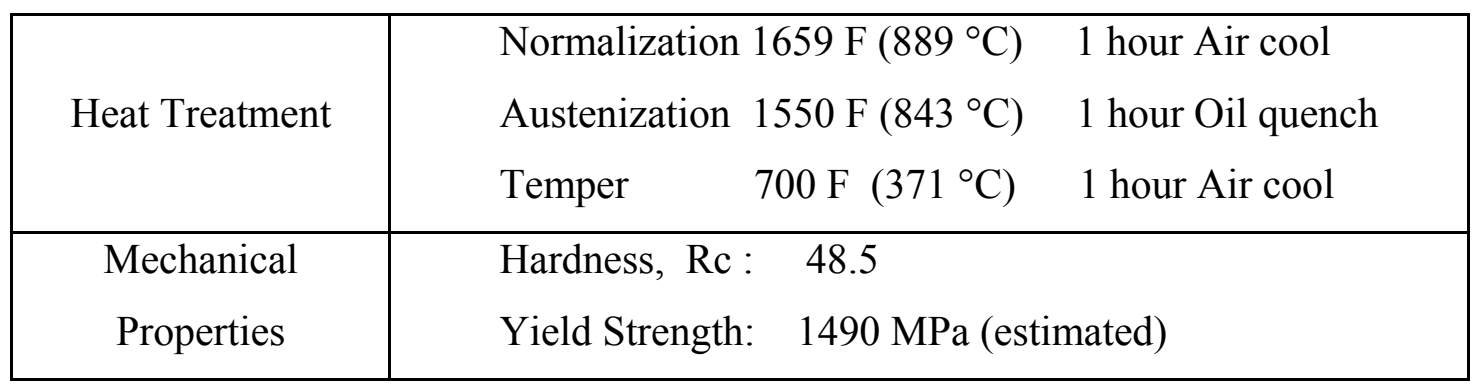




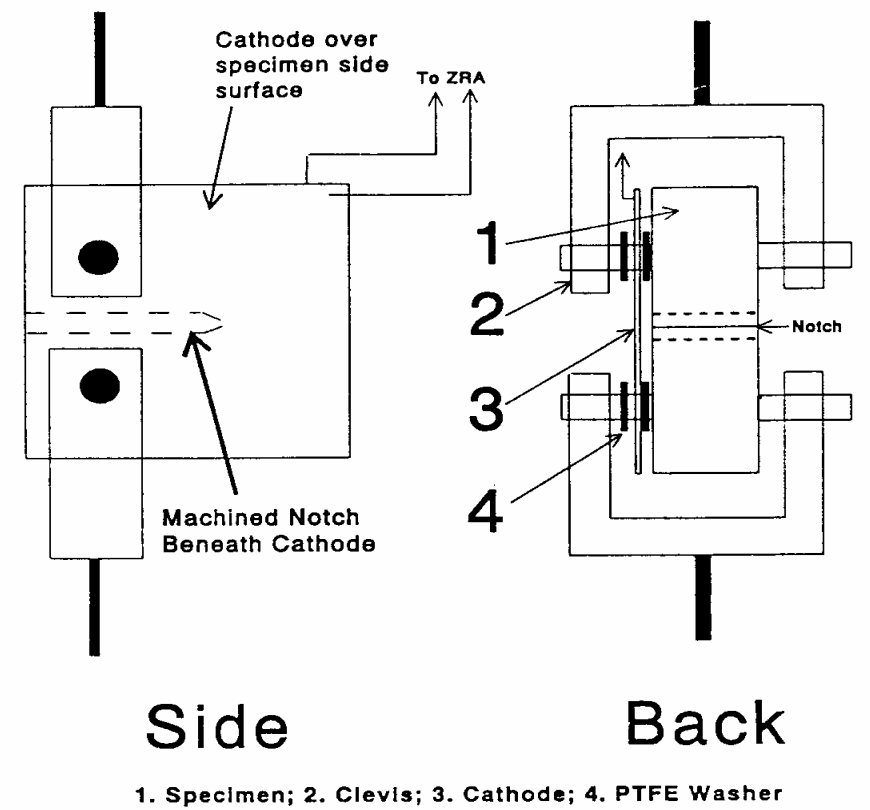

Figure III-3.1. Specimen configuration used in detecting and measuring the coupling current flowing from a crack to the external metal surface. Note that the $\mathrm{C}(\mathrm{T})$ fracture mechanics specimen is coated with PTFE to inhibit the cathodic reduction of oxygen on the specimen surface. Instead, the current flows from the crack to the side cathodes (only one shown) where it is consumed by $\mathrm{O}_{2}$ reduction. The electron current flows from the crack tip to the side cathodes via a zero resistance ammeter, which is used for its measurement.

An MTS 810 universal testing machine was used as the loading apparatus. A special tank made of a nickel-based alloy was used for conducting SCC experiments in hot, concentrated $\mathrm{NaOH}$ solution. Prior to $\mathrm{SCC}$, the $\mathrm{C}(\mathrm{T})$ specimens were pre-cracked to total crack length of $0.40 \mathrm{~W}$ to $0.42 \mathrm{~W}$ as required by ASTM E399 guidelines for load and load shedding at a frequency of $20 \mathrm{~Hz}$. After initiating and propagating the fatigue crack for $\sim 1 \mathrm{~mm}$ under $\mathrm{Kmax}=20 \mathrm{MPa} \sqrt{\mathrm{m}}$ with $\mathrm{R}=0.1$, the maximum applied stress intensity was reduced to $<10 \mathrm{ksi} \sqrt{i n}_{\text {. }}$. (11 $\left.\mathrm{MPa} \sqrt{\mathrm{m}}\right)$, to yield a sharp crack and to minimize the size of the plastic zone ahead of the crack tip. The load and the load line displacement were monitored at a frequency of $1 \mathrm{~Hz}$. The crack mouth opening displacement (CMOD) of the $\mathrm{C}(\mathrm{T})$ specimen was measured using a sensitive linear variable differential transformer (LVDT) and the crack length was evaluated from the CMOD and load, as required by ASTM E399. 
The present experiments were carried out at the open-circuit potential in various (1 $\mathrm{M}$ to $12 \mathrm{M}) \mathrm{NaOH}$ solutions, which were prepared from reagent-grade $\mathrm{NaOH}$ and deionized water. The solution was contained in an alkali-resistant vessel fitted with a reflux condenser. The solution was not deaerated and the dissolved oxygen concentration was $\sim 8 \mathrm{ppm}$. Heating was achieved by a heating tape wrapped around the outside of the vessel. The test temperature $\left(70^{\circ} \mathrm{C}\right)$ was measured by a PTFE-coated thermocouple and was controlled to within $\pm 1^{\circ} \mathrm{C}$ using a proportional temperature controller.

SCC is a typical localized corrosion process that falls within the differential aeration (DA) hypothesis, which requires the spatial separation of the anode and the cathode for crack propagation to proceed. In a DA system with solution of high conductivity, the local anode exists in the region of the system that has the least access to the cathodic depolarizer (i.e., at the crack tip), while the local cathode(s) occurs on those regions that have the greatest access to the depolarizer (external surfaces or at least the entrance region to the crack). Thus, as the crack propagates, the electrons that are released at the crack tip flow through the metal to the external surface, where they are consumed by the reduction of the cathodic depolarizer(s) present in the solution (Figure II-2.1). Accordingly, the internal crack environment is strongly "coupled" to the external surfaces. The magnitude of the current, and hence the crack growth rate, is constrained by the conservation of charge, which is the basis of various "coupled environment" models that have been developed by this group over the past decade to describe corrosion cavity growth [II-2.5]. The (electron) coupling current was monitored using a zero resistance ammeter (ZRA) in the initial tests and using the SI1287 electrochemical interface in its ZRA mode in the later tests. This is shown schematically in Figure III-3.1. Two plate cathodes were mounted on either side of the electrically insulated, pre-cracked $\mathrm{C}(\mathrm{T})$ specimen. Tests were conducted using two sets of cathodes. Initial tests were conducted using AISI 1010 (UNS G10100) carbon steel, which has a similar chemical composition to AISI 4340 steel. The next set of tests was carried out using AISI 1018 carbon steel cathodes. Inserting the ZRA between the sample and the cathodes in this circuit permitted measurement of the coupling current produced during crack extension. Since the distance over which positive current can travel as it exits the crack mouth is limited by the 
conductivity of solution, the cathodes were placed on the sides of the $C(T)$ specimen in close proximity to the intersection of the crack plane with the surface. The potential of the specimen was also measured with respect to a platinum pseudo reference electrode, using a high impedance voltmeter in the initial tests and with respect to $\mathrm{Ag} / \mathrm{AgCl}$ reference electrode by using the SI1287 electrochemical interface in the later tests. The platinum reference electrode was placed $2 \mathrm{~mm}$ from the crack tip on one side of the specimen in the earlier tests, but in the later tests the $\mathrm{Ag} / \mathrm{AgCl}$ electrode was placed away from the crack tip and at a distance of $2 \mathrm{~mm}$ from the cathodic surface in an attempt to measure the noise in the mixed potential rather than the noise at the crack tip potential. The noise data was acquired at a frequency of $10 \mathrm{~Hz}$. A fast Fourier transform (FFT) algorithm was used to transform data from the time domain into the frequency domain, in order that periodic components in the (electron) coupling current could be detected and identified. After each fracture experiment, the fracture surfaces were examined by scanning electron microscopy (SEM).

AESmart 2000 acoustic emission apparatus fitted with a SE9125-M data transducer was used for monitoring the acoustic emission signals during crack growth. An HF/LF ratio of 1 was used. The system was configured for single channel use. The AESmart system eliminates extraneous noise sources before they enter the database, and for the first time, for any $\mathrm{AE}$ instrumentation, give information regarding the depth of a growing crack in a plate.

\section{III-3.2 Identification of the Ideal Parameters to Describe Crack Propagation}

One of the main aims of the current work was to identify the individual microfracture events in order to study their repassivation behavior, frequency of occurrence, etc., during the caustic cracking of AISI 4340 steel. Hence, it was necessary to identify the ideal conditions under which the crack propagates via individual microfracture events that can be temporally resolved.

Tests were conducted at $70^{\circ} \mathrm{C}$ at different solution concentrations (starting from $12 \mathrm{M} \mathrm{NaOH}$ down till $1 \mathrm{M} \mathrm{NaOH}$ ) and stress intensity values. Though cracking was observed giving rise to characteristic current and potential noise signals, temporal resolution of the individual microfracture events could only be realized at a solution 
concentration of $6 \mathrm{M} \mathrm{NaOH}$ and at an initial stress intensity factor of $27.4 \mathrm{MPa} \sqrt{m}$. No crack growth was observed at lower solution concentrations. The crack growth rates and the time for failure at different values of $\mathrm{NaOH}$ concentrations and initial stress intensity factor are given in Table III-3.1. Data at higher concentrations were analyzed in the frequency domain.

\section{TABLE III-3.1}

Crack growth rate under various conditions

\begin{tabular}{|c|c|c|c|}
\hline $\begin{array}{c}\text { Solution } \\
\text { concentration } \\
(M)\end{array}$ & $\begin{array}{c}\text { Initial stress } \\
\text { intensity } \\
\text { factor }(\mathrm{MPa} \sqrt{ } \mathrm{m})\end{array}$ & $\begin{array}{c}\text { Crack growth } \\
\text { rate }\left(1^{\text {st }} \text { stage }+\right. \\
\left.2^{\text {nd }} \text { stage }\right)\left(10^{-8}\right. \\
\mathrm{cm} / \mathrm{s})\end{array}$ & $\begin{array}{c}\text { Micro fracture } \\
\text { event dimension } \\
(\mu \mathrm{m})\end{array}$ \\
\hline 12 & 13 & 4700 & $\begin{array}{c}\text { Cannot be } \\
\text { resolved in time } \\
\text { domain }\end{array}$ \\
\hline 8 & 25 & 1000 & $\begin{array}{c}\text { Cannot be } \\
\text { resolved in time } \\
\text { domain }\end{array}$ \\
\hline 6 & 27.4 & 5.3 & $\begin{array}{c}49 \\
\text { No crack }\end{array}$ \\
\hline 5 & 14 & 0 & propagation \\
\hline
\end{tabular}

\section{III-3.3 Frequency Domain Analysis for High Solution Concentrations}

Tests were initially conducted at higher solution concentrations starting from 12M. The potential and the (electron) coupling current were recorded over the time period from 10 minutes before load application to final fracture, which was due to mechanical overload, as shown in Figure III-3.2. Small random fluctuations in the (electron) coupling current have been shown to be characteristic of uniform corrosion, and indeed this was the pattern that was observed before the load was applied to the specimen [Figures III-3.2(b) and III-3.2(a)]. Prior to loading, the (electron) coupling 
current was found to have a positive sign, corresponding to the flow of electrons through the metal from the cathodes to the specimen. This is most likely due to a small galvanic effect arising from the fact that the cathodes and the specimen are fabricated from slightly different materials. However, upon loading, the (electron) coupling current that flows between the specimen and the cathode first decreased to a lower value and eventually became negative, corresponding to electron flow from the specimen to the cathodes (or positive ion flow from the crack through the solution to the cathode surfaces) [Figure III3.2(b)]. Thus, during the first stage of the experiment (first 70 minutes after loading), the crack propagates at a very low growth rate, which is dictated by the existence of a residual compressive stress zone ahead of the tip of the fatigue precrack. During this period, the (electron) coupling current changes from positive to negative and the amplitude of the current fluctuations increases gradually with increasing crack length [Figures III-3.2(b) and III-3.3(b)]. During the second stage of the experiment (from 70 to 142 minutes), the crack growth rate is about $8.3 \times 10^{-5} \mathrm{~cm} / \mathrm{s}(3 \mathrm{~mm} / \mathrm{hour})$, which is significantly larger than that observed during the first stage $\left(1.7 \times 10^{-5} \mathrm{~cm} / \mathrm{s}\right)$. The (electron) coupling current and the fluctuation amplitude $(\sim 10 \mu \mathrm{A})$ remain almost constant, as shown in Figures III-3.3(c) and (d). During the final stage of fracture (after 142 minutes), the crack propagates rapidly, ultimately resulting in complete fracture by mechanical overload. The amplitude of the electron current noise increases to $20 \mu \mathrm{A}$ during the final stage [Figure III-3.3(d)], which is twice that observed during the second stage of the experiment.
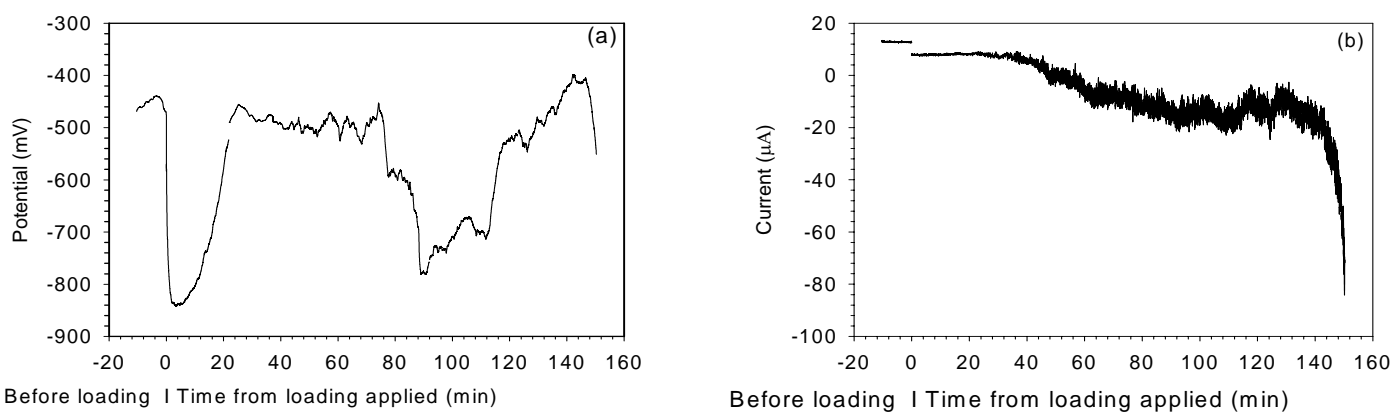

Figure III-3.2 Specimen potential (a) and (electron) coupling current (b) versus time for fracture in AISI 4340 steel in $12 \mathrm{M}$ sodium hydroxide solution at $70^{\circ} \mathrm{C}$. 
The observations summarized above confirm, in a qualitative manner, that the magnitude of the (electron) coupling current reflects the rate of crack propagation, a finding that is in concert with that of our previous work on crack propagation in sensitized Type $304 \mathrm{SS}$ in high temperature aqueous solutions [II-2.4]. The new observation from the present work is that the amplitude of the noise may also be related to the crack propagation rate.

The potential is found to shift in the negative direction promptly upon application of the load, but then recovers gradually as the load is maintained [Figure III-3.2a)]. This transition is probably related to the opening of the fatigue pre-crack, such that the freshly fractured surfaces of the fatigue precrack are exposed to the solution. The potential of the specimen then shifts in the negative direction as the growth rate increases at the beginning of the second stage, and then recovers (shifts in the positive direction) due to repassivation as the crack is opened towards final fracture due to the ever increasing stress intensity. During the final stage, both the (electron) coupling current and the potential shift sharply in the negative direction simultaneously (Figure III-3.2), because of the large fresh surface that is exposed to the solution by fast fracture due to mechanical overload. It is postulated that the potential measured here is actually the mixed potential between the $\mathrm{CT}$ specimen and the cathodes, rather than the potential at the (emergent) crack tip. This is because the reference electrode was located near the emergent crack tip on one side of specimen, but was also near a cathode that was connected to the specimen through the ZRA.The data reported here clearly demonstrate that the positive current flows through the solution from the crack tip to the external cathode during crack propagation in AISI 4340 steel in concentrated sodium hydroxide solution at $70{ }^{\circ} \mathrm{C}$. Accordingly, these data confirm that the cathodic reaction is not restricted to the flanks of the crack or to the crack mouth, but that it also occurs on the external surfaces, as postulated in the CEFM model [II-2.15]. The magnitude of the (electron) coupling current and the amplitude of the current noise increase with increasing crack growth rate. Finally, periodic pulses in the (electron) coupling current recorded during crack propagation show that caustic-cracking advances by discrete microscopic fracture events, 
as discussed below. Figure III-3.4 shows an amplitude spectrum that was obtained by transforming the (electron) coupling current from the time domain into the frequency domain by using the Fast Fourier Transform (FFT). The results indicate that the event spacing in frequency space increases while, simultaneously, the amplitude decreases with increasing frequency. This is consistent with crack advance via many small micro fracture events, characterized by small current amplitudes, occurring more-or-less simultaneously, superimposed upon less frequent, but larger discrete events of higher current amplitude. The latter events apparently occur at frequencies between 0.02 and $0.1 \mathrm{~Hz}$, as shown in Figures III-3.4 (b) and (c). The apparent transients at frequencies below $0.02 \mathrm{~Hz}$ are considered to arise from DC drift and hence may be artifacts. Before the application of the load, there are no micro fracture events, and the corresponding (electron) current amplitudes at various frequencies are very small (Figure III-3.4 (a)) and are characteristic of general corrosion.
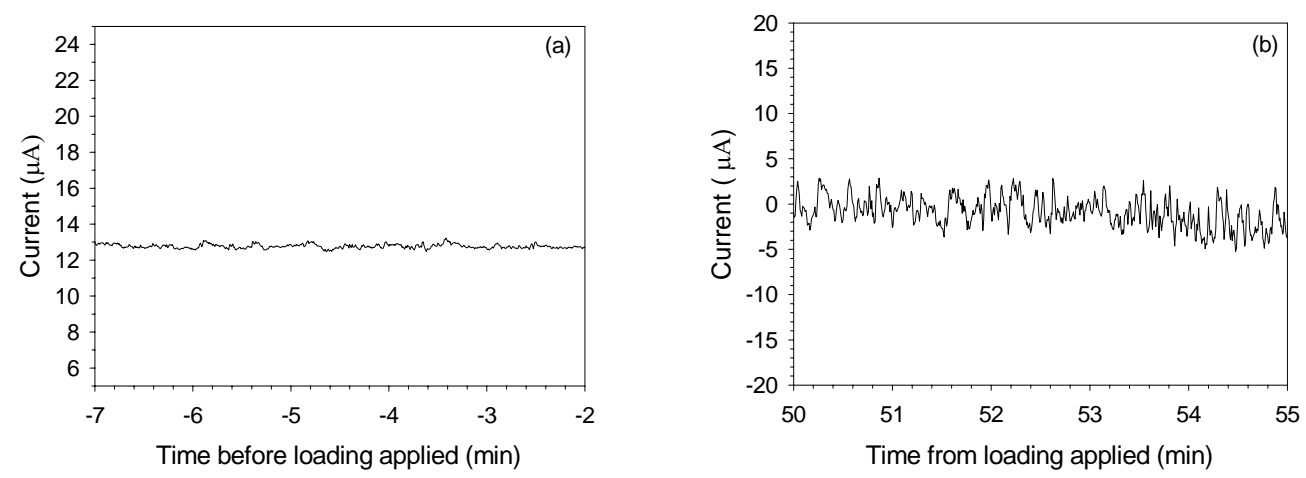

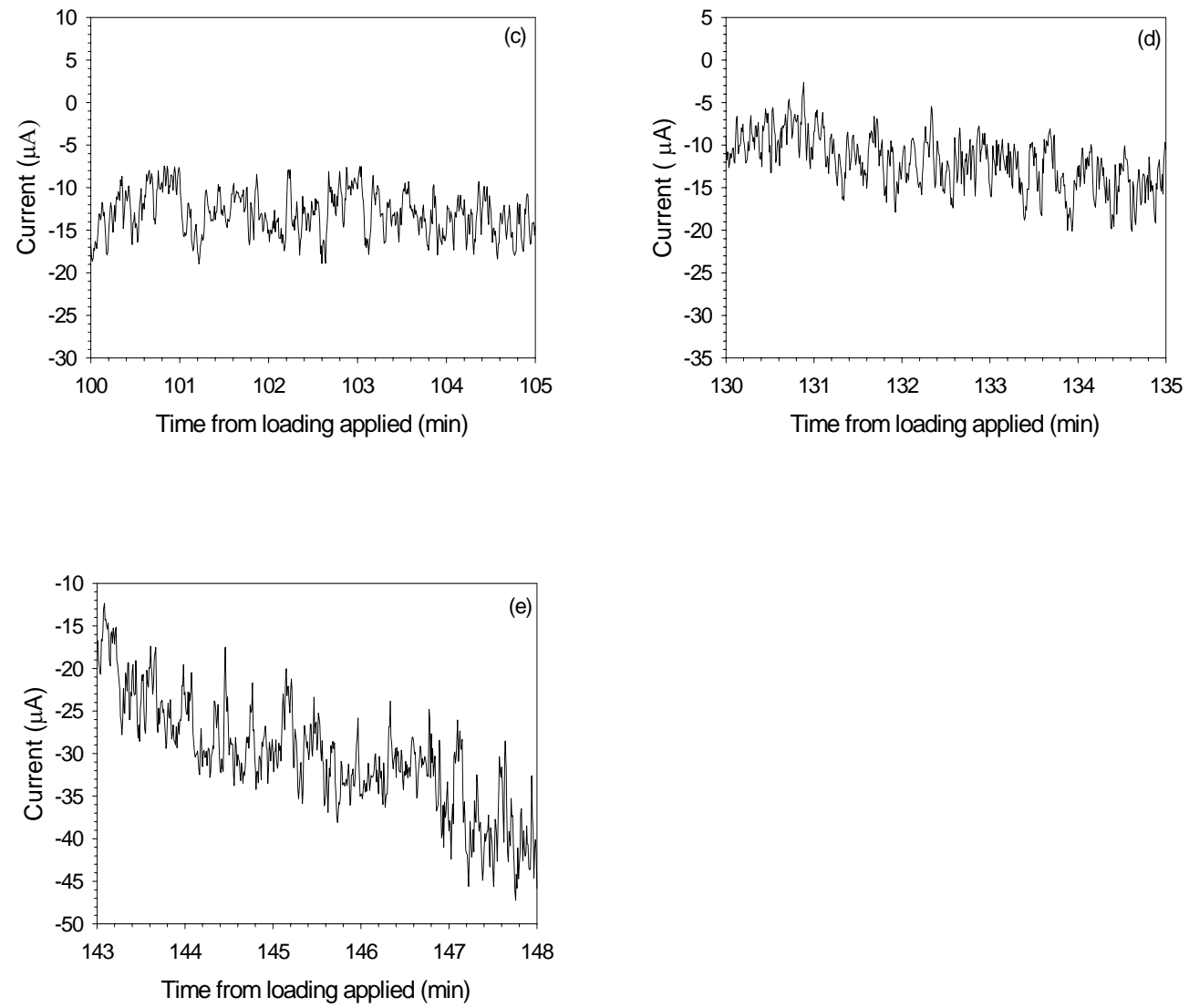

Figure III-3.3. Details of the electron current noise at various stages in the fracture history. Before loading (a) and after loading application (b, c, d and e) in $12 \mathrm{M}$ sodium hydroxide solution at $70{ }^{\circ} \mathrm{C}$.

Examination of the fracture surface by optical microscopy shows brittle morphological characteristics. The stress corrosion crack remains in the original precrack plane with no tendency for macro crack branching. Fractographic studies of the cleaned surface show that the crack propagation path in caustic solution is typical of intergranular fracture [right part in Figure III-3.5 (a)] following the transition zone, which is characterized by mixed intergranular/transgranular morphology near the front of the fatigue pre-crack [left part in Figure III-3.5 (a)]. The final fracture zone is predominantly dimpled rupture [Figure III-3.5 (b)], which is characteristic of mechanical overload. 

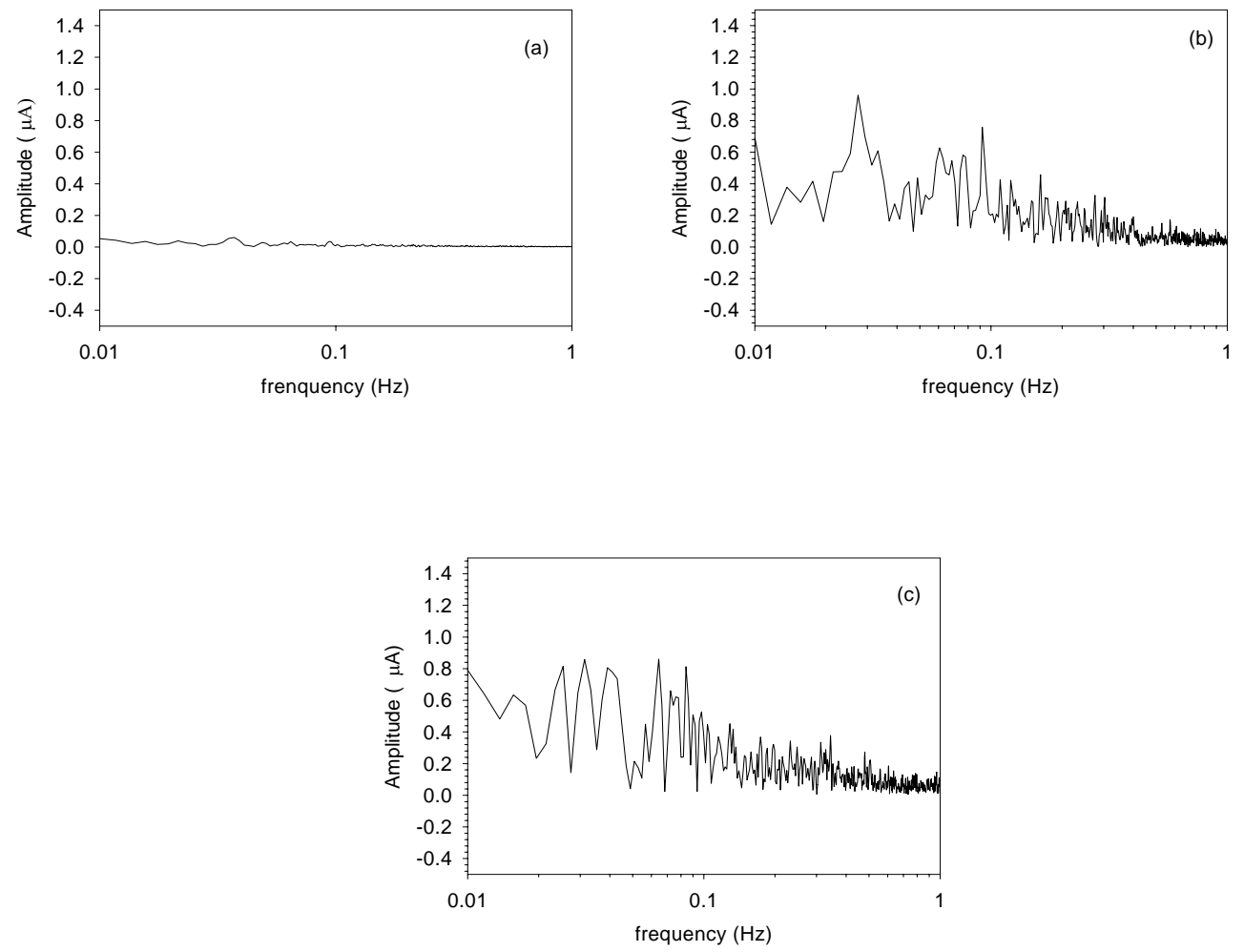

Figure III-3.4. Amplitude vs. frequency after FFT transformation of the (electron) coupling current for AISI 4340 steel in $12 \mathrm{M} \mathrm{NaOH}$ solution at $70{ }^{\circ} \mathrm{C}$. (a) Before loading application; (b) during the second stage of fracture; and (c) before final fracture of the specimen.

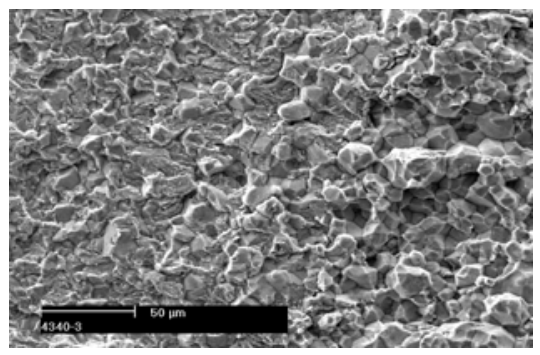

(a)

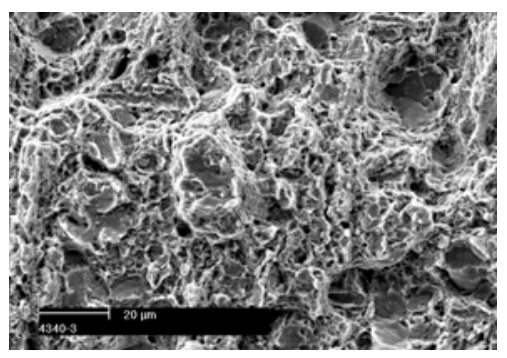

(b)

Figure III-3.5. SEM fractographs of SCC fracture surfaces produced at the free corrosion potential in $12 \mathrm{M}$ $\mathrm{NaOH}$ solution at $70{ }^{\circ} \mathrm{C}$. (a) Crack propagating from the fatigue pre-crack front (left) in mixed form to caustic cracking (right) in intergranular form. (b) The final fracture zone showing predominantly dimpled rupture (mechanical overload). 
Assuming that the frequency of the brittle micro fracture events at the crack tip decreases as the hydroxide concentration is lowered, thereby resulting in a lower crack growth rate, it must be possible to select conditions such that the fracture events are separated temporally and hence can be examined individually. Hence tests were carried out at successively lower $\mathrm{NaOH}$ concentrations. The results were similar to the results obtained at $12 \mathrm{M} \mathrm{NaOH}$ though the crack growth rate decreased with decreasing solution concentration. Individual microfracture events could be resolved only at a concentration of about $6 \mathrm{M} \mathrm{NaOH}$. The data obtained under these conditions are discussed in the next part.

\section{III-3.4 Time Domain Analysis for Lower Solution Concentrations - $6 \mathrm{M} \mathrm{NaOH}$}

In this experiment, the specimen was loaded to $998 \mathrm{~kg}$ (2200 pounds) corresponding to an initial stress intensity factor of $27.4 \mathrm{MPa} \sqrt{m}(25 \mathrm{ksi} \sqrt{\text { in }})$. Low amplitude, random fluctuations in the (electron) coupling current were observed prior to loading, as shown in Figure III-3.6, which are characteristic of uniform corrosion as stated previously. The (electron) coupling current is displaced in the negative direction promptly upon load application, but then recovers gradually to almost the same level, and has the same fluctuation characteristics, as that observed before loading. This transition is attributed to the exposure and subsequent passivation of the surfaces of the fatigue precrack to the solution upon the initial application of the load. The noise in the (electron) coupling current recorded during the subsequent 3 hours period from loading shows no obvious change, except for a small DC drift.

Four hours later, a significant transient was observed in the (electron) coupling current. Typical data collected after 4 hours from load application are shown in Figure III-3.7. In general, the (electron) coupling current contains individual, low frequency transients with amplitudes ranging from 1.5 to $7 \mu \mathrm{A}$. A typical pattern in the current noise is shown in Figure III-3.8. The transient in the (electron) coupling current is again characterized by a rapid drop and slow recovery. This type of noise in the (electron) coupling current appears to be quite characteristic of caustic SCC crack propagation. 
The kinetics of repassivation of brittle micro fracture events is an issue of considerable theoretical importance in formulating models for crack propagation. The kinetics for the case shown in Figure III-3.8 are explored in Figure III-3.9, where a first order plot of the relaxation in the coupling current is attempted. The current is indeed found to decay in a first order fashion with a rate constant of $0.029 \mathrm{~s}^{-1}$.

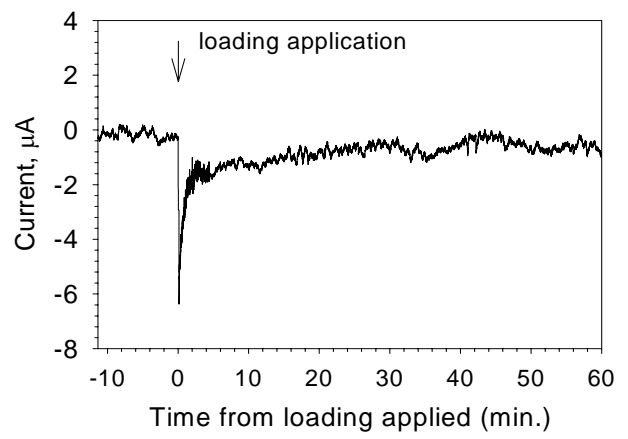

Figure III-3.6. The coupling current vs. time before and after load application in $6 \mathrm{M} \mathrm{NaOH}$ at $70{ }^{\circ} \mathrm{C}$.

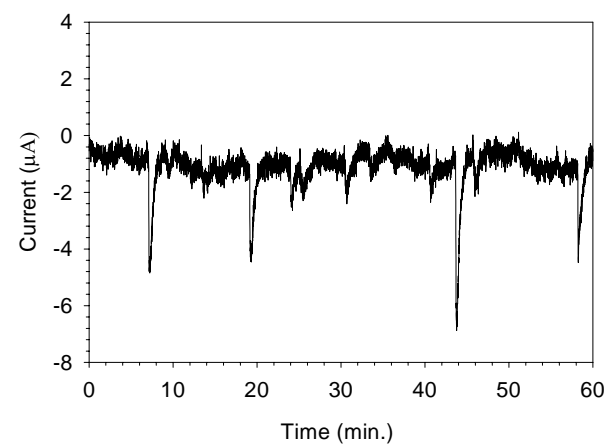

Figure III-3.7. Typical transients in the (electron) coupling current from 5 to 52 hours after load application for AISI 4340 steel in $6 \mathrm{M} \mathrm{NaOH}$ solution at $70{ }^{\circ} \mathrm{C}$. 


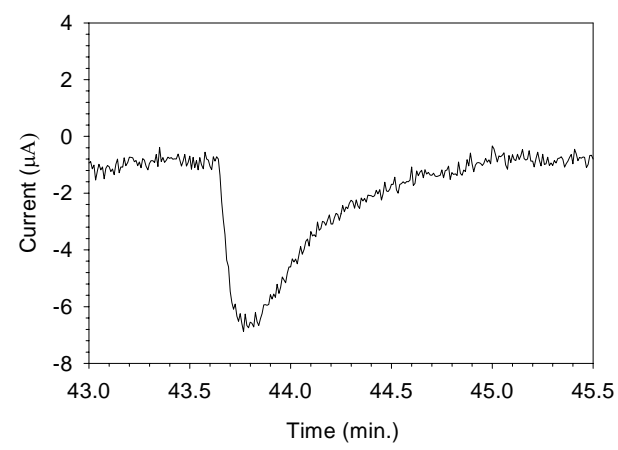

Figure III-3.8. Typical transient in the (electron) coupling current for a single fracture event in AISI 4340 steel in $6 \mathrm{M} \mathrm{NaOH}$ solution at $70{ }^{\circ} \mathrm{C}$.

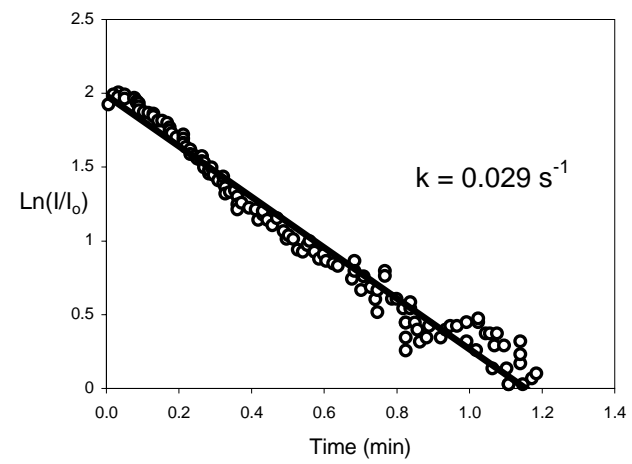

Figure III-3.9. First order kinetic plot of the repassivation current shown in Figure III-3.8

The crack propagated for about $100 \mu \mathrm{m}$ in length in intergranular form (observed by SEM) within the 52-hour observation period after loading. The average crack growth rate was $5.3 \times 10^{-8} \mathrm{~cm} / \mathrm{s}$. The results show that temporally resolved brittle micro fracture events can be observed during caustic SCC, provided that the crack growth rate is sufficiently low. Previous work [II-2.4] on the intergranular stress corrosion cracking of Type 304 SS in high temperature aqueous systems showed that the pulses in the coupling current were of a single frequency (or at least of a very narrow band in frequency) and that the fluctuations occurred in "packets" of four to thirteen fluctuations separated by brief periods of high frequency noise. Interestingly, the crack growth rate in this system was about $3 \times 10^{-7} \mathrm{~cm} / \mathrm{s}$, or about seven times higher than that observed here. In both cases, the quantity CGR/f, where CGR is the crack growth rate and $f$ is the frequency of 
transients, is similar $\left(\sim 1.5\right.$ to $\left.5 \times 10^{-7} \mathrm{~cm}\right)$, suggesting that the micro fracture dimensions are also similar. The noise signature indicates that the crack advances by one brittle micro fracture event at a time [II-2.4], probably occurring on a less-than-favorably oriented grain face with respect to the principal stress vector. Once the crack traverses the grain face and intersects a favorably oriented face (note that the crack propagates intergranularly), the crack advances rapidly, resulting in the burst of high frequency, low amplitude noise.

For AISI 4340 steel in $\mathrm{NaOH}$ solution at $70{ }^{\circ} \mathrm{C}$, the results show that the susceptibility to caustic SCC increases with $\mathrm{NaOH}$ concentration. No crack propagation is observed in $1 \mathrm{M}$ and $5 \mathrm{M} \mathrm{NaOH}$ solutions, and the noise in the (electron) coupling current comprises low amplitude, random fluctuations that are characteristic of general corrosion. Individual brittle micro fracture events are observed at low crack growth rate in $6 \mathrm{M} \mathrm{NaOH}$ solution. In more concentrated $\mathrm{NaOH}$ solution $(8 \mathrm{M}$ and $12 \mathrm{M}$ ), on the other hand, the noise in the (electron) coupling current displays periodic features that are best observed by Fourier transformation. It is postulated that caustic cracking in this high strength steel in the more concentrated caustic environments advances by many micro cracks occurring simultaneously. A transient in the coupling current signals each micro crack advance and, since many events occur at any given time, the coupling current appears as semi random fluctuations on a high mean.

Hydrogen embrittlement has been postulated to be the mechanism for environment-assisted cracking in high strength steels in caustic environments [1]. Substantial evidence exists in the literature that the chemical environment at the tip of a stress corrosion crack can differ markedly from the bulk solution composition, due to the "occluded cell" (differential aeration) effect. Brown et al. [2, 3] found that the solution at the tip of a crack in AISI 4340 steel in chloride containing solution always had a $\mathrm{pH}$ of 3.5 to 3.9, even though the bulk pH varied between 2 and 10. The establishment, in that case, of a steady state $\mathrm{pH}$ at the tip is due to hydrolysis equilibria involving the soluble corrosion products (e.g., $\mathrm{Fe}^{2+}$ ).

The potential at the crack tip for high-strength steel always lies at or below the "hydrogen line" for the prevailing crack tip $\mathrm{pH}$, for a variety of external conditions. 
Thus, the observations of this work can be explained by a brittle micro fracture mechanism, in which the events are possibly induced by hydrogen. We postulate that the large current exiting the crack mouth, which has been detected by the ZRA measurements reported here, generates a lower $\mathrm{pH}$ at the crack tip and that hydrogen is injected into the local metal matrix ahead of the crack tip. Hydrogen atoms then diffuse to the grain boundaries where embrittlement occurs by pressurization of micro voids or by chemical decohesion via reaction with segregated metalloids (e.g., $\mathrm{P}, \mathrm{Si}, \mathrm{N}$ ) [4]. In any event, the periodic fracture events are initiated at points ahead of the crack at which the hydrostatic stress and the hydrogen concentration exceed critical conditions. This mechanism is proposed, in spite of the fact that the environment external to the crack is highly alkaline and oxidizing. Furthermore, we note that the fractographic characteristics of caustic cracking in AISI 4340 steel in $\mathrm{NaOH}$ solution, as observed in this work, are very similar as those found for hydrogen embrittlement in precracked specimens of the same steel in gaseous hydrogen [5], an environment in which anodic dissolution is not possible.

Finally, assuming that the fracture events are semi-circular in geometry of radius $r$ and that the frequency at which they occur is $f$, the crack growth rate can be written as

$$
\frac{d L}{d t}=\frac{2 r^{2} f}{B_{n}}
$$

where $B_{n}$ is the specimen thickness at the groove. Equation (1) is readily rearranged to yield the micro fracture dimension as

$$
r=\sqrt{\frac{B_{n} \cdot d L / d t}{2 f}}
$$

For the $6 \mathrm{M} \mathrm{NaOH}$ case, $d L / d t=5.3 \times 10^{-8} \mathrm{~cm} / \mathrm{s}, B_{n}=2.7 \mathrm{~cm}$, and $f \approx 0.003 \mathrm{~s}^{-1}$ (Figure III-3.7), yielding a micro fracture dimension of $\approx 49 \mu \mathrm{m}$. Note that the fracture frequency is somewhat subjective, because it depends upon what is counted as a "fracture event". In the present case, eleven events were counted over the sixty minutes recorded 
in Figure III-3.7, resulting in the value given above for $f$. Acoustic emission test results in the second heat of samples confirm the assumption that the individual transients indeed represent individual crack events in most of the cases. The calculated fracture dimension may be compared with the $3 \mu \mathrm{m}$ dimension found for the fracture of sensitized Type 304 $\mathrm{SS}$ in high temperature water [II-2.2]. It is likely, however, that larger micro fracture events give rise to the more intense current transients, so that the $49 \mu \mathrm{m}$ dimension estimated above probably represents the upper end of a distribution in this quantity. Because the dimension of a slip event should be a small multiple of the Burger's vector (nanometers), the fracture dimension appears to be much too large to be consistent with the slip/dissolution/repassivation mechanism for crack advance. On the other hand, the dimension is consistent with a hydrogen embrittlement mechanism, noting that the fracture dimension is expected to correspond with the spacing of some metallurgical asperity (e.g., precipitates) on the grain boundary or with the grain size itself (note that the grain size as revealed on the fracture surface is $10-40 \mu \mathrm{m}$, Figure III-3.5).

\section{III-3.5 Anisotropy of Stress Corrosion Cracking in AISI 4340 Steel}

The results discussed in sections III-3.2 through III-3.4 were for the specimens that were prepared in the short transverse-longitudinal $(\mathrm{S}-\mathrm{L})$ orientation. The results that will be discussed in this section will be for the specimens that were prepared in the longitudinal-transverse (L-T) orientation.

Figure III-3.10 shows the orientation of test specimens [6].

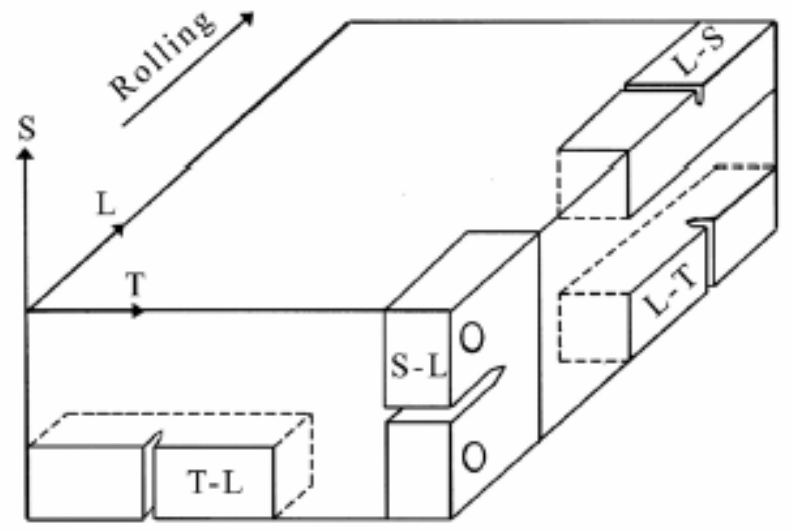

Figure III-3.10. Rolling direction and different specimen orientations 
The same tests that were conducted on the specimens with S-L orientation were conducted on the specimens with L-T orientation. In this case crack extension was monitored simultaneously using AET. Though the tests conducted under these conditions yielded similar transients, some striking differences were noted. These differences are summarized below and discussed subsequently.

1. Crack growth rate decreased drastically in the case of L-T orientation specimens compared to S-L orientation specimens.

2. The kinetics of repassivation changed significantly with the specimen orientation

3. The value of $\mathrm{K}_{\mathrm{ISCC}}$ under identical solution concentrations was higher in the case of L-T orientation specimens.

These results have important implications on the crack growth rate mechanisms which will be discussed later in the section. The experimental results for these tests are given below.

For L-T orientation specimens, low amplitude, random fluctuations in the (electron) coupling current, as observed in the case with S-L specimens, were observed prior to loading, as shown in Figure III-3.11. The (electron) coupling current is displaced in the negative direction promptly upon load application, but then recovered gradually, and had similar fluctuation characteristics, as that observed before loading. The noise in the (electron) coupling current recorded during the subsequent 5 hour period from loading showed no obvious change, except for a small DC drift. There was no crack growth in the first five hours. This has been confirmed from the acoustic emission data (Figure III-3.12). The first transient which appears at the end of four hours (at around 17000 seconds in figure III-3.13) coincides with the first significant spike in the acoustic emission counts. An hour of data acquired after four hours of load application is shown in Figure III-3.13. 


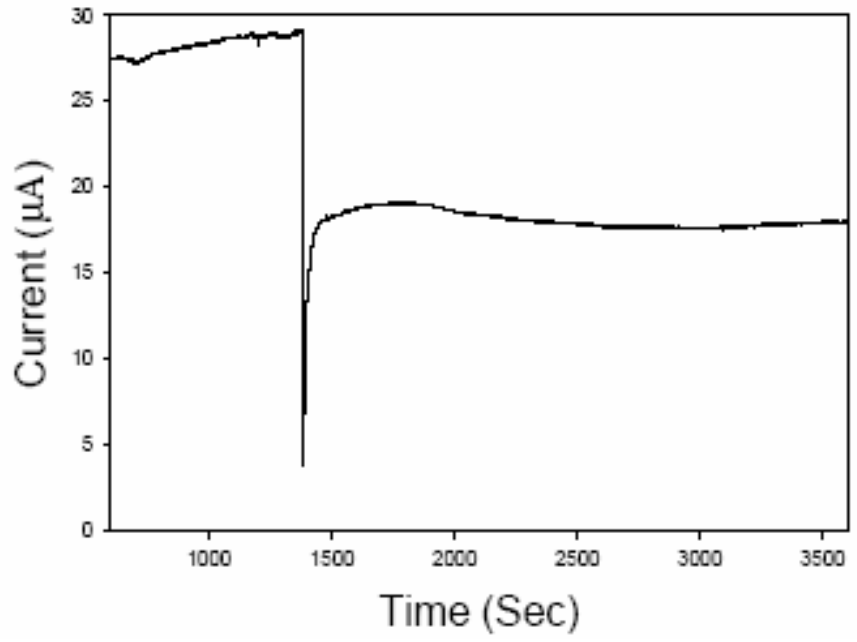

Figure III-3.11. Electron coupling current vs. time before and after load application in $6 \mathrm{M} \mathrm{NaOH}$ at $70{ }^{\circ} \mathrm{C}$ for the L-T orientation sample.

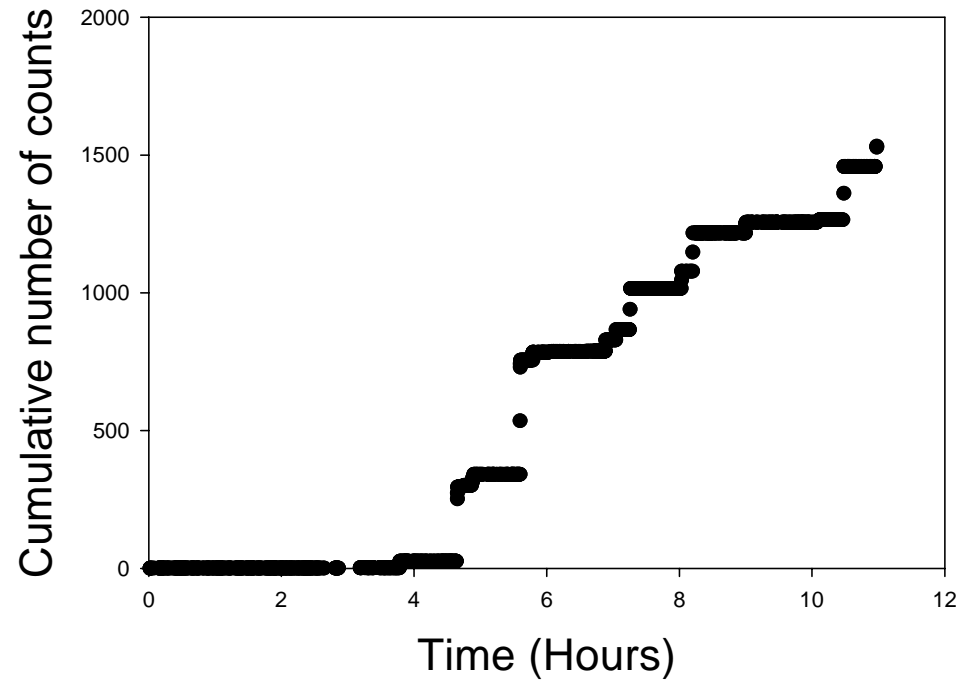

Figure III-3.12. Cumulative acoustic emission counts vs. time after the application of load for the L-T orientation sample 


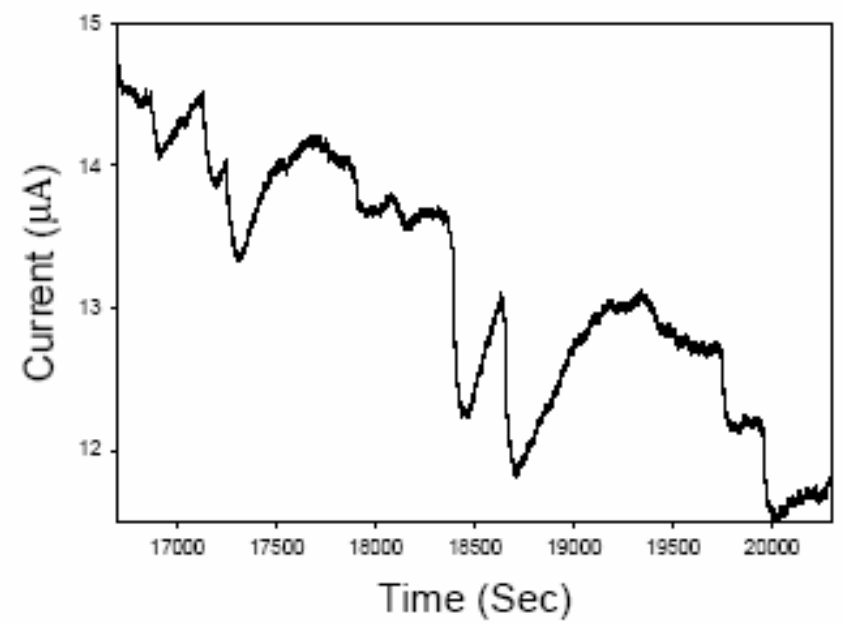

Figure III-3.13. Typical transients in the (electron) coupling current 4 hours after load application for AISI 4340 steel in $6 \mathrm{M} \mathrm{NaOH}$ solution at $70{ }^{\circ} \mathrm{C}$ for the L-T orientation sample

Notice that the repassivation times of these samples are significantly longer than the S-L orientation specimen times (Figure III-3.14). We can also notice that the repassivation time is greater than the time of separation between two crack events in most of the cases. This leads to accumulation of coupling current. Only very few crack events that were observed over a period of six hours underwent complete repassivation. This was not the case with the S-L orientation specimens (Figure III-3.7).

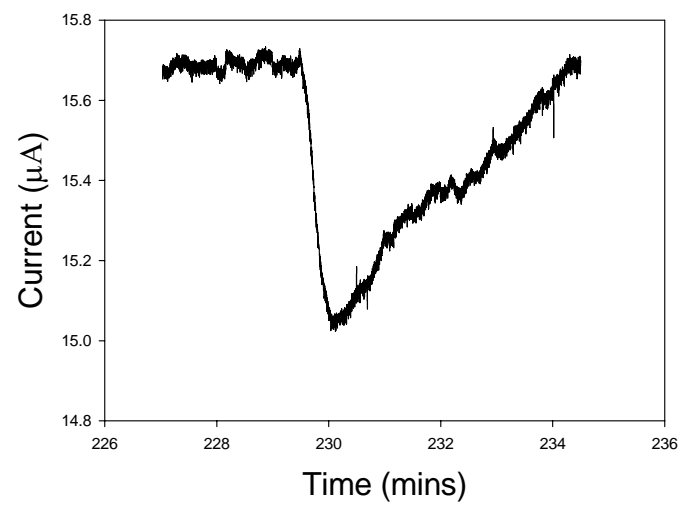

Figure III-3.14. Typical transient in the (electron) coupling current for a single fracture event in AISI 4340 steel in $6 \mathrm{M} \mathrm{NaOH}$ solution at $70{ }^{\circ} \mathrm{C}$ for the L-T orientation sample

The current in this case is still found to decay in a first order fashion, as before, but at a much slower rate as indicated by the rate constant of $0.00026 \mathrm{~s}^{-1}$ (Figure III- 
3.15). Recall that the rate constant in the previous case was calculated to be $0.026 \mathrm{~s}^{-1}$, which is more than an order of magnitude larger than this.

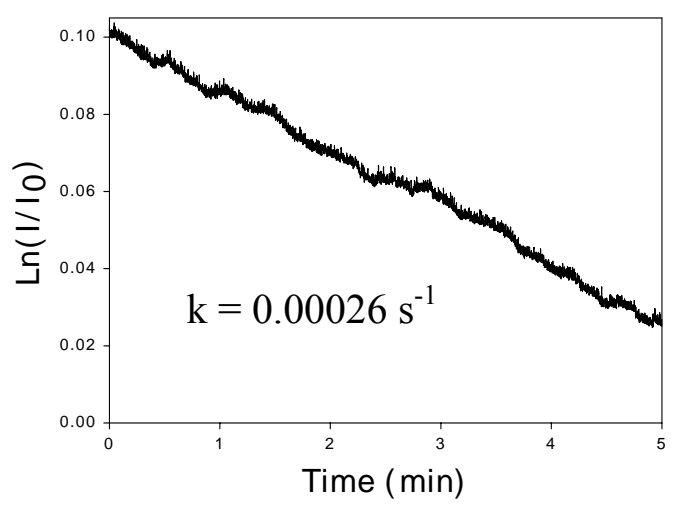

Figure III-3.15. First order kinetic plot of the repassivation current shown in Figure III-3.14

The acoustic emission event rate is commensurate with the current noise event frequency. For example, the first transient in the coupling current (Figure III-3.14) which occurs roughly 230 minutes after the application of load coincides with the first significant increase in the AE counts (Figure III-3.16). In the subsequent 6 hours of data acquisition, the coupling current transients coincided with the $\mathrm{AE}$ counts in most cases. This indicates that the individual transients in the coupling current were indeed due to the individual microfracture events as postulated.

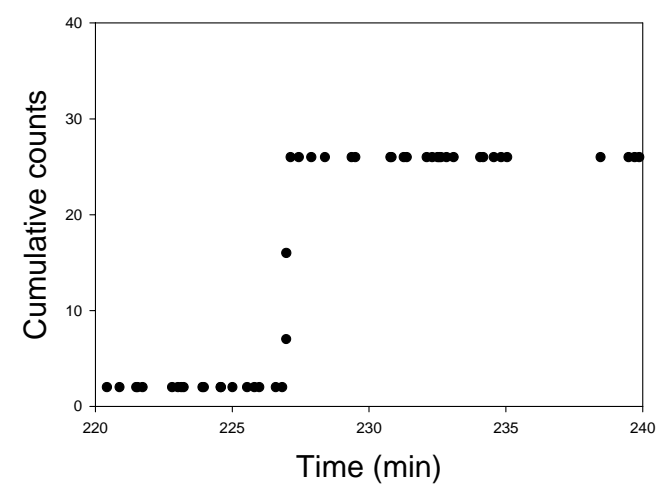

Figure III-3.16. First spike in the acoustic emission count corresponding to the first crack event

The Crack growth rate was negligible in the case of L-T orientation specimens in $6 \mathrm{M}$ sodium hydroxide solution and it couldn't be detected using the LVDT. $\mathrm{K}_{\text {ISCC }}$ in the 
case of L-T orientation specimens in $12 \mathrm{M} \mathrm{NaOH}$ was about $30 \mathrm{ksi}$ in when compared to $12 \mathrm{ksi}$ in in the case of S-L orientation specimens under identical conditions.

This decrease in crack growth rate and increase in $\mathrm{K}_{\mathrm{ISCC}}$ in the case of L-T orientation specimens is due to the L-T orientation specimens possessing higher fracture toughness in comparison to the S-L specimens [6-7]. Figure III-3.17 shows a comparison of the fracture stress for micro alloyed line pipe steel for different specimen orientations [6].

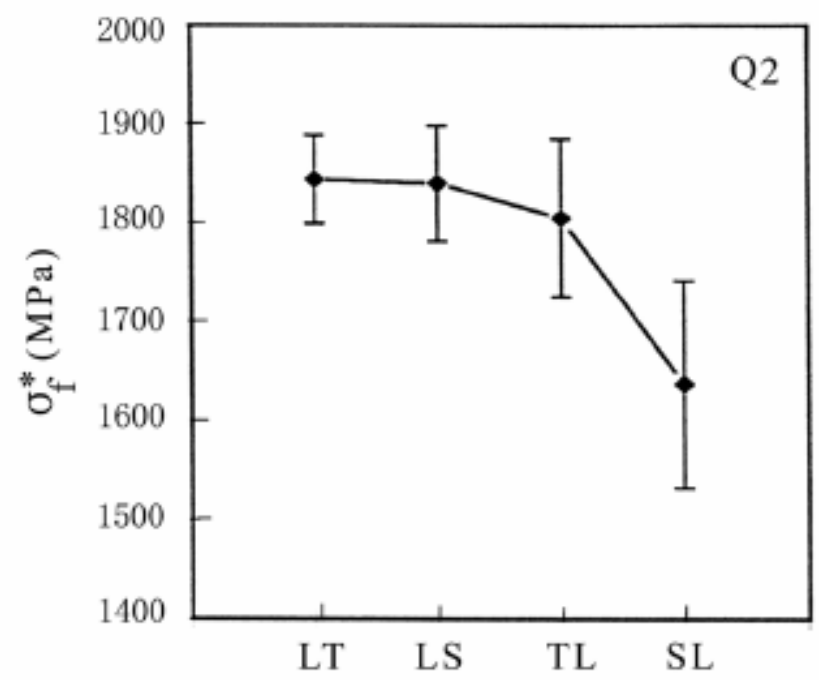

Figure III-3.17. Variation of fracture stress with specimen orientation in micro alloyed line pipe steels

We can see that that the fracture stress is the highest for the L-T orientation and is the lowest for the S-L orientation. This result explains the higher $\mathrm{K}_{\mathrm{ISCC}}$ and lower crack growth rates experienced in the case of L-T orientation specimens in the AISI 4340 steel in $\mathrm{NaOH}$ solutions.

\section{III-3.6 Role of Crack Flanks in the Stress Corrosion Cracking of AISI 4340 Steel in $\mathrm{NaOH}$ Solutions}

The role of crack flanks in sustaining the cathodic reaction that is coupled to the anodic reaction occurring at the crack tip has always been a contentious issue among the stress corrosion cracking community [7-8] The results of the tests conducted using AISI 4340 steel in $\mathrm{NaOH}$ solutions of different concentrations give a clear picture of the role of crack flanks in sustaining the coupled cathodic reaction. 
Figure III-3.18 shows the cross section of an AISI 4340 compact tension specimen that was fractured in one of the SCC tests. We can clearly see that, unlike the fatigue pre-crack's (a mechanical crack) crack front, which is uniform throughout the thickness of the $\mathrm{C}(\mathrm{T})$ specimen, the crack front of the stress corrosion crack is concave (Crack grows predominantly along the sides). This is because of the electrochemical nature of the stress corrosion cracks. The sides, which have a high proximity to the external cathodes, grow extensively in comparison to the centre of the specimen, which is relatively far off from the external cathodes. The CGR along the sides was found to about 7-20 times the CGR along the centre of the specimen.

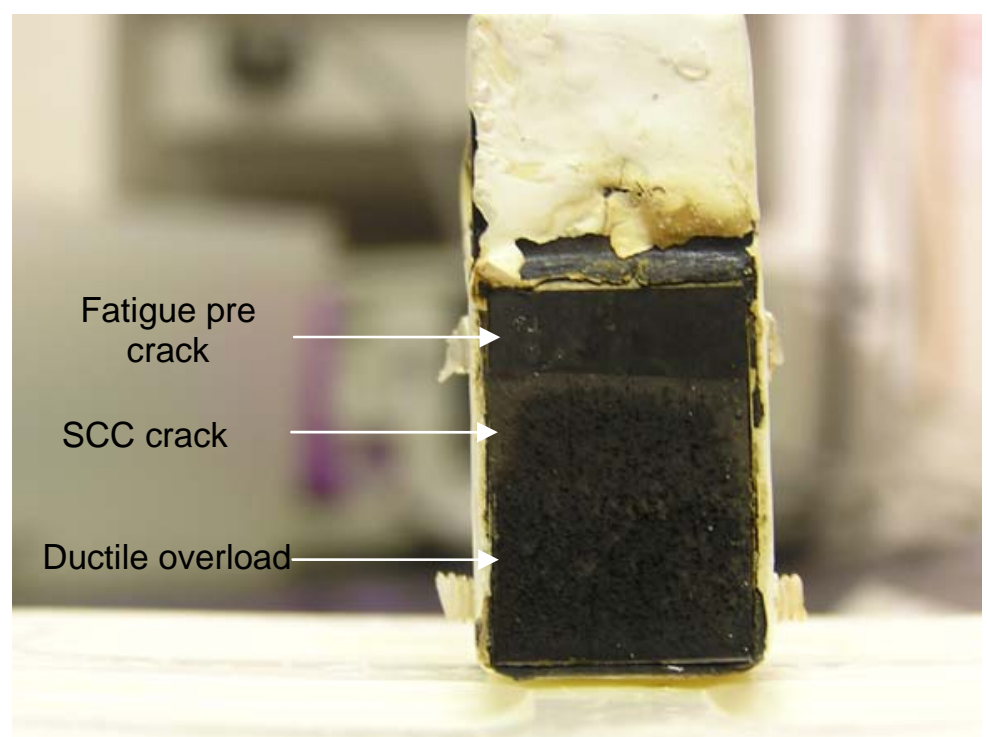

Figure III-3.18. Concave shaped crack front indicating the propagation of the crack predominantly along the sides

These results clearly prove that it is the external cathodes and not the crack flanks that sustain bulk of the cathodic reaction. This has already been proved by Zhou, Balachov and Macdonald [9]. In their experiment, CGRs of type 304SS compact tension specimens, with and without a $\mathrm{ZrO}_{2}$ dielectric coating, were measured under a high temperature sodium sulfate environment. The result is shown in the figure below. 


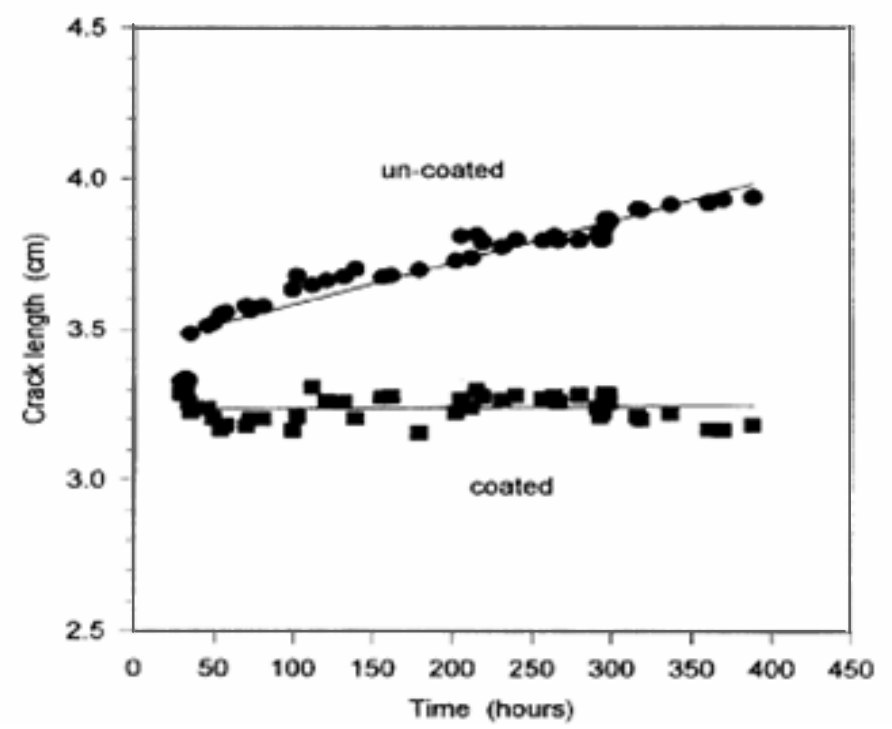

Figure III-3.19. Crack length vs. time for a coated C(T) specimen and an uncoated C(T) specimen of sensitized Type $304 \mathrm{SS}$ in high temperature water

The CGR of the coated specimen, for which the crack flanks were the only uncoated part (apart from the crack tip), was found to be more than 10 times less than the CGR of the uncoated specimen. This is a clear indication of the fact that, it is the external cathodes and not the crack flanks that sustain the major part of the cathodic reaction.

A similar test was carried out in the lab to test the role of crack flanks in sustaining the stress corrosion crack. SCC tests were carried out in a teflon coated AISI 4340 sample. An external cathode, made of the same material, was attached to only one side of the specimen. This was done, on purpose, to check if the concave crack front was actually an effect of the coupled cathodic reactions occuring external cathodes. Figure III-3.20 shows the cross section of the fractured sample. We can see that the stress corrosion crack has grown extensively on the left hand side, along which the only cathode was attached.

This is again a clear proof of the fact that it is the external cathodes that sustain the major part of the cathodic reaction. In other words there is not enough differential aeration between the crack tip and the crack flanks to generate enough driving force to sustain the crack. 


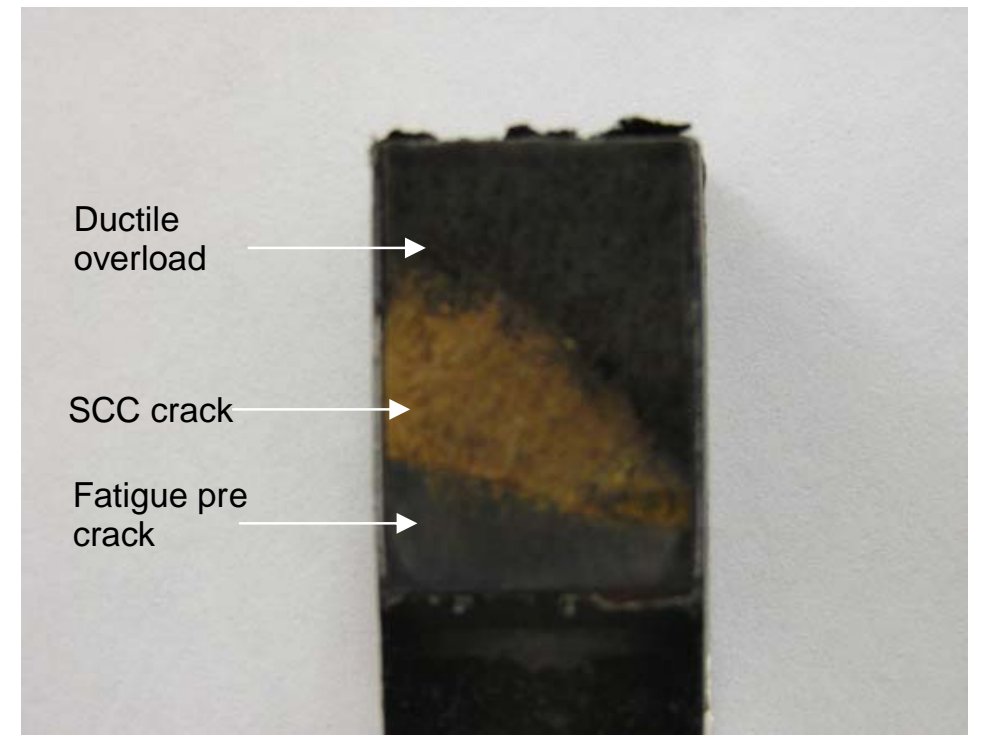

Figure III-3.20. Fractured CT specimen with external cathode loaded only on one side of the C(T) specimen

\section{III-3.7 Role of Crevice - Darwinian Death of a Stress Corrosion Crack in the Presence of a Crevice}

The teflon coating at the side grooves of the compact tension specimens was scraped off to expose the grooves to the sodium hydroxide solution, as explained in the experimental section. At high temperatures $\left(70^{\circ} \mathrm{C}\right)$ and solution concentrations $(6 \mathrm{M}-12$ $\mathrm{M})$, the coating begins to delaminate both above and below the side grooves of the specimen. This leads to the formation of crevices at the delaminated areas. This crevice formation has a profound effect on the crack propagation as explained below.

Figure III-3.21 shows the fluctuations in coupling current in a system with a crevice created by de-lamination, at $70^{\circ} \mathrm{C}$. 


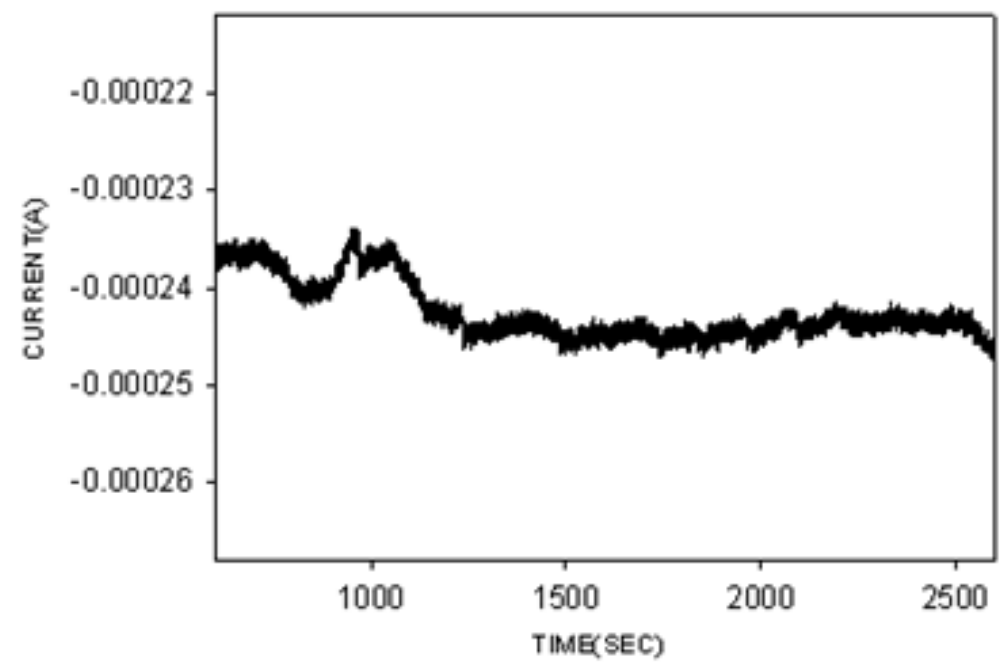

Figure III-3.21. Fluctuations in coupling current in a AISI $4340 \mathrm{C}(\mathrm{T})$ specimen with a crevice created by de-lamination, at $70^{\circ} \mathrm{C}$ in $8 \mathrm{M} \mathrm{NaOH}$ solution

Notice that the value of the coupling current is significantly greater than expected. This current is due to the presence of a crevice above and below the side grooves on either side of the compact tension specimen. This is supported by figure III-3.22 which shows that the magnitude of the coupling current relaxes from $380 \mu \mathrm{A}$ to about $0 \mu \mathrm{A}$ when the temperature is decreased from $70^{\circ} \mathrm{C}$ to room temperature.

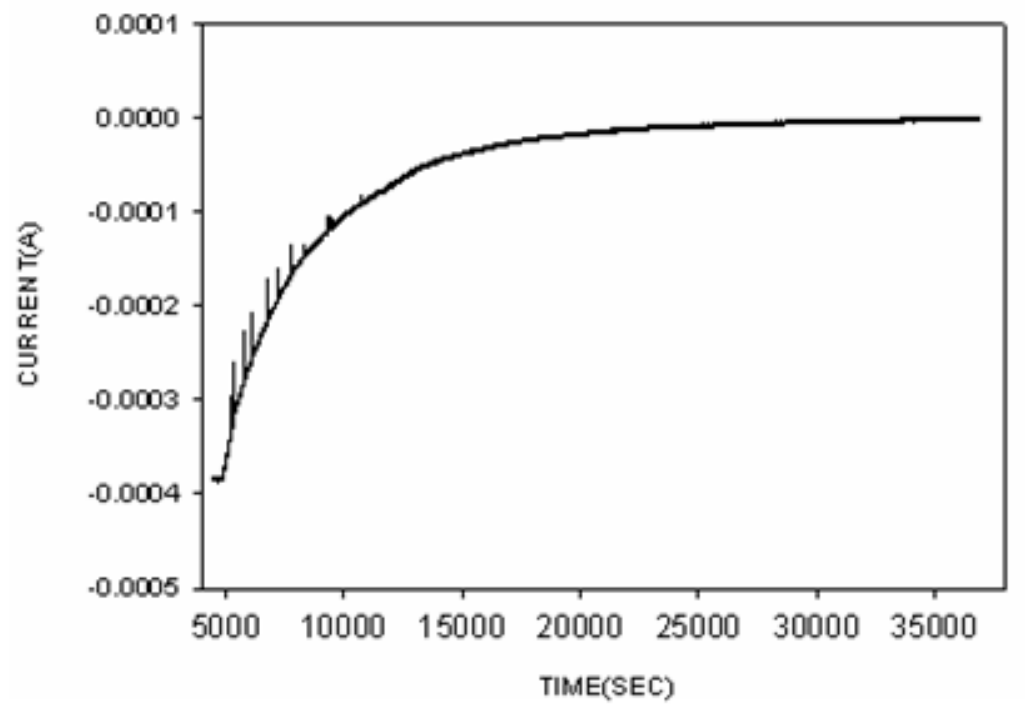

Figure III-3.22. Relaxation of the coupling current from $380 \mu \mathrm{A}$ to $0 \mu \mathrm{A}$ when the temperature is decreased from $70{ }^{\circ} \mathrm{C}$ to room temperature. This is typical of a system with a crevice.

This type of current relaxation is typical of a crevice current as shown in the figure given below. This figure shows the crevice current as a function of temperature in a titanium alloy in $\mathrm{NaCl}$ solution [10]. 


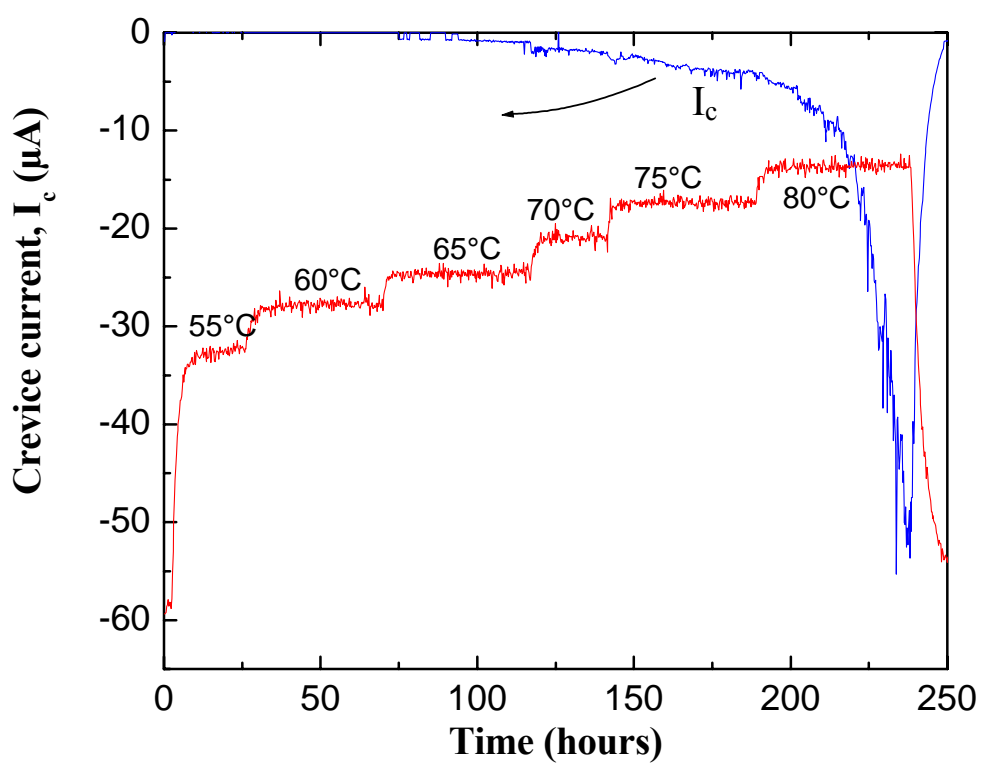

Figure III-3.22. Crevice current in Ti-2 alloy in $\mathrm{NaCl}$ solution as the temperature was increased in steps and then decreased

We can see that after the crevice initiates at about $65{ }^{\circ} \mathrm{C}$, the crevice current increases with temperature and relaxes back to about $0 \mu \mathrm{A}$ when the temperature is decreased.

Figure III-3.23 shows the potential fluctuations in AISI 4340 steel in $\mathrm{NaOH}$ solution, with and without the presence of crevice at $70^{\circ} \mathrm{C}$.

Notice that the potential of the sample has shifted from -1.1 V vs. SCE, in the case of the specimen without crevice, to about $600 \mathrm{mV}$, in the case of the specimen with crevice. The presence of a crevice polarizes the system.

Such potential shifts are very common in crevice corrosion systems as shown in the figure below [11]. Figure III-3.24 shows how the onset of crevice at $65^{\circ} \mathrm{C}$ shifts the potential of the titanium alloy in the negative direction, thereby significantly polarizing it. 


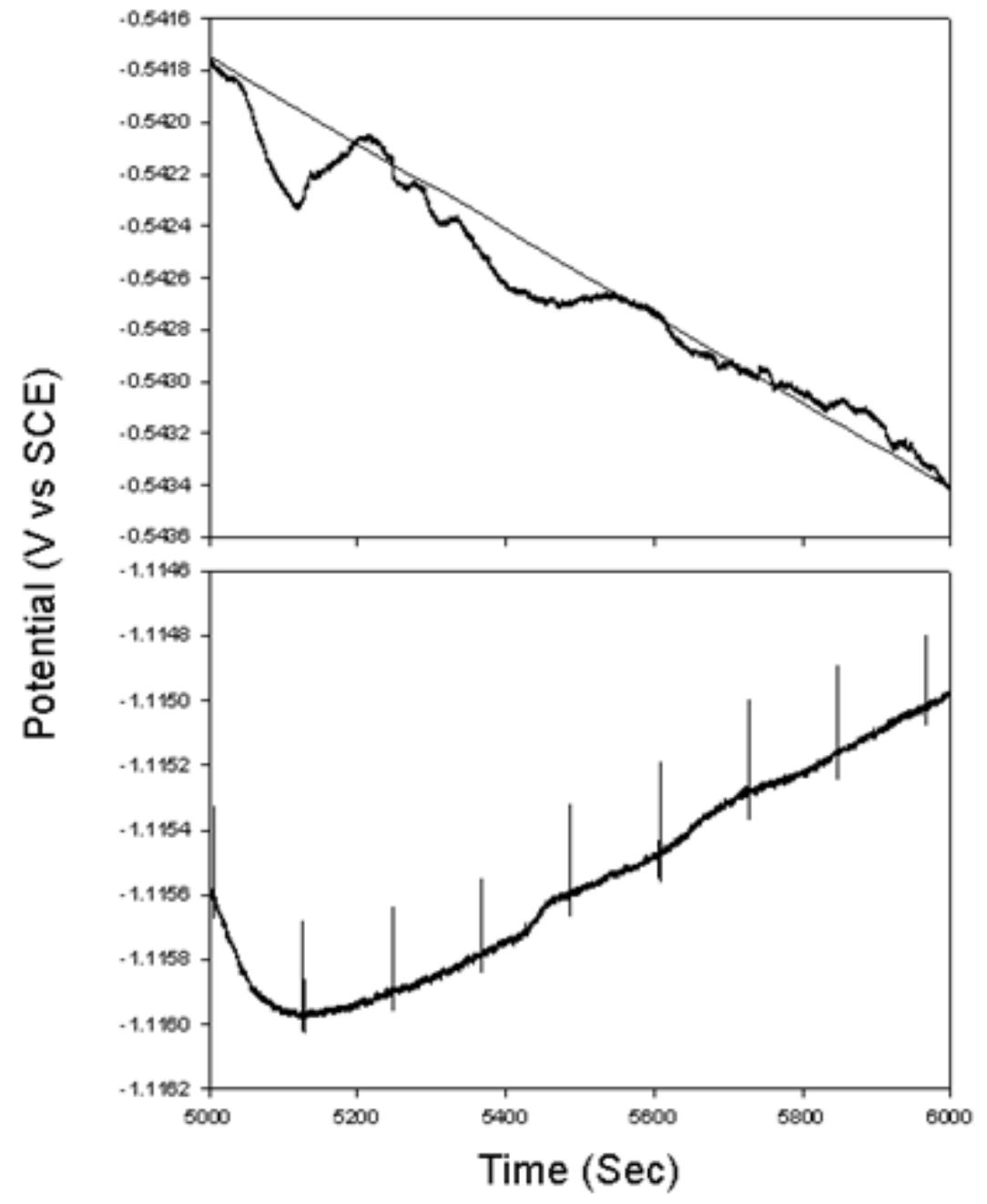

Figure III-3.23. Potential fluctuations in AISI 4340 steel in $\mathrm{NaOH}$ solution. a) With and b) without the presence of crevice at $70{ }^{\circ} \mathrm{C}$ 


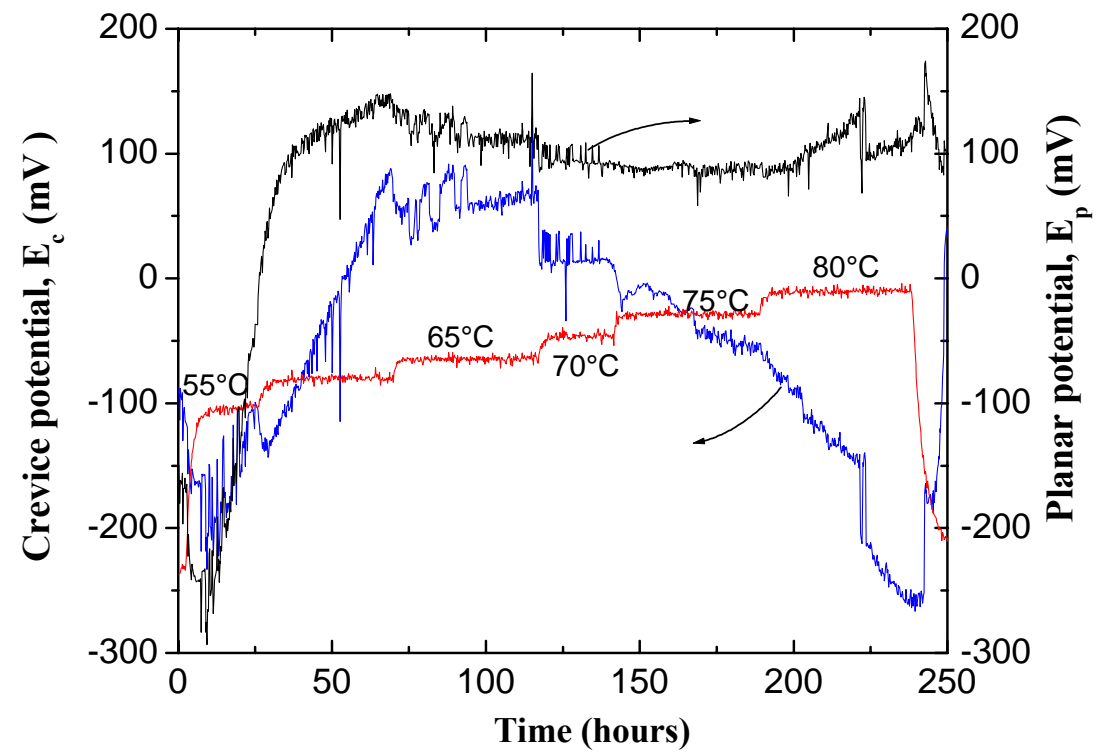

Figure III-3.24. Crevice potential in $\mathrm{Ti}-2$ alloy in $\mathrm{NaCl}$ solution as the temperature was increased in steps and then decreased

A postmortem of different samples revealed mild to extensive crevice growth under various experimental conditions. This is shown in figure III-3.25 through figure III3.27. Figure III-3.25 shows a case of extensive crevice growth, while figure III-3.26 shows a case of mild crevice growth.

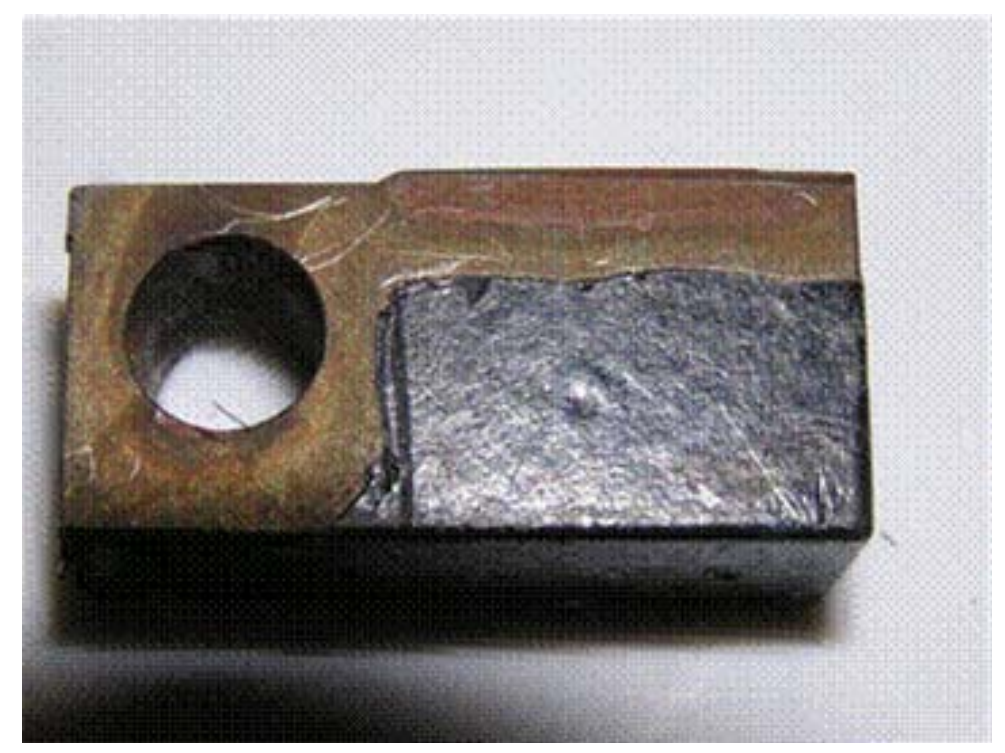

Figure III-3.25. A case of extensive crack growth along the delaminated region below the side groove 


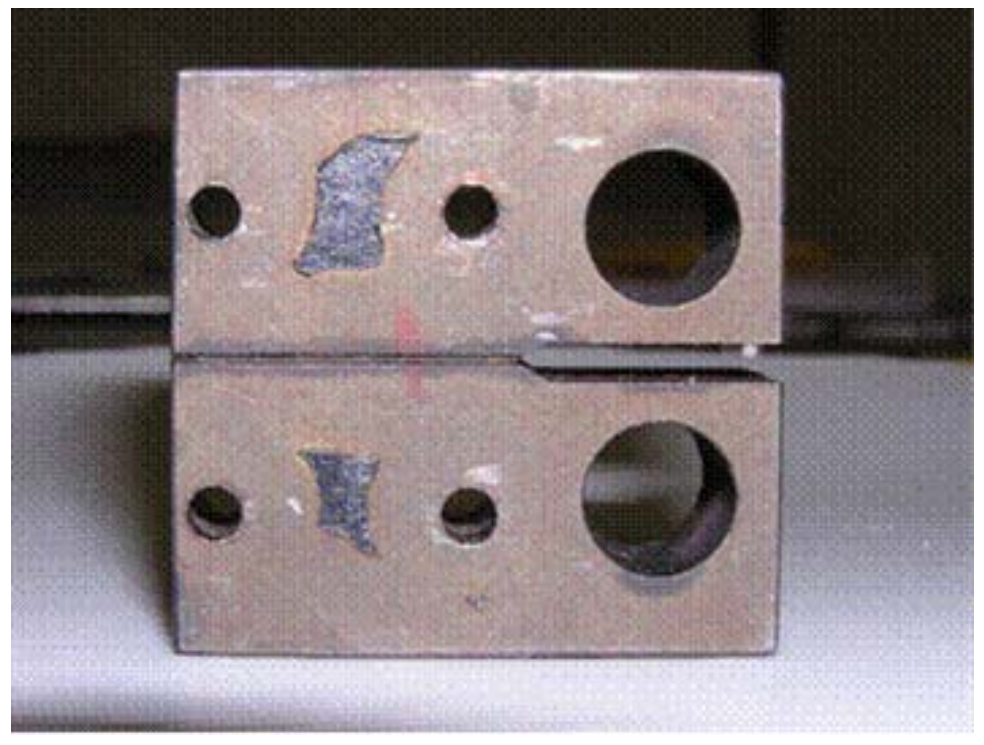

Figure III-3.26. A case of mild crack growth along the delaminated regions above and below the side groove

Apart from regions above and below the side grooves, regions in the periphery of the holes, which were drilled to fasten the external cathodes, also underwent extensive crevice corrosion. This is shown in the figure below.

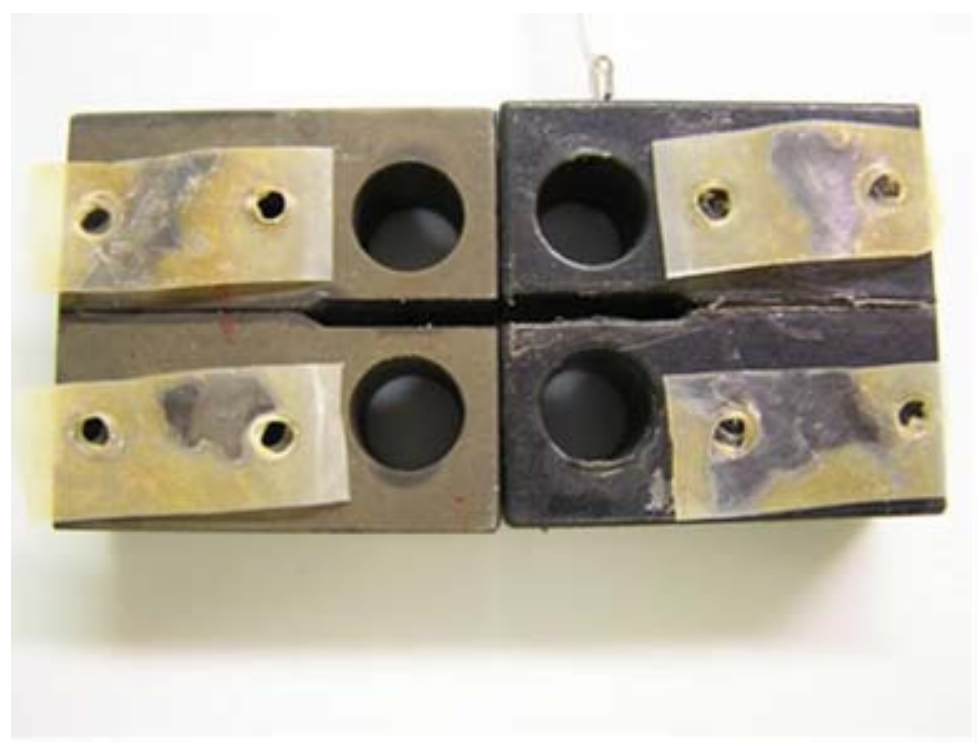

Figure III-3.26. Extensive crevice corrosion, due to delamination, in the periphery of the holes that were drilled to fasten the external cathodes 


\section{Crack growth rate}

The crack growth rate (CGR) of the samples that had the problem of crevice corrosion was negligible in comparison to the samples that didn't have the problem (CGR of a sample with no crevice was about $0.5 \mathrm{~cm} /$ day and that of a specimen with crevice was about $0.1 \mathrm{~mm} /$ day at $70^{\circ} \mathrm{C}$ in $8 \mathrm{M} \mathrm{NaOH}$ solution). The reason for this drastic decrease in crack growth rate in the case of samples with crevice corrosion can be explained as follows.

Lenhart, English and Macdonald [12], while investigating the phenomenon of nucleation and growth of pits in buffered chloride solution by using in situ optical microscopy, made the following two observations.

i) Each pit that nucleated on the surface and grew had associated with it a hemisphere of influence (HOI) which is produced by a high concentration of hydrolyzed metal ions. No new, stable pits nucleated under the HOI of an existing pit.

ii) The overlaps of HOIs from neighboring pits inevitably lead to the death of one of the pits.

The first observation was attributed to the fact that a pit cathodically protects the external surface under the HOI, due to the preponderance of the cathodic partial current on the surface. The second observation was attributed to the competition between pits for the available resources on the external surface. The fittest survives leading to the death of the unfit pit.

Just like pitting, crevice and stress corrosion phenomena also come under the category of localized corrosion phenomena. Hence, a crevice or a stress corrosion crack could have their own HOIs. Since the size of the crevice is much larger than the size of the stress corrosion crack, and since the crevice and crack are in close proximity to each other, the crack could come under the HOI of the crevice and hence get cathodically polarized. This would decrease the crack growth rate.

The HOIs of the crack and the crevice could also overlap leading to the Darwinian death of the weak stress corrosion crack.

These two reasons would collectively lead to the drastic decrease in CGR of the stress corrosion crack. 


\section{References III-3:}

1. R. C. Newman and R. P. M. Procter, British Corrosion Journal, 25, 259 (1990).

2. B. F. Brown, ed., Stress Corrosion Cracking in High Strength Steels and in Titanium and Aluminum Alloys, Naval Research Laboratory, 1972, p.80-145

3. B. F. Brown, Stress Corrosion Cracking and Hydrogen Embrittlement of Iron Base Alloys, Unieux-Firminy, France, June 12-16, 1973, NACE International, USA, p.747-750

4. B. Craig, ASM Handbook, ASM International, 13, Corrosion, 163 (1990)

5. D. Hardie and S. Liu, Corros. Sci., 38, 721 (1996).

6. Jun Sun and J.D. Boyd, International Journal of Pressure Vessels and Piping, 77, 369 (2000)

7. Soundararajan, B. and Macdonald, D. D., "Caustic Cracking of AISI 4340 Steel", Paper \#04572, CORROSION '04, New Orleans, LA, March 2004.

8. P.L Andresen, T.P Diaz, and S Hettiarachchi, $11^{\text {th }}$ International Conference on Environmental Degradation of Materials in Nuclear Systems, 512, (2003)

9. X Zhou, I Balachov, and D.D Macdonald, Corrosion Science, 40, 1349 (1998)

10. K.T venkateswara Rao, W Yu and R.O Ritchie, Metallurgical Transactions A, 19 A, 549 (1988)

11. X He, Effect of temperature, impurities (Fe), and alloy elements (Ni, Mo, $\mathrm{Pd})$ on the crevice corrosion of alpha titanium alloys, Thesis (2003)

12. D. D Macdonald, C English, S. J Lenhart, Report to DOE/BES. SRI International, Menlo Park, CA (1985)

\section{III-4 Task: Electrochemical Emission Spectroscopy}

In this section, the examination of electrochemical noise $(\mathrm{EN})$ data comprises three main approaches: one, a computer simulation of the anodic and cathodic activity relating to corrosion on a metal surface; two, experimental modeling of the electrochemical environment inside nuclear waste storage containers and collection of EN generated; and three, Wavelet analysis (WA) of the EN data from the first two parts.

The simulation of EN is effective in replicating the EN data of both general and pitting corrosion. Using competition mechanisms for the anodic and cathodic sites on the surface, the long-term, low-frequency data generated by localized pitting corrosion is reproduced. Disabling one or more of the rules of the simulation eliminates the lowfrequency character of the data, and eliminating all of the rules effectively reproduces general corrosion noise.

WA identifies when general corrosion is the prevailing corrosion type because it has an inherently high frequency. Pitting corrosion is similarly identified by its lower 
frequency. WA also identifies when there is a mix of corrosion types by examining the contributions from both low- and high-frequency crystals to the total signal energy. Depictions of the wavelet coefficients in the time domain identify at what time a corrosion type becomes dominant or fades based on when the standard deviation of the wavelet coefficients increases or drops, respectively. It also accurately determines the average duration for many corrosion events, and it resolves the repassivation times of single events.

WA also confirms that nitrate ions are effective in inhibiting both general and localized corrosion, while nitrate ions are less effective at inhibiting localized corrosion and instead promote general corrosion. Phosphate ions promote general corrosion, and chloride ions induce pitting.

\section{$\underline{\text { Task Status: }}$}

\section{III-4.1: Computer Simulation of the Anodic and Cathodic Activity}

The goal of this portion of the research is to use the electrochemical noise from a simulation of anodic and cathodic sites on a material's surface to learn how the surface chemistry and the interaction between sites affect EN.

The primary design objective is to simulate a portion of a surface undergoing general or pitting corrosion. The simulated surface is just one small portion of a relatively infinite specimen surface, and that specimen surface is just one of two identical electrodes. The simulated surface is modeled by a two-dimensional array of sites. The sites can have one of three characters: anodic, cathodic, or neutral (passive). The simulation emulates the activity on the anodic electrode of the pair of specimen electrodes. Normally, two such identical electrodes would have exactly the same character, i.e., the same anodic and cathodic current densities on the surface, but through slightly uneven mixing, or a number of other driving forces, one electrode becomes more anodic that the other. For this simulated surface, a combination of a greater number of anodic sites or the greater magnitude of the anodic current (compared to the cathodic current) results in a higher anodic current density. 
While the direction and magnitude of current fluctuations at any time on a site have been assumed to be random (within general limits), the overall trend is deterministic in that it depends on a logical interaction between sites. According to the Coupled Environmental Fracture Model (CEFM), anodic and cathodic sites on a surface are coupled. Current flowing between the two non-neutral site types is the only way to maintain their non-neutral characters. An anodic site, whether it is a metastable or stable pit, or a crevice, must be "fed" by one or more cathodic sites. Figure III-4.1 shows the nature of the interaction between an anodic site and cathodic surface sites [2].

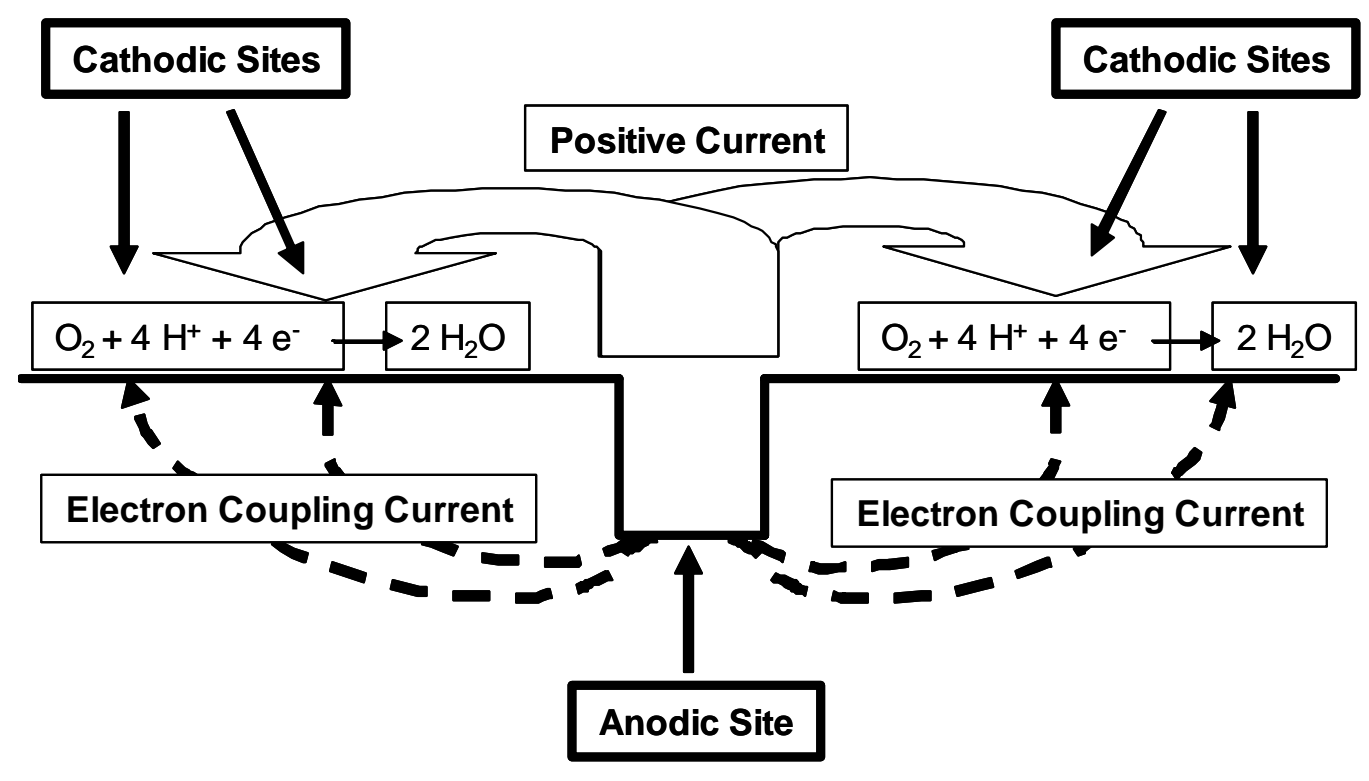

Figure III-4.1. Anodic/cathodic site interaction on a material's surface.

As long as there are enough places to consume the current from the anodic site, it will survive. There are many competitive mechanisms that impede the survival of an anodic site [1]. For the purposes of this simulation, the sites obey the following rules: the number of anodic sites is initially slightly greater than the number of cathodic sites (this creates an overall anodic character for the surface); cathodic sites have an increased probability of being located near anodic sites as opposed to being isolated or near only other cathodic sites (the probability increases with decreasing average distance from anodic sites); cathodic sites die off if there is not an anodic site nearby (within three sites 
distance); there is a small randomized chance of cathodic site death (representing factors not included in this model, like a sudden lack of resources for the cathodic reaction); anodic sites die off if there are not enough cathodic sites locally to consume the electrons produced.

Once the sites and their interactions over time have been established, the total current is calculated using the Tafel equation. Equations $\mathbf{0 . 1}$ and $\mathbf{0 . 2}$ give the total anodic and cathodic currents, respectively:

$$
\begin{aligned}
& I_{a}=A_{a} n_{a} i_{0, a} e^{E / b_{a}} \\
& I_{c}=A_{c} n_{c} i_{0, c} e^{E / b_{c}}
\end{aligned}
$$

where: $A_{a}, A_{c}$ are the anodic and cathodic areas, respectively; $n_{a}, n_{c}$ are the initial number of anodic and cathodic sites per unit area, respectively; $\mathrm{i}_{0, \mathrm{a}}, \mathrm{i}_{0, \mathrm{c}}$ are the initial anodic and cathodic current densities, respectively; $\mathrm{E}$ is the potential; $\mathrm{b}_{\mathrm{a}}, \mathrm{b}_{\mathrm{c}}$ are the anodic and cathodic Tafel slopes, respectively

The total current, $\Delta \mathrm{I}$, can be derived from Equations $\mathbf{0 . 1}$ and $\mathbf{0 . 2}$ (a form of the ButlerVolmer equation):

$$
\Delta I=A_{a} i_{0, a} e^{\left(E_{c o r r}+\Delta E\right) / b_{a}}-A_{c} i_{0, c} e^{-\left(E_{c o r r}+\Delta E\right) / b_{c}}
$$

where: $\mathrm{E}$ is equal to the corrosion potential, $\mathrm{E}_{\text {corr }}$, plus the over-potential, $\Delta \mathrm{E}$ When $\Delta \mathrm{E} / \mathrm{b}_{\mathrm{a}}$ and $\Delta \mathrm{E} / \mathrm{b}_{\mathrm{c}}$ are much, much less than one, Equation $\mathbf{0 . 3}$ becomes: 


$$
\begin{aligned}
\Delta I=A_{a} i_{0, a} e^{E_{\text {corr }} / b_{a}}\left(1+\Delta E / b_{a}\right) & \\
& -A_{c} i_{0, c} e^{-E_{\text {corr }} / b_{c}}\left(1-\Delta E / b_{c}\right)
\end{aligned}
$$

where: $\Delta \mathrm{E}$ is the overpotential for the total current, $\Delta \mathrm{I}$

To simulate the entire system, both electrodes would need to be reproduced. In that case, each surface would operate under the same rules as given above, the current generated by each surface would be generated by identical processes, and only the final magnitude and character (positive or negative) of the current would differ. The total current for the simulation would be the sum of the two individual electrode sums. In this way, simulating both surfaces is no more effective than simulating a single surface of twice the area. Since it is a linear combination of current values, and addition is commutative, for the purposes of this simulation, it does not matter how the final sum is obtained. Ultimately, only one surface was simulated.

Given values for the initial anodic and cathodic currents, the corrosion potential, and the Tafel slopes, both the total current and total voltage can be calculated in the simulation. The basic values needed for the calculation of the total current and voltage values for the simulated surface came from literature on investigation of electrochemical properties of corrosion on carbon steel [3]. The values used in the simulation are: corrosion potential, $\mathrm{E}_{\mathrm{corr}}=-0.612 \mathrm{~V}$, cathodic Tafel slope, $\mathrm{b}_{\mathrm{c}}=0.315(\mathrm{~V})$, anodic Tafel slope, $\mathrm{b}_{\mathrm{a}}=0.330 \mathrm{~V}, \mathrm{i}_{0, \text { corr }}=3.10 \mathrm{E}-6\left(\mathrm{~A} / \mathrm{cm}^{2}\right)$.

\section{III-4.1a Program Design}

The simulation program was written to work from a console application interface (similar to MS-DOS). It was written in $\mathrm{C}++$ and consists of four main source files: main.cpp which contains the driver for the program, the random noise simulation code, and was written entirely by the author; four1.cpp and realft.cpp, proprietary software files purchased under license from Cambridge University as part of their Numerical Recipes 
software package which perform Fourier transforms; and mods.cpp, which contains the code that moves the program beyond a simple random simulation of noise to code that attempts to model some of the metastable and stable pitting characteristics of localized corrosion. Additional header files necessary for mods.cpp and the Numerical Recipes source files were also used.

The file main.cpp contains the function "main()" which is the driver program. It allows the simulation and Fourier transform of the data to be run multiple times in a single session. It calls the function "standard()" which interacts with the user. The user provides the program with the number of times the program will iterate. The function "standard()" creates the individual files for output, then it creates the array that holds the simulated current data. The simulation uses a two-dimensional grid of 20 rows and 20 columns as the default size of the simulated surface. Individual locations are randomly set to anodic, cathodic, or neutral sites. The user is then prompted for the use of the modifications that simulate pitting events, or a random noise simulation. The program calls a number of functions from mods.cpp to do this. The functions are mainly responsible for managing the interaction between sites. Anodic and cathodic sites are born or die, both according to the rules given above. The function "CountCurr()" calculates the total current in the simulation every iteration and the results are saved to a text file, currents.txt. The function "GetFile()" is then called which takes a file input by the user, which calls "realft()", a Numerical Recipes function, to perform a Fourier transform of the data, and then "GetFile()" outputs the result to fourier.txt.

The program has a number of restrictions that must be considered when using it. The calculations to be made later for the Fourier transformations require that the number of data must be equal to a power of two, so this simulation limits the number of points to powers of two. The maximum number of data is restricted by the capabilities of the machine running the software, and in this case was limited to 262,144 points per signal for computation time considerations. 


\section{III-4.1b Results}

\section{Random Noise Simulation}

The random noise simulation has only a few rules governing the initial placement of sites, and current and voltage fluctuations. It creates a random arrangement of anodic and cathodic sites, and these initial positions remain unchanged throughout the simulation, but the anodic and cathodic current values for each site fluctuate randomly

with time. The result is a current record that under visual inspection appears to be a general corrosion electrochemical record. One random run of 2,048 points over 204.8 seconds (at $10 \mathrm{~Hz}$ ) is shown in Figure III-4.2.

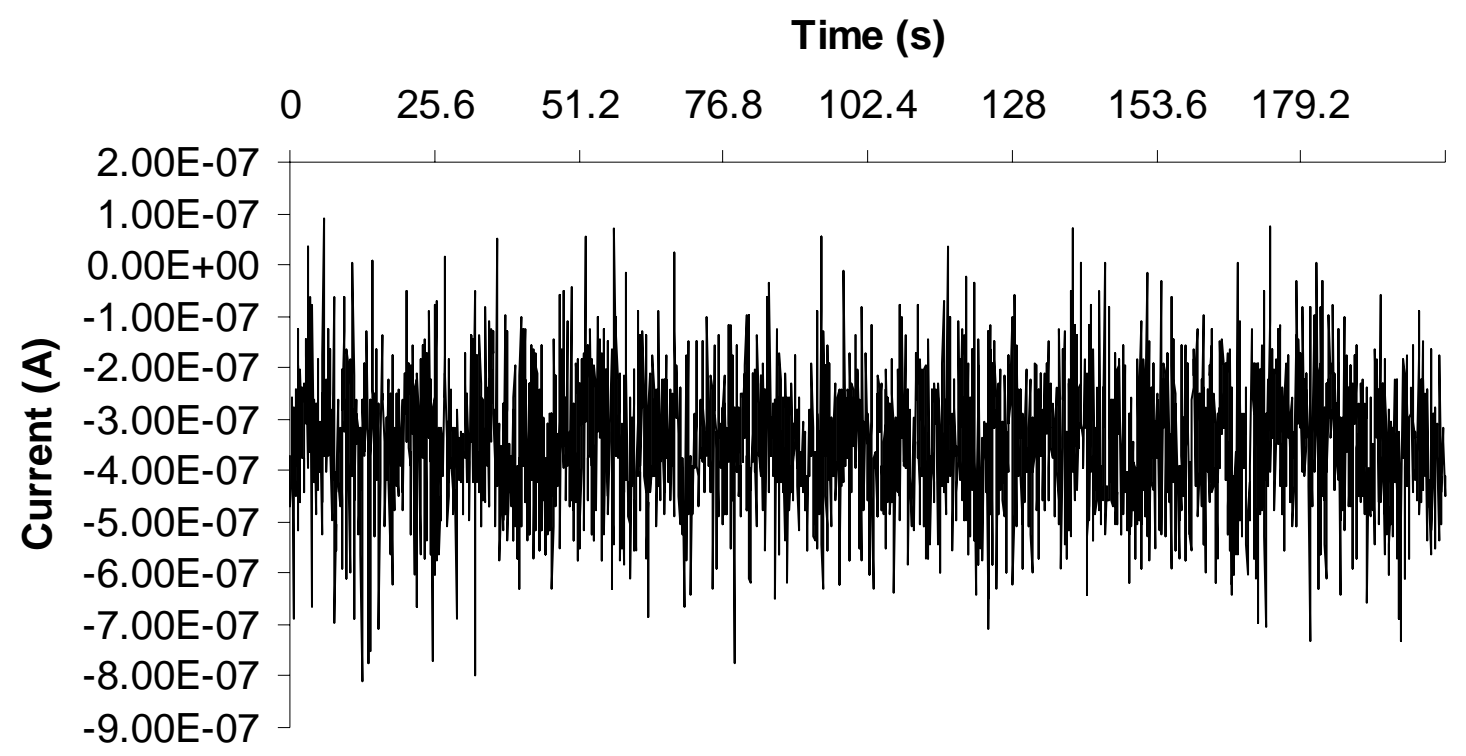

Figure III-4.2. Random EN data simulation, Run A

\section{Pitting and Rule-based General Corrosion Simulation}

A sample rule-based general corrosion run, Run B, is shown in Figure III-4.3. Visually it appears somewhat similar to the random noise simulation. A clear difference between the two is that the rule-based simulation fluctuations aren't as large in magnitude (relative to the total amplitude of the signal). Also, the rule-based simulation has peaks 
and troughs that correspond to the fluctuations in number of cathode and anodic sites. Further explanation of the work done with the simulation, and wavelet analysis of the current will be provided in Section 5.6.

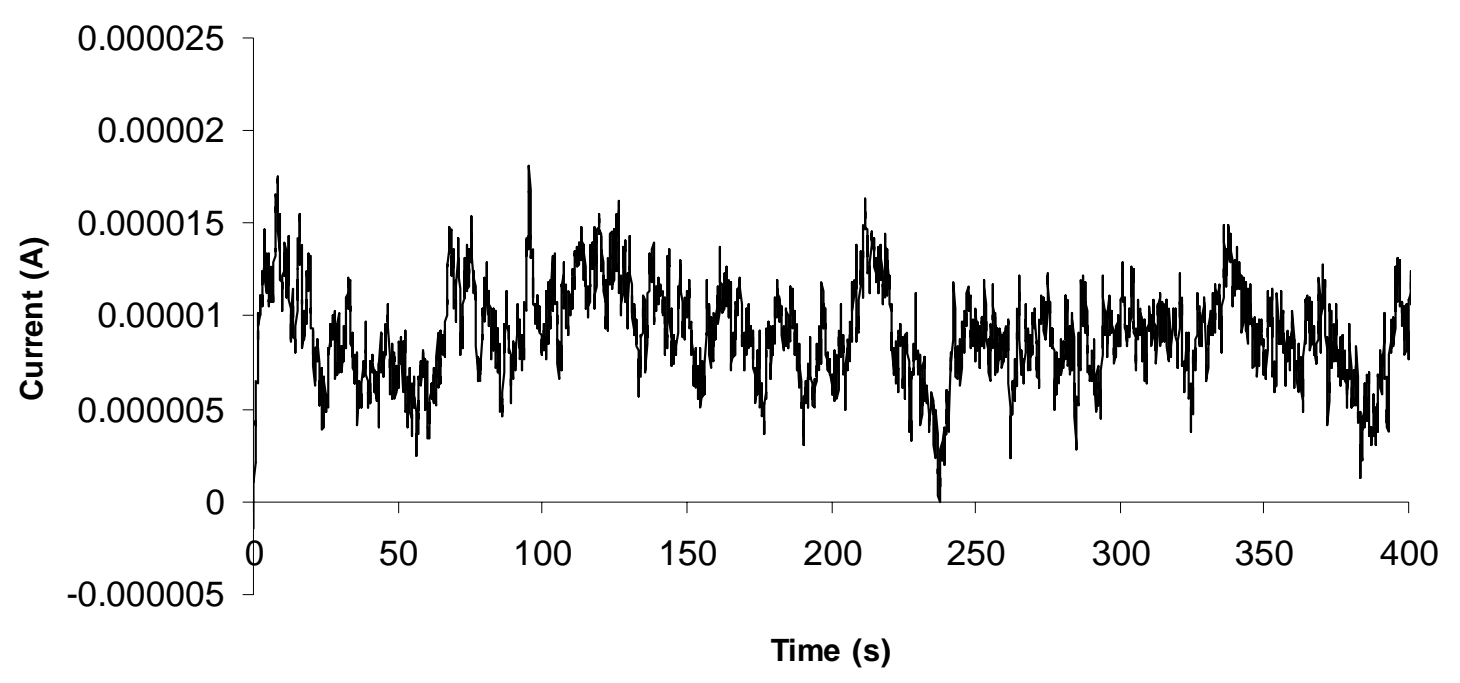

Figure III-4.3. Rule-based general corrosion simulation, Run B; individual fluctuations do not have as large a magnitude as in the general corrosion simulation, and there are peaks and troughs in the data corresponding to the birth and death of anodic and cathodic sites. 


\section{III-4.2 Experimental Modeling of the Electrochemical Environment}

The objective of the experimental work is to simulate, as much as feasible, the conditions within a high-level nuclear waste storage container. Comprehensive tests on nuclear waste are often performed on either samples of the waste (often low-level waste) or on sludges or saltcakes. These are meant to simulate many conditions, such as ionic and molecular concentrations, as well as general chemistry. The focus of this research is on the effects of corrosion of the steel containers. Therefore, conditions that affect the kinetics (the rate or severity) of the corrosion and its onset and duration are simulated. The major conditions simulated are: elevated temperature and the presence of corrosion inducing or impeding ions. Specifically, experimental runs were made at a constant $70^{\circ} \mathrm{C}$ and sodium nitrate $\left(\mathrm{NaNO}_{3}\right)$, sodium nitrite $\left(\mathrm{NaNO}_{2}\right)$, sodium chloride $(\mathrm{NaCl})$, and sodium phosphate $\left(\mathrm{Na}_{2} \mathrm{HPO}_{4}\right)$ were added to a basic solution of sodium hydroxide $(\mathrm{NaOH})$.

\section{III-4.2a Cell Design}

The electrochemical noise experiments were performed inside a double-walled glass cell. The inner wall encompasses a volume of approximately $175 \mathrm{ml}$. The outer wall wraps around the inner to provide space for heated water to be passed, in order to maintain the cell at an elevated temperature. The cell was also built with gas ports for the inlet and outlet of argon used to de-aerate the solution and to provide for some mixing. A port with a rubber septum allowed for injection of chemicals without changing the atmosphere in the cell, and another port allowed for the placement of a mercury thermometer to measure the cell temperature.

The cell was designed for a three-electrode system. Two identical carbon steel (ASTM A516) electrodes one inch in length with 1/8 inch diameters were fashioned. They were screwed onto the ends of $2 \mathrm{~mm}$ copper rods. The length of the electrodes and the copper rods were initially coated with MicroStop, leaving only the cross-sectional surface exposed to the solution. After multiple runs under pitting conditions, the coating began to suffer and delaminate at the exposed metal surface's edge. After that, the

electrodes were cleaned of the MicroStop and placed inside an epoxy mold. Leco 
Corporation polyester resin and hardener (part numbers 811-191 and 811-194, respectively), in a ratio of $30 \mathrm{ml}$ to 15 drops, were used to bind the two electrodes. As before, only the cross-sectional areas of the electrodes were exposed.

A $\mathrm{Ag} / \mathrm{AgCl}$, sat. $\mathrm{KCl}$, reference electrode and a $\mathrm{Pt}$ pseudo-reference electrode were used for collecting voltage data. The cell was sealed with a Teflon top, rubber oring, and a double c-clamp. The various ports were provided for by threaded or smoothbored holes drilled in the top and sealed with either threaded Teflon and polyethylene plugs or rubber stoppers. The entire cell was placed inside a Faraday cage to limit external electromagnetic interference.

\section{III-4.2b Chemical Mixtures}

$5 \mathrm{M}$ sodium hydroxide solution was prepared by massing 199.94 grams of sodium hydroxide using a Denver Instrument Company XE Series, Model 300 balance. It was mixed in $1000 \mathrm{ml}$ beaker with de-ionized water using a Fisher Scientific Model 11-50075 stirring hotplate. The final solution volume was $1 \mathrm{~L}$.

The other four solutions were mixed in the same manner to provide their respective bulk molarities. Table III-4.1 shows the bulk solution concentrations, and the volumes of each injection (depending on the order of injection of the added 4 chemicals), and the target concentration in the cell. The target concentrations were calculated for the ions directly after mixing. The concentrations would decrease over time with the additional volume of later injections. High bulk concentrations were used so that there would be minimum volume change with each injection. The concentrations were limited in the cases of sodium nitrite and sodium nitrate by their maximum solubilities at room temperature. 
Table III-4.1: Combination runs, A-D; shows order of addition, volume used, target concentration after addition, and the concentration of the bulk solution from which the volumes were taken.

\begin{tabular}{|c|c|c|c|c|c|}
\hline Run & Chemical & Order & $\begin{array}{c}\text { Volume } \\
(\mathrm{ml})\end{array}$ & $\begin{array}{l}\text { Target } \\
\text { Conc. }\end{array}$ & $\begin{array}{l}\text { Bulk } \\
\text { conc. }\end{array}$ \\
\hline \multirow[t]{5}{*}{ A } & $\mathrm{NaNO}_{2}$ & 3 & 7.0 & 0.50 & 8 \\
\hline & $\mathrm{NaNO}_{3}$ & 4 & 8.6 & 0.50 & 7 \\
\hline & $\mathrm{NaCl}$ & 2 & 5.3 & 0.10 & 2 \\
\hline & $\mathrm{NaOH}$ & 1 & 100.0 & 5.0 & 5 \\
\hline & $\mathrm{Na}_{2} \mathrm{HPO}_{4}$ & - & - & - & - \\
\hline \multirow[t]{5}{*}{ B } & $\mathrm{NaNO}_{2}$ & 4 & 7.6 & 0.50 & 8 \\
\hline & $\mathrm{NaNO}_{3}$ & 3 & 8.1 & 0.50 & 7 \\
\hline & $\mathrm{NaCl}$ & 2 & 5.3 & 0.10 & 2 \\
\hline & $\mathrm{NaOH}$ & 1 & 100.0 & 5.0 & 5 \\
\hline & $\mathrm{Na}_{2} \mathrm{HPO}_{4}$ & - & - & - & - \\
\hline \multirow[t]{5}{*}{ c } & $\mathrm{NaNO}_{2}$ & 5 & 7.6 & 0.50 & 8 \\
\hline & $\mathrm{NaNO}_{3}$ & 3 & 8.1 & 0.50 & 7 \\
\hline & $\mathrm{NaCl}$ & 2 & 5.3 & 0.10 & 2 \\
\hline & $\mathrm{NaOH}$ & 1 & 100.0 & 5.0 & 5 \\
\hline & $\mathrm{Na}_{2} \mathrm{HPO}_{4}$ & 4 & 0.6 & 0.01 & 2 \\
\hline \multirow[t]{5}{*}{ D } & $\mathrm{NaNO}_{2}$ & 4 & 7.6 & 0.50 & 8 \\
\hline & $\mathrm{NaNO}_{3}$ & 2 & 7.7 & 0.50 & 7 \\
\hline & $\mathrm{NaCl}$ & 3 & 5.7 & 0.10 & 2 \\
\hline & $\mathrm{NaOH}$ & 1 & 100.0 & 5.0 & 5 \\
\hline & $\mathrm{Na}_{2} \mathrm{HPO}_{4}$ & - & - & - & - \\
\hline
\end{tabular}

The target concentrations of the individual chemicals were determined from literature on the chemistry of sampled waste storage containers [5-8]. The solution chemistry of sodium nitrate and sodium nitrite varied over quite a range in the literature, so an intermediate value of $0.5 \mathrm{M}$ was chosen for each. Phosphate concentration was universally low, and $0.01 \mathrm{M}$ was chosen. Chloride ion concentration was chosen to be 0.1 
M, based on the waste literature and literature describing the role of chloride ions in inducing pitting on carbon steel [9]

\section{III-4.2c Experimental Procedure}

Prior to all data runs, the components of the cell, including tubes, electrodes, thermometer, and plugs, and all the glassware involved were washed with soap and water, then rinsed. All were then rinsed again with distilled water and flushed using deionized water for periods up to half an hour. These steps insured that the presence of ions (corrosion inducing or otherwise) was reduced as much as possible.

The working and counter electrodes were polished prior to every run using 320 grit polishing paper. They were polished in the same direction each time (parallel to the alignment of the electrodes in the epoxy) for two minutes or until the electrode surfaces appeared unblemished to the eye.

The starting solution for each run consisted of $100 \mathrm{ml}$ of $5 \mathrm{M} \mathrm{NaOH}$. This volume of liquid was placed inside the cell, and the cell was sealed. A solid rubber stopper took the place of the electrode plug for the preliminary steps. The solution was de-aerated prior to all runs with $99.9 \%$ pure argon gas for at least 30 minutes. A Fisher Scientific water heater/cooler/re-circulator (model 9101) was used to pump heated water through the outer cell. The water temperature was set to $70^{\circ} \mathrm{C}$, and it took approximately 15 minutes to reach that value starting from room temperature. Upon reaching the set temperature, a mercury thermometer was used to verify the temperature within the cell. The temperature of $70^{\circ} \mathrm{C}$ was steady over course of the runs.

Upon reaching the set temperature, the rubber stopper with the electrodes replaced the solid stopper and data collection was begun. Data runs for general corrosion lasted from 5 to 15 hours. The electrochemical data were collected using a Gamry PC3 card using ECA400 software and an EN Analyzer v. 1.20. Two leads from the Gamry card were connected to the working electrode, and two were connected to the counter electrode. For runs where voltage data were collected, an additional lead was connected to each of the reference and the working electrodes. Figure III-4.4 shows the arrangement of leads for the standard zero-resistance ammeter (ZRA) mode. 


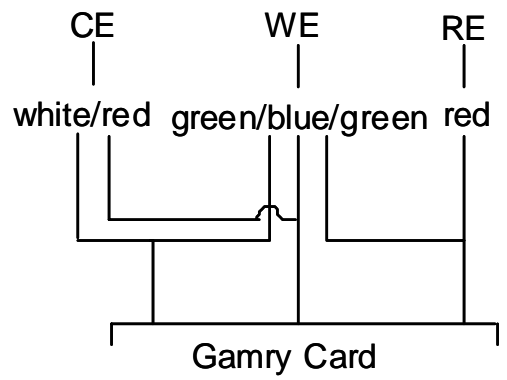

Figure III-4.4. Depiction of the Gamry lead setup for ZRA mode; CE is the counter electrode, WE is the working electrode, and RE is the reference electrode.

At the beginning of each run, the current flowing between the two specimen electrodes was checked using a Keithley 485 autoranging picoammeter to determine the magnitude. The hardware of the Gamry card was then set the highest precision setting that would accurately measure the current flow (the available options were orders of magnitude: 3 $\mathrm{nA}, 30 \mathrm{nA}, 0.3 \mu \mathrm{A}, 3 \mu \mathrm{A}$, etc). Furthermore, a picoammeter reading of $0.3 \mu \mathrm{A}$ would require the hardware setting to be $3 \mu \mathrm{A}$, because if the hardware was set to only $0.3 \mu \mathrm{A}$, any fluctuations above $0.329 \mu \mathrm{A}$ would not be read by the card.

For data runs involving simple general and pitting corrosion conditions, the $2 \mathrm{M} \mathrm{NaCl}$ solution was used. After the electrodes were placed in the standard solution, the cell temperature reached the designated working temperature, and the electrochemical noise from the general corrosion stabilized (56 minutes were allowed for stabilization and recording of general corrosion noise), $5.3 \mathrm{ml}$ of $2 \mathrm{M} \mathrm{NaCl}$ solution was injected into the cell, making the final concentration of chloride ions $0.1 \mathrm{M}$. Pitting corrosion data were recorded for at least 2 hours after injection.

For data runs involving a combination of corrosion conditions varying over time, all five solutions were used in differing amounts, as shown in Table III-4.1. The $5 \mathrm{M} \mathrm{NaOH}$ solution always formed the foundation solution. The remaining four solutions were added in different orders. The interaction of the solutions and their effects on each other were the goal of these runs. In each case, data were collected for 56 minutes before the next chemical was added. The results of all runs are covered in the next chapter. 


\section{III-4.2b Optical Microscopy}

To confirm the presence of pits, the metal surface was examined using optical microscopy after chloride injection and observation of pitting transients in the current data. Figures III-4.5 and figure III-4.6 are images taken with an optical microscope [Olympus BX60M with Polaroid DMC Ie v. 1.25 image software]. The first image shows a collection of pits on the surface, and the second image shows a higher magnification of pair of deeper pits. Pit A contains highly reflective corrosion product.

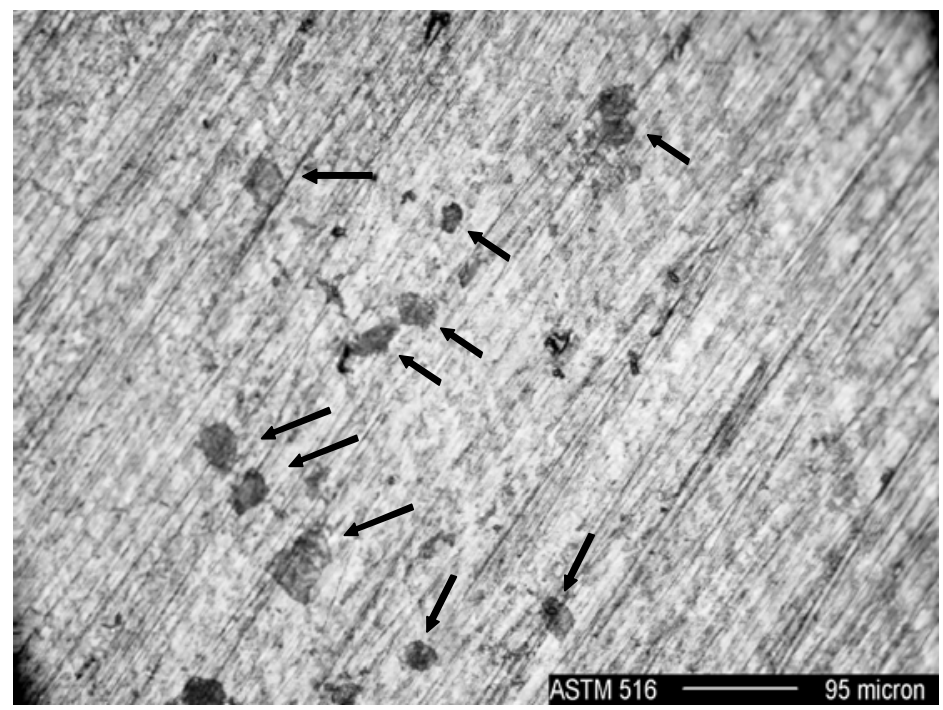

Figure III-4.5. Several pits (arrowed) on the surface of the working electrode after chloride injection. 


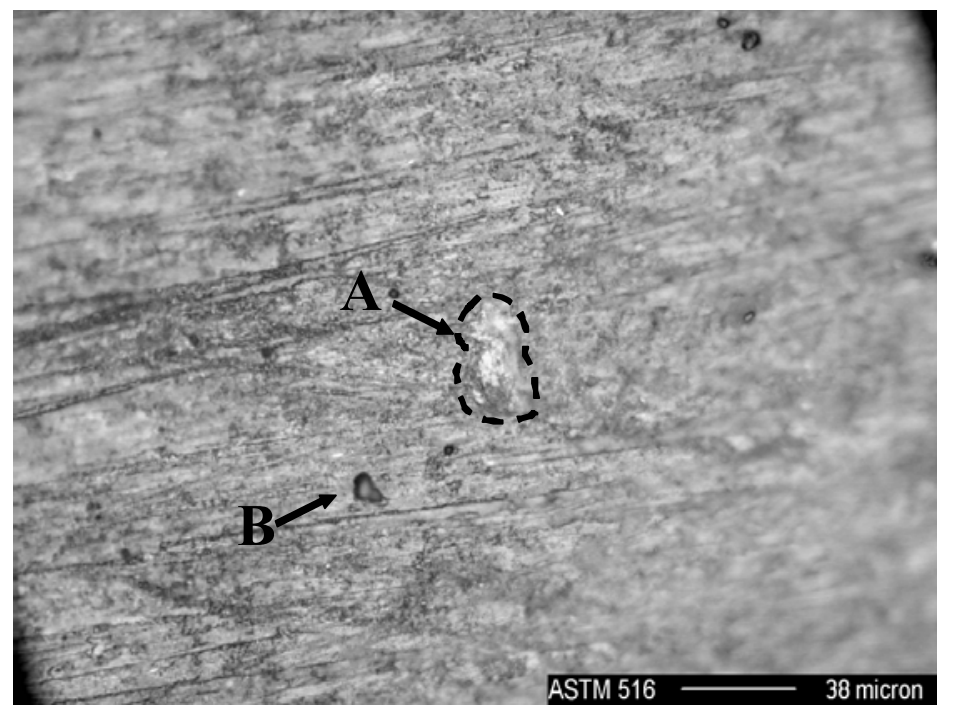

Figure III-4.6. Close-up of two pits on the surface; A is a deep pit with highly reflective corrosion product, and B is a deep pit with little product. 


\section{III-4.3 Wavelet Analysis (WA) of the EN Data}

The wavelet functions used in this study are the Daubechies wavelets. The particular set is known as DAUB4, and has only four coefficients [10]. This makes these wavelets highly localized in time, good for EN studies where short time duration events are the norm. The operation of the four coefficients can be depicted in a matrix format, shown in Figure III-4.7.

$$
\left[\begin{array}{cccccccccccc}
c_{0} & c_{1} & c_{2} & c_{3} & & & & & & & \\
c_{3} & -c_{2} & c_{1} & -c_{0} & & & & & & & \\
& & c_{0} & c_{1} & c_{2} & c_{3} & & & & & \\
& & c_{3} & -c_{2} & c_{1} & -c_{0} & & & & & \\
& & & & & & \ddots & & & & \\
& & & & & & & c_{0} & c_{1} & c_{2} & c_{3} \\
& & & & & & & c_{3} & -c_{2} & c_{1} & -c_{0} \\
c_{2} & c_{3} & & & & & & & & c_{0} & c_{1} \\
c_{1} & -c_{0} & & & & & & & & c_{3} & -c_{2}
\end{array}\right]
$$

Figure III-4.7. Matrix of Daubechies Coefficients

The matrix is square and its total dimensions are dependent on the number of points in the experimental data set. The matrix is orthogonal and thus is constrained by Equations 0.1 and 0.2 [10]:

$$
\begin{aligned}
& c_{0}^{2}+c_{1}^{2}+c_{2}^{2}+c_{3}^{2}=1 \\
& c_{2} c_{0}+c_{3} c_{1}=0
\end{aligned}
$$

These two equations are not sufficient to solve for the values of the four coefficients, so an approximation condition must be applied. The matrix in Figure 4 can be thought of as having two filters: $H=\left(c_{0}, c_{1}, c_{2}, c_{3}\right)$ and $G=\left(c_{3},-c_{2}, c_{1},-c_{0}\right)$; $H$ being the smoothing filter and $G$ being the wavelet (detail) filter. The values for the 
coefficients in $\mathrm{G}$ must be chosen so that $\mathrm{G}$ responds to the smoothed data as minimally as possible, and this is done by making the moment of $\mathrm{G}$ equal to zero. The nth moment of $\mathrm{G}$ is the average value of the elements of $\mathrm{G}$ raised to the $\mathrm{n}^{\text {th }}$ power, given in Equation $\mathbf{0 . 3}$ :

$$
m^{n}=\frac{\sum_{i=0}(-1)^{i+1} c_{i}}{N} \approx 0
$$

where: $\mathrm{N}=4$, the number of coefficients in $\mathrm{G}$ When $\rho$ moments equal zero, then it is said to satisfy an approximation condition of order $\rho$. The approximation condition of order $\rho=2$ provides the following Equations $\mathbf{0 . 4}$ and $0.5[10]$ :

$$
\begin{aligned}
& c_{3}-c_{2}+c_{1}-c_{0}=0 \\
& 0 c_{3}-1 c_{2}+2 c_{1}-3 c_{0}=0
\end{aligned}
$$

With these four equations, the values of the coefficients can be found. The solutions are irrational numbers. In this research, the precision of these numbers was limited to 16 decimal points for use in a computer program.

To perform the wavelet transform, the matrix is multiplied by the vector containing the experimental data, and this produces an array of the same length, $\mathrm{X}$, as the initial data set, containing half as many "smooth" coefficients, $\mathrm{X} / 2$, and half as many “detail" coefficients, X/2.

This first set of detail coefficients gives information on the features with the highest frequencies. Successive multiplications with the smooth coefficients of each previous product produce additional sets of detail coefficients. Each set is at half the resolution (the minimum resolvable frequency is twice as large) and contains half the number of coefficients. Once there are only two smooth coefficients remaining, the "mother function coefficients," [10] the process is complete. The final detail coefficients 
provide information as to how much each wavelet function contributed to the signal at each resolution and at each (time domain) moment. To reconstruct the original signal, the process is simply reversed.

The interval range of each crystal is given by Equation 0.6 [5]:

$$
\left(I_{1}, I_{2}\right)=\left(2^{j} \Delta t, 2^{j-1} \Delta t\right)
$$

where: $\mathrm{I}_{1}=1 / \mathrm{f}_{1}, \mathrm{I}_{2}=1 / \mathrm{f}_{2}$

The results of the analysis of the wavelet coefficients are depicted in two ways. First is an Energy Density Plot (EDP). The total energy contained within the original signal can be calculated with Equation 0.7:

$$
E=\sum_{i=1}^{N} x_{i}^{2}
$$

where: $\mathrm{N}$ is the total number of coefficients, $\mathrm{x}_{\mathrm{i}}$ are the individual wavelet coefficient values

The fraction of the total energy in a given crystal can then be calculated using Equations 0.8 and 0.9 :

$$
\begin{gathered}
E_{j}^{d}=\frac{1}{N} \sum_{i=1}^{N / 2 j} d_{j, i}^{2} \\
E_{j}^{s}=\frac{1}{N} \sum_{i=1}^{N / 2} s_{j, i}^{2}
\end{gathered}
$$

where: $\mathrm{d}$ or $\mathrm{s}$ indicates a detail crystal or a smooth crystal, respectively; $\mathrm{j}$ is the number assigned to each crystal (eg., D1, D2, etc.)

Comparing the percentage of energy for each crystal against other crystals in that data set and against counterpart crystals from other data sets will illustrate the power of WA to differentiate between corrosion types and to qualify severity. 
The second way the results of WA will be depicted will be a graph of all the coefficients for crystals of a signal in the time domain against the original signal. It is this depiction that will illustrate the power of WA to retain temporal information, and to identify the onset or termination of corrosion processes.

\section{III-4.3a Aliasing}

Aliasing is a problem that occurs regardless of the use of Fourier Transforms or Wavelet analysis because it in inherent to the signal acquisition. The problem arises when infinite, continuous data are sampled in a discrete, bandwidth-limited manner. The Sampling Theorem provides the upper and lower limits for sampling frequencies that will be properly sampled in a given recording. Equations $\mathbf{0 . 1 0}$ and $\mathbf{0 . 1 1}$ provide these limits:

$$
\begin{gathered}
f_{\max }=\frac{1}{2 \Delta t} \\
f_{\text {min }}=\frac{2}{T}
\end{gathered}
$$

where: $\Delta \mathrm{t}$ is the sampling interval, $\mathrm{T}$ is the total time of sampling

Equation $\mathbf{0 . 1 0}$ is also known as the Nyquist critical frequency. If the signal contains only frequencies less than this frequency, then the signal can be completely determined by sampling at that rate. If the signal being sampled consists of some frequencies outside of these limits, those frequencies will be erroneously "aliased" into the frequency range. The aliased data cannot be extracted from the analysis while retaining the true data because they are inseparable once recorded.

There are several practical methods of preventing aliasing. One is to control the upper limit of frequencies in the incoming signal using filters. An ideal, low-pass filter would eliminate any frequencies above the critical frequency, and thus aliasing would not be a problem. Practical filters are not ideal because they are not perfectly abrupt at the specified frequency, but they are a good working solution.

A second way to deal with aliasing is often used with Fourier transforms. That method is

to treat the original signal as a continuous, repeating signal ${ }^{5}$ then discard the aliased 
frequencies. This repetition is useful for analyses where the number of points after analysis needs to be the same as the number in the original sampling. The method of doubling is used with Fourier transforms because it is affected by DC drift, and Fourier transforms are limited to data with a constant mean.

There is also a degree of wraparound caused by the WA algorithm. This is similar to aliasing in that it can affect the resultant data, but it is an artifact of the algorithm and not a result of sampling. It can be eliminated by changes to the algorithm. In the case of the WA used in this study, shifting the columns of Figure III-4.7 N/2 positions to the left to symmetrize the wraparound (seen here in the coefficients in the first two columns of the last two rows) largely reduces aliasing. To eliminate the wraparound completely, the coefficients in the first and last $\mathrm{N}$ rows can be altered to create a band-diagonal matrix [10].

In this case, the aliasing was not eliminated using low-pass filters, so the highest 10 percent of frequencies (those frequencies affected by aliasing [11]) were discarded from analysis. The lowest frequencies (positive frequencies approaching zero) were not affected by aliasing and were not eliminated.

\section{III-4.3b Program Design and Implementation}

The design of the WA software is relatively simple. The program was written to work from a console application interface similar to MS-DOS. The program was written in $\mathrm{C}++$ and consists of three main source files: main.cpp which contains the driver for the program, the bulk of the code, and was entirely written by the author, wt1.cpp and daub4.cpp are proprietary software files [purchased under license from Cambridge University as part of the Numerical Recipes software package] which perform the actual wavelet transform. Additional header files required by the Numerical Recipes source files were also used.

The file main.cpp contains the function "main()" which is the driver program. It allows the WA to be run multiple times in a single session. It calls "WaveCalc()" which has the job of the primarily interaction with the user. The user provides the program with the subdirectory name, the name of the text file (eg. currents.txt) that contains the current 
data as taken over time, the number of data in the file, and the frequency of data acquisition. "WaveCalc()" then calls the Numerical Recipes function "wt1()" which does the wavelet transformation. "WaveCalc()" then calls "XtalCalc()" to determine from the wavelet coefficients the fraction of total energy per crystal (as discussed above). "XtalCalc()" calculates the fraction of total energy for each crystal. It also calculates the approximate place in the time domain for the coefficients of each crystal, relative to the original signal. Then it outputs the time, frequency ranges, wavelet coefficients, and original current data to a text file based on the name of the file the user specified. Once this is done, it returns the fractional crystal energy totals to "WaveCalc()", which outputs them to a separate file: crystal.txt. All output files are placed in the original directory specified by the user.

The program has a number of preconditions that the file containing the current data must fit. First, the file must be a text file containing only the current data from the beginning. The wavelet transform calculations require that the number of data points must be equal to a power of two, but after that number of entries in the file, the remaining entries may be any data the user wishes, because the program will ignore it. The maximum number of points is restricted by the capabilities of the machine running the software, and, in this case, was limited to 262,144 points per signal.

\section{III-4.3c Wavelet Analysis of EN in Sodium Hydroxide Solution}

Wavelet analysis of the electrochemical data collected from the specimens in sodium hydroxide solution Run A is reproduced in Figure III-4.8. This figure depicts crystals D3-D14 of the Energy Density Plot (EDP) for data collected over 3.64 hours comprising 131,072 points. 97.8 percent of the energy in Run A is tied up in the smooth data crystal- "S" in Table . Crystals 14 though 16 each contain only tenths of a percent of the total energy, indicating that there is some activity in the frequency ranges of 0.0000763 to $0.00122 \mathrm{~Hz}$ (the long-term signals) which is indicative of the DC drift in the raw current data, shown in Figure III-4.9. The rest of the crystal energy contributions are even lower. This is an indication that there is very little activity occurring on this sample, and that the steel is passive under $5 \mathrm{M}$ sodium hydroxide conditions. 


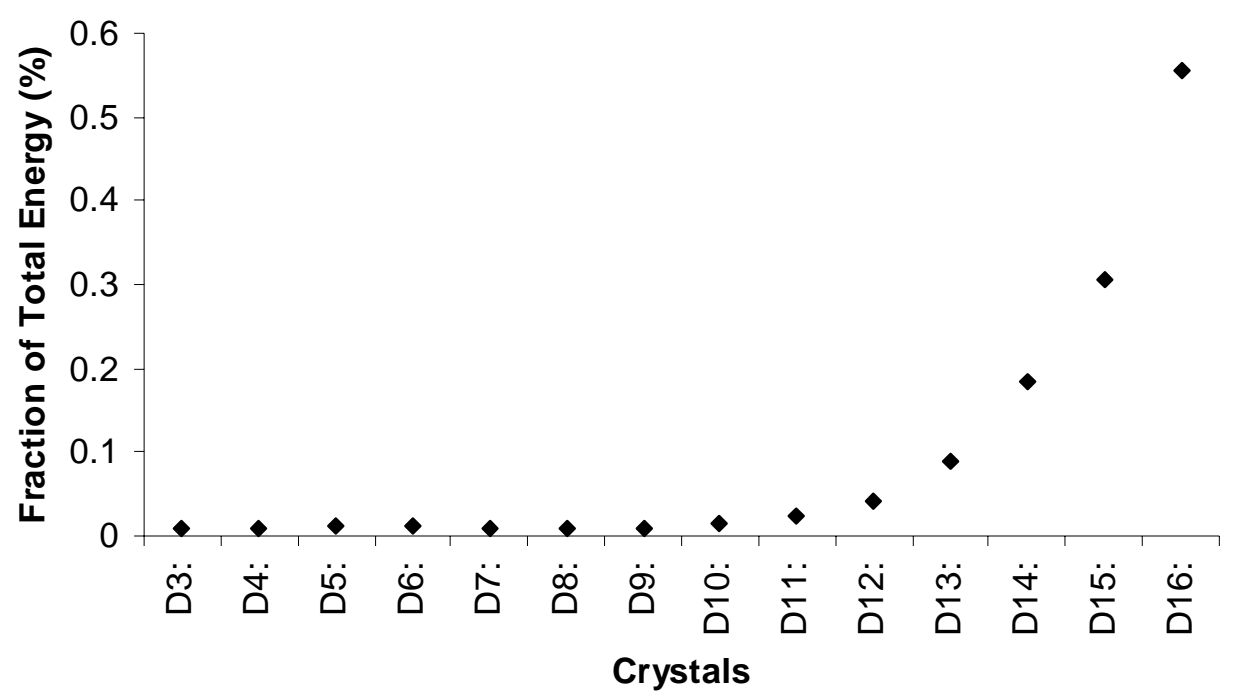

Figure III-4.8. Sodium hydroxide solution, Run A: wavelet analysis identifies no major corrosion contributions, indicating that the steel specimen is passive under $5 \mathrm{M}$ sodium hydroxide conditions.

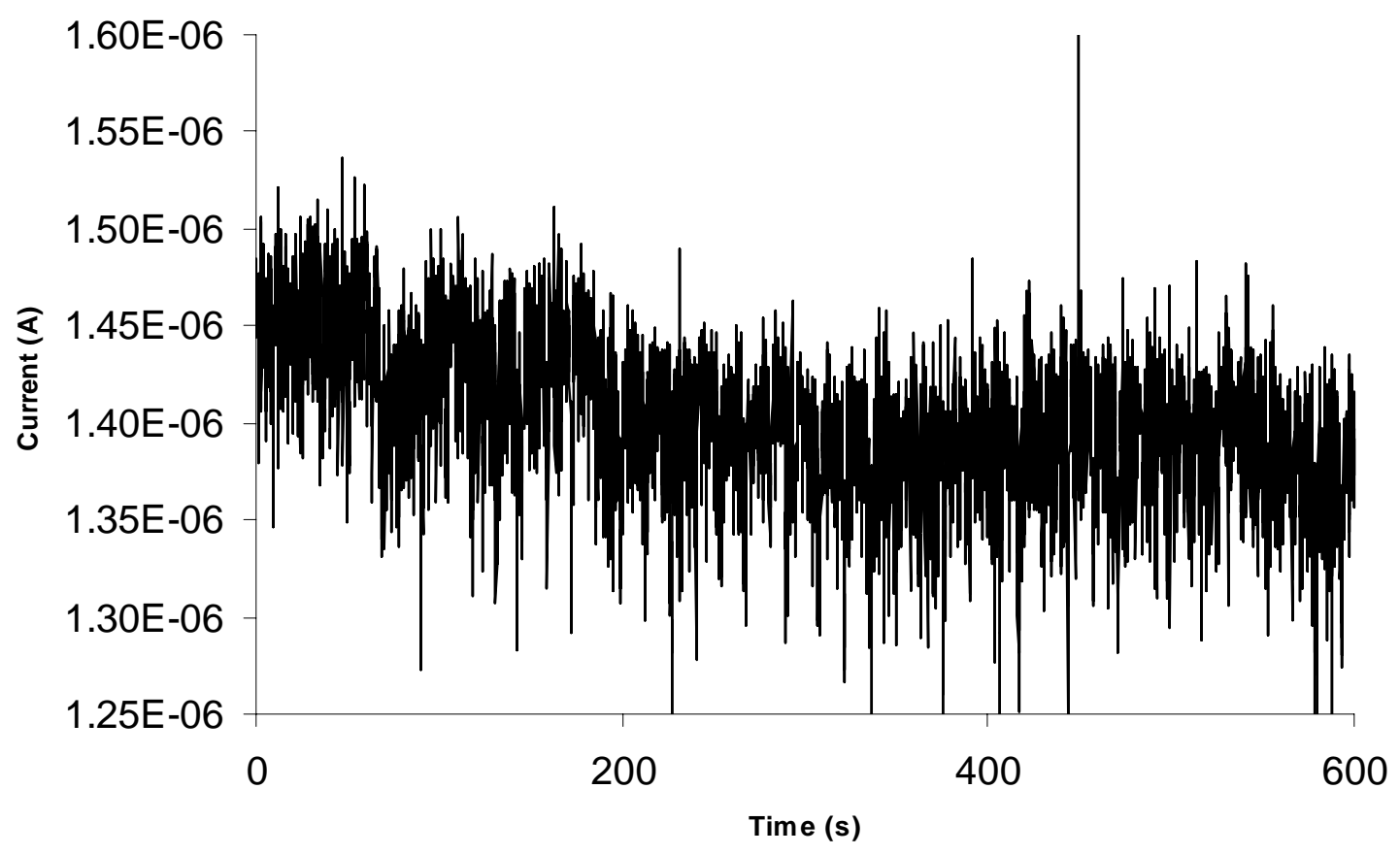


Figure III-4.9. Sodium hydroxide solution, Run A; illustrates DC drift in the current record over 600 seconds contributing to the low-frequency energy totals.

Figure III-4.10 shows data from a second run in sodium hydroxide solution. The data were again taken over 3.64 hours comprising 131,072 points. Another large percentage of the energy resides in the smooth data (95.2 percent), but this time the highfrequency crystals, D3-D7, contain significant fractions of the total energy. This highfrequency activity was identified as periodic external electrical interference, which will be discussed in detail in pitting corrosion Run $\mathrm{D}$, in Section 5.4.

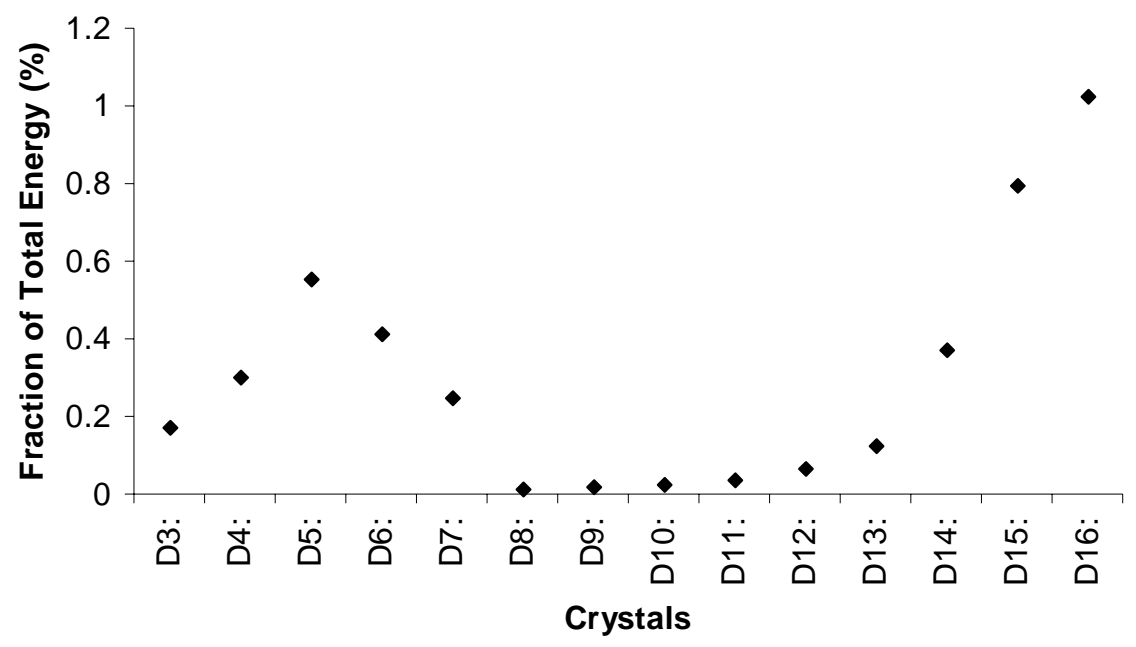

Figure III-4.10. Sodium hydroxide solution, Run B; the increased energy of crystals D3-D7 is the result of an extraneous electrical signal discussed below in pitting corrosion Run D.

The EDP percentages for all sodium hydroxide and pitting corrosion runs are given in Table III-4.2 and Table III-4.3. 
Table III-4.2: Fractional energy contribution (\%) for all crystals; sodium hydroxide conditions, Runs A and $\mathrm{B}$, and pitting corrosion Runs A-C.

\begin{tabular}{|c|c|c|c|c|c|c|}
\hline & \multicolumn{2}{|c|}{ Sodium Hydroxide Runs } & \multicolumn{2}{|c|}{ Pitting Corrosion Runs } & \multirow[b]{2}{*}{ B } & \multirow[b]{2}{*}{ C } \\
\hline & $A$ & B & $A$ & & & \\
\hline Crystals & & & series 1 & series 2 & & \\
\hline D3: & 8.74E-03 & 1.70E-01 & 1.19E-02 & 2.76E-02 & 1.77E-02 & $9.71 \mathrm{E}-03$ \\
\hline D4: & 7.49E-03 & 3.01E-01 & $9.50 \mathrm{E}-03$ & 1.73E-02 & $1.88 \mathrm{E}-02$ & $9.46 \mathrm{E}-03$ \\
\hline D5: & 1.05E-02 & $5.52 \mathrm{E}-01$ & 1.02E-02 & 1.15E-02 & 1.35E-02 & 1.79E-02 \\
\hline D6: & 1.09E-02 & 4.11E-01 & 1.46E-02 & 9.69E-03 & 1.42E-02 & 2.81E-02 \\
\hline D7: & 1.00E-02 & 2.45E-01 & 2.47E-02 & 1.55E-02 & 1.28E-02 & 2.41E-02 \\
\hline D8: & 7.78E-03 & 1.38E-02 & 5.71E-02 & 2.33E-02 & 2.39E-02 & 5.19E-02 \\
\hline D9: & $9.27 \mathrm{E}-03$ & 1.83E-02 & 1.29E-01 & 4.78E-02 & 2.68E-02 & 4.98E-02 \\
\hline D10: & 1.43E-02 & 2.53E-02 & 2.33E-01 & 7.69E-02 & 3.23E-02 & 6.72E-02 \\
\hline D11: & 2.48E-02 & 3.72E-02 & 3.92E-01 & 1.30E-01 & 1.10E-01 & 3.32E-02 \\
\hline D12: & 4.11E-02 & 6.36E-02 & 8.91E-01 & 2.89E-01 & 1.42E-01 & 9.66E-02 \\
\hline D13: & 8.99E-02 & 1.26E-01 & 8.21E-01 & 3.25E-01 & 2.71E-01 & 3.48E-01 \\
\hline D14: & 1.84E-01 & 3.70E-01 & 4.27E-01 & 1.07E-01 & 5.29E-01 & $1.42 E+00$ \\
\hline D15: & 3.07E-01 & 7.92E-01 & $1.11 \mathrm{E}+00$ & 7.83E-01 & $1.68 \mathrm{E}+00$ & $2.46 \mathrm{E}+00$ \\
\hline D16: & 5.55E-01 & $1.02 \mathrm{E}+00$ & $8.89 \mathrm{E}+00$ & $1.38 \mathrm{E}+00$ & - & - \\
\hline D17: & 8.48E-01 & - & - & - & - & - \\
\hline S: & $9.78 \mathrm{E}+01$ & $9.52 \mathrm{E}+01$ & $8.70 \mathrm{E}+01$ & $9.67 \mathrm{E}+01$ & $9.71 \mathrm{E}+01$ & $9.54 \mathrm{E}+01$ \\
\hline
\end{tabular}

Table III-4.3: Fractional energy contribution (\%) for all crystals; pitting corrosion conditions, Runs D-F.

\begin{tabular}{|c|c|c|c|c|c|c|}
\hline & \multicolumn{2}{|c|}{ Pitting Corrosion Runs } & & & & \\
\hline & $\mathrm{D}$ & & & $E$ & & $\mathrm{~F}$ \\
\hline Crystals & series 1 & series 2 & series 3 & series 1 & series 2 & \\
\hline D3: & 1.16E-02 & $9.36 \mathrm{E}+00$ & 1.05E-01 & 2.59E-02 & 3.03E-02 & 2.05E-02 \\
\hline D4: & $1.84 \mathrm{E}-02$ & $5.41 \mathrm{E}+00$ & 1.08E-01 & $5.20 \mathrm{E}-02$ & 4.97E-02 & 3.86E-02 \\
\hline D5: & 3.48E-02 & $2.88 \mathrm{E}+00$ & $1.48 \mathrm{E}-01$ & $9.04 \mathrm{E}-02$ & 8.84E-02 & 3.99E-02 \\
\hline D6: & 8.18E-02 & $1.15 \mathrm{E}+00$ & $2.48 \mathrm{E}-01$ & $1.32 \mathrm{E}-01$ & $1.54 \mathrm{E}-01$ & 2.95E-02 \\
\hline D7: & 1.87E-01 & 7.62E-01 & 3.10E-01 & 4.71E-01 & 3.34E-01 & 3.58E-02 \\
\hline D8: & 4.14E-01 & 6.51E-01 & 8.21E-01 & $1.12 \mathrm{E}+00$ & 9.06E-01 & 3.02E-02 \\
\hline D9: & 6.98E-01 & 7.84E-01 & $1.45 \mathrm{E}+00$ & $1.68 \mathrm{E}+00$ & $1.87 \mathrm{E}+00$ & 1.65E-01 \\
\hline D10: & $8.25 E-01$ & 9.73E-01 & $2.62 \mathrm{E}+00$ & $1.85 \mathrm{E}+00$ & $2.87 \mathrm{E}+00$ & 8.61E-02 \\
\hline D11: & $9.63 E-01$ & $1.31 \mathrm{E}+00$ & $2.96 \mathrm{E}+00$ & $3.50 \mathrm{E}+00$ & $3.77 \mathrm{E}+00$ & - \\
\hline D12: & 6.31E-01 & $1.92 \mathrm{E}+00$ & $3.91 \mathrm{E}+00$ & $3.32 \mathrm{E}+00$ & $4.69 \mathrm{E}+00$ & - \\
\hline D13: & 9.19E-01 & 7.03E-01 & 6.94E-01 & $4.63 \mathrm{E}+00$ & $4.19 \mathrm{E}+00$ & - \\
\hline D14: & $1.16 \mathrm{E}+00$ & $1.14 \mathrm{E}+00$ & 5.74E-01 & $1.16 \mathrm{E}+01$ & $2.57 \mathrm{E}+00$ & - \\
\hline D15: & $4.06 \mathrm{E}+00$ & $1.29 \mathrm{E}+00$ & - & $1.39 \mathrm{E}+01$ & $9.23 E-01$ & - \\
\hline D16: & $3.99 \mathrm{E}+00$ & $2.00 \mathrm{E}+01$ & - & $4.26 \mathrm{E}+01$ & $1.60 \mathrm{E}+00$ & - \\
\hline D17: & - & - & - & - & $2.53 \mathrm{E}+00$ & - \\
\hline S: & $8.60 \mathrm{E}+01$ & $1.35 \mathrm{E}+01$ & $8.58 \mathrm{E}+01$ & $1.49 \mathrm{E}+01$ & $7.34 \mathrm{E}+01$ & $9.95 \mathrm{E}+01$ \\
\hline
\end{tabular}




\section{III-4.3d Wavelet Analysis of $\mathrm{Cl}^{-}$Induced Pitting Corrosion Data}

Figure III-4.11 through Figure III-4.18 depict the EDPs for experimental runs under pitting conditions. These runs were done on a variety of time scales to show WA's effectiveness on multiple scales. Figure III-4.11 shows the EDP from two series of Run A, produced from data collected during two consecutive 3.64 hour periods. There is one key aspect of all of the pitting corrosion graphs that differs from that of the pure sodium hydroxide run graphs; certain low-frequency crystals each have higher percentages of the total energy than their counterparts did in Figures III-4.8 and III-4.10. For example, in pitting corrosion Run A, crystals D6-D16 all have increased contributions compared to the sodium hydroxide runs. Which crystals differ and by how much depends on the individual corrosion run. In Run A, the increase in crystals D9-D13 is an indication of the occurrence of pitting on the specimen. Pitting events and their repassivation have longer time intervals than do the flickering events of the anodic and cathodic sites of general corrosion, and thus their energy contributions are in lower-frequency crystals, but their time intervals are shorter than that of the DC drift so they do not contribute to the lowestfrequency crystals. Figure III-4.11 also shows a decrease in the contributions in crystals D9-D13 between series 1 and series 2. This is an identification of the decreasing occurrence of pitting events over time. Zhang, et al. [12] found similar results for pitting corrosion on pure aluminum: the contributions from the low-frequency crystals dropped off with time. 


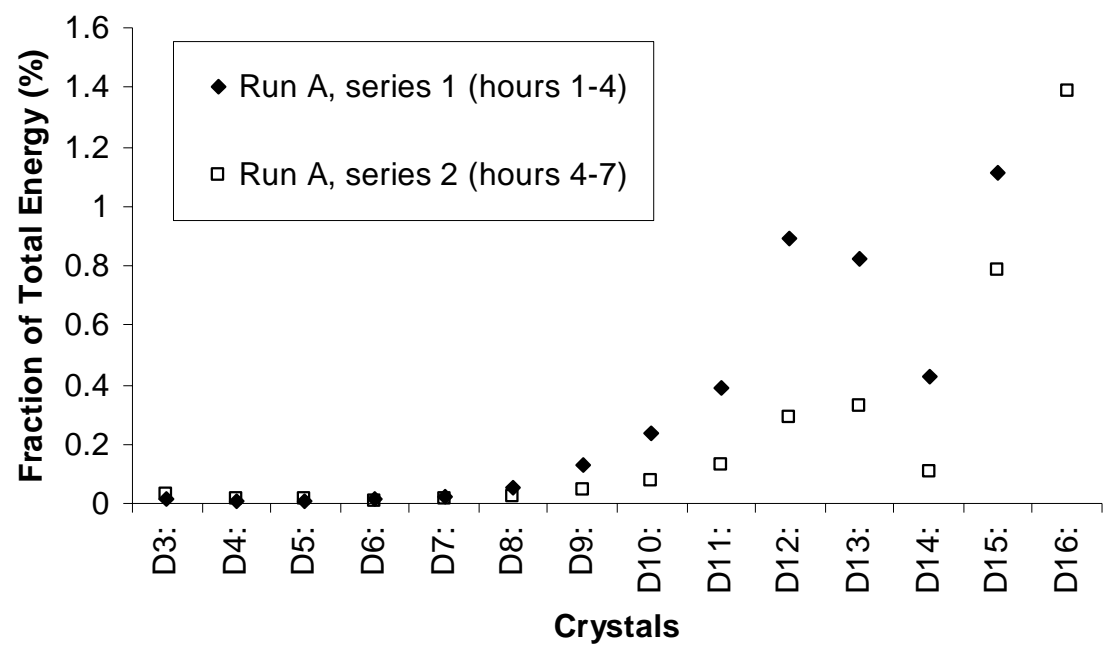

Figure III-4.11. Pitting corrosion, Run A; shows no significant high-frequency energy contributions that would be indicative of the presence of general corrosion, but shows larger energy contributions from lowfrequency crystals (D19-D13) than seen under pure sodium hydroxide conditions. This is the result of pitting corrosion. The decrease in the same crystals between series 1 (hours 1-4) and series 2 (hours 4-7) show how wavelet analysis correctly identifies and depicts the decreasing intensity of pitting corrosion with time.

Figure III-4.12 shows two more pitting runs, B and C, both over 1.82 hours comprising 65,536 points. Both show energy contribution increases in the low-frequency crystals D8-D10, and no increase in the percentages of high-frequency crystals. Again, this is the expected trend corresponding to the pitting corrosion. Examining a portion of the current record for these two runs (shown in Figure III-4.6 to Figure III4.13), it can be seen that pitting events are not as predominant in Run B as compared to Run C, which accounts for the lower fractions for D8-D10. 


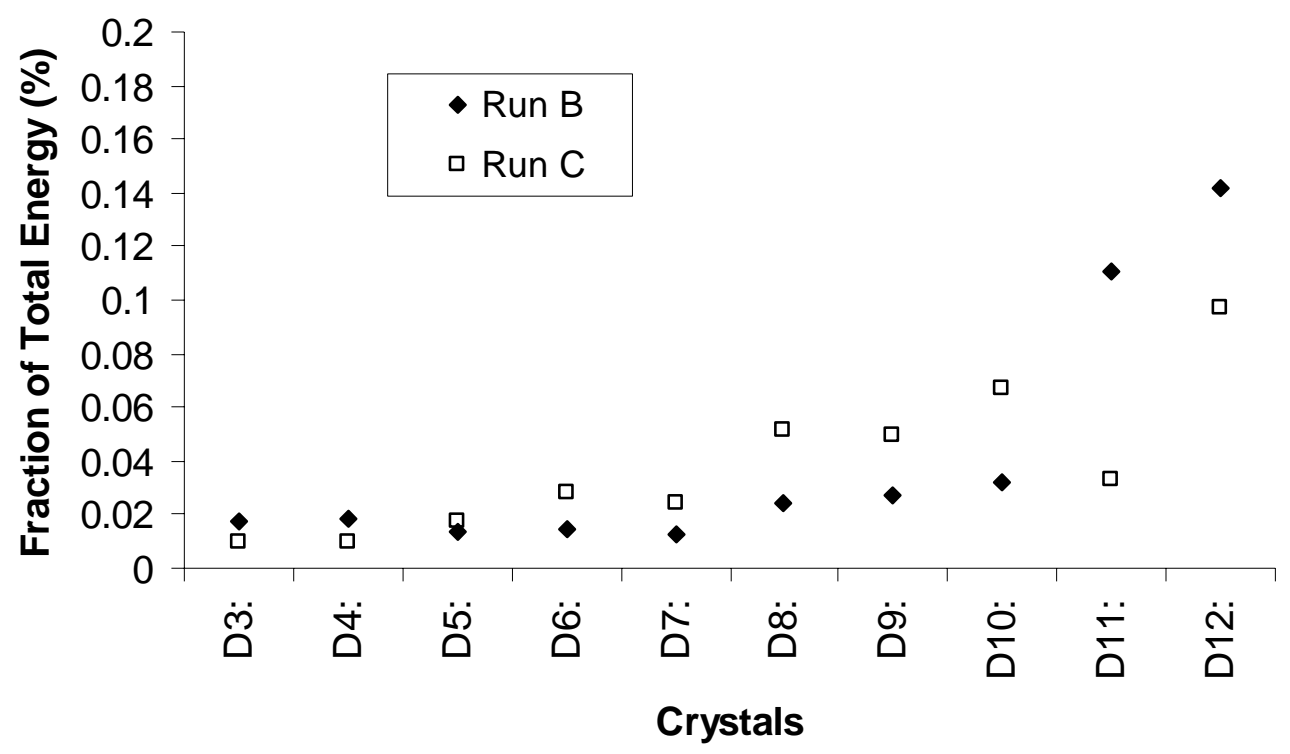

Figure III-4.12. Pitting corrosion conditions, Runs B and C; two additional pitting runs showing no increase in high-frequency energy contributions, but showing the low-frequency increase (crystals D8-D10) corresponding to pitting corrosion.

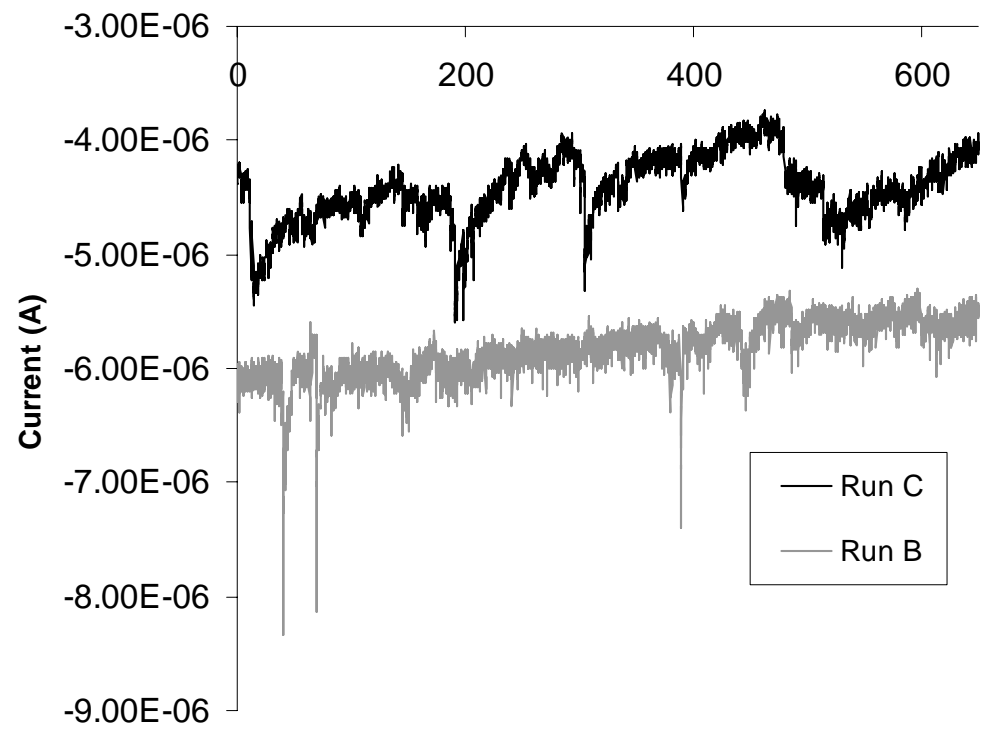

Time (s)

Figure III-4.13. Pitting corrosion conditions, Runs B and C; Portions of the current record showing the 
pitting corrosion in Run C is more intense than for Run B, which agrees with the EDP for these runs.

Figure III-4.14 was produced from data collected over 3.64 hours comprising 131,072 points. The lower-frequency crystals, D7-D11, have increased energy totals compared to the sodium hydroxide runs, and the fractional totals are on the same order of magnitude of all the other pitting corrosion runs. The large values for crystals D15 and D16 are the result of DC drift seen in the current record after the injection of chloride ions, shown in Figure III-4.6 through Figure III-4.15.

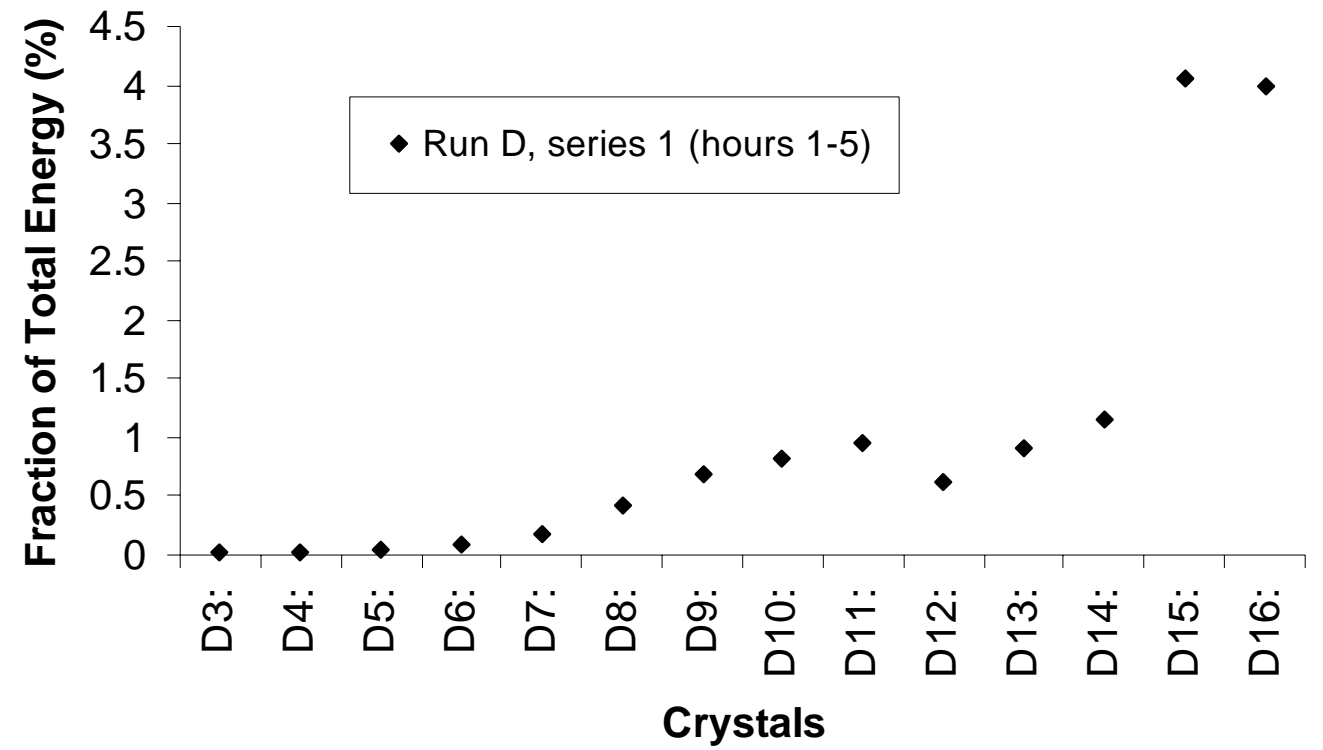

Figure III-4.14. Pitting corrosion, Run D; the low-frequency crystals starting with D7 have energy increases indicative of pitting corrosion. 


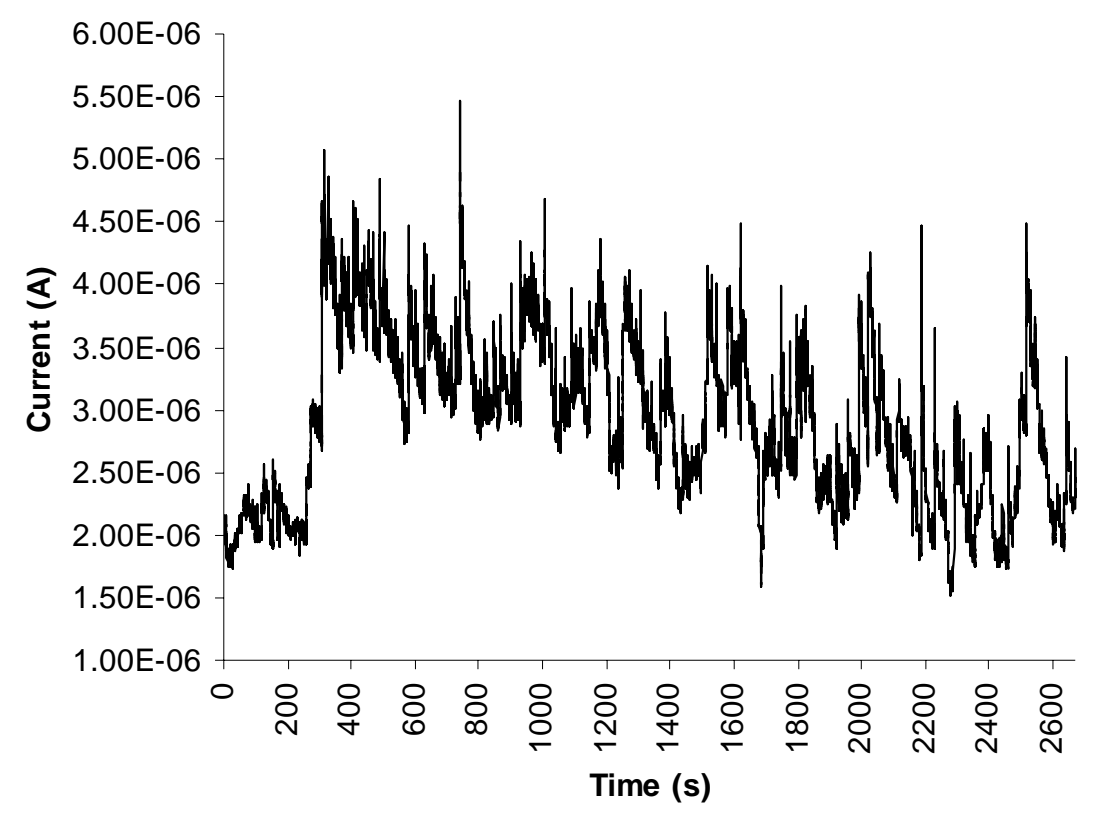

Figure III-4.15. Pitting corrosion conditions, Run D; Shows the DC drift in the current record after the injection of chloride ions at approximately $258 \mathrm{~s}$ which contributed the energy totals to crystals D15 and D16 of the EDP for this run.

The next graph for pitting corrosion is Figure III-4.16. The data shown in this graph are from a second and third series of pitting corrosion Run D. Much like Run B under sodium hydroxide conditions, the first series has an increase in the contributions of the high frequency crystals (in this case D3 through D7). This doesn't follow the established pattern for pitting corrosion analysis, though it may indicate that both general and pitting corrosion were present at the same time. Examination of the raw current data for that run showed the cause of this deviation. Despite the Faraday cage employed, an extraneous electrical signal was recorded. Figure III-4.17 depicts a sample of the data collected containing the extraneous signal. It is symmetrical and this sample has a total time interval of only 1.7 seconds, over which it fluctuates 7 times. This gives it an average frequency of $4.11 \mathrm{~Hz}$, which falls in the range of the D2 crystal (which has been discarded from all analyses due to aliasing). The frequency of the signal is not uniform during each event and differs from instance to instance, and thus it also contributes to the other high-frequency energy totals. 
Eliminating these signals from the collected data and then performing the wavelet analysis on the purified data [sampling a section of the data without the external noise would achieve the same effect] produces series 3 in Figure III-4.16. As expected, that series shows no high-frequency tail, and there is still the expected increase in the pitting crystals (D8-D12).

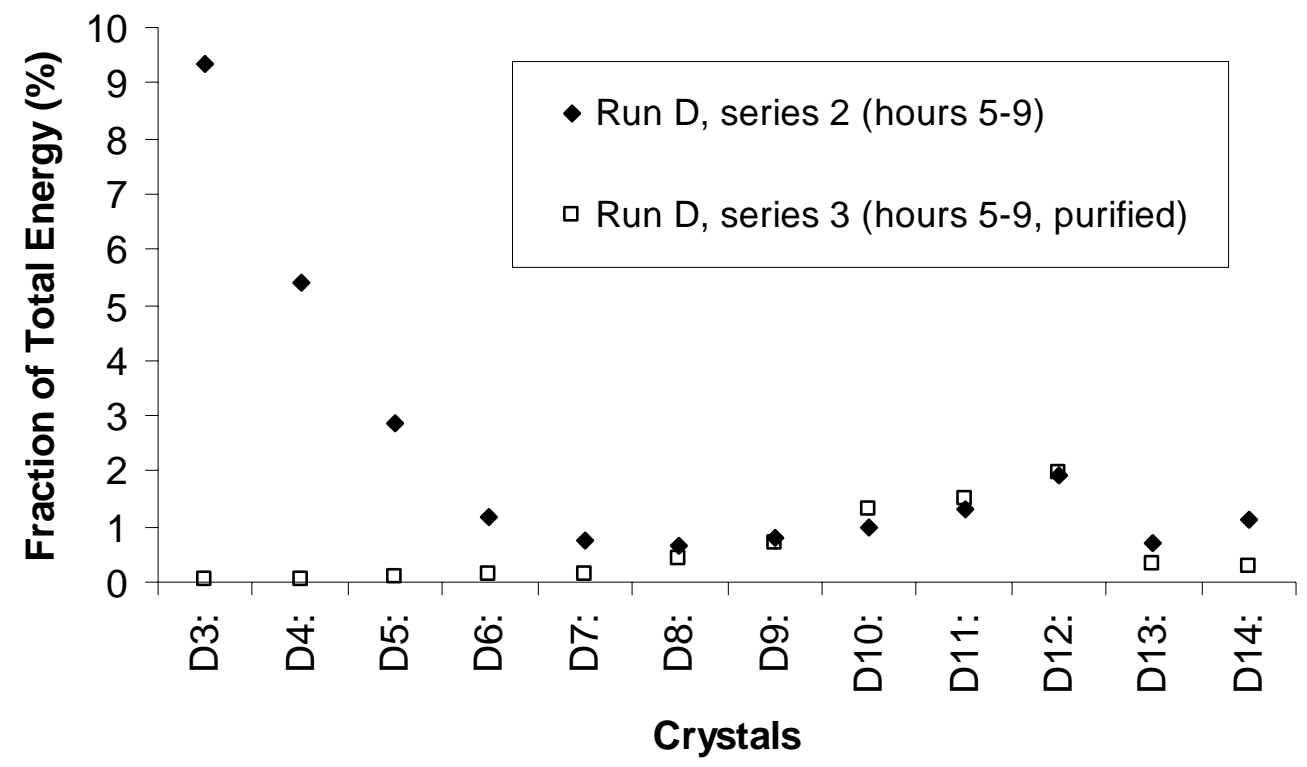

Figure III-4.16. Pitting corrosion, Run D; series 2 contains a high-frequency energy increase that might be indicative of the predominance of general corrosion, but in this case these results are due to an extraneous electrical signal depicted in Figure Error! Reference source not found.. Series 3 shows analysis of the purified EN signal showing no high-frequency contribution. 


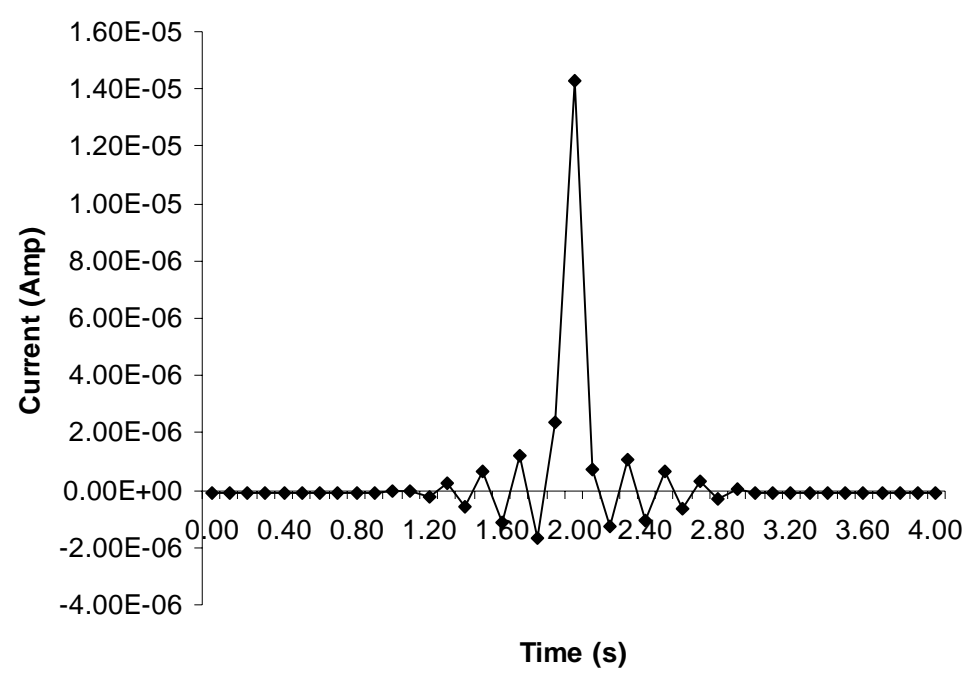

Figure III-4.17. Electrical interference in Run D; the current data for a typical extraneous event is clearly not the result of cell reactions, such as pitting, as it is highly symmetric and regular. Similar interference was found in Run B under sodium hydroxide conditions contributing to the increased high-frequency energy contributions seen in that run's EDP.

The EDP for Run E, shown in Figure III-4.18, depicts an increase in the energy contributions from crystals D8-D13. The crystal fractional energy totals for series 1 continue to increase with decreasing frequency which is a result of the large DC drift in that run. By the beginning of the seventh hour, the drift had ended, and series 2 shows only a small contribution from the lowest-frequency crystals D14-D16. 


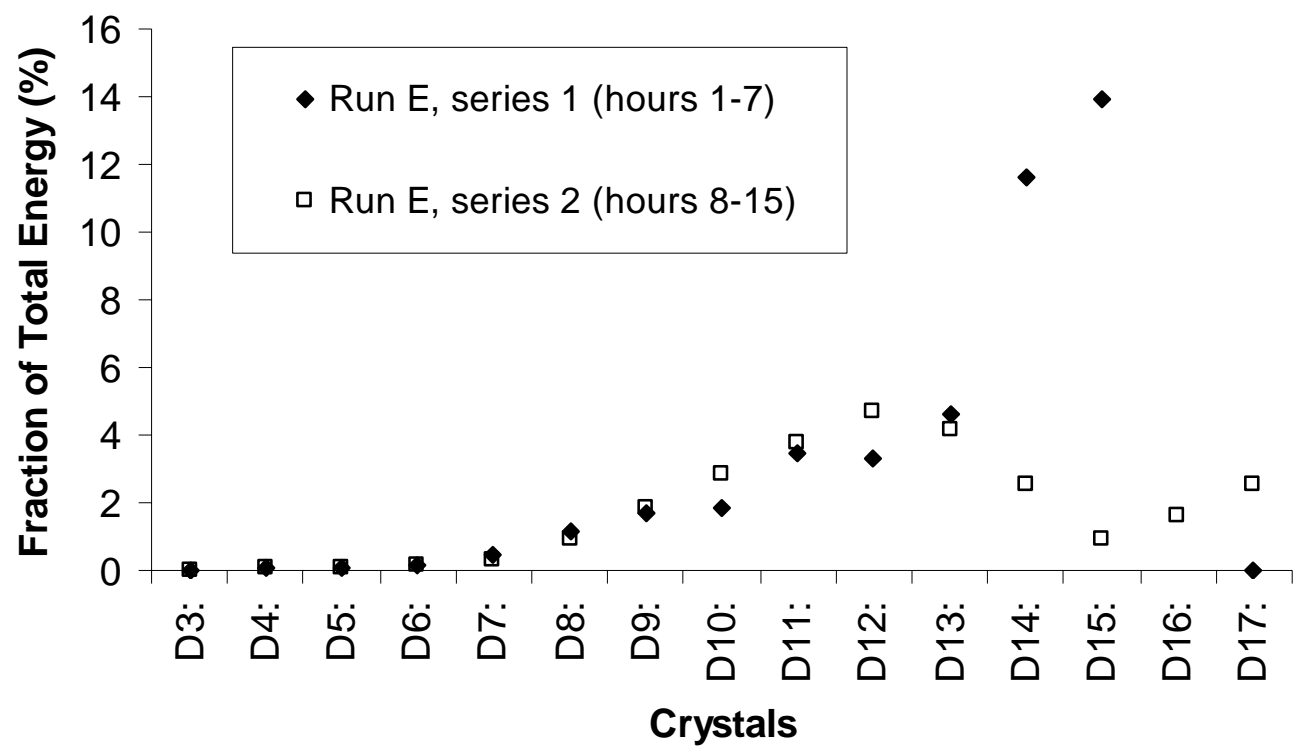

Figure III-4.18. Pitting corrosion, Run E; shows the low-frequency energy contribution increase indicative of pitting corrosion (crystals D8-D13). The presence of DC drift is identified in the first series by the large contributions from the lowest-frequency crystals, D14-D16.

Figure III-4.19 plots the detail coefficients for pitting corrosion Run F, from a data set of 2,048 points (204.8 seconds), against the original signal. Examining Figure III-4.19, it is possible to determine which frequency ranges contain events that contributed to the overall signal. An example is the large jump in the original data signal at 96.8 seconds. Crystals D3, D4, and D5 all show large coefficients at this same time. This is an example of how wavelet analysis retains the time domain information from the original signal throughout the analysis. Liu, et al [13] saw this same capability when examining the effect of various inhibitors on the pitting corrosion on aluminum. 


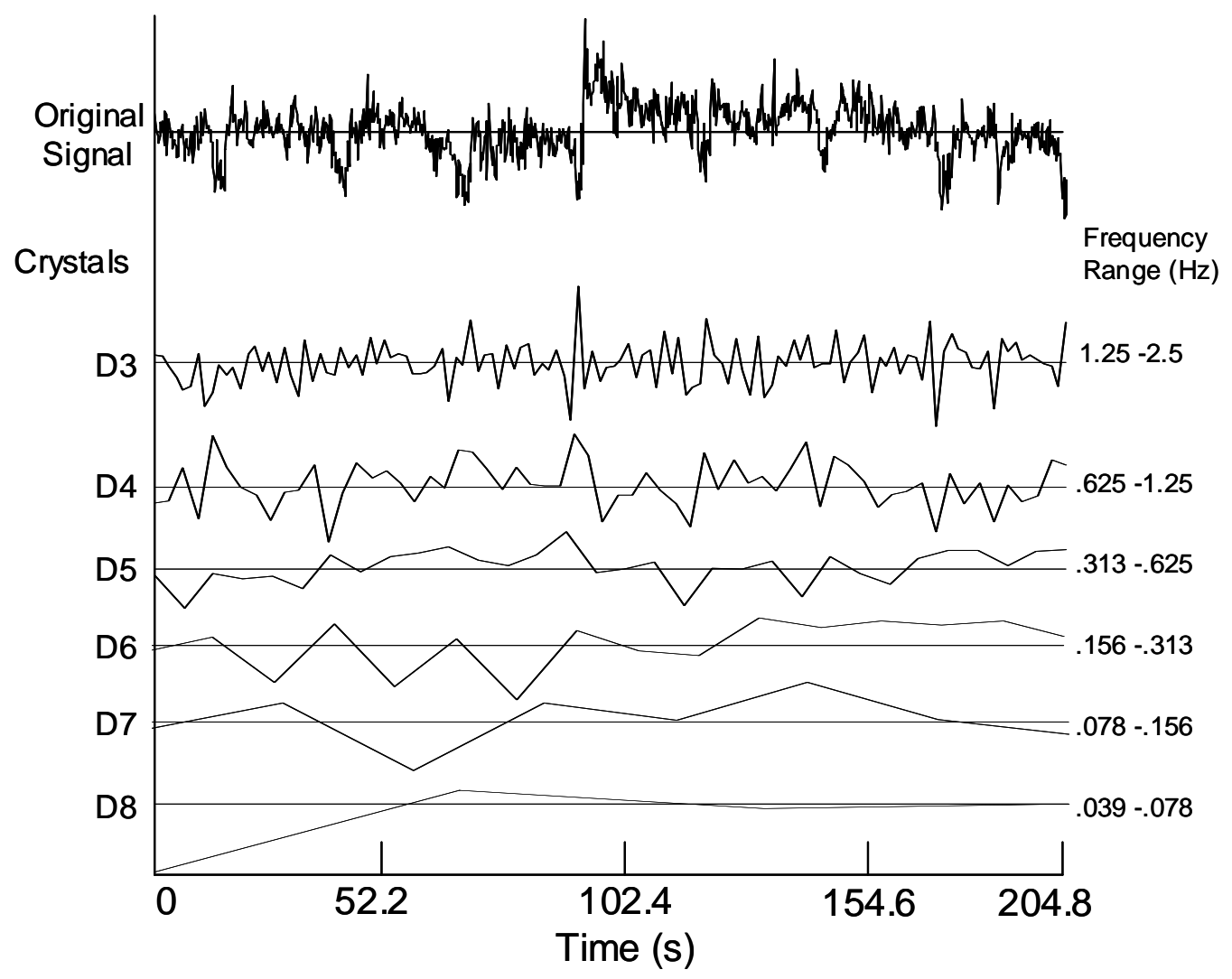

Figure III-4.19. Wavelet crystals and original signal for pitting corrosion, Run F; the ability of WA to depict the contributions of different frequency ranges to the overall signal can be seen in the time domain. The large EN event at 96.8 seconds has major contributions from frequencies 1.25-0.625, 0.625-1.25, and $0.313-0.625 \mathrm{~Hz}$, as seen in crystals D3, D4, and D5, respectively.

\section{WA of a Single Transient Event}

Figure III-4.20 shows a single large current transient with smaller overlapping transients from pitting corrosion Run D. Figure III-21 shows the first 100 seconds of the data from Figure III-4.20. Two spans of time are indicated on the figure, indicating two possible partial repassivation times for the large transient. These two times were chosen because they terminate before smaller transients occurred (at $54.7 \mathrm{~s}$ and $99.6 \mathrm{~s}$, respectively), affecting the current data. Given these times, the frequency of the repassivation would be $0.0199 \mathrm{~Hz}$ or $0.0112 \mathrm{~Hz}$, which correspond to wavelet crystals D9 and D10, respectively. The WA of the current from Figure III-4.20 is shown in Figure III4.22 , and the major peak is in crystals D9 and D10. This shows the ability of WA to 
properly analyze the repassivation of individual pitting events, and it confirms the correspondence between the low-frequency crystals (around D8 to D13) and pitting corrosion events.

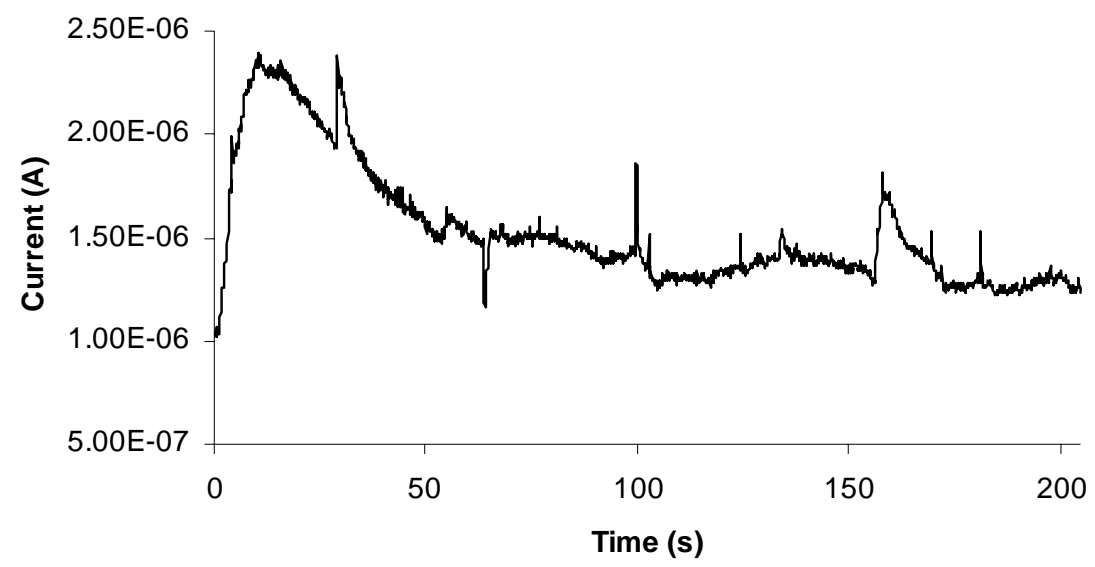

Figure III-4.20: Single large transient with smaller overlapping transients from pitting corrosion, Run D.

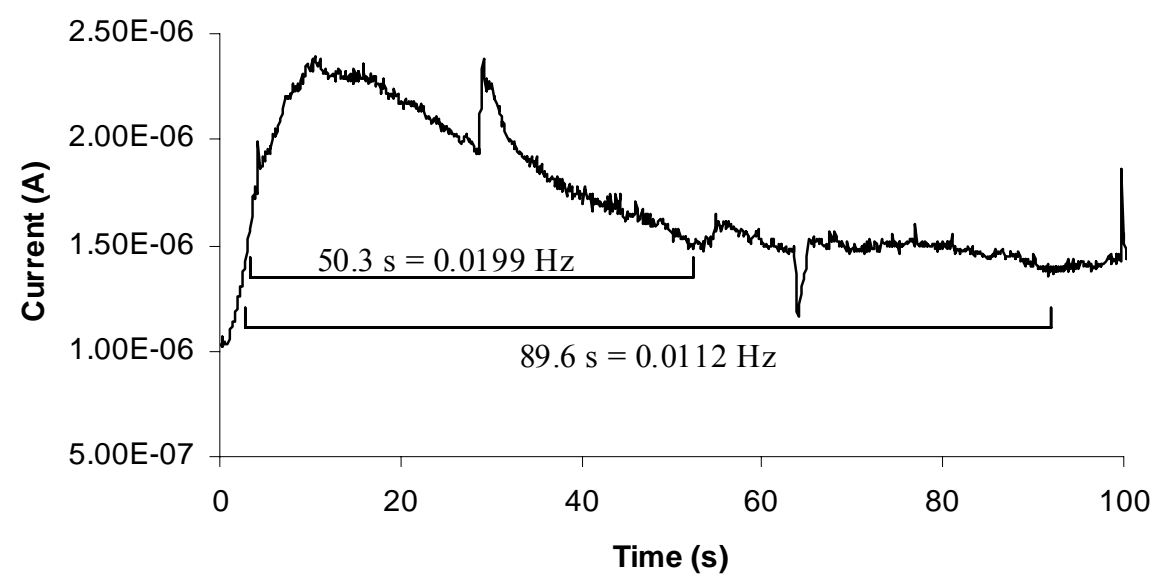

Figure III-4.21. Closer inspection of the data in Figure III-4.20; two possible repassivation times for the large transient are indicated. 


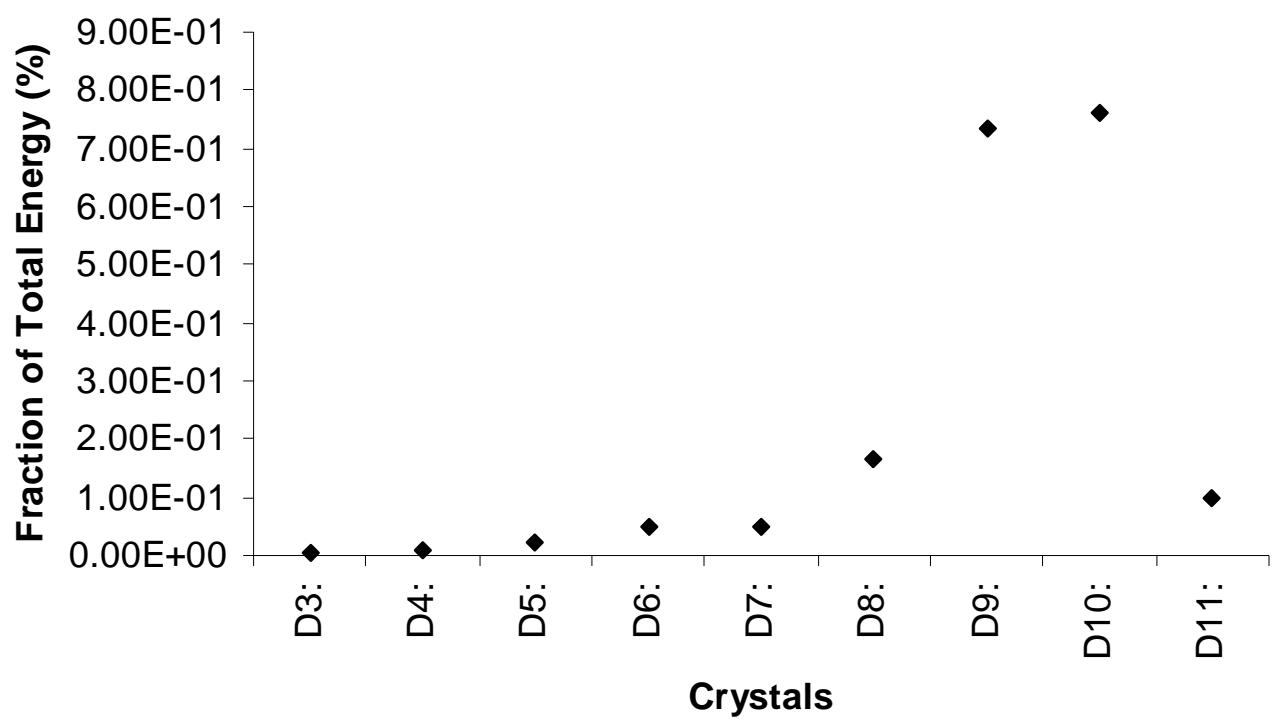

Figure III-4.22. WA of the 2,048 data from Figure Error! Reference source not found; the peaks in crystals D9 and D10 agree with the calculated frequencies of repassivation of the large transient shown in Figure III-4.21.

\section{III-4.3e WA of Data Generated Under Combinations of Corrosion Conditions}

The goal of this section was to identify the effects that various chemicals in waste storage containers have on the corrosion and upon the resulting EN data. In each run, EN data were recorded for one hour after each chemical injection before additional chemicals were added. Comparison of the EN signals and the results of WA before and after successive additions will show the effects of the interactions. The EDP percentages for all combination corrosion figures are given in Tables III-4.3 and III-4.4. 
Table III-4.3: Fractional energy contribution totals (\%); combination corrosion conditions, Run A.

\begin{tabular}{|l|c|c|c|c|}
\hline \multicolumn{1}{c|}{} & \multicolumn{5}{c|}{ Combination Corrosion Conditons, Run A } & \\
\hline & General & Post-Chloride & Post-Nitrite & Post-Nitrate \\
\hline Crystals & & & & \\
\hline D3: & $3.40 \mathrm{E}-02$ & $7.67 \mathrm{E}-02$ & $6.98 \mathrm{E}-04$ & $1.22 \mathrm{E}-03$ \\
\hline D4: & $4.93 \mathrm{E}-02$ & $1.05 \mathrm{E}-01$ & $7.39 \mathrm{E}-04$ & $1.74 \mathrm{E}-03$ \\
\hline D5: & $4.25 \mathrm{E}-02$ & $1.18 \mathrm{E}-01$ & $8.90 \mathrm{E}-04$ & $2.13 \mathrm{E}-03$ \\
\hline D6: & $2.29 \mathrm{E}-02$ & $2.23 \mathrm{E}-01$ & $1.36 \mathrm{E}-03$ & $2.83 \mathrm{E}-03$ \\
\hline D7: & $1.74 \mathrm{E}-02$ & $2.52 \mathrm{E}-01$ & $2.33 \mathrm{E}-03$ & $4.73 \mathrm{E}-03$ \\
\hline D8: & $1.83 \mathrm{E}-02$ & $2.77 \mathrm{E}-01$ & $3.36 \mathrm{E}-03$ & $7.52 \mathrm{E}-03$ \\
\hline D9: & $6.27 \mathrm{E}-03$ & $8.12 \mathrm{E}-01$ & $5.83 \mathrm{E}-03$ & $1.36 \mathrm{E}-02$ \\
\hline D10: & - & $4.85 \mathrm{E}-01$ & $1.00 \mathrm{E}-02$ & $1.66 \mathrm{E}-02$ \\
\hline D11: & - & $2.24 \mathrm{E}-01$ & $1.59 \mathrm{E}-02$ & $3.19 \mathrm{E}-02$ \\
\hline D12: & - & $4.61 \mathrm{E}+00$ & $4.90 \mathrm{E}-02$ & $9.12 \mathrm{E}-02$ \\
\hline D13: & - & $2.52 \mathrm{E}+00$ & $3.90 \mathrm{E}-01$ & $4.65 \mathrm{E}-01$ \\
\hline D14: & - & - & $6.33 \mathrm{E}-01$ & $7.02 \mathrm{E}-02$ \\
\hline D15: & - & - & - & - \\
\hline D16: & - & - & - & - \\
\hline D17: & - & - & - & - \\
\hline S: & $9.98 \mathrm{E}+01$ & $9.03 \mathrm{E}+01$ & $9.89 \mathrm{E}+01$ & $9.93 \mathrm{E}+01$ \\
\hline
\end{tabular}


Table III-4.4: Fractional energy contribution totals (\%); combination corrosion conditions, Run B.

\begin{tabular}{|c|c|c|c|c|}
\hline & \multicolumn{3}{|c|}{ Combination Corrosion Conditons, Run B } & \multirow[b]{2}{*}{ Post-Nitrite } \\
\hline & General & Post-Chloride & Post-Nitrate & \\
\hline Crystals & & & & \\
\hline D3: & 1.14E-01 & 4.59E-01 & $2.41 \mathrm{E}+00$ & $2.96 \mathrm{E}-03$ \\
\hline D4: & 1.87E-01 & 7.26E-01 & $3.27 \mathrm{E}+00$ & $5.46 \mathrm{E}-03$ \\
\hline D5: & 1.91E-01 & 8.33E-01 & $2.93 \mathrm{E}+00$ & $9.58 \mathrm{E}-03$ \\
\hline D6: & 1.24E-01 & 8.02E-01 & $1.74 \mathrm{E}+00$ & 1.61E-02 \\
\hline D7: & 8.71E-02 & 9.03E-01 & $1.06 \mathrm{E}+00$ & 3.38E-02 \\
\hline D8: & 6.55E-02 & $1.31 E+00$ & 6.24E-01 & 6.67E-02 \\
\hline D9: & 3.53E-02 & $1.95 \mathrm{E}+00$ & 7.28E-01 & 1.22E-01 \\
\hline D10: & $2.78 \mathrm{E}-02$ & $3.77 \mathrm{E}+00$ & $1.35 \mathrm{E}+00$ & $2.75 \mathrm{E}-01$ \\
\hline D11: & 2.87E-02 & $6.55 \mathrm{E}+00$ & $2.73 \mathrm{E}+00$ & 3.93E-01 \\
\hline D12: & $5.72 \mathrm{E}-02$ & $6.98 \mathrm{E}+00$ & $3.21 E+00$ & 7.32E-01 \\
\hline D13: & $2.12 \mathrm{E}+00$ & $3.69 \mathrm{E}+01$ & $2.28 \mathrm{E}+01$ & 2.36E-01 \\
\hline D14: & $6.48 \mathrm{E}-01$ & $3.84 \mathrm{E}+01$ & $1.23 E+01$ & 5.34E-01 \\
\hline D15: & - & - & - & - \\
\hline D16: & - & - & - & - \\
\hline D17: & - & - & - & - \\
\hline S: & $9.62 \mathrm{E}+01$ & 1.14E-02 & $4.36 \mathrm{E}+01$ & $9.76 \mathrm{E}+01$ \\
\hline
\end{tabular}

In combination Run A, the corrosion inducing/inhibiting chemicals were injected in the following order (as defined in Table III-4.1) first, $\mathrm{NaOH}$ (base solution); second, $\mathrm{NaCl}$; third, $\mathrm{NaNO}_{2}$; fourth, $\mathrm{NaNO}_{3}$. In combination Run $\mathrm{B}$, the chemicals were injected in the order: $\mathrm{NaOH}, \mathrm{NaCl}, \mathrm{NaNO}_{3}, \mathrm{NaNO}_{2}$. Figures III-4.23 and III-4.24 show WA analyses of data taken during the first hours of Runs A and B (combination corrosion conditions), respectively, when only sodium hydroxide was present. The EDP for Run A shows the same trend as seen in the initial pure sodium hydroxide Runs A and B (Figures III-4.8 and III-4.10, respectively): no increase in the high-frequency crystals (D3-D6). The graph for Run B (combination corrosion conditions) shows a slight increase in the high-frequency crystals. This is an indication that there is very minor general corrosion occurring. 


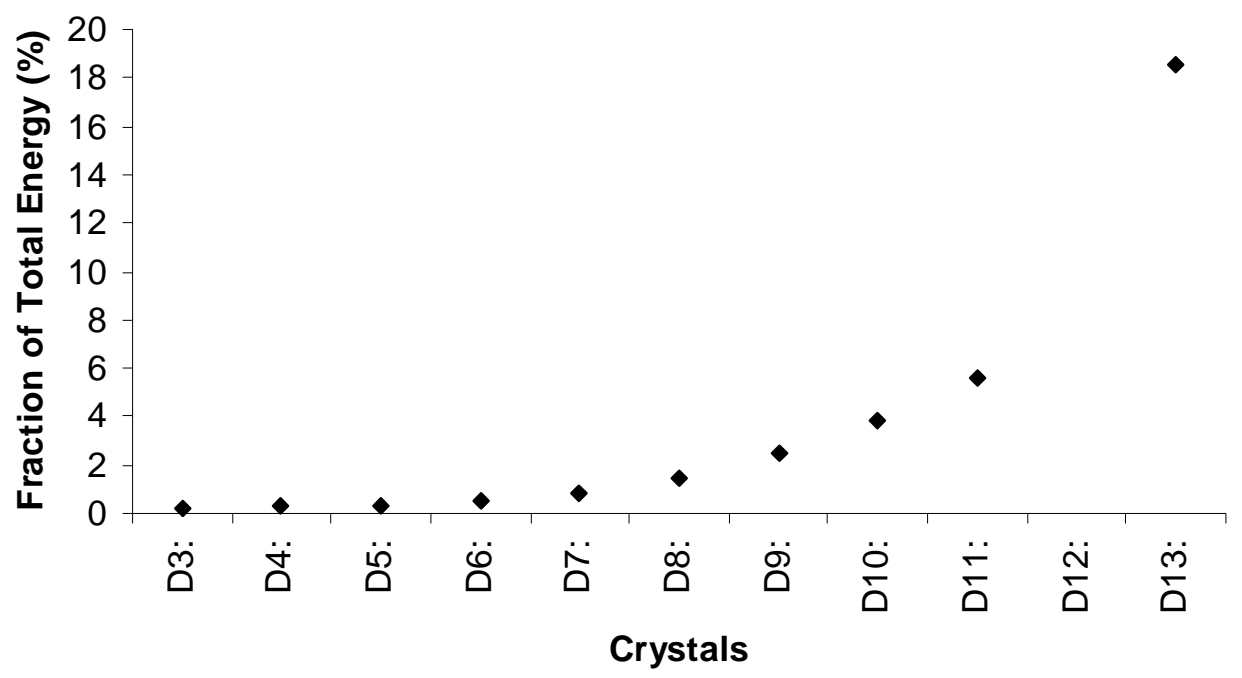

Figure III-4.23: Combination corrosion conditions, Run A; a sample of 1,024 data shows no increase in the high-frequency crystals D3-D7. This trend is the same as that seen in Figures III-4.8 and III-4.10.

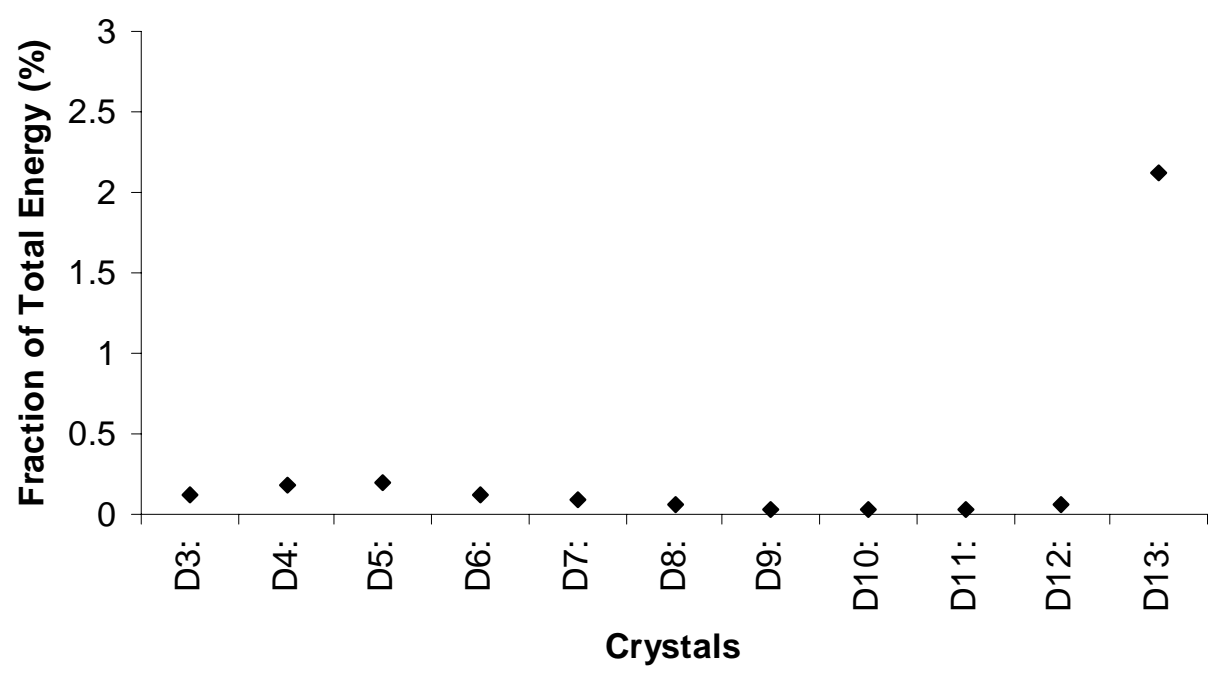

Figure III-4.24: Combination corrosion conditions, Run B; a sample of 32,768 data taken over 0.46 hours, showing a similar increase in the high-frequency crystals as seen in Figure III-4.11. 
In Run A, the chloride ion solution was the first to be added the base solution, and the results are shown in Figure III-4.19 with the results from the post-nitrate and postnitrite periods. The analyses were each done on 16,384 points taken over 0.46 hours directly after each injection. The results for the injection of chloride ions are the same as seen in pitting corrosion Runs A-E (Figures III-4.11 through III-4.18): that low-frequency crystals (D6-D11 in Figure III-4.13), corresponding to pitting corrosion, show increased contributions to the total energy.

The second step was the injection of $\mathrm{NO}_{2}{ }^{-}$ions. These ions have been shown to inhibit corrosion [14]. As expected, the energy contributions from all detail crystals drop off, and the smooth crystal energy fraction goes from 90.3 percent to 98.9 percent. The third step was the injection of $\mathrm{NO}_{3}{ }^{-}$ions - another inhibitor [15]. The trend in the EDP remains the same.

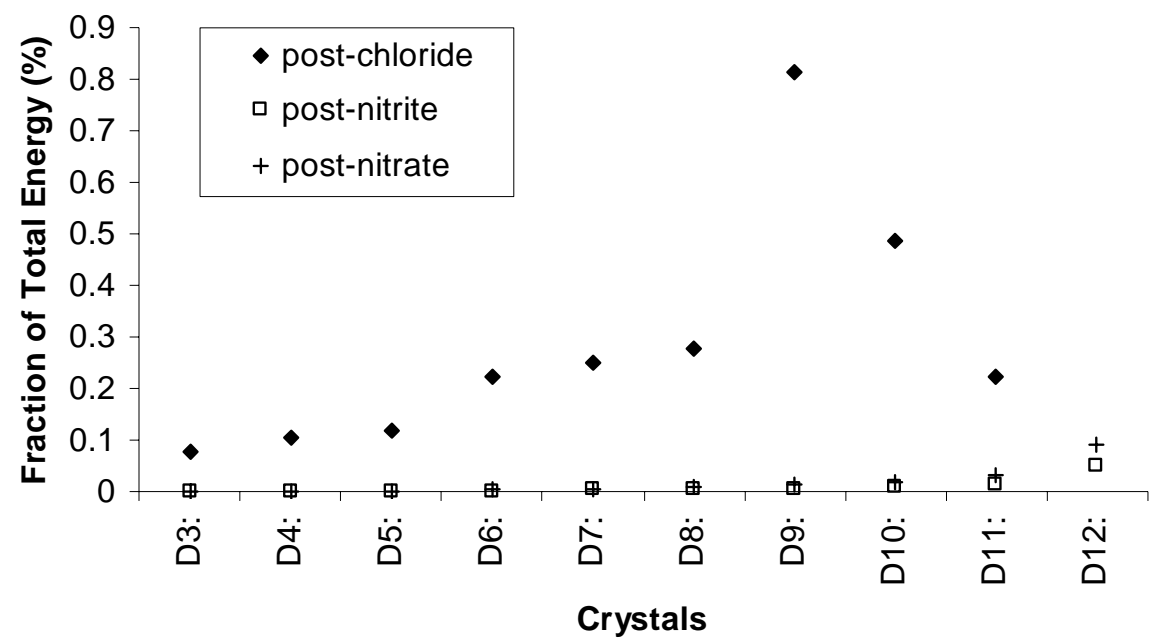

Figure III-4.25. Combination corrosion conditions, Run A; data from three 0.46 hour periods after the injection of chloride ions, nitrite ions, and nitrate ions. The low-frequency crystals (D6-D11) show increases in energy after chloride ion injection, and all detail crystals show decreased energy after the injection of the inhibitor ions.

Run B was also made under combination corrosion conditions. The first hour (with only sodium hydroxide) has already been discussed, and Figure III-4.26 shows the 
same low-frequency trend during the second hour after chloride injection. One interesting difference appears after the injection of nitrate ions. For Run B, the order of the injection of sodium nitrite and sodium nitrate was reversed from that in Run A. Here, the $\mathrm{NO}_{3}{ }^{-}$ions were injected one hour after the chloride ions and one hour before the nitrite ions.

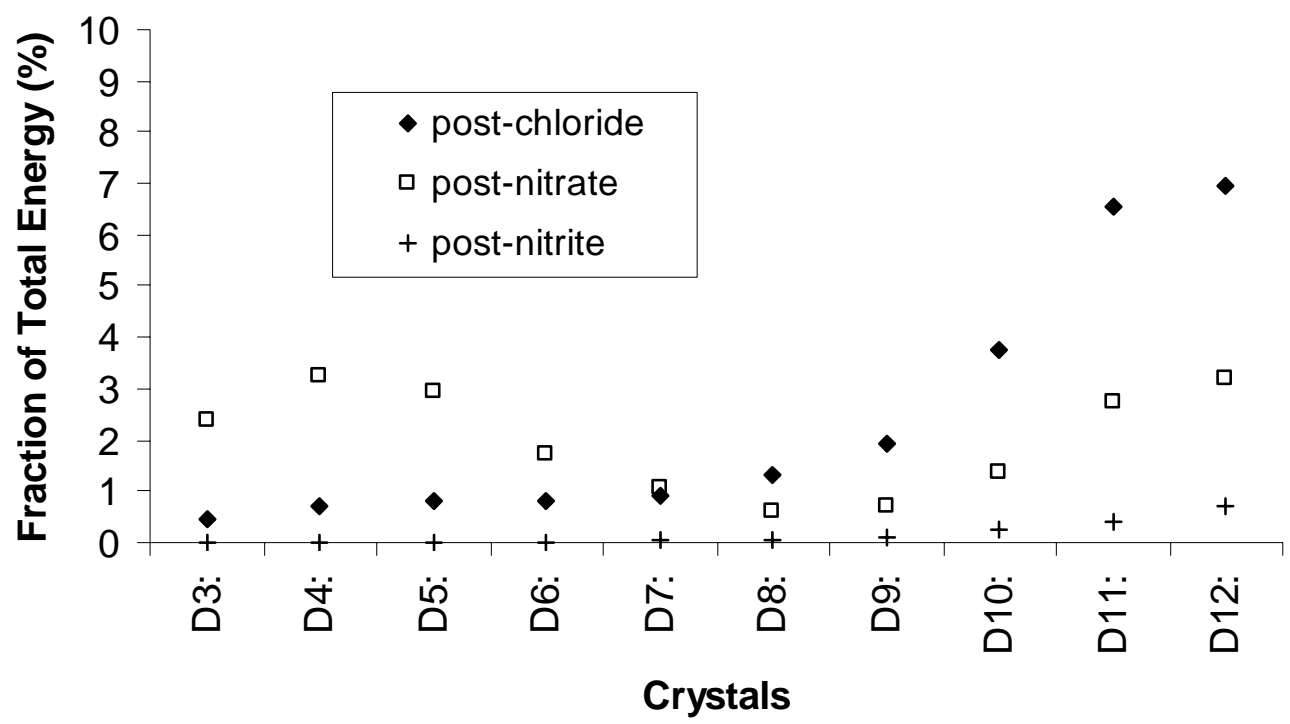

Figure III-4.26: Combination corrosion conditions, Run B; data from three 0.46 hour periods after the injection of chloride ions, nitrate ions, and nitrite ions. The low-frequency crystals show increased energy in the post-chloride period and decreased, but still significant, energy in the post-nitrate period. Also, the nitrate ions promoted general corrosion, seen in the increased energy contributions of the high-frequency crystals.

The EDP for the period after nitrate injection shows decreased energy in the lowfrequency crystals associated with pitting corrosion, but the decrease is not as drastic as seen in Run A after the injection of nitrite ions. Figure III-4.26 also shows a highfrequency increase in crystals D3-D6. This behavior is what would be expected if the nitrate ions promoted the general corrosion conditions that were seen in hour 1 of Run B (Figure III-4.24). The EDP for the period after nitrite ions shows drastic decreases in all crystal fractional energy totals, as was seen in Run A (combination corrosion conditions). 
Figures III-4.27, III-4.28, and III-4.29 show samples of the current data for the periods after chloride injection, nitrate injection, and nitrite injection, respectively. There are clear instances of overlapping pitting transients in the first figure confirming pitting corrosion. Figure III- 4.28 is on the same time and current scales, and the EN data are more random. They appear more like EN data from general corrosion, and they do not show any evidence of the large transients of pitting corrosion. Figure III-4.29 is again shown with the same scale and over the same time period as Figures III-4.27 and III-4.28. Fluctuations in the current record are almost nonexistent when compared to the other two records.

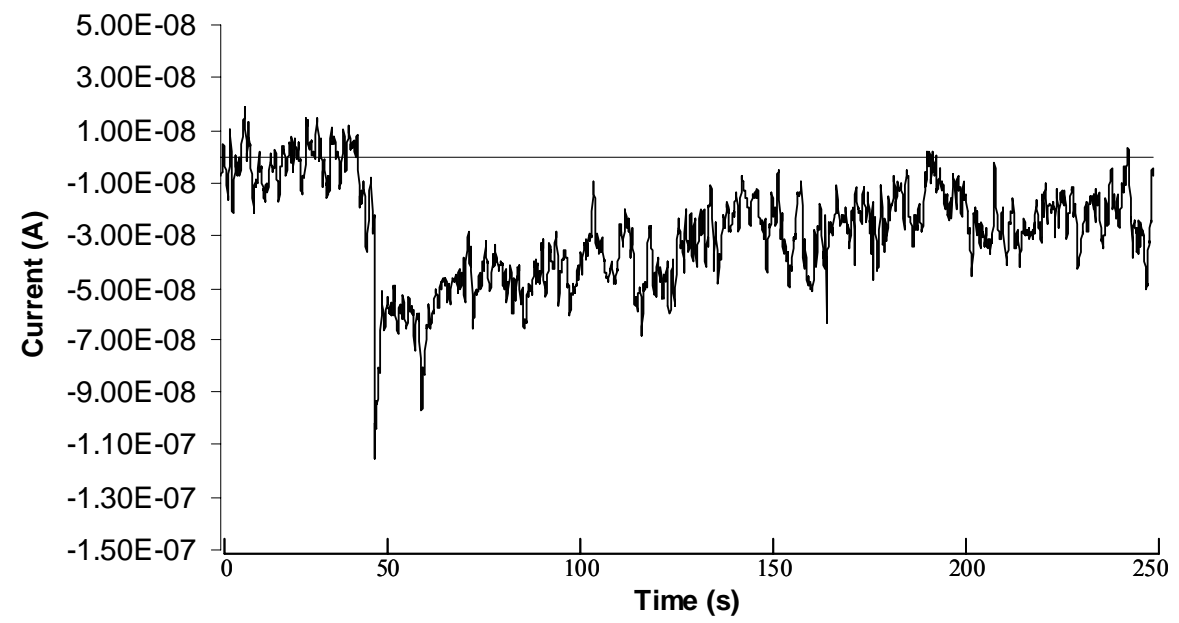

Figure III-4.27: Current data over 250 seconds of Run B; these data were collected after chloride containing solution was injected and show clear evidence of overlapping noise transients associated with pitting events and their repassivation. 


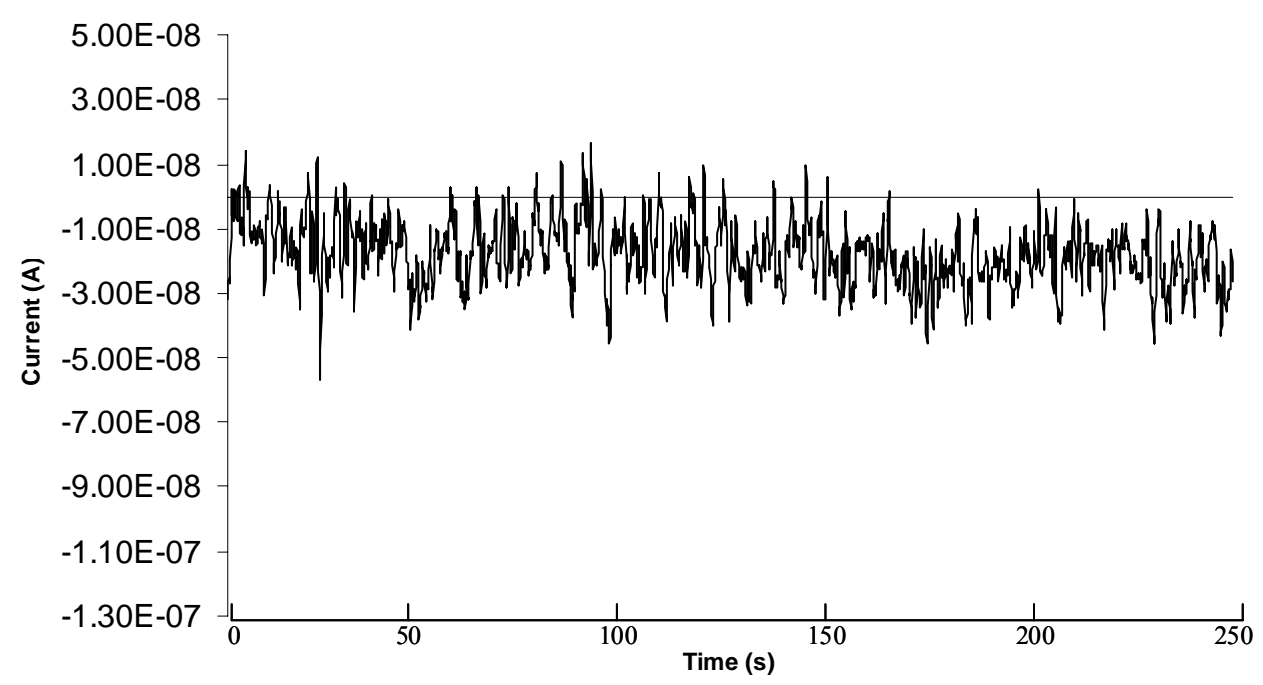

Figure III-4.28: Current data for Run B; data collected after nitrate ion containing solution was injected into the cell. Current data show no transients associated with pitting corrosion. Instead the plot has the random appearance of general corrosion data.

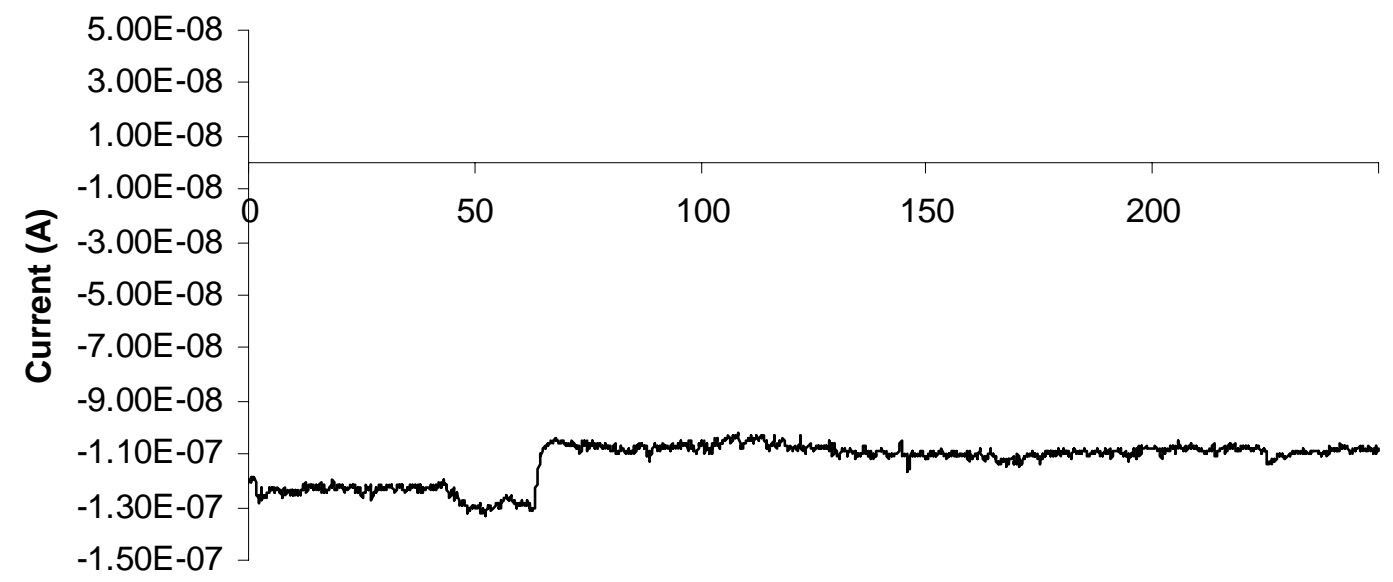

Time (s)

Figure III-4.29: Current data for Run B; data recorded after the injection of nitrite ions show a great reduction in the noise amplitude from the previous two records. 
The results of Runs A and B make it clear that while nitrate and nitrite ions both inhibit pitting corrosion in these conditions, nitrite ions are far superior in inhibiting both general corrosion and pitting corrosion, and nitrate ions will promote general corrosion.

Run C (combination corrosion conditions) included the injection of phosphate ions. The chemicals used in this run were injected in the order given in Table III-4.1, which is the same order as Run B, except for the addition of the phosphate ions after the nitrate injection and before the nitrite injection. The same general trends were seen for the injection of chloride, nitrate, and nitrite ions as discussed for Runs A and B. Figure III4.30 shows three samples of current data from the periods after nitrate, phosphate, and nitrite injection. They are all samples taken over the same amount of time, and are displayed on the same scale. From visual inspection, it is difficult to tell the three apart.

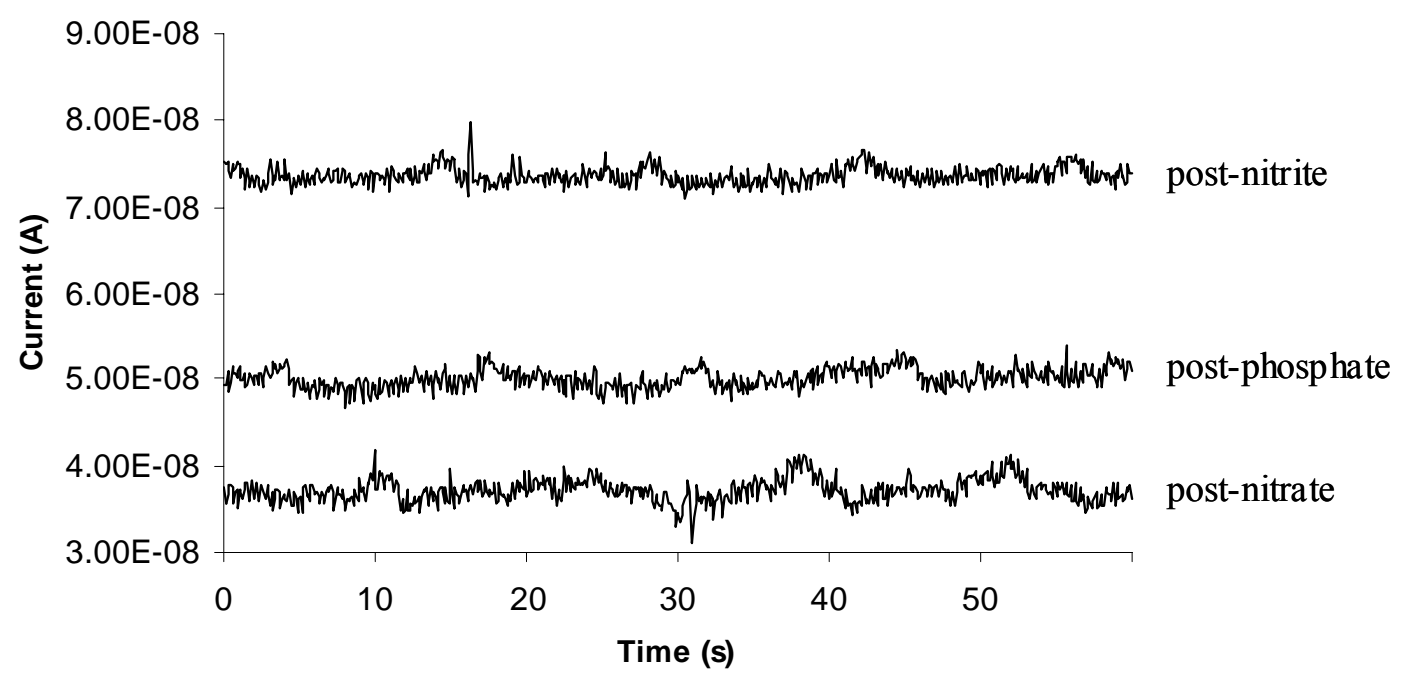

Figure III-4.30: Combination corrosion conditions, Run C; 60 seconds of current data from each of three periods: post-nitrite injection, post-phosphate injection, and post-nitrate injection. It is difficult to distinguish between the three signals by visual inspection alone.

Figure III-4.31 shows the EDPs for samples of each current record from Figure III-4.30. The WA was performed separately on 4,096 points from each record, and Table III-4.5 provides the all of the fractional energy totals for this run. The energy scale of Figure III-4.31 has been expanded for the post-phosphate numbers to show the 
differences in trend, but the scale is the same between the other two data sets. It can be seen from the differences in trend that phosphate ions promoted general corrosion (seen in the increase in energy in crystals D5-D7), and [as was seen in Runs A and B] nitrate ions were less effective at pitting corrosion inhibition than were nitrite ions (seen in the high fractions of energy in crystals D10 and D11 in the nitrate EDP, which drop off in the nitrite EDP).

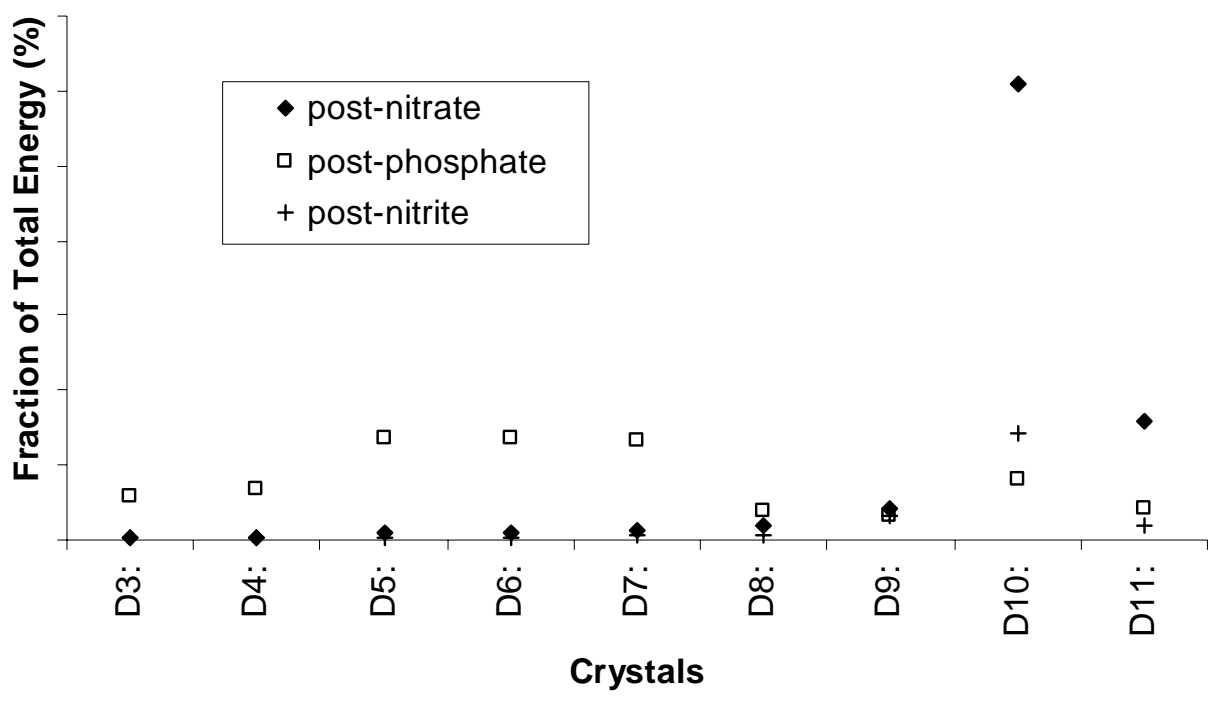

Figure III-4.31. Combination corrosion conditions, Run C; comparison of EDPs for the post-nitrate, postphosphate, and post-nitrite periods. Fractional energy scale was expanded for the post-phosphate period for comparison of trends. The nitrate ions were injected first, followed by phosphate ions which promoted general corrosion conditions that nitrite ions inhibited once injected. 
Table III-4.5. Fractional Energy Contribution (\%), combination corrosion conditions, Run C.

\begin{tabular}{|l|c|c|c|}
\hline \multirow{2}{*}{} & \multicolumn{3}{|c|}{ Combination Corrosion Conditons, Run C } \\
\hline Crystals & Post-Nitrate & Post-Phosphate & Post-Nitrite \\
\hline D3: & $1.19 \mathrm{E}-03$ & $2.91 \mathrm{E}-02$ & $1.19 \mathrm{E}-03$ \\
\hline D4: & $8.98 \mathrm{E}-04$ & $3.45 \mathrm{E}-02$ & $8.98 \mathrm{E}-04$ \\
\hline D5: & $1.26 \mathrm{E}-03$ & $6.77 \mathrm{E}-02$ & $1.26 \mathrm{E}-03$ \\
\hline D6: & $1.48 \mathrm{E}-03$ & $6.74 \mathrm{E}-02$ & $1.48 \mathrm{E}-03$ \\
\hline D7: & $2.87 \mathrm{E}-03$ & $6.70 \mathrm{E}-02$ & $2.87 \mathrm{E}-03$ \\
\hline D8: & $3.99 \mathrm{E}-03$ & $1.91 \mathrm{E}-02$ & $3.99 \mathrm{E}-03$ \\
\hline D9: & $1.59 \mathrm{E}-02$ & $1.66 \mathrm{E}-02$ & $1.59 \mathrm{E}-02$ \\
\hline D10: & $7.13 \mathrm{E}-02$ & $3.99 \mathrm{E}-02$ & $7.13 \mathrm{E}-02$ \\
\hline D11: & $9.03 \mathrm{E}-03$ & $2.03 \mathrm{E}-02$ & $9.03 \mathrm{E}-03$ \\
\hline D12: & - & - & - \\
\hline D13: & - & - & - \\
\hline D14: & - & - & - \\
\hline D15: & - & - & - \\
\hline D16: & - & - & - \\
\hline D17: & - & $1.60 \mathrm{E}+03$ & $9.99 \mathrm{E}+01$ \\
\hline S: & $9.99 \mathrm{E}+01$ & & \\
\hline
\end{tabular}

The results of Run D (combination corrosion conditions) further show the role of nitrate ions. Figure III-4.32 shows samples of the current record from the first three hours of the run. Hour one was under pure sodium hydroxide with no added chemicals; nitrate ions were injected at the beginning of hour two, and chloride ions were injected at the beginning of hour three. 


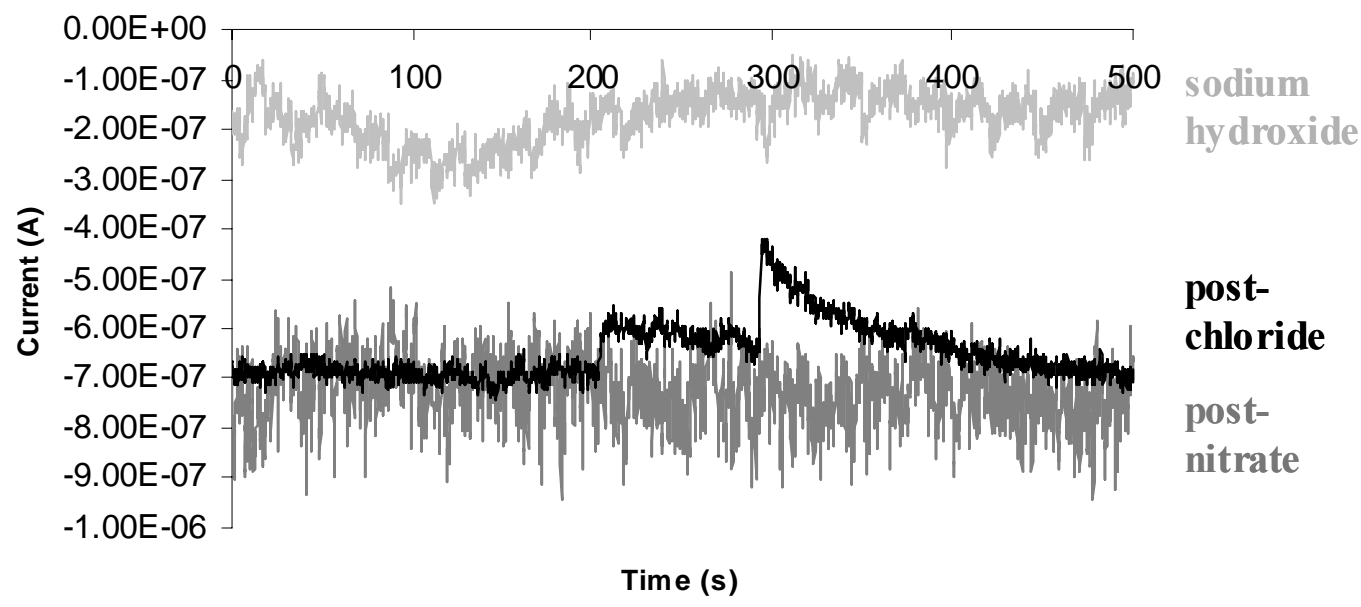

Figure III-4.32: Combination corrosion conditions, Run D; current records over 500 seconds for periods of pure sodium hydroxide, post-nitrate, and post-chloride conditions. Increased magnitude of the post-nitrate period indicates increased general corrosion noise.

Figure III-4.33 shows the EDPs for the current records in Figure III-4.32, and Table III-4.6 provides the fractional energy totals for Run D. The scale of Figure III-4.33 has been expanded for the post-nitrate period to show the difference in its trend versus the other two data sets. It is clear from the increased magnitude of noise fluctuations in the current record and the increase in energy contribution from the high-frequency crystals (D3-D7) in the EDP for the post-nitrate period, that nitrate ions promoted general corrosion conditions. The lack of significant energy increase in the low-frequency crystals associated with pitting corrosion (generally, D8-D11) in the post-chloride data set shows the pitting inhibition effect of nitrate ions. 


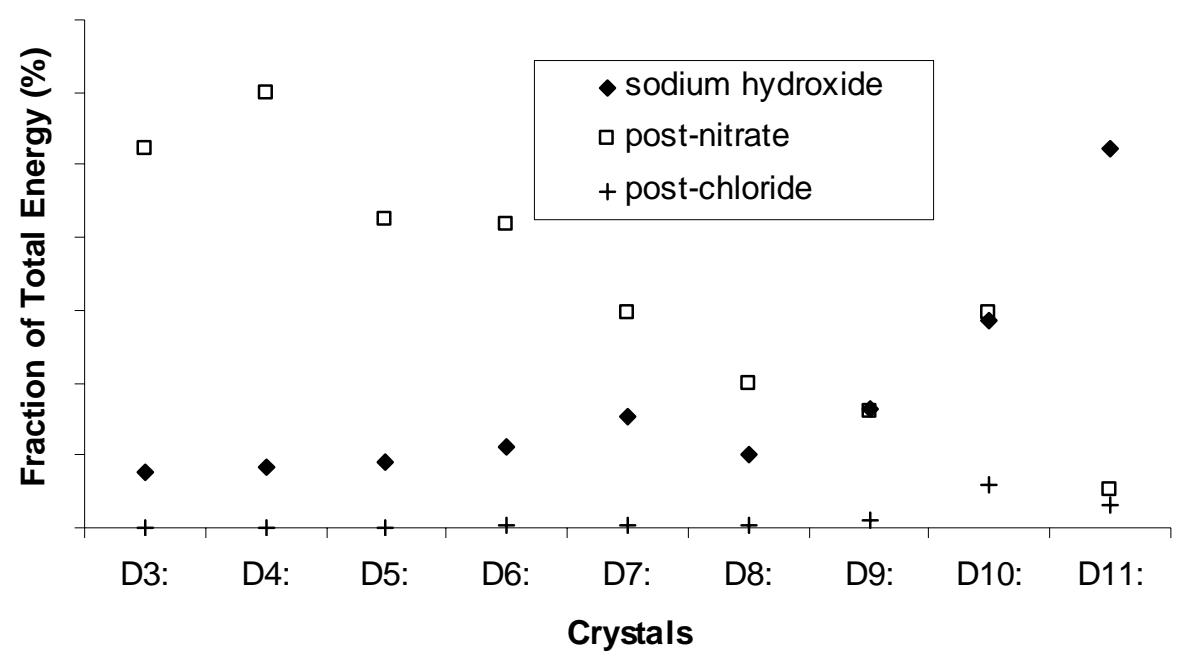

Figure III-4.33. Combination corrosion conditions, Run D; EDPs on an expanded scale showing the trend changes between the three periods. The increased coefficient values for crystals D3-D7 of the post-nitrate period confirm that the nitrate ions promoted general corrosion conditions.

Table III-4.6. Fractional Energy Contribution (\%); combination corrosion conditions, Run D.

\begin{tabular}{|l|c|c|c|}
\hline \multirow{2}{*}{} & \multicolumn{3}{|c|}{ Combination Corrosion Conditons, Run D } \\
\cline { 2 - 4 } & Sodium Hydroxide & Post-Nitrate & Post-Chloride \\
\hline Crystals & & & \\
\hline D3: & $1.39 \mathrm{E}-01$ & $6.54 \mathrm{E}-02$ & $4.63 \mathrm{E}-03$ \\
\hline D4: & $1.76 \mathrm{E}-01$ & $7.50 \mathrm{E}-02$ & $7.89 \mathrm{E}-03$ \\
\hline D5: & $1.62 \mathrm{E}-01$ & $5.29 \mathrm{E}-02$ & $7.19 \mathrm{E}-03$ \\
\hline D6: & $2.32 \mathrm{E}-01$ & $5.22 \mathrm{E}-02$ & $1.08 \mathrm{E}-02$ \\
\hline D7: & $3.11 \mathrm{E}-01$ & $3.70 \mathrm{E}-02$ & $1.62 \mathrm{E}-02$ \\
\hline D8: & $2.50 \mathrm{E}-01$ & $2.50 \mathrm{E}-02$ & $1.80 \mathrm{E}-02$ \\
\hline D9: & $1.22 \mathrm{E}-01$ & $2.02 \mathrm{E}-02$ & $4.83 \mathrm{E}-02$ \\
\hline D10: & $3.68 \mathrm{E}+00$ & $3.69 \mathrm{E}-02$ & $2.90 \mathrm{E}-01$ \\
\hline D11: & $5.40 \mathrm{E}-01$ & $6.57 \mathrm{E}-03$ & $1.56 \mathrm{E}-01$ \\
\hline D12: & - & - & - \\
\hline D13: & - & - & - \\
\hline D14: & - & - & - \\
\hline D15: & - & - & - \\
\hline D16: & - & - & - \\
\hline D17: & - & $9.96 \mathrm{E}+01$ & $9.94 \mathrm{E}+01$ \\
\hline S: & $9.42 \mathrm{E}+01$ & \multicolumn{2}{|c}{} \\
\hline
\end{tabular}




\section{WA During Corrosion Character Change}

Figure III-4.34 shows a sample of current data (collected before, during, and after the injection of chloride ions) from Run B (combination corrosion conditions). The series contains 8,192 points taken over 819.2 seconds. The injection of chloride ions occurred at approximately 484 seconds.

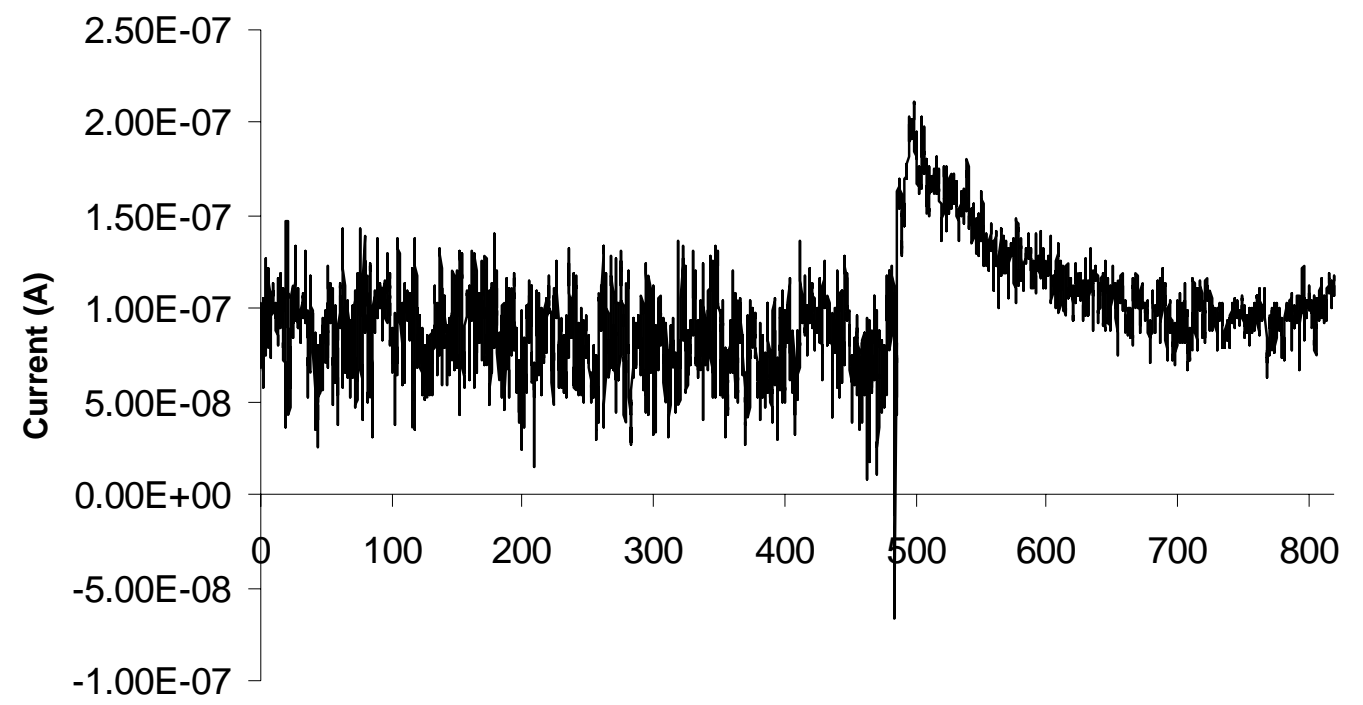

Time (s)

Figure III-4.34. Current noise data in combination corrosion conditions, Run B; general corrosion was the prevailing type of corrosion until 484 seconds when chloride ions were introduced, inducing pitting corrosion.

The EPDs for this section of current data are able to show two distinct types of corrosion, and all the crystal energy totals are given in Table III-4.7. Figure III-4.35 shows the EDP for 204.8 seconds of signal from Run B leading up to the injection of the ions, while Figure III-4.36 shows the EDP for 204.8 seconds after injection. The first graph clearly shows the contribution from the high-frequency crystals, and the second shows the contribution from the low-frequency crystals. It is logical that the WA of the entire time period (819.2 seconds) shows contributions from both, as seen in Figure III-4.37. 
Table III-4.7. Fractional Energy Contribution (\%); combination corrosion conditions, Run B, before and after injection of chloride ions (series 1 and 2, respectively), and during entire process (series 3 ).

\begin{tabular}{|c|c|c|c|}
\hline & \multicolumn{3}{|c|}{ Corrosion Character Change } \\
\hline & \multicolumn{3}{|c|}{ Combination Conditions, Run B } \\
\hline Crystals & series 1 & series 2 & series 3 \\
\hline D3: & 3.21E-01 & 2.75E-02 & 1.90E-01 \\
\hline D4: & 5.91E-01 & 6.44E-02 & 3.30E-01 \\
\hline D5: & 5.51E-01 & 9.90E-02 & 3.85E-01 \\
\hline D6: & $3.52 E-01$ & 4.27E-02 & 1.92E-01 \\
\hline D7: & 3.57E-01 & 1.04E-01 & 1.35E-01 \\
\hline D8: & 2.13E-01 & 5.14E-01 & 2.16E-01 \\
\hline D9: & 8.92E-02 & 3.98E-01 & 3.69E-01 \\
\hline D10: & - & - & $6.28 \mathrm{E}-01$ \\
\hline D11: & - & - & 1.07E+00 \\
\hline D12: & - & - & $1.06 \mathrm{E}+00$ \\
\hline D13: & - & - & - \\
\hline D14: & - & - & - \\
\hline D15: & - & - & - \\
\hline D16: & - & - & - \\
\hline D17: & - & - & - \\
\hline S: & $9.74 \mathrm{E}+01$ & $9.87 E+01$ & $9.53 E+01$ \\
\hline
\end{tabular}

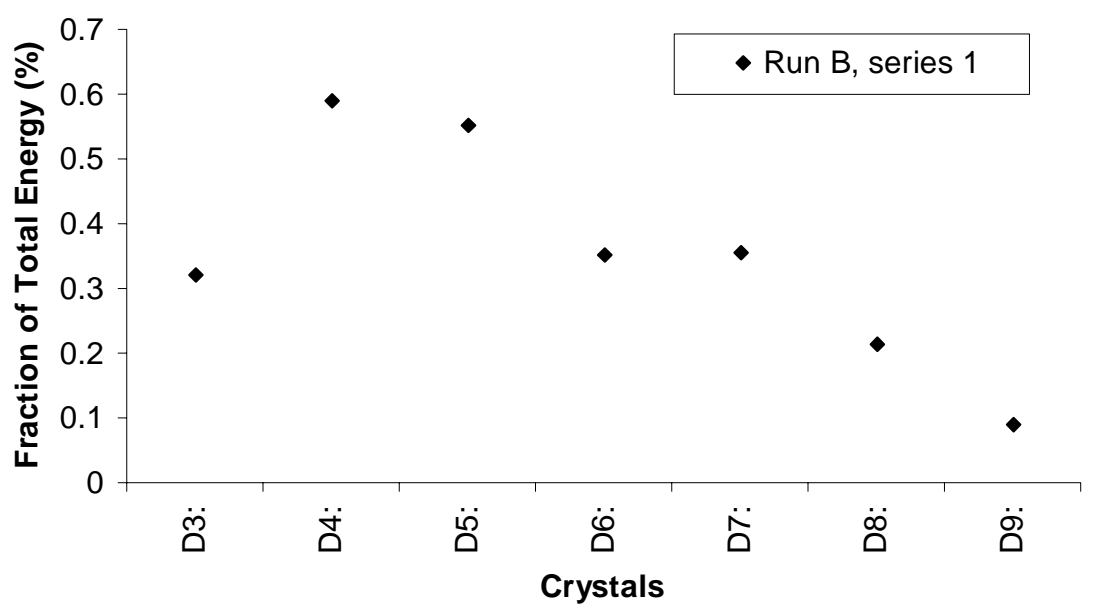


Figure III-4.35. Combination corrosion conditions, Run B series 1; EDP of 204.8 seconds prior to injection of chloride ions. Increases in contributions from high-frequency crystals are indicative of general corrosion.

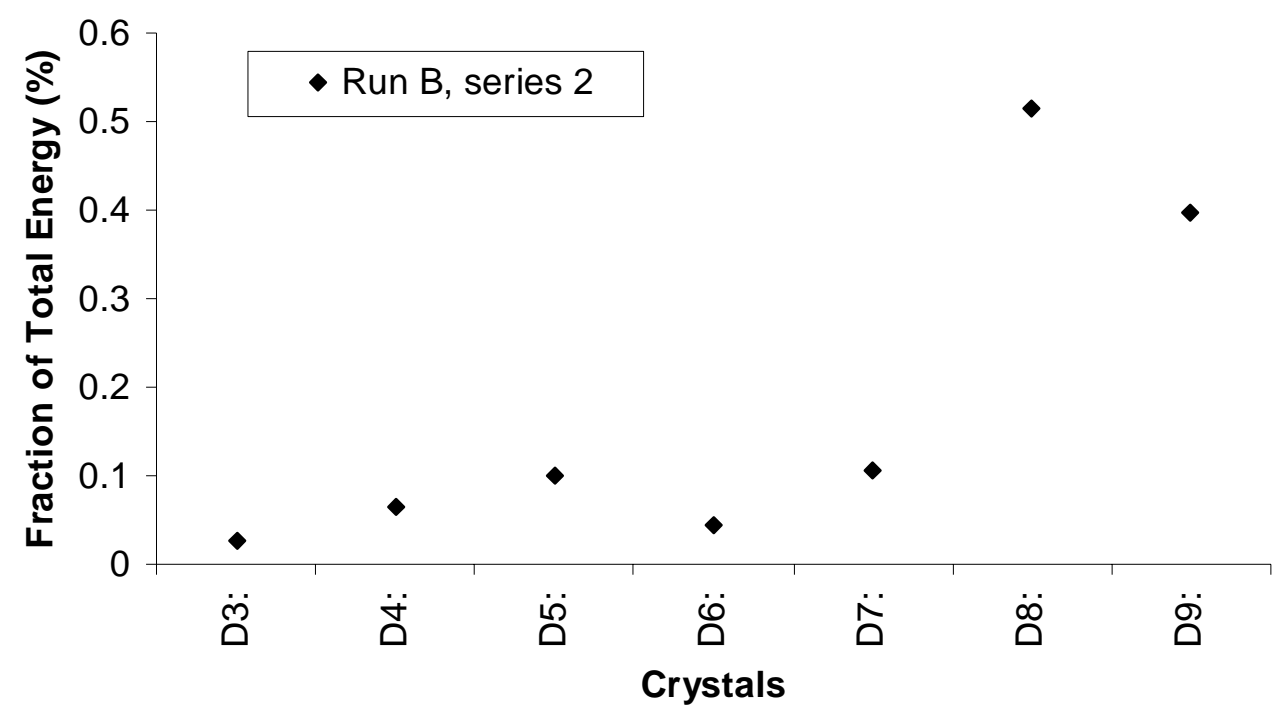

Figure III-4.36. Combination corrosion conditions, Run B series 2; EDP of 204.8 seconds after injection of chloride ions. Increase in contributions from low-frequency crystals is indicative of pitting corrosion. 


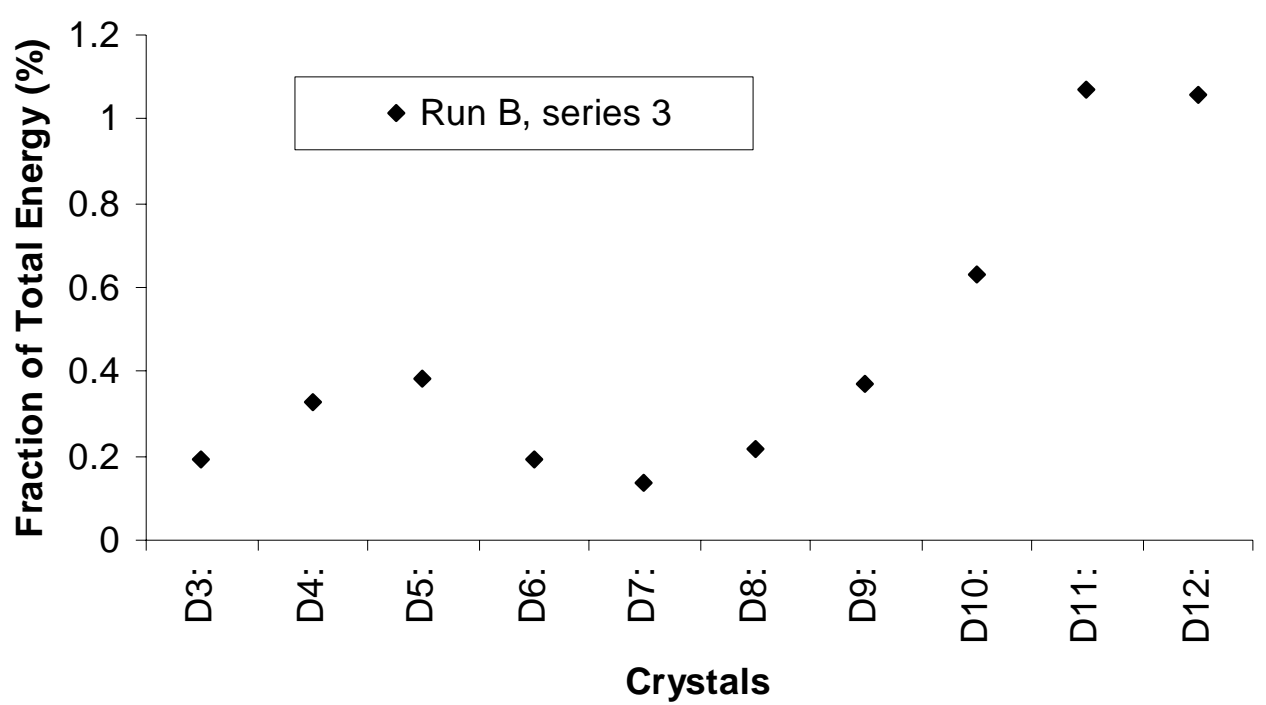

Figure III-4.37. Combination corrosion conditions, Run B series 3; EDP of 8,192 points over 13.65 minutes, shows contributions from both general corrosion and pitting corrosion, as expected.

If the time of injection isn't known, WA provides a means to determine it. Figure III-4.38 shows the low frequency crystals, D3 and D4, of the WA of this period of Run B. These crystals both show a marked change at the time of injection. The high amplitude of the wavelet coefficients prior to the moment of injection indicate that the high-frequency wavelet functions matched strongly with the current data. After injection, the magnitudes of the high-frequency coefficients are reduced indicating that they no longer match well. Thus, by examining the time domain depiction of the wavelet coefficients, or by calculating the standard deviation of these coefficients over time, it is possible to distinguish the type, and onset or termination of the corrosion. 


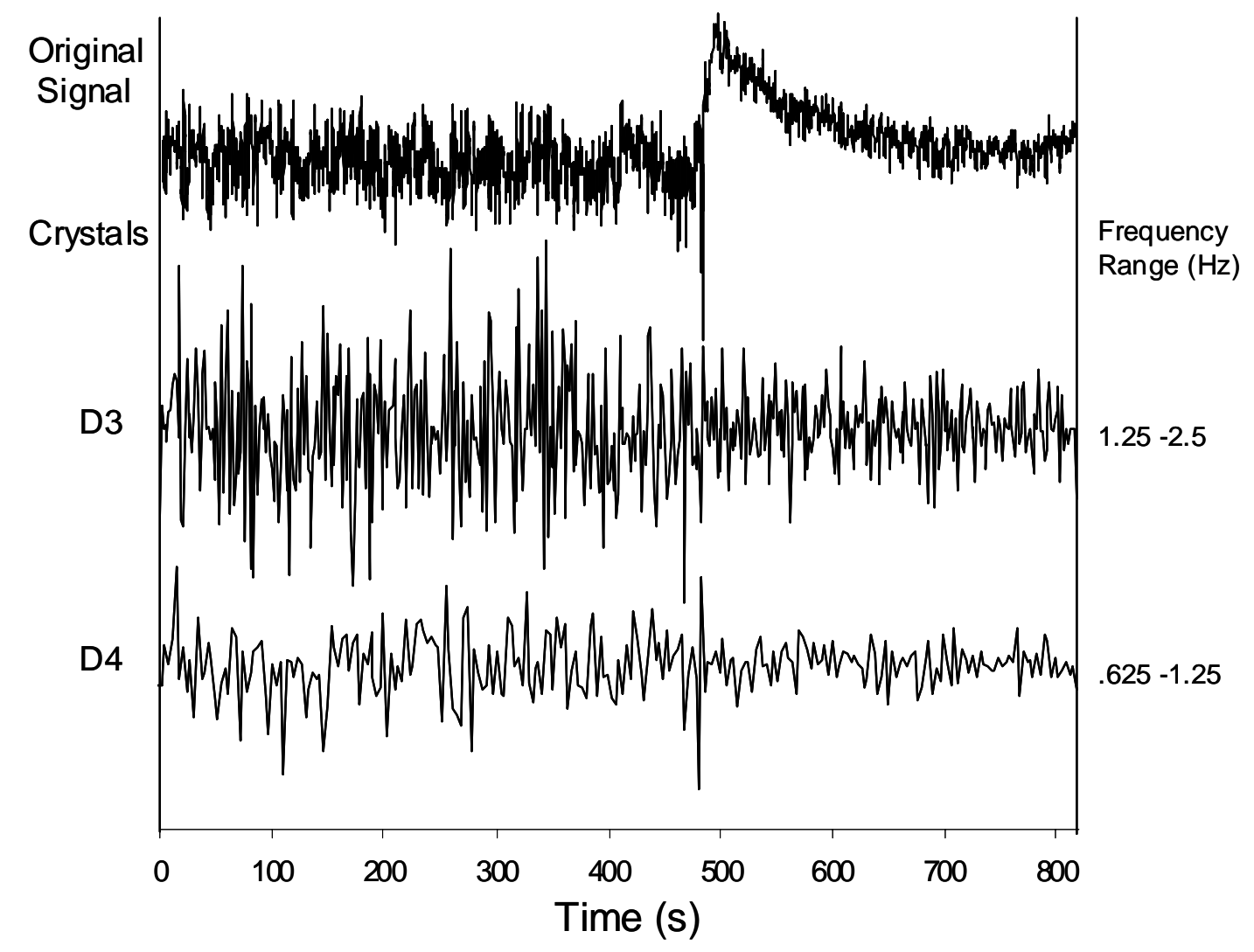

Figure III-4.38. Combination corrosion conditions, Run B; illustration of the original signal versus two high-frequency crystals (D3 and D4) shows large magnitudes in the crystal coefficients for the time leading up to the injection of chloride ions. After injection, the coefficients decrease in magnitude indicating a switch from the domination of high-frequency noise, to lower-frequencies, which is an indication of the dominance of pitting after injection.

\section{III-4.3f WA of Simulated Data}

\section{WA of Random Noise Simulations}

Figures III-4.39 and III-4.40 show the EDPs of random noise simulations where no rules were applied; only the current density values changed with time. As expected, 
there is an increase in the high-frequency crystals (D1-D5) in both figures. Since these data are simulated, there is no aliasing, and all crystals are shown. Figure III-4.39 was from a simulation of 2,048 data. Figure III-4.40 is from a simulation of 4,096 data.

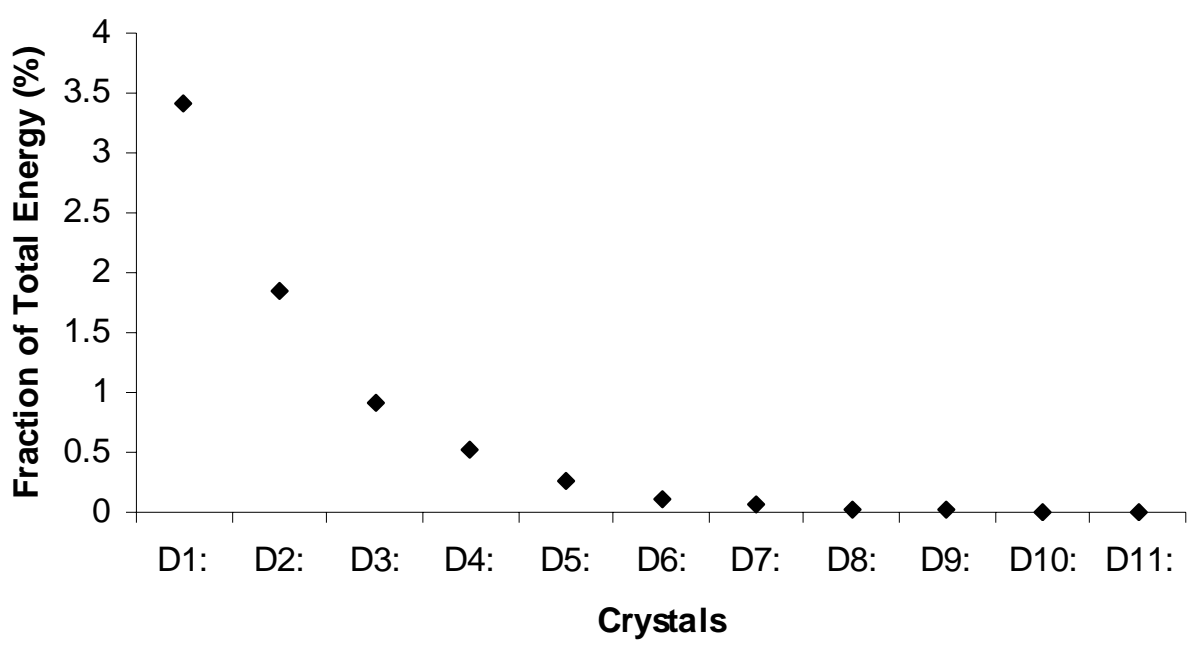

Figure III-4.39. Random noise simulation, Run A.

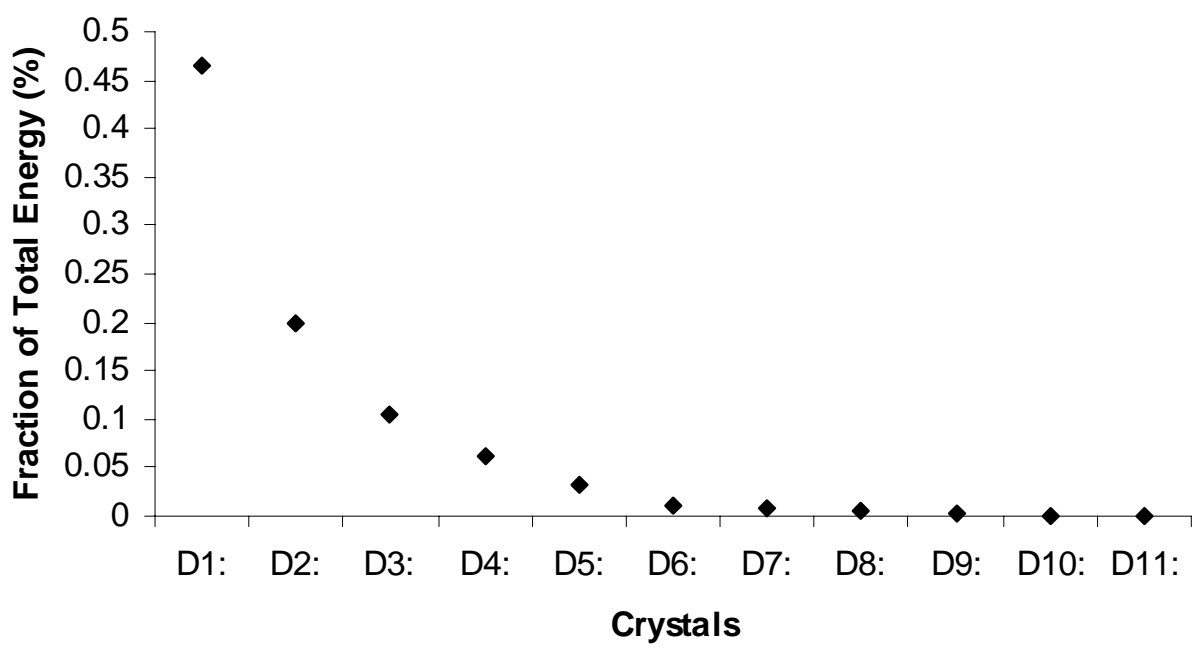

Figure III-4.40. Random noise simulation, Run B. 


\section{WA of Rule-Based Noise Simulations}

The rule-based simulation includes those rules discussed in section III-4.1 and has nine main variables that control the interaction of sites. There is a variable called "fCathFrac" that defines how much of the current generated at each anodic site must be consumed by local cathodes for the anode to survive (the remaining fraction is assumed to flow to the counter electrode). The variables "fAProb" and "fCProb" are the probabilities at the start of the program that a site in the array is assigned an anodic or cathodic character, respectively. "fImpProb" is the increased chance that neutral sites have of being assigned as cathodic when they are adjacent to an anodic site. These three probabilities are only used to set up the initial arrangement of sites, and are only used once per simulation.

The next set of variables governs the probabilities of sites switching from neutral character to anodic or cathodic during the simulation. "cfAIncrease" defines the probability that a neutral site that is not already adjacent to an anodic site becomes anodic during each cycle of the simulation. "cfCDist0", "cfCDist3", and "cfCDist1" define the probability that neutral sites become cathodic when separated from nearby anodic sites by average distances of greater than three sites, equal to three sites, and equal to one site, respectively. The probability for sites with an average distance between three and one is scaled using "cfCDist3" and "cfCDist1" as limits. The final variable of interest is "cfCRand" which governs that random chance that a cathodic site will die. This accounts for the reasons for cathodic site death not otherwise covered in this simulation. The other governing rules are not mentioned here because they are not dependent on variable values, so they affect all simulation runs equally.

The variables for simulation Runs A through E and the energy contributions for all crystals from the WA of those runs are given in Tables III-4.8 and III-4.9, respectively. All simulations runs were performed over 6.83 minutes (4,096 data). For Run A, the variables for the birth and death of cathodic and anodic sites were given small starting values (on the order of tenths of a percent) and the fraction of anodic current to be consumed at nearby cathodic sites for the pit to survive was set to 75 percent. Figures III-4.41 and III-4.42show the results of Run A. The first figure shows the current data 
generated and the second shows the results of the WA. It is clear from both figures that the data are not simply randomly dependent. There is no high-frequency tail as seen in Figures III-4.39 and III-4.40, but there are two peaks in crystals D4-D6 and D7-D10 showing a shift toward lower-frequency dependence.

Table III-4.8. Variable values for simulation Runs A-E.

\begin{tabular}{|c|c|c|c|c|c|}
\hline & Simulation & Runs & & & \\
\hline Variables & A & $\mathrm{B}$ & C & $D$ & $\mathrm{E}$ \\
\hline fCathFrac & 0.75 & 0.25 & 0.25 & 0.25 & 0.05 \\
\hline fAProb & 0.1 & 0.1 & 0.1 & 0.1 & 0.1 \\
\hline fCProb & 0.04 & 0.04 & 0.04 & 0.04 & 0.04 \\
\hline pProb & 0.05 & 0.05 & 0.05 & 0.05 & 0.05 \\
\hline ease & 0.001 & 0.001 & 0.001 & 0.001 & 0.001 \\
\hline & 0.001 & 0.001 & 0.001 & 0.001 & 0.001 \\
\hline & 0.006 & 0.006 & 0.006 & 0.001 & 0.006 \\
\hline cfCDist1 & 0.018 & 0.018 & 0.018 & 0.003 & 0.018 \\
\hline cfCRand & 0.03 & 0.03 & 0.08 & 0.03 & 0.03 \\
\hline
\end{tabular}

Table III-4.9. Fraction of Total Energy (\%) in crystals of simulation Runs A-E.

\begin{tabular}{|c|c|c|c|c|c|}
\cline { 2 - 6 } \multicolumn{1}{c|}{} & Simulation Runs \\
\hline Crystals & A & B & C & D & E \\
\hline D1: & $8.62 \mathrm{E}-02$ & $1.16 \mathrm{E}-01$ & $9.02 \mathrm{E}-01$ & $9.18 \mathrm{E}+00$ & $4.69 \mathrm{E}-01$ \\
D2: & $9.81 \mathrm{E}-02$ & $1.31 \mathrm{E}-01$ & $8.50 \mathrm{E}-01$ & $6.46 \mathrm{E}+00$ & $6.02 \mathrm{E}-01$ \\
D3: & $1.78 \mathrm{E}-01$ & $2.14 \mathrm{E}-01$ & $1.45 \mathrm{E}+00$ & $6.22 \mathrm{E}+00$ & $1.02 \mathrm{E}+00$ \\
D4: & $3.82 \mathrm{E}-01$ & $3.49 \mathrm{E}-01$ & $2.36 \mathrm{E}+00$ & $7.38 \mathrm{E}+00$ & $1.51 \mathrm{E}+00$ \\
D5: & $4.66 \mathrm{E}-01$ & $8.26 \mathrm{E}-01$ & $2.47 \mathrm{E}+00$ & $1.59 \mathrm{E}+01$ & $3.19 \mathrm{E}+00$ \\
D6: & $3.64 \mathrm{E}-01$ & $9.41 \mathrm{E}-01$ & $2.63 \mathrm{E}+00$ & $1.61 \mathrm{E}+01$ & $4.45 \mathrm{E}+00$ \\
D7: & $4.89 \mathrm{E}-01$ & $9.13 \mathrm{E}-01$ & $2.67 \mathrm{E}+00$ & $1.01 \mathrm{E}+01$ & $6.54 \mathrm{E}+00$ \\
D8: & $9.62 \mathrm{E}-01$ & $2.01 \mathrm{E}+00$ & $8.43 \mathrm{E}-01$ & $7.74 \mathrm{E}+00$ & $3.64 \mathrm{E}+00$ \\
D9: & $6.54 \mathrm{E}-01$ & $8.47 \mathrm{E}-01$ & $7.54 \mathrm{E}-01$ & $7.51 \mathrm{E}+00$ & $1.05 \mathrm{E}+01$ \\
D10: & $3.48 \mathrm{E}-01$ & $2.65 \mathrm{E}+00$ & $5.94 \mathrm{E}-01$ & $3.03 \mathrm{E}+00$ & $1.37 \mathrm{E}+01$ \\
D11: & $8.17 \mathrm{E}-02$ & $5.20 \mathrm{E}-01$ & $2.00 \mathrm{E}-01$ & $6.08 \mathrm{E}-01$ & $8.02 \mathrm{E}+00$ \\
D12: & - & - & - & - & - \\
D13: & - & - & - & - & - \\
D14: & - & - & - & - & - \\
D15: & - & - & - & - & - \\
D16: & - & - & - & - & - \\
D17: & - & - & - & - & - \\
S: & $9.59 \mathrm{E}+01$ & $9.05 \mathrm{E}+01$ & $8.43 \mathrm{E}+01$ & $9.76 \mathrm{E}+00$ & $4.64 \mathrm{E}+01$ \\
\hline
\end{tabular}




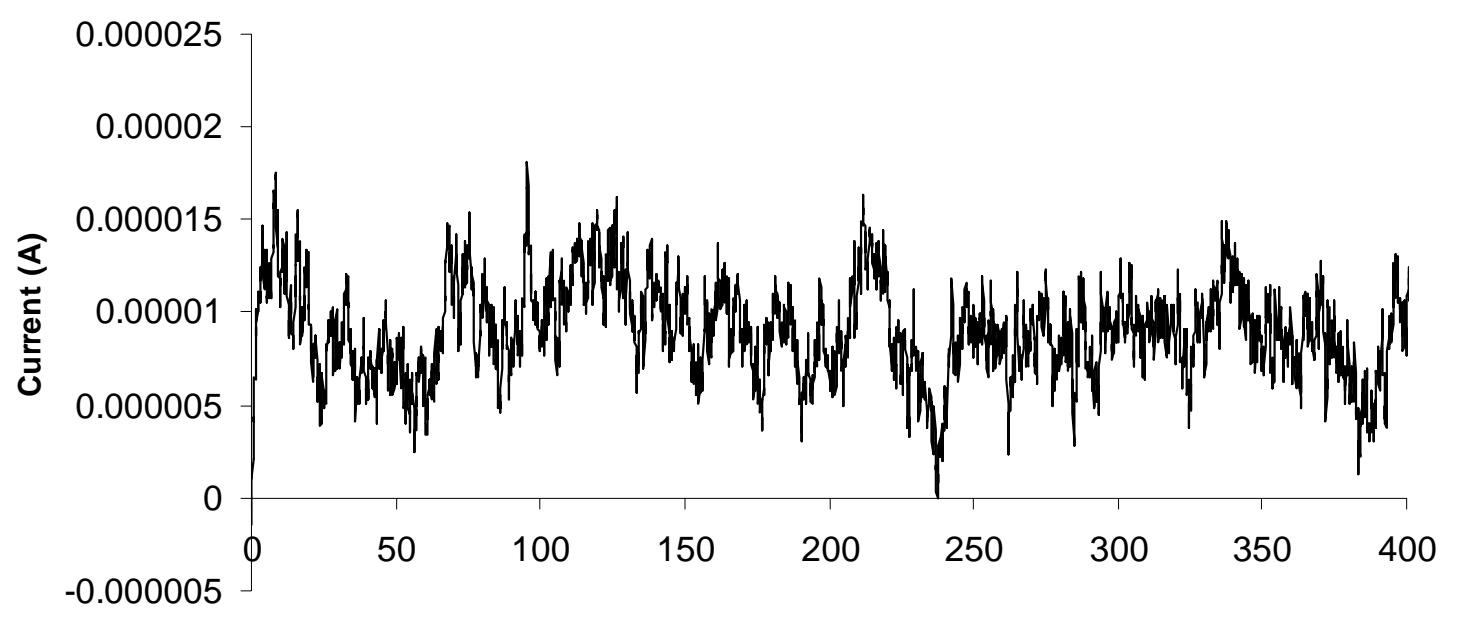

Time (s)

Figure III-4.41. Rule-based noise simulation, Run A; Current noise data over 4,096 points. Peaks and troughs in data correspond to birth and death of pits.

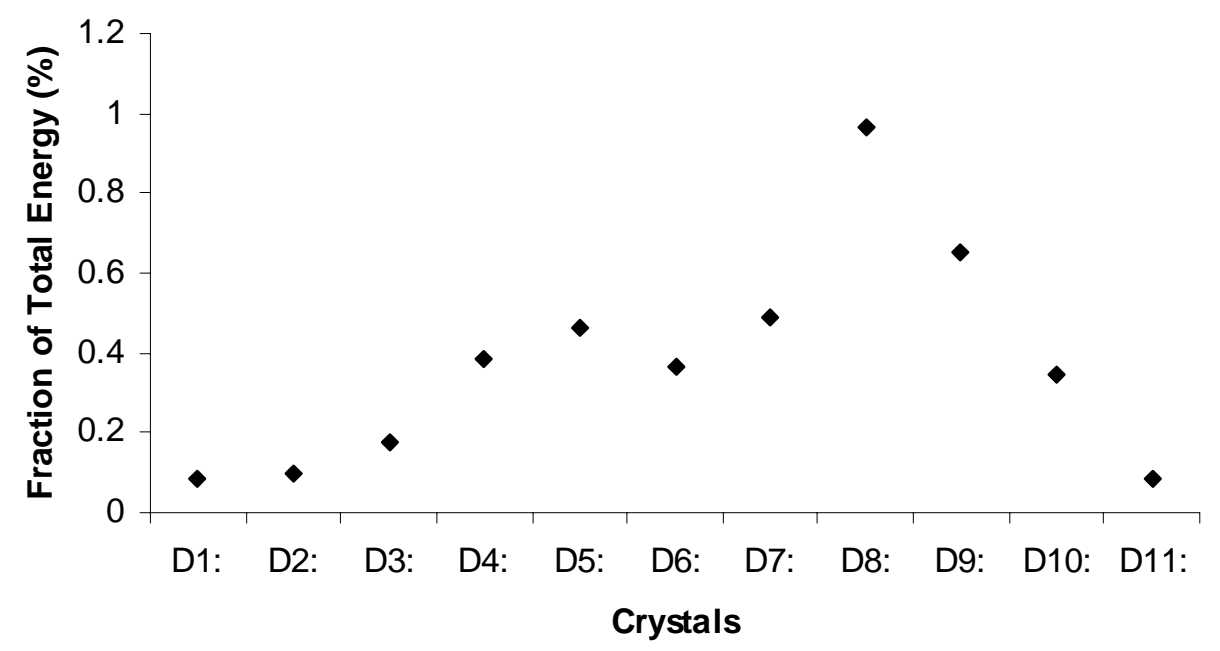


Figure III-4.42. Rule-based noise simulation, Run A; WA of current data shows no increase in the highestfrequency crystals, but, instead, increases in crystals D4-D6 and D7-D10.

These results show that the rules provided longer-range order to the current data. The next step was to see if the rules could provide data that looked similar, with respect to both the current data and the WA, to that of pitting data collected experimentally. The simulation was run repeatedly while varying the birth/death probabilities and the fraction of anodic current consumed by local cathodes across a wide range of values. The final arrangement of sites, the current data, the number of sites born and killed over time, and the WA of the current data were all examined to determine which rules were governing the interaction of the sites. It was found that when the simulation variables were tweaked such that all rules could affect the arrangement and lifetime of the sites, the resultant WA appeared very similar to that of the experimental pitting data.

Figures III-4.43 through III-4.54 show the results from simulation Runs B through E. Each run of the simulation highlights different sets of variable values. Figure III-4.43 shows the initial arrangement of sites for Run B. Since the variables governing the initial setup weren't altered from run to run, this figure is representative of all runs. Figure III4.44 shows the final arrangement of sites for Run B. In this run, the variables were set such that all rules were in effect, so it is a good standard for comparison for the other runs. 


\begin{tabular}{|c|c|c|c|c|c|c|c|c|c|c|c|c|c|c|c|c|c|c|c|}
\hline RowsiCols & 1 & & & & 5 & & & & & 10 & & & & 15 & & & & & 20 \\
\hline 1 & & & & & & & & & & & & & & & & & & & \\
\hline & & & & & & & C & A & C & & A & & & & & & & & \\
\hline & & & & & & & & & & & & & & & & & A & & A \\
\hline & & & & & A & & & & & & A & & & & & & & & \\
\hline 5 & & & & & & C & & & & & & & & & & & & & \\
\hline & & & & & A & C & & & & C & & & & A & & & & & \\
\hline & & & & & & & & & & & & C & & & & & & & \\
\hline & & C & & & & & & & & & A & & & & C & C & & & \\
\hline & & & & & & & & C & & & & & & C & & & & & \\
\hline 10 & & A & & & & & A & & & & & & & & & A & & & \\
\hline & & & & & & & & & & & A & C & & 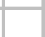 & & & & & \\
\hline & & & & & & & A & & & & & & & & & A & & C & \\
\hline & & A & & & & & & & & & C & & C & & & & & & \\
\hline & & & & C & & & & & & C & & & & & & & & & \\
\hline 15 & & & & & & & A & & & & A & C & & & & & & & A \\
\hline & & & & & & & & & C & & & & & & & & A & & C \\
\hline & & A & & & & & & & & & & & & & A & & & & \\
\hline & & & & & & & & & & & & & A & & & & & & \\
\hline & & & C & A & & & & & & C & & & & & & & & & \\
\hline 20 & & & & & & & C & A & & & & & C & A & C & & & A & \\
\hline
\end{tabular}

Figure III-4.43. Initial arrangement of anodic ('A'), cathodic (' $\mathrm{C}$ '), and neutral (blank) sites, Run B; all runs used the same initial settings, so this is representative of all initial conditions.

\begin{tabular}{|c|c|c|c|c|c|c|c|c|c|c|c|c|c|c|c|c|c|c|c|c|}
\hline RowsICols & 1 & & & & 5 & & & & & 10 & & & & & 15 & & & & & 20 \\
\hline 1 & & C & $A$ & & & & & & & & & & & & A & & & C & & \\
\hline & A & & & & & & & & & & & A & & & & & & & & \\
\hline & & C & & & & & & A & C & A & & & & A & C & C & C & & & A \\
\hline & A & C & & & A & & & C & & & & C & C & C & & A & & & & \\
\hline 5 & & C & A & & C & & & A & & & C & A & & A & & & & & & \\
\hline & A & & & & $C$ & A & & & & A & & & & & & & & & & \\
\hline & & & C & & $C$ & & & & & & & & A & & A & C & & A & & \\
\hline & & & C & A & & $A$ & C & A & & $A$ & & C & C & C & & & C & & & C \\
\hline & & & & & & & & & & & C & & $A$ & C & & & & & & C \\
\hline 10 & $A$ & & A & & & & & A & & & & & & & A & & A & & C & \\
\hline & & & C & & & & C & C & & A & & A & & & & & & & & C \\
\hline & & & & & & C & A & & & & C & & & & & & A & & C & \\
\hline & & & C & A & & & C & C & C & A & & & A & & & & & & & \\
\hline & & & C & C & & & A & & & C & & & & & & & A & & & A \\
\hline 15 & C & & & A & & & C & & A & C & A & & & A & & & & & & \\
\hline & C & C & & & C & C & & & & & & & & C & & & A & & & \\
\hline & $A$ & & C & C & A & & A & & $A$ & C & A & & $A$ & & & & & C & A & \\
\hline & & C & $A$ & C & & & & & & C & & & & & & C & A & & $C$ & C \\
\hline & & C & & & & A & & A & & & & C & A & & & & & & & \\
\hline 20 & & & & C & & & & & & C & $A$ & & C & & & & & & A & \\
\hline
\end{tabular}

Figure III-4.44. Final arrangement of anodic, cathodic, and neutral sites, Run B; Variables for this run were set so that all rules were in effect. 
Figure III-4.45 shows the final layout of Run C, where "cfCRand" was set to a high value. In this case, the probability was 0.08 . The probability of cathodes dying is so high that the anodes also die rapidly, because the current they are generating is not consumed. There are periods of time, seen in the current record of Figure III-4.44, in which a few anodic sites survive for a short time before their local cathodes die out. There are also periods where the current signal is dominated by the random birth and death of anodic sites and cathodic sites only, and not by any of the interaction mechanisms (i.e., sites are born and immediately die, generating the shortest possible fluctuations in current).

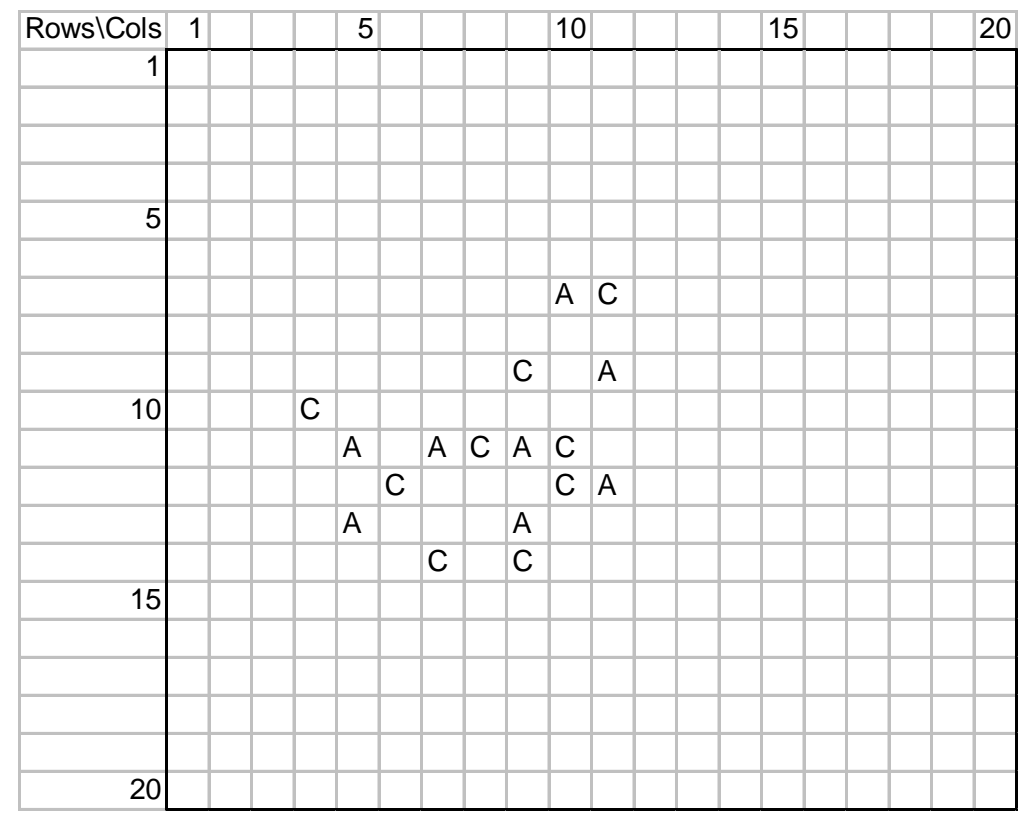

Figure III-4.45. Final arrangement of anodic, cathodic, and neutral sites, Run B; random cathodic death was set so high that the anodic sites die along with the cathodes, and thus much of the current data are dominated by the high-frequency birth and death of sites. 


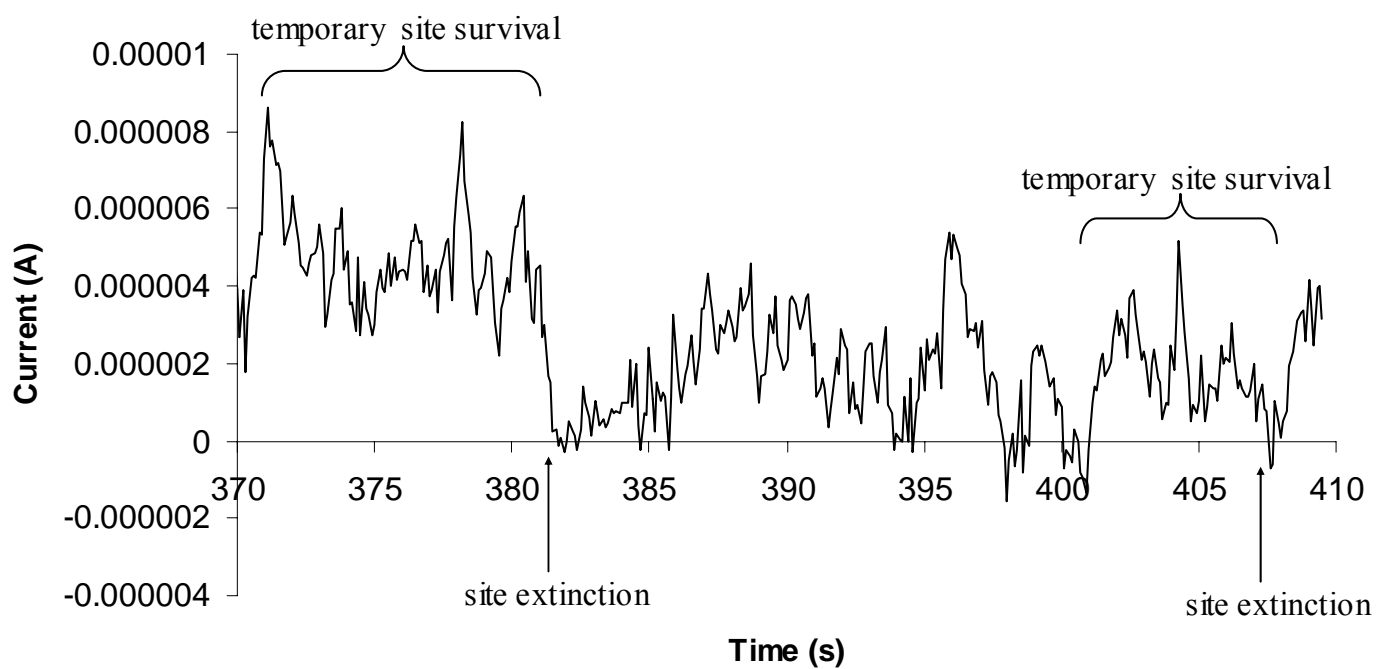

Figure III-4.36. Current record over 40 seconds of Run B; two periods of time are designated in which cathodes and anodes survived, dependent on each other, before the random death of cathodic sites caused the extinction of all sites.

Figure III-4.47 shows the results of WA on the current data from Run C. There is no low-frequency crystal increase, and there are mid-range crystal increases representing the temporary site survival periods. The two site survival periods shown in Figure III4.46 are approximately 10 seconds and 5 seconds in length, corresponding to crystals D6 and $\mathrm{D} 7$, respectively.

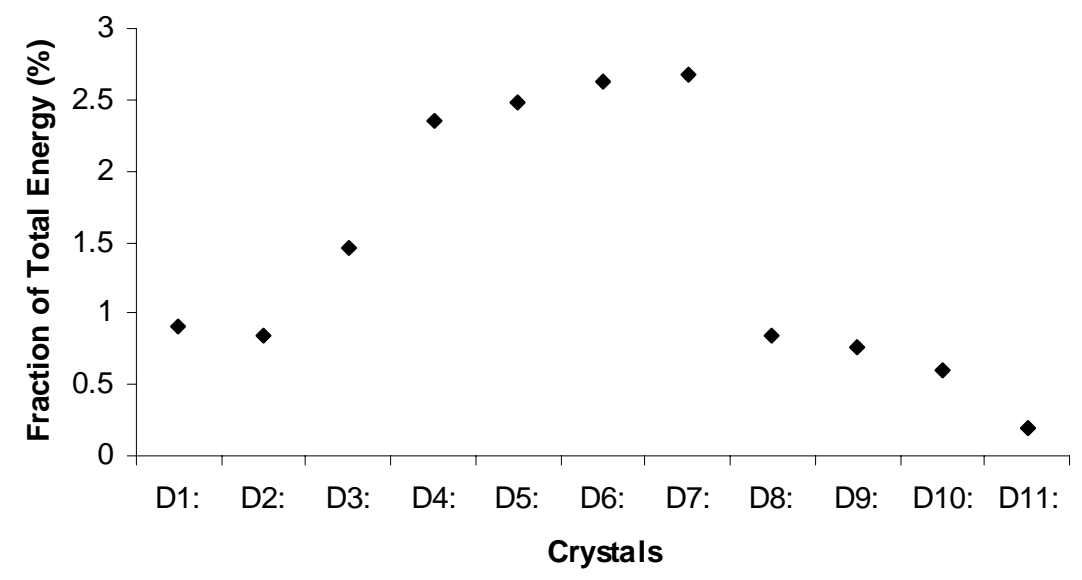


Figure III-4.47. WA of rule-based simulation Run C; mid-frequency crystal (D4-D7) contributions correspond to the short periods of anodic and cathodic site codependency and survival of Figure III-4.46.

Figures III-4.48 through III-4.50 show the results of Run D, where the variables governing the probability of cathodic sites being born near anodic sites were reduced. Reducing the probability too much causes the same kind of extinction as in Run C, shown in Figure III-4.48.

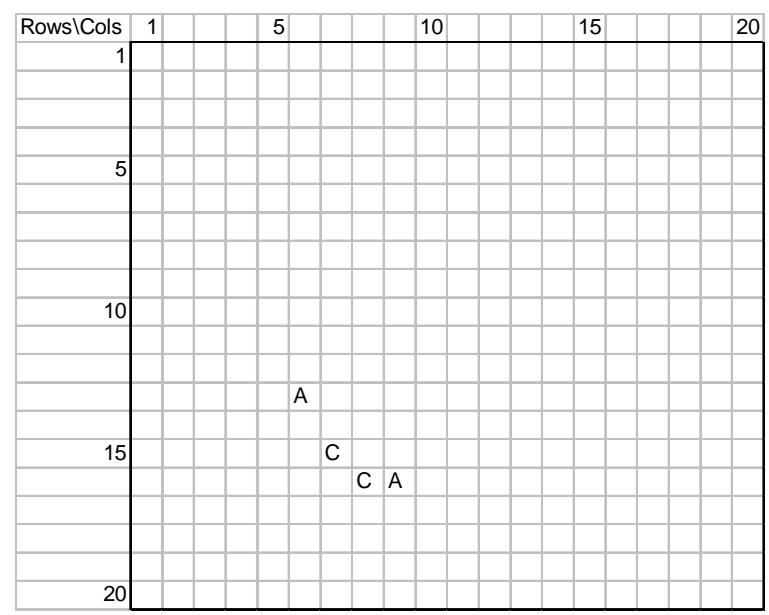

Figure III-4.48. Final layout of sites for simulation Run D; shows the same extinction of sites as seen in Run B (Figure III-4.45), this time due to decreasing the probability of new cathodic sites being born near existing anodic sites.

WA (Figure III-4.49) of the current data from Run D shows the same midfrequency trend as seen in Run C, as well as a low-frequency increase. This new increase is due to the large proportion of dependence on random current fluctuations, as seen in Figure III-4.50. 


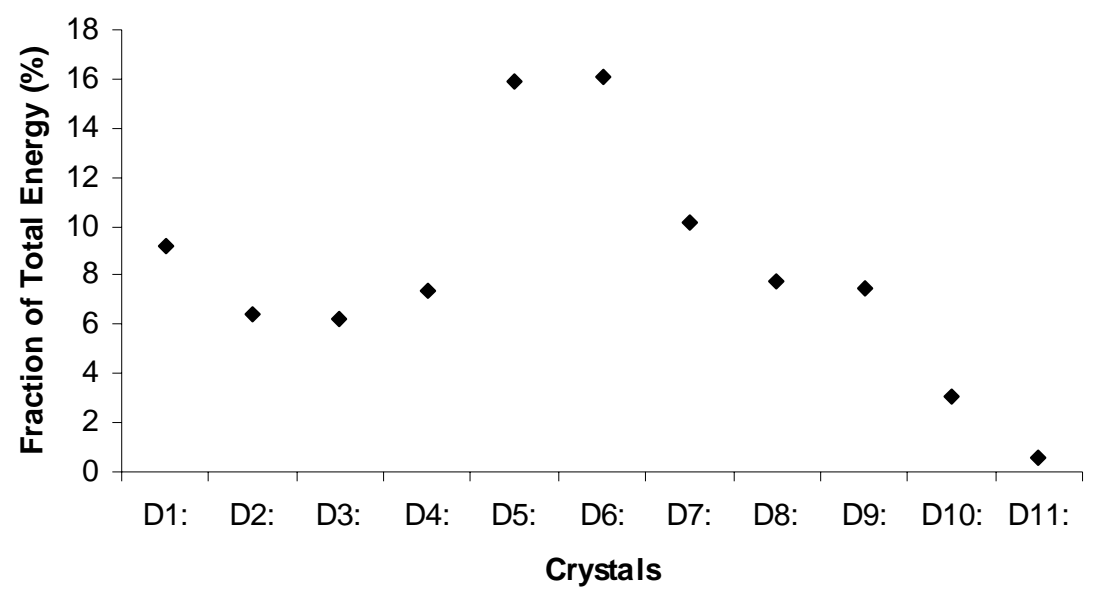

Figure III-4.49. WA of simulation Run D; shows a mid-frequency peak due to short-term anodic/cathodic site survival and a low-frequency increase because of long periods of current generation by random processes.

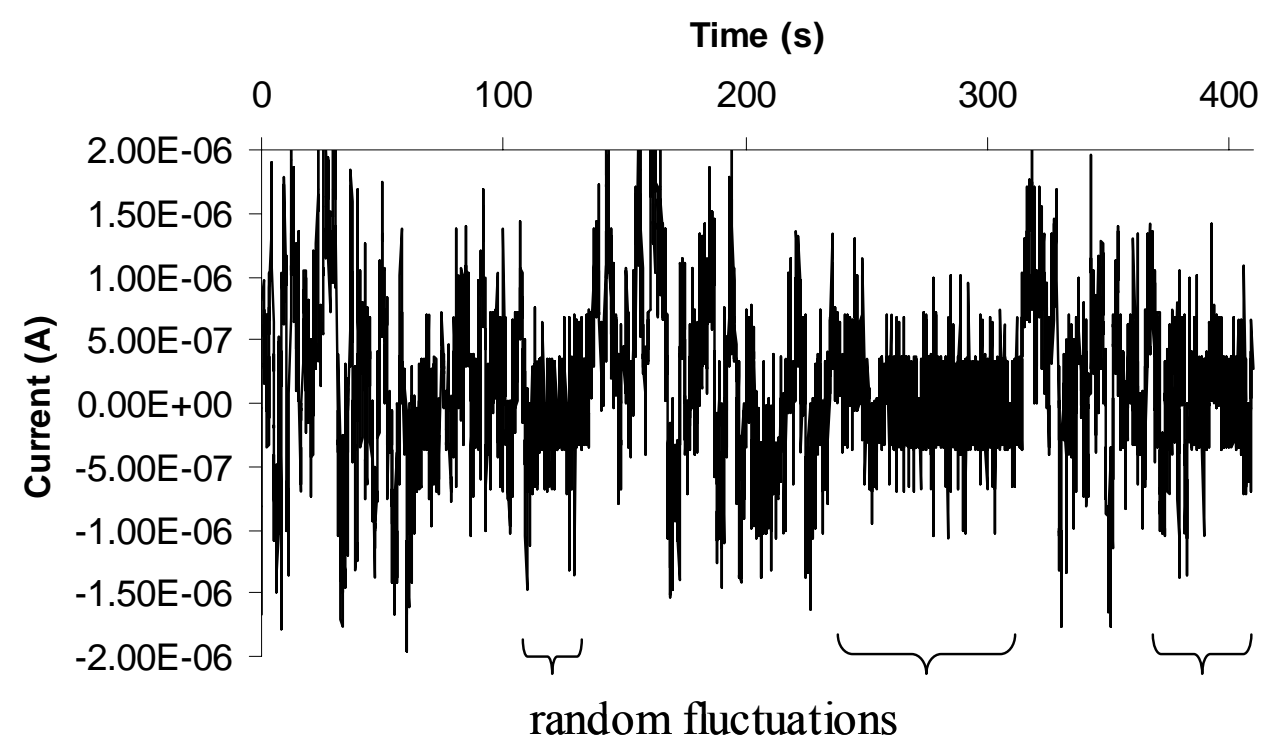

Figure III-4.50. Current record for simulation Run D; shows large periods of current fluctuations that are generated by random, high-frequency, processes. 
Figures III-4.51 and III-4.52 show the current data and WA for Run B, respectively. As stated above, all of the variables in this run were set to moderate values that allowed all rules to be active. The WA shows increases in two of the low-frequency crystals, D8 and D10. These increases indicated some low-frequency dependence, but the trend is not as strong as seen in some experimental runs. Crystals D7, D9, and D11 do not have large values: so values of the variables can be altered to generate better lowfrequency conditions.

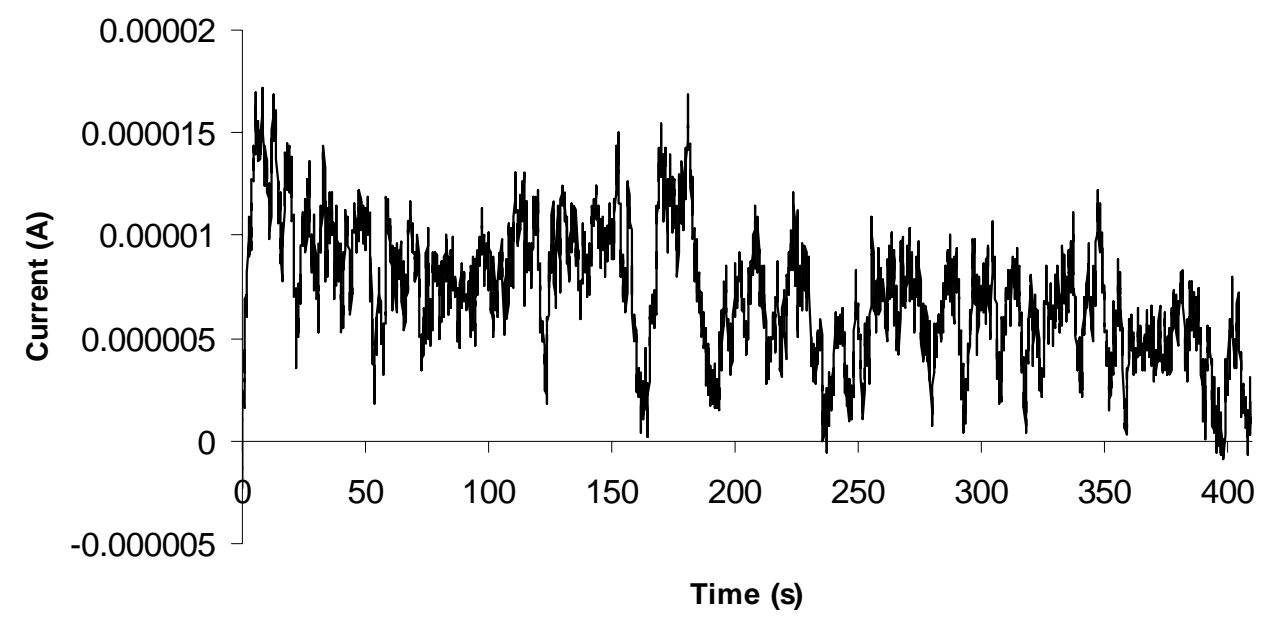

Figure III-4.51. Current record of simulation Run B. 


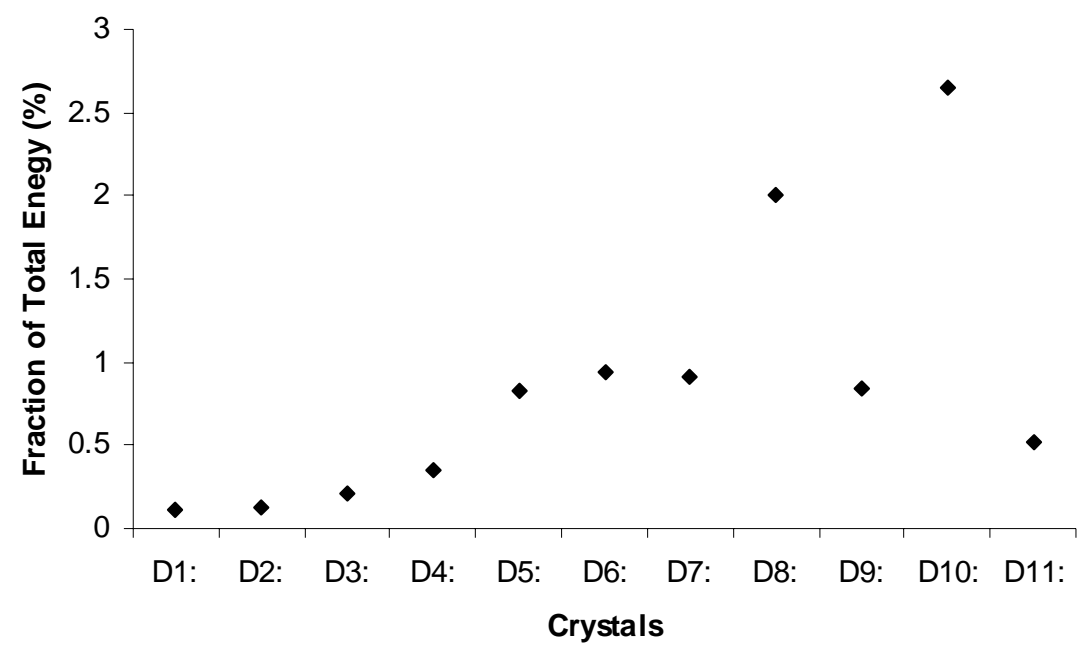

Figure III-4.52. WA of simulation Run B; increases in low-frequency crystals D8 and D10 show some low frequency dependence in the simulation, but the lower values for crystal D7, D9, and D11 indicate that there is room for improvement.

Figure III-4.53 shows the final arrangement of sites in Run E. For this run, "fCathFrac" was set to only 5 percent. Logically, this would mean that the anodic sites die rarely, and are limited in number mainly by their proximity to other anodic sites. This arrangement of sites is very similar to that of Run B (Figure III-4.54), with a slightly higher density of anodic sites.

\begin{tabular}{|c|c|c|c|c|c|c|c|c|c|c|c|c|c|c|c|c|c|c|c|c|}
\hline Rows ICols & 1 & & & & 5 & & & & & 10 & & & & & 15 & & & & & 20 \\
\hline 1 & & & C & $A$ & C & A & & & & & C & $A$ & & $A$ & & C & & & & A \\
\hline & & A & & C & & & C & A & & A & & & & & & A & & A & C & \\
\hline & & & C & A & & A & & C & & & & A & & A & & & C & & & C \\
\hline & & A & & & & & & A & C & C & C & & & & & A & C & A & & A \\
\hline 5 & & & C & C & A & C & & & & A & & A & & A & & & & & & C \\
\hline & C & A & & & & & C & & & C & & C & C & & & & C & A & & A \\
\hline & & & & & A & C & A & C & A & C & & A & & A & & A & & & & C \\
\hline & & & & & & & & & & & & C & C & & & C & & A & C & A \\
\hline & A & & & A & & A & & A & & A & C & A & & & A & C & & & & \\
\hline 10 & & C & C & C & & C & & & C & & & & & & C & & A & C & & \\
\hline & & A & & & & & & A & & A & C & & & & A & & & & A & C \\
\hline & & & & A & & A & & & & & & & A & C & C & & A & & & \\
\hline & & A & & C & C & & & & A & & & & & & & & & & & A \\
\hline & C & & & A & & A & & C & & & A & & A & & A & & A & & & C \\
\hline 15 & & A & C & & & & & A & & & & & & & & & & & C & \\
\hline & C & & & & & & & & & A & & & & A & C & & A & & & A \\
\hline & A & & C & A & & & A & C & & & & A & & & & & & & C & \\
\hline & & & & & & & & & A & C & & & & & A & & A & & A & \\
\hline & & C & A & & & A & & & & & & C & A & & & & & C & C & \\
\hline 20 & A & & & C & & & & A & C & A & C & & C & C & & A & & C & A & \\
\hline
\end{tabular}


Figure III-4.53. Final layout of sites for simulation Run E; similar to Run B, all rules were in effect. But, in this run, the cathodic current fraction was set lower so that the anodes, which represent pits, would survive longer, and there would be a more accurate simulation of pitting.

In this run, the number of anodes increases to a maximum, so that the number of cathodes increases to a maximum supportable by the number of anodes. The number of cathodes then fluctuates with time depending on the appropriate rules. Accordingly, the current increases to a maximum and then fluctuates about that value. The overall anodic or cathodic character of the simulation depends on the random probability of cathodic site death. The higher the probability of random cathodic death, the more anodic the overall character. Figure III-4.54 shows the WA of Run E where the probability of random cathodic death was set to 0.03 . It shows a trend like that of pitting corrosion conditions, and one that is stronger than the trend in Run B. The results from simulation Runs B and E show that with either the frequent or infrequent death of anodic sites, a good simulation of pitting corrosion can be accomplished.

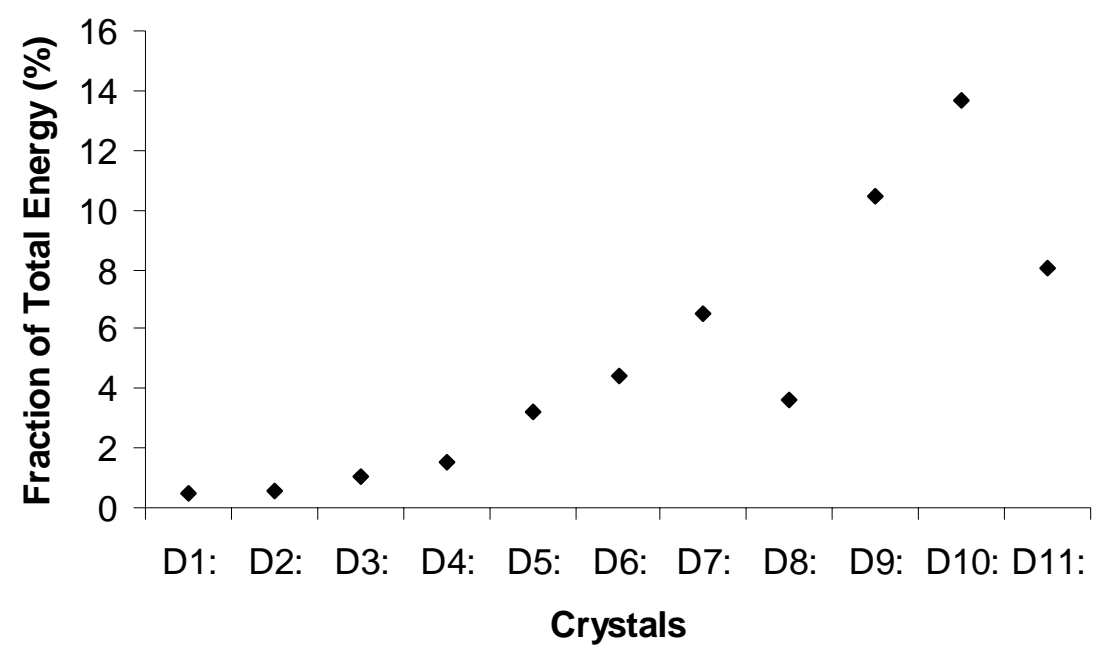

Figure III-4.54. WA of simulation Run E; increased contribution from the low-frequency crystals is similar to that seen in pitting corrosion conditions in experimental runs. 


\section{III-4.3g Summary}

Wavelet analysis was shown to identify various types of corrosion using the EN generated by corrosion processes. It was also able to identify the repassivation times for individual pitting events, as well as when signals contained contributions from more than one type of data, and when the type of corrosion changed. WA revealed the effects that a variety of chemicals present in waste storage containers have on the EN, and is an effective way to examine other chemicals in the future.

WA provided a means of testing and improving the simulation of corrosion processes on a material's surface. The simulation was able to accurately emulate the noise generated by general corrosion as well as pitting corrosion. 


\section{III-4.4 Conclusions and Future Work}

In this work, the examination of electrochemical noise data comprised three main approaches: one, a computer simulation of the anodic and cathodic activity relating to corrosion on a metal surface; two, experimental modeling of the electrochemical environment inside nuclear waste storage containers and collection of EN generated; and three, Wavelet analysis of the EN data from the first two parts.

The simulation of EN proved to be effective in replicating the EN data of both general and pitting corrosion. Using competition mechanisms for the anodic and cathodic sites on the surface, the long-term, low-frequency data generated by localized pitting corrosion was reproduced. Disabling one or more of the rules of the simulation eliminated the low-frequency character of the data, and eliminating all of the rules effectively reproduced general corrosion noise. The simulation accuracy benefited from comparison to experimental data, and conversely, it improved the EN analysis by providing theory for the underlying mechanisms.

The experimental electrochemical cell modeled the important factors in nuclear waste storage containers for this EN study; mainly increased temperature and the concentrations of corrosion-inducing or inhibiting chemicals. It also provided a platform for studying how the EN was affected by the competing chemicals.

The third and most important aspect of this study was Wavelet analysis. It proved to be an effective means of studying EN data generated by corrosion processes. Taking a deterministic approach, WA collects information about the underlying corrosion processes that has not been reliably obtained by previously established analysis methods. The primary advantage WA has over competing methods like FFT and MEM is that it is specifically adapted to the characteristics of EN. WA is able to work with the rapid, overlapping fluctuations in current data and with the changing mean. It also analyzes the data at a number of frequency resolutions and retains data in the temporal domain.

Both FFT and WA are frequency domain analysis methods. WA identifies the EN generated by different corrosion processes in the frequency domain, but FFT is simply not able to provide the detail and resolution that wavelets can. Wavelet analysis' division of information into a smooth data crystal and many detail data crystals allow it to more- 
accurately depict the high-frequency and/or low-amplitude noise fluctuations key to EN. These are the fluctuations that contain information on the underlying corrosion processes, but, in FFT, that information is mostly lost when analyzed at only one frequency resolution and depicted on a $\log$ scale. In WA, the increasing resolution of subsequent crystals provides information on a number of time scales, which is key to analyzing the different types of corrosion because of the natural frequencies of the corrosion mechanisms.

In this work, it was shown that WA identifies when general corrosion is the prevailing corrosion type because it has an inherently high frequency. Pitting corrosion is similarly identified by its lower frequency. WA also identifies when there is a mix of corrosion types by examining the contributions from both low- and high-frequency crystals to the total signal energy. Depictions of the wavelet coefficients in the time domain identify at what time a corrosion type becomes dominant or fades based on when the standard deviation of the wavelet coefficients increases or drops, respectively. It also accurately determines the average duration for many corrosion events, and it is able to resolve the repassivation times of single events.

WA also determined that nitrate ions are effective in inhibiting both general and localized corrosion, while nitrate ions are less effective at inhibiting localized corrosion and instead promote general corrosion. Phosphate ions promote general corrosion, and chloride ions induce pitting (in accordance with literature). 
Further work would comprise expanding the simulation to incorporate more of the rules governing site activity. One focus point would be to define rules to govern the formation and growth of single, large, stable pits whose current transients were seen in many experimental runs but not specifically simulated. WA would also be used to examine other corrosion types like SCC and crevice corrosion. Also, the ability of WA to identify types of corrosion would be developed into a quantitative measure defining at what fractional energy contributions at specific frequencies are no longer decisive in determining the dominant corrosion mechanisms and on what factors (current density, ratio of sites, etc.) these fractions are dependent.

1. Macdonald, D.D., "Passivity - the key to our metals-based civilization," Pure andApplied Chemistry, vol. 71, no. 6, (1999) p. 951-978.

2. Manahan, M.P., and Macdonald, D.D., "Determination of the Fate of the Current in the Stress Corrosion Cracking of Sensitized Type 304SS in High Temperature Aqueous Systems," Corrosion Science, vol. 37, no. 1 (1995) p. 189-208.

3. Latha, G., Rangarajan, S., and Narasimhan, S.V., "Electrochemical investigation of carbon steel corrosion and its inhibition in a dilute decontamination chemical," Corrosion Prevention \& Control, vol. 50, no. 1, (March 2003) p. 35-42.

4. King, R.B., Bhattacharyya, N.K., Wiemers, K.D. "Noble metal catalyzed hydrogen generation from formic acid in nitrite-containing simulated nuclear waste media" Environmental Science and Technology, Vol. 30, no. 4, 1996, p. 1292-1299.

5. Toghiani, R., Lindner, J., Phillips, V., and Selvaraj, D. "Saltcake Dissolution" Instrumentation development, measurement and performance, Evaluation of Environmental Technologies: Hanford Tank Waste Chemistry. available at: www.dial.msstate.edu/reports/40395R12.pdf

6. Campbell, S. G., Geeting, M. W., Kennell, C. W., Norato, M. A., Pierce, R. A., Walker D. D., and Wilmarth, W. R., "Demonstration of Caustic-Side Solvent Extraction with Savannah River Site High Level Waste," WSRC-TR-2001-00223, available at: http://www.osti.gov/bridge/

7. Crawford, C. Barnes, D., "Evaporation of Hanford Tank Sample AN-107 Mixed with Recycles (U)" available from www.osti.gov/bridge

8. Barnes, M., Sherwood, D., Barnes, C.D., and Edwards, T.B. "High Level Waste Lag Storage and Feed Blending" available from www.osti.gov/bridge 
9. Cheng, Y. F., Wilmott, M., and Luo, J. L. "The role of chloride ions in pitting of carbon steel studied by the statistical analysis of electrochemical noise" Applied Surface Science, Volume 152, Issues 3-4 , 2 November 1999, Pages 161-168.

10. Press, W.H., Teukolsky, S.A., Vetterling, W.T., and Flannery, B.P. Numerical Recipes in C++: The Art of Scientific Computing. Second Edition. New York City: Cambridge University Press, 2002. pp. 596-603

11. Dr. Mirna Urquidi-Macdonald, personal correspondence.

12. Zhang, Z., et al. "Electrochemical Noise Analysis of Pure Aluminum in Sodium Chloride Solution with Wavelet Transform Technique." Acta metallurgica Sinica vol. 15 , no. 3 (2002): 272-278.

13. Liu, X.F., et al. "Analysis of Electrochemical Noise with Wavelet Transform." Corrosion vol. 57, no. 10 (2001): 843-852.

14. Turnbull, A., Coleman, D., Griffiths, A.J., Francis, and P.E., Orkney, L. "Effectiveness of corrosion inhibitors in retarding the rate of propagation of localized corrosion," Corrosion, vol. 59, no. 3 (2003) p. 250-257.

15. Ballanger, G. and Rameau, J.J. "Inhibition of chloride pitting corrosion of zircaloy-4 alloy in highly radioactive water by radiolytic nitrate and hydrogen peroxide," Journal of Materials Science, vol. 35, no. 7 (2000) p. 1759-1771.

\section{III-5. Task: Combining the Deterministic and Statistical Approaches for Predicting Localized Corrosion Damage}

\section{III-5.1. Developing a Theoretical Basis for DFA}

\section{$\underline{\text { Task Status }}$}

Localized corrosion damage in an arbitrary system is completely defined if we know how many pits or other corrosion events $\left(\right.$ per $\mathrm{cm}^{2}$ ) have depths between $x_{1}$ and $x_{2}$ $\left(x_{1}<x_{2}\right)$ for a given observation time, $t$. Let us denote this number by $\Delta N_{k}\left(x_{1}, x_{2}, t\right)$. Here, index $\mathrm{k}$ denotes different types of corrosion event (e.g., pits or cracks). Instead of employing a function with three variables, $\Delta N_{k}$, it is more convenient to use a function of two variables - the integral damage function (IDF), $F_{k}(x, t)$. This function is defined as the number $\left(\right.$ per $\mathrm{cm}^{2}$ ) of corrosion events with depths larger than $\mathrm{x}$ for a given observation time, $t$. It is evident that 


$$
\Delta N_{k}\left(x_{1}, x_{2}, t\right)=F_{k}\left(x_{1}, t\right)-F_{k}\left(x_{2}, t\right)
$$

In turn, it is convenient to express the integral DF, $F_{k}(x, t)$, via the differential DF, $f_{k}(x, t)$, using the relation

$$
F_{k}(x, t)=\int_{x}^{\infty} f_{k}\left(x^{\prime}, t\right) d x^{\prime} \quad \text { or } \quad f_{k}(x, t)=-\frac{\partial F_{k}(x, t)}{\partial x}
$$

The differential DF for defects of type $k, f_{k}$, is defined from the condition that $f_{k}(x, t) d x$ is the number of defects $k$ (per $\mathrm{cm}^{2}$ ) having a depth between $x$ and $x+d x$ for a given observation time, $t$. It is evident that the set of functions $f_{k}$ for all types of defects yields complete information about damage in the system. The advantage of using the differential DF lies in the fact that it obeys a simple differential equation (see below) and accordingly can be calculated for any given set of conditions. Of course, all of the functions $\Delta N_{k}\left(x_{1}, x_{2}, t\right), F_{k}(x, t)$, and $f_{k}(x, t)$ may depend, in the general case, on position on the metal surface, but in this analysis we will assume that all sites belong to a given ethnic population.

The function $f_{k}$ has dimension of $\# /\left(\mathrm{cm}^{2} \mathrm{~cm}\right)=\# / \mathrm{cm}^{3}$ (analogous to the concentration of a particle). Accordingly, it is very convenient to regard each defect as a "particle" that moves in the $\mathrm{x}$ direction (perpendicular to the surface, with $\mathrm{x}=0$ being at the metal surface). The coordinate of this particle, $x$, coincides with the depth of penetration of the defect. Accordingly, $f_{k}$ can be regarded as the concentration of particles and hence must obey the law of mass conservation,

$$
\frac{\partial \mathrm{f}_{\mathrm{k}}}{\partial \mathrm{t}}+\frac{\partial \mathrm{j}_{\mathrm{k}}}{\partial \mathrm{x}}=\mathrm{R}_{\mathrm{k}}, \quad k=1,2, \ldots, K
$$

where $j_{k}$ and $R_{k}$ are the flux density and the bulk source (sink) of the "particles" $k$, respectively. Thus, the subscript " $k$ " enumerates the corrosion defect and " $K$ " is the total number of different corrosion defects in the system. By definition $R_{k}(x, t) d x d t$ yields the number of defects $k$ (per $\mathrm{cm}^{2}$ ) with depths between $x$ and $x+d x$ that arise (or disappear) 
during the period of time between $\mathrm{t}$ and $t+d t$, due to the transformation (repassivation, in the case of pits).

This new formulation of DFA affords considerable advantage over the previous treatment $[1,2,5]$ : we now possess a method of calculating the DF for a complicated system. To fulfill this task, we must solve the system of Equations (3) with the corresponding boundary and initial conditions.

$$
j_{k}=n_{k}(t) \text { at } x=0, t>0
$$

and

$$
f_{k}=f_{k 0}(x) \text { at } x>0, t=0
$$

where $f_{k 0}(x)$ is the initial distribution of defect $k$ [usually we can assume that $f_{k 0}(x)=0$ ), i.e., no damage exists at zero time] and $n_{k}(t)$ is the nucleation rate of the same defect [i.e, $n_{k}(t) d t$ is the number of stable defects $\left(\right.$ per $\left.\mathrm{cm}^{2}\right)$ that nucleate in the induction time interval between $\mathrm{t}$ and $t+d t]$.

Thus, because the defect propagation flux, $j_{k}$, must be non- negative (the depth of a corrosion event can only increase with time), the following, simplest numerical upwind finite difference scheme can be used for numerically solving Equations (3) - (5).

$$
f_{k, m}^{n+1}=f_{k, m}^{n}-\frac{\Delta t}{\Delta x}\left(j_{k, m}^{n}-j_{k, m-1}^{n}\right)+R_{k, m}^{n} \Delta t
$$

Here, we use the straightforward approach of choosing equally spaced points along both the t- and $\mathrm{x}$-axes: $x_{j}=x_{0}+m \Delta x, \quad m=0,1, \ldots, J$ and $t_{j}=t_{0}+n \Delta x, \quad n=0,1, \ldots, N$, and we denote $f_{k, m}^{n}=f_{k}\left(t_{n}, x_{m}\right)$. The values $f_{k, 0}^{n}$ and $f_{k, m}^{0}$ are calculated from the boundary and initial Conditions (4) and (5). Of course, it is assumed that we know (i.e. can calculate) fluxes, $j_{k}$, and sources, $R_{k}$, as functions of the spatial coordinates and time, and, in the general case, as functions of the unknown values of $f_{k}$. 
In the simplest cases, it is even possible to obtain analytical solutions for the damage functions. As an example, let us consider the case of pitting corrosion under constant external conditions. In this case, we have two kinds of defect $(K=2)$ : active pits with the damage function, $f_{a}$, and passivated pits (i.e., those that have "died" through delayed repassivation) with the damage function, $f_{p}$. Here, and elsewhere in this paper, subscripts " $a$ " $(k=1)$ and " $p$ " $(k=2)$ denote active and passive pits, respectively.

Let us assume that the rate of pit propagation, $V$, depends only on the depth of the pit, $x$. Accordingly, for the flux of active pits, $j_{a}$, we have:

$$
j_{a}(x, t)=f_{a}(x, t) V(x)
$$

Generalization of this expression will be done later. However, it worth noting that the expression for the flux density cannot contain "diffusion terms", because pits can only increase their depth and the flux does not depend on the gradient in the pit population and hence on the differential damage function. By definition, the flux of passivated pits, $\mathrm{j}_{\mathrm{p}}$, is zero (i.e., these pits are "dead"). It is also evident that functions $R_{a}$ and $R_{p}$ must obey the relation $R_{p}=-R_{a}$, since the rate of appearance of passived pits must equal the rate of disappearance of active pits, due to passivation, at any given moment in time. If, in addition, we assume that the pit repassivation process obeys a first order decay law (the rate of passivation is proportional to the number of active pits), the function $R_{a}$ has the form

$$
R_{a}(x, t)=-\gamma f_{a}(x, t)
$$

where $\gamma$ is the delayed repassivation ("death") constant (i.e., the rate constant for repassivation of stable pits). The negative sign indicates that the number of active pits decreases due to repassivation. In the general case, $\gamma$ depends on the external conditions and, accordingly, on the depth of the pit, if significant potential and concentration drops exist within the cavity. In addition, $\gamma$ may also depend on time when the external conditions vary. However, in this example, we assume that $\gamma$ is a constant and hence that the probability of repassivation does not depend on pit depth. (This is clearly a gross 
over-simplification, since the probability of delayed repassivation is expected to increase with pit age, but this does not change the logic of the argument). Accordingly, the system of equations for calculating the DFs has the form

$$
\frac{\partial f_{a}}{\partial t}+\frac{\partial\left(V f_{a}\right)}{\partial x}=-\gamma f_{a}
$$

and

$$
\frac{\partial f_{p}}{\partial t}=\gamma f_{a}
$$

The boundary and initial conditions are then given as

$$
V f_{a}=n(t) \text { at } x=0, t>0 \quad \text { and } \quad f_{a}=f_{p}=0 \quad \text { at } t=0
$$

The difference between Equations (9) and (10) is as follows: Equation (9) shows that the number of active pits can change due to the nucleation of new pits and the repassivation of existing (active) pits. In contrast, the number of dead pits can change only due to the repassivation of active pits (Equation 10).

An analytical solution to the boundary value problem formulated above yields

$$
f_{a}=\frac{\exp [-\gamma \theta(x)] n[t-\theta(x)]}{V(x)}
$$

and

$$
f_{p}=\frac{\gamma \exp [-\gamma \theta(x)] N[t-\theta(x)]}{V(x)}
$$

where

$$
\theta_{p i t}(x)=\int_{0}^{x} \frac{d x^{\prime}}{V\left(x^{\prime}\right)}
$$

is the age of a pit with depth $x$ and 


$$
N(t)=\int_{0}^{t} n\left(t^{\prime}\right) d t^{\prime}
$$

is the number of stable pits $\left(\right.$ per $\mathrm{cm}^{2}$ ) that nucleate in the time interval between 0 and $t$.

It is important to note that, experimentally, only the sum of the damage functions for active and passive pits, $f=f_{a}+f_{b}$, is determined and, in many instances, the integral damage function, $\mathrm{F}=\mathrm{F}_{\mathrm{a}}+\mathrm{F}_{\mathrm{p}}$, only is measured. Accordingly, it is important, for practical reasons, to obtain the equation for the integral damage function. Note that the integral damage function, $\mathrm{F}$, corresponds to the number of remaining pits (active and passive) on a surface, as the surface is removed layer-by-layer in pre-established increments. From Equations (2), (12), and (13) we therefore have

$$
F(x, t)=\exp [-\gamma \theta(x)] N[t-\theta(x)]
$$

According to the theory developed above, calculation of the damage functions requires the determination of three independent functions for each kind of corrosion defect, $k$ : The rate of defect nucleation, $n_{k}$, the flux density (growth rate) of the defect, $j_{k}$, and the rate of transition of one kind of defect to another, $R_{k}$, (for example, the transition of an active pit into a passive pit or the transition of a pit into a crack). Below, we will discuss briefly the feasibility of calculating each of these three functions from first principles.

In many practical cases, it is possible to assume that all pits on a given surface nucleate during an initial period of time that is much less than the observation time, $t$, or the service life of the system, $\mathrm{t}_{\mathrm{s}}$ (instantaneous nucleation). For example, for the case of the pitting corrosion of aluminum in tap water, practically all of the pits were found to nucleate within the first two weeks [9]. Under these conditions, the total number of nucleated stable pits, as a function of time, can be simply represented as

$$
N(t)=N_{0} U_{+}(t)
$$


where $N_{0}$ is the maximum number of the stable pits $\left(\right.$ per $\left.\mathrm{cm}^{2}\right)$ that can exist on the metal surface and $U_{+}(t)$ is the asymmetrical unit function $\left(U_{+}=0\right.$ at $t \leq 0$ and $U_{+}=1$ at $\left.t>0\right)$.

If pit nucleation cannot be regarded as being "instantaneous", the simplest assumption concerning the pit nucleation rate, $n(t)=d N / d t$, is that $n(t)$ is proportional to the number of available sites, $N_{0}-N(t)$ [10], which yields.

$$
N(t)=N_{0}\left[1-\exp \left(-t / t_{0}\right)\right]
$$

where $t_{0}$ is some characteristic time that depends on the corrosion potential, temperature, and electrolyte composition.

A more general and sophisticated calculation of the pit nucleation rate can be made in the following way. Although an extensive database does not exist to support this position, it is postulated that the rate of nucleation of stable pits, $n=d N / d t$, is related to the rate of nucleation of metastable pits, $n_{M P}=(d N / d t)_{M P}$, by

$$
n(t)=\frac{d N}{d t}=\zeta\left(\frac{d N}{d t}\right)_{M P}
$$

where parameter $\zeta$ is termed the survival probability [10-12]. This parameter can be measured experimentally. Thus, for example, for Type 304L stainless steel in $\mathrm{NaCl}$ solution, the experimentally measured survival probability has a value of the order of $10^{-2}$ [12].

For calculating the rate of nucleation of metastable pits, the Point Defect Model (PDM) can be used [13-17]. On the basis of this model, it can be shown that total number of nucleated pits can be described by the following expression [5]

$$
N(t)=N_{0} \operatorname{erfc}\left(\frac{a}{t}+b\right) / \operatorname{erfc}(b)
$$

Parameters $a$ and $b$ specifically depend on temperature, $\mathrm{pH}$, activity of halide ion, and the metal potential, but do not depend on the induction time. These dependencies can be 
found in the original work [5, 13-17]. To the best of our knowledge, the PDM is the only model that establishes the connection between pit nucleation rate and the external conditions. For example, in the empirical Equation (18), the connection between the parameter, $t_{0}$, and the external conditions $(\mathrm{pH}$, potential, etc.) is not specified. It is interesting to note that, if the observation time, $t$, satisfies the condition $t>>a\left(b+\sqrt{b^{2}+4}\right) / 2$, practically all pits nucleate during a very short period of time at the beginning of experiment, i.e. the PDM predicts the limiting case of instantaneous nucleation [5].

However, it is also important to note that the original PDM describes pit nucleation for the case when the external conditions do not depend on time. This restriction can be a serious impediment in predicting the pit nucleation rate under practical conditions, for example, when seasonal changes in temperature and wetness are observed for a system that is subject to atmospheric corrosion. However, the PDM is easily generalized for the case of variable external conditions, as described in the Appendix.

The quantitative description of pit (or crack) growth can be regarded as one of the key problems in predicting corrosion damage in many practical systems. This follows from the fact that the calculated corrosion damage that is based only on this (growth) stage can be compared with experiment, in many limiting cases. For example when all pits nucleate "instantaneously", or when the induction time for pit nucleation is much smaller than the observation time, it is possible to ignore the initial stage of pit nucleation when estimating the damage. In addition, if the probability of survival of a corrosion defect is sufficiently high, we must take into account the possibility that a stable corrosion defect (pit or crack) nucleates immediately after the start of operation and propagates without repassivation. In any case, calculations based only on the growth stage yield the most conservative estimate of the service life, $t_{s, \min }$, of the system. We can be sure that, if calculation of the service life is based on growth alone, the real service life, $t_{s,}$ will at least be not less than $t_{s, m i n}$.

It is well known from both experiment [18] and theory [19] that the dependence of the characteristic dimension of a corrosion cavity (for example, cavity depth, $L$ ) on time, $t$, can be expressed by a simple equation of the following form 


$$
L=k t^{m}
$$

where $k$ and $m$ are empirical constants, and, usually, $m \leq 1$. However, this dependence of $L$ on $t$ cannot be used directly in mathematical calculations for small times, because of the non-physical limit

$$
V=\frac{d L}{d t}=k m t^{m-1} \rightarrow \infty, \quad \text { at } t \rightarrow 0 \text { and } m<1
$$

This is why, instead of Equation (21), we use the following interpolation equation for pit propagation rate

$$
V=\frac{d L}{d t}=V_{0}\left(1+t / t_{0}\right)^{n}
$$

where $\mathrm{n}=\mathrm{m}-1$ and $\mathrm{t}_{0}$ are constants, and $V_{0}$ is the initial, finite rate of pit propagation. Equation (23) yields $V=V_{0}$ at $t / t_{0}<<0$ and $V=V\left(t / t_{0}\right)^{n}$ at $t>>t_{0}$.

It is important to note that, in many cases, the period of time over which the approximation

$$
V(x) \approx V_{0}=\text { constant }
$$

is valid can be comparable with the observation time (or even with the service life of the system). The reason is that corrosion is, generally speaking, a slow process and under real, practical conditions, values of the critical pit depth of the system, $x_{c r}$, (e.g., wall thickness of a pipe) and typical service life, $t_{s}$, impose significant restrictions on the values of the initial and average corrosion current densities and, thus, on the potential and concentration drops that might be observed in a corrosion cavity [20]. Thus, as follows from Faraday's law, if $x_{c r}$ does not exceed the order of $1-10 \mathrm{~mm}$, and if the order of $t_{s}$ is not less than 1 year, the initial corrosion current densities in real pits cannot exceed 
values of $10^{-4}-10^{-3} \mathrm{~A} / \mathrm{cm}^{2}$, with the understanding that the polarization curve (corrosion current density vs. potential) does not change as the pit propagates [20]. Such low current densities can arise only from relatively low values of the potential and concentration drops in open pits. In particular, it is shown that if localized corrosion takes place in an electrolyte having a conductivity of the order of that of sea water, it is possible to neglect the potential drop in an open corrosion cavity and hence it is possible to regard the rate of pit propagation as being constant under constant environmental conditions. The same conclusion holds for the pitting of 316L stainless steel for practically any composition of the external electrolyte. This issue is discussed in greater depth in Ref. 20.

As noted above, we assume that the repassivation process obeys a first-order decay law [see Equation (8)]. The repassivation constant, $\gamma$, is, in general, a function of the external conditions, including the corrosion potential, temperature, and electrolyte composition. Generally speaking, $\gamma$ is also a function of the depth of the pit, $x$, because the local potential in the solution at the cavity surface depends on $x$, i.e. $\gamma$ might be a function of both the spatial coordinates and time. Of course, if the potential and concentration drops inside the corrosion cavity are insignificant during pit propagation, it is possible to neglect changes in parameter $\gamma$ (see above). In the current investigation, we assume, for simplicity, and hence to facilitate the development of analytical expressions for the damage functions $f_{a}$ and $f_{p}$, that $\gamma$ is a constant [1]. Of course, the value of this constant still depends on the external conditions, such as potential, $\mathrm{pH}$, and concentration of aggressive species in the bulk electrolyte. However, we can imagine the situation when the potential and concentration drops inside the pit are significant and timedependent (for example, in extremely dilute solutions or in closed pits); in which case the probability of repassivation will be a function of pit depth and hence pit age. It also can be that active pits cannot exist if the potential, E, at the pit internal surface is less then some critical value, $\mathrm{E}_{\mathrm{cr}}$. Accordingly, if the value of $\mathrm{E}_{\mathrm{cr}}$ is reached at some pit depth, $\mathrm{x}_{\mathrm{cr}}$, active pits passivate and cannot penetrate further into the metal. Accordingly, Equation (16) cannot be used.

Issues and Concerns: $\quad$ None 


\section{III-5.2 Task: Generalization of PDM to Describe Passivity Breakdown (where the external conditions vary with time)}

\section{$\underline{\text { Task Status }}$}

In the original version of PDM [13-17], the pit nucleation rate was obtained from the criterion for pit initiation:

$$
\left(J_{c a}-J_{m}\right) \times(t-\tau) \geq \xi
$$

where $J_{c a}$ is the cation vacancy flux in the barrier layer of the passive film at the metal/barrier layer interface, $J_{m}$ is the rate of annihilation of the cation or metal vacancies at the same location, $t$ is the observation time, $\tau$ is the time of dissolution of the pit cap until rupture, and $\xi$ is the critical "areal" concentration $\left(\# / \mathrm{cm}^{2}\right)$ of condensed vacancies that are required for the separation of the barrier layer from the substrate metal. This latter parameter can be obtained from the potential sweep rate dependence of the apparent breakdown potential and can also be calculated from structural arguments [27, 28]. However, Criterion (A-1) is valid only if all external parameters (temperature, electrolyte composition, $\mathrm{pH}$, corrosion potential, etc.) do not depend on time, and, accordingly, $J_{c a}$, $J_{m}$, and $\xi$ are constants. In the general case, when the external conditions are timedependent, instead of Criterion (A-1) we must use the following criterion:

$$
\int_{\tau}^{t}\left[\left(J_{c a}\left(t^{\prime}\right)-J_{m}\left(t^{\prime}\right)\right] d t^{\prime} \geq \xi(t)\right.
$$

Note that $\xi$ corresponds to the number of cation vacancies per unit area condensed on the cation sublattice on the film side of the metal/barrier layer interface or the number of metal vacancies condensed on the metal lattice on the metal side of the interface, depending upon whether vacancy condensation occurs on the cation sub-lattice of the film or on the metal lattice, respectively. In any event, the value of $\xi$ is of the order of 
(2-3) $\times 10^{15} \mathrm{~cm}^{-2}$, depending upon the crystallographic faces involved [27, 28]. The principal time-dependence of $\xi$ most likely arises from expansion/contraction of the lattice as the temperature changes, in which case $\xi=\xi_{0} \exp \left[-2 \hat{\alpha}\left(T-T_{0}\right)\right]$, where $\hat{\alpha}$ is the coefficient of linear thermal expansion, $T$ is the Kelvin temperature, $T_{0}$ is the reference temperature, and $\xi_{0}$ is the critical vacancy concentration at $T_{0}$. For example, we evaluate $\exp \left[-2 \hat{\alpha}\left(T-T_{0}\right)\right]$ for nickel (a simple, ccp metal with $\hat{\alpha}=1.3 \times 10^{-5} \mathrm{~K}^{-1}$ [29]) to be 0.9981 for $T=373.15 \mathrm{~K}\left(100{ }^{0} \mathrm{C}\right)$ and $T_{0}=298.15 \mathrm{~K}\left(25{ }^{\circ} \mathrm{C}\right)$, assuming that vacancy condensation occurs on the metal lattice, from which we conclude that $\xi$ can be considered to be quasi-constant. However, this may not be the case for passivity breakdown on a liquid metal, such as gallium [30], because of the much larger coefficient of expansion.

In previous work [27, 28], $J_{m}$ has been estimated from $J_{m} \leq i_{\text {passive }}^{c} / n F$, where $i_{\text {passive }}^{c}$ is the steady-state passive current density at the point of film breakdown, $n$ is the number of electrons involved in the annihilation reaction, and $F$ is Faraday's constant. The inequality arises, because not all of the current may be carried by cation vacancies; indeed, in n-type passive films, such as those that form on iron and aluminum, where the principal point defects are either oxygen vacancies or metal interstitials, or both, the current density due to the flux of cation vacancies might be only a small fraction of the total current density. If the transport number of the cation vacancy $\left(t_{c v}\right)$ in the barrier layer is known by independent experiment, then the value of $J_{m}$ can be established precisely as $J_{m}=t_{c v} i_{\text {passive }}^{c} / n F$, where $i_{\text {passive }}^{c}$ is the passive current density at the point of breakdown in the absence of a redox couple in the solution. In any event, methods have been developed for determining the rate of annihilation cation vacancies at the metal/barrier layer interface, primarily by analyzing electrochemical impedance data [31], so that $J_{m}$ may be determined by independent experiment for any given set of external conditions. This leaves only $J_{c a}$ in Equation (A-2) to be determined by independent experiment, in order to affect a completely deterministic prediction of metastable pit nucleation rate. The rate constant for cation vacancy generation at the barrier layer/solution interface is readily determined using EIS and, for nickel, is found to be of 
the form $k=k_{0}+A\left[\mathrm{Cl}^{-}\right]^{p}$ [17], where $k_{0}$ is the rate constant for chloride free solutions and $A$ and $p$ are constants. For nickel in $\mathrm{NaCl}$ solutions [31], we have recently determined that $p=1$; that is, cation vacancy generation at the barrier layer/solution interface for passive nickel is first order in chloride concentration, a finding that is consistent with the Point Defect Model.

On any real surface, a large number of potential breakdown sites exist corresponding to a distribution in the properties of the "weak spots". Thus, examination of data reported by Shibata [32] and Fratesi [33], among others, suggests that the breakdown voltage is nearly normally distributed. The PDM assumes that the breakdown sites with respect to the distribution of diffusivity of cation vacancies is described approximately by a normal distribution function [17], i.e.

$$
\frac{d N(D)}{d D}=-A \exp \left[-\frac{(D-\bar{D})^{2}}{2 \sigma_{D}^{2}}\right]
$$

Here, $N(D)$ is the number of breakdown sites (per $\mathrm{cm}^{2}$ ) that have diffusivities larger than $D$, and $\bar{D}$ and $\sigma_{D}$ are the average value and the standard deviation, respectively, of the diffusivity for the population of the breakdown sites. The negative sign in Equation (A3 ) means that $N(D)$ decreases with increasing $D$. Parameter $A$ does not depend on $D$, so that normalization of the diffusivity distribution using the condition $N(0)=N_{t o t}$, where $N_{\text {tot }}$ is the total number of breakdown sites $\left(\right.$ per $\mathrm{cm}^{2}$ ), yields

$$
N(D)=N_{t o t} \operatorname{erfc}\left(\frac{D-\bar{D}}{\sqrt{2} \sigma_{D}}\right) / \operatorname{erfc}\left(-\frac{\bar{D}}{\sqrt{2} \sigma_{D}}\right)
$$

The cation vacancy flux density, $J_{c a}$, is proportional to the diffusion coefficient of vacancies, $D$, i.e.

$$
J_{c a}=D B,
$$


where the function $B$ depends on the on the external conditions (applied voltage, $V_{a p p}$, temperature, $T$, chloride activity, $a_{x}$, etc.). Thus, for the case of passivity breakdown in chloride solutions, the PDM yields:

$$
B=\hat{a} u^{-\chi / 2} \exp \left(\frac{\chi F \alpha V_{a p p}}{2 R T}\right) a_{x}^{\chi / 2}
$$

where $\alpha$ is the polarizability of the film/solution interface (i.e., dependence of the potential drop across the barrier layer/solution interface on the applied potential), $\chi$ is the cation oxidation state in the barrier layer, $R$ is the gas constant, and $F$ is Faraday's constant. In turn, parameters $\hat{a}$ and $u$ are defined as [13]

$$
\hat{a}=\chi(F \varepsilon / R T)\left[N_{A} / \Omega\right]^{1+\chi / 2} \exp \left(-\Delta G_{S}^{0} / R T\right)
$$

and

$$
u=\frac{N_{A}}{\Omega} \exp \left(\frac{\Delta G_{A}^{0}-\beta F p H-F \phi_{f / s}^{0}}{R T}\right)
$$

where $\varepsilon$ is the field strength in the film, $\Omega$ is the mole volume per cation, $\Delta G_{S}^{0}$ is the change of Gibbs energy for the Schottky-pair reaction $\left(\right.$ Null $\rightarrow V_{M}^{\chi^{\prime}}+(x / 2) V_{o}^{* \bullet}$, assuming that this is the cation vacancy generation process - see Ref. 20), $\beta$ is the dependence of the potential drop across the film/solution interface on $\mathrm{pH}, \phi_{\mathrm{f} / \mathrm{s}}^{0}$ is a constant (potential drop at the film/solution interface for $V_{a p p}=0$, and $p H=0$ ), and $\Delta G_{A}^{0}$ is the standard Gibbs energy change for the chloride absorption reaction $V_{O}^{\bullet \bullet}+\mathrm{Cl}_{a q}^{-} \rightleftharpoons$ $\mathrm{Cl}_{O}^{\bullet}\left(V_{O}^{\bullet \bullet}\right.$ is an oxygen vacancy in the film and $\mathrm{Cl}_{O}^{\bullet}$ is a chloride anion occupying a surface oxygen lattice site). A detailed discussion of these parameters is available in the original papers [13-17]. The assumption of a normal distribution of the breakdown sites 
with respect to $D$ results in a near-normal distribution in the breakdown voltage and a left-acute distribution in the induction time, in agreement with experiment [14-17].

Let us denote by $D_{0}$ the diffusivity of a cation vacancy at temperature $T_{0}$. We can calculate $D$ at any other temperature by using the relation

$$
D=D_{0} \omega
$$

where

$$
\omega=\exp \left[-\frac{E_{D}}{R}\left(\frac{1}{T}-\frac{1}{T_{0}}\right)\right]
$$

and $E_{D}$ is the energy of activation for diffusion.

Substituting Equations (A-5) and (A-9) into Equation (A-2) yields the following criterion for meta-stable pit nucleation as

$$
D_{0} \geq D_{c r}(t)=\frac{\xi+\int_{\tau}^{t} J_{m}\left(t^{\prime}\right) d t^{\prime}}{\int_{\tau}^{t} \omega B\left(t^{\prime}\right) d t^{\prime}}
$$

This criterion has to be understood in the following way. Let us assume that, at $t=0$ (at the beginning of corrosive attack), a potential breakdown site is characterized by a cation vacancy diffusivity of $D_{0}$. Criterion (A-11) states that the nucleation of metastable pits occurs within the observation time, $t$, on those and only on those sites that have $D_{0} \geq$ $D_{c r}(t)$.

Substitution of Equation (A-9) into Equation (A-4) yields an expression for the number of metastable pits (per $\mathrm{cm}^{2}$ ) that nucleate during the period of time $(0, t)$.

$$
N(t)_{M P}=N_{t o t} \operatorname{erfc}\left(\frac{D_{c r}(t)-\bar{D}_{0}}{\sqrt{2} \sigma_{D_{0}}}\right) / \operatorname{erfc}\left(-\frac{\bar{D}_{0}}{\sqrt{2} \sigma_{D_{0}}}\right)
$$


Subscript " 0 " in Equation (A-12) means that the values are calculated at temperature $T_{0}$, i.e. at $t=0$. Under constant external conditions, Equation (31) reduces to the previously developed expression [5]

$$
N(t)_{M P}=N_{t o t} \operatorname{erfc}\left(\frac{a}{t-\tau}+b\right) / \operatorname{erfc}(b)
$$

where $a=\xi /\left(B \sqrt{ } 2 \sigma_{\mathrm{D}_{0}}\right)$ and $b=\left(J_{m} / B-\bar{D}\right) /\left(\sqrt{2} \sigma_{D_{0}}\right)$.

By differentiating Equation (A-13), we obtain an expression for calculating the rate of nucleation of metastable pits:

$$
n_{M P}=\frac{d N_{M P}(t)}{d t}=\frac{\sqrt{2}}{\operatorname{erfc}\left(-\bar{D}_{0} / \sqrt{2} \sigma_{D_{0}}\right) \sqrt{\pi} \sigma_{D_{0}}} \exp \left[-\frac{\left(D_{c r}(t)-\bar{D}_{0}\right)^{2}}{2 \sigma_{D_{0}}^{2}}\right] \frac{d D_{c r}(t)}{d t}
$$

where, in accordance with Equation (A-11),

$$
\frac{d D_{c r}(t)}{d t}=\frac{d \xi / d t+J_{m}(t)}{\int_{\tau}^{t} \omega B\left(t^{\prime}\right) d t^{\prime}}-\frac{\omega B(t)\left(\xi(t)+\int_{\tau}^{t} J_{m}\left(t^{\prime}\right) d t^{\prime}\right)}{\left[\int_{\tau}^{t} \omega B\left(t^{\prime}\right) d t^{\prime}\right]^{2}}
$$

For the case when the external conditions do not depend on time, we have a more simple expression

$$
n_{M P}=\frac{N_{t o t} 2 a}{\operatorname{erfc}(b) \sqrt{\pi}} \frac{\exp \left[-\left(\frac{a}{t-\tau}+b\right)^{2}\right]}{(t-\tau)^{2}}
$$

which we previously derived [5]. 
It is important to note that all expressions for $N_{M P}$ or $n_{M P}$ are valid only for $t>\tau$. If $t \leq \tau$, we simply have $N_{M P}=0$ and $n_{M P}=0$. However, for the majority of cases, the observation time, $t$, is much greater than $\tau$, and accordingly it is possible to let $\tau \approx 0$. Under these conditions, Equations (A-13) and (19) yield Equation (20) (assuming that $\left.N_{0}=\zeta N_{t o t}\right)$, which has been previously used for estimating the number of stable pits nucleated under constant external conditions.

\section{Issues and Concerns: $\quad$ None}

\section{III-5.3 Developing a Relationship Between Deterministic and Statistical Methods for Predicting the Progression of Corrosion Damage}

In this section of the project, we explore and develop the relationship that exists between deterministic and statistical methods for predicting the progression of corrosion damage. Our objective is to show mathematically that there exists a close correspondence between Damage Function Analysis (DFA), which has been described at some length in the first part of this paper and Extreme Value Statistics (EVS). The latter technique has been used extensively to extrapolate damage (maximum pit or crack depth) from small samples in the laboratory to larger area samples in the field. Furthermore, DFA provides a means of calculating the central and scale parameters and their timedependencies in EVS from first principles and hence represents a unification of the two philosophies.

\section{$\underline{\text { Task Status }}$}

From the statistical point of view, all distributed properties of the system are completely determined by a Cumulative Distribution Function (CDF), $\Phi(x)$. By definition, $\Phi(x)$ is the probability that the depth of a randomly selected pit is $\leq x$. We will postulate that the pit distribution on the metal surface is uniform. Accordingly, the total number of nucleated pit in the whole system is $\mathrm{SN}(\mathrm{t})$, where $\mathrm{S}$ is the area of the system and, from the definition of the integral damage function, $\mathrm{F}$, the number of pits that have the depth $\leq x$, is $\mathrm{S}[\mathrm{N}(\mathrm{t})-\mathrm{F}(\mathrm{x}, \mathrm{t})]$. Accordingly, by definition of probability we have 


$$
\Phi(x, t)=\frac{S[N(t)-F(x, t)]}{S N(t)}=1-\frac{F(x, t)}{N(t)}
$$

We see that the cumulative distribution function for a given observation time, $\Phi(x, t)$, can be calculated if we know (can calculate) the integral damage function of the system [note that the number of nucleated stable pits, $N(t)$ simply equals $F(0, t)$ ]. This relation can be regarded as being the bridge between the statistical and deterministic approaches for estimating the accumulation of localized corrosion damage on a surface.

Let us calculate, for example, the failure probability, $\mathrm{P}_{\mathrm{f}}$, of a system. By definition, $\mathrm{P}_{\mathrm{f}}$, is the probability that at least one corrosion event in any form (pit, crevice, stress corrosion crack, or fatigue crack) reaches a depth, $\mathrm{x}$, at a given observation time, $\mathrm{t}$, where $\mathrm{x}$, in this case, is the critical dimension. It is evident that the probability of a corrosion event not achieving the depth $x$ at the tine, $\mathrm{t}$, is $\Phi(x, t)$. Accordingly, the probability that none of the corrosion events will reach the depth of $x$ is, $\Phi(x, t)^{S N(t)}$ where $S$ is the surface area of the system and, finally, the probability of failure is

$$
P_{f}(x, t)=1-\{1-F(x, t) / N(t)\}^{S N(t)}
$$

Using the well known limit relation, $\lim (1+a / x)^{x} \rightarrow \exp (a)$ as $x \rightarrow \infty$, and by assuming that $\mathrm{F}(\mathrm{x}, \mathrm{t}) / \mathrm{N}(\mathrm{t})<<1$ and $S N(t)$ is a large number, we can rewrite Equation (26) in the form

$$
P_{f}(x, t)=1-\exp \{-S F(x, t)\}
$$

As has been shown experimentally, in many practical cases, the asymptotic behavior (for large values of $x$ ) of the CDF can be described by the exponential relationship $[8,23]$.

$$
\Phi(x, t)=1-\exp [-(x-u) / \alpha]
$$


where $\mathrm{u}$ is the central parameter (the most frequent value) and $\alpha$ is the scale parameter, which defines the width of the distribution.. Accordingly, as follows from Equation (28), the extreme value distribution (EVD), $\Psi(x, t)$ (the probability that the largest value of pit depth $\leq x)$ is described by a double exponent (Gumbel Type I extreme value distribution) in the form [22].

$$
\Psi(x, t)=1-P_{f}=\exp [-\exp (-y)]
$$

where

$$
y=(x-h) / \alpha
$$

and $h=u+\alpha \ln S$.

In many applications, the Type 1 distribution has been claimed to account for the statistical nature in the observed behavior of corrosion systems [8,9,21,23]. Specifically, the Type I extreme value distribution is used for extrapolating corrosion damage measured on a series of small samples of area, $s$, to a large system (for example, tanks or pipe lines) with area, $S$ [24]. In this case, the probability of failure can be estimated from the relation

$$
P_{f}(x, t)=1-\exp \{-\exp [-(d-[h+\alpha \ln (S / s)]) / \alpha)]
$$

where $d$ is the wall thickness and parameters $h$ and $\alpha$ are measured by using the small samples.

It is interesting to determine whether Damage Function Analysis (DFA) can predict the behavior of the extreme value distribution. For simplicity, we will consider the limiting, but real, case when the observation time is much longer than the induction time for the majority of nucleated stable pits ("instantaneous" nucleation conditions). Experimentally determined extreme value distributions for pits have been reported for these conditions [9]. In addition, we also assume that the pit propagation rate is so low 
that it can be considered to occur at a constant rate, $V$. Under these conditions, as follows from Equations (16), (17), and (25), the Cumulative Distribution Function is given by

$$
\Phi(x, t)=1-\exp (-\gamma x / V) U_{+}(t-x / V)
$$

As noted by many sources $[8,21]$, an important characteristic of the unlimited CDF [see Equation (28)] is that it implies a small, but finite, probability of observing a pit of exceptional depth. The CDF given by Equation (28) was obtained from a conservation law and naturally it is free from that weakness (there are no pits for $x>V t$ ). For $x \leq V t$ the CDF given by Equation (32) coincides with the CDF described by Equation (28), if we choose $\alpha=V / \gamma$ and $u=0$, i.e. parameters $\alpha$ and $u$ do not depend on time. However, experiment shows that parameters $\alpha$ and $u$ are generally functions of time, and, particularly, the central parameter, $u$ is positive [7-9]. Moreover, for short periods of time, we can neglect the repassivation of pits, i.e. let $\gamma=0$ in Equation (32). In this case, Equation (32) reduces to a step function. However, experimentally observed extreme value distributions with $u>0$ for systems where the period of time over which damage has developed is of order of $10^{2}-10^{3}$ hours [7], i.e. for periods that are much lower than the service life, $t_{\mathrm{s}}$, of many practical systems, leaves little doubt that a distribution exists in the cavity growth rate, as alluded to earlier in this paper.

Because, under these limiting conditions, we neglect the nucleation stage of pit life ("instantaneous" nucleation) and because we also neglect the repassivation of pits, the only explanation of the inconsistency noted above must arise from the propagation of pits. In other words, Equation (7) cannot be used, in the general case, for describing the flux density of pits. It has been tacitly assumed above that the rate of pit propagation is unequivocally determined by its depth and by the external conditions, i.e. there is no distribution in pit propagation rate for pits with equivalent depth. However, it is well known that the morphology of pits on any given surface can vary significantly, with some shapes favoring more rapid mass and charge transfer, and hence greater propagation rate [8]. In addition, some pits will initiate at metallurgical features that may favor more rapid propagation, e.g. MnS inclusion [8]. The distribution in pit propagation rate might 
also be explained, for example, by the spatial distribution in electrochemical activity of the anodic and cathodic sites on the corroding surface [7].

In this article [7], we will assume, for simplicity, that the pit propagation rate does not change significantly during the propagation stage. This assumption can be justified for many real systems [20]. In generalizing DFA, for this case, we assume that the pits that propagate at a constant rate, $\mathrm{v}$, nucleate in accordance with the equation.

$$
n(t)=\int_{0}^{\infty} \lambda(t, v) d v
$$

Here, the function $\lambda(t, v)$ yields the number of pits with propagation rates between $v$ and $v+d v$, that nucleate in the period of time between $t$ and $t+d t\left(\right.$ per $\left.\mathrm{cm}^{2}\right)$. For the total number of nucleated pits, we have by analogy

$$
N(t)=\int_{0}^{\infty} \Lambda(t, v) d v
$$

where $\Lambda(t, v)$ yields the total number of pits with propagation rates between $v$ and $v+d v$ that are nucleated during the observation time, $t,\left(\right.$ per $\left.\mathrm{cm}^{2}\right)$. Finally, it is evident that

$$
\Lambda(t, v)=\int_{0}^{t} \lambda(\tau, v) d \tau
$$

Equation (7), for the flux density of active pits in the absence of the distribution in pit propagation rate, together with $V(x) \approx$ constant, can be rewritten in the following form [see Equation (12)]

$$
j_{a}(x, t)=f_{a}(x, t) V(x)=\exp (-\gamma \theta) n(t-\theta)=\exp (-\gamma x / V) n(t-x / V)
$$


and hence, when a distribution in the pit propagation rate is present, this expression can be generalized as

$$
j_{a}(x, t)=\int_{0}^{\infty} \exp (-\gamma x / v) \lambda(t-x / v, v) d v
$$

Under these conditions, the solutions of transport Equation (3) have the form

$$
\begin{aligned}
& f_{a}=\int_{0}^{\infty} \frac{\exp (-\gamma x / v) \lambda(t-x / v, v)}{v} d v \\
& f_{p}=\int_{0}^{\infty} \frac{\gamma \exp (-\gamma x / v) \Lambda(t-x / v, v)}{v} d v
\end{aligned}
$$

and

$$
F=\int_{0}^{\infty} \exp (-\gamma x / v) \Lambda(t-x / v, v) d v
$$

Let us assume that the distribution in pit propagation rate does not depend on time, i.e.

$$
\lambda(t, v)=n(t) \psi(v)
$$

and, correspondingly,

$$
\Lambda(t, v)=N(t) \psi(v)
$$

where the function, $\psi(v)$, satisfies the evident normalization condition 


$$
\int_{0}^{\infty} \psi(v) d v=1
$$

Accordingly, we have for the integral damage function and the cumulative damage function the following expressions

$$
F(x, t)=\int_{0}^{\infty} N(t-x / v) \exp (-\gamma x / v) \psi(v) d v
$$

and

$$
\Phi(x, t)=1-\int_{0}^{\infty} N(t-x / v) \exp (-\gamma x / v) \psi(v) d v / N(t)
$$

respectively. In particular, for the case of instantaneous nucleation, we have

$$
\Phi(x, t)=1-\int_{x / V}^{\infty} \exp (-\gamma x / v) \psi(v) d v
$$

To move further, we must assume a distribution function, $\psi(v)$, for the pit growth rate. For our purposes, it is most convenient to approximate $\psi(v)$, by Laplace's distribution function [25]

$$
\psi(\mathrm{v})=\frac{\exp (-|\mathrm{v}-\mathrm{V}| / \beta)}{2 \beta}
$$

with the mean pit propagation rate, $\mathrm{V}$, and dispersion $\sigma^{2}=2 \beta^{2}$. Strictly speaking, Distribution (47) satisfies the normalization condition $\int_{-\infty}^{\infty} \psi(v) d v=1$, but not Condition (43). The proper form of Distribution (47) that satisfies Conditions (43) is 


$$
\psi(\mathrm{v})=\frac{\exp (-|\mathrm{v}-\mathrm{V}| / \beta)}{2[\beta-\exp (-\mathrm{V} / \beta)]}
$$

However, for $V / \beta \geq 2$, Distributions (47) and (48) practically coincide.

Generally, values for the kinetic parameters $V$ and $\beta$ (along with parameters that described pit nucleation and repassivation) can be found from independent experiments or might even be estimated from first principles. In any event, all unknown parameters can also be determined by fitting Equations (44) or (27) to the experimentally measured CDF or EVD functions, as is customary, by applying a purely statistical approach. However, the application of DFA for estimating localized corrosion damage has real advantages over the purely statistical approaches. Thus, DFA yields the connection between the $\mathrm{CDF}$ and the physical parameters that determine the accumulation of damage. This connection allows us to reduce the number of fitting parameters for the $\mathrm{CDF}$, to predict the time dependencies of these parameters, and to predict the dependencies of some fitting parameters on the external conditions (corrosion potential, temperature, electrolyte composition, etc.).

As an example, let us consider the extrapolation of corrosion damage into the future for the system that has been investigated in Ref. 7: corrosion of manganese steel in $\mathrm{CO}_{2}-$ acidified seawater. Figure III-5.1 shows experimental extreme value distributions for pit depth measured for six different times (from $t_{1}=336$ hours to $t_{6}=1176$ hours). We will assume that we know nothing about the kinetic parameters for this system. Nevertheless, we will try to predict the propagation of the damage into the future by using only data obtained at $t=t_{1}$ and by using only the simplest, qualitative assumption concerning the damage propagation process. 


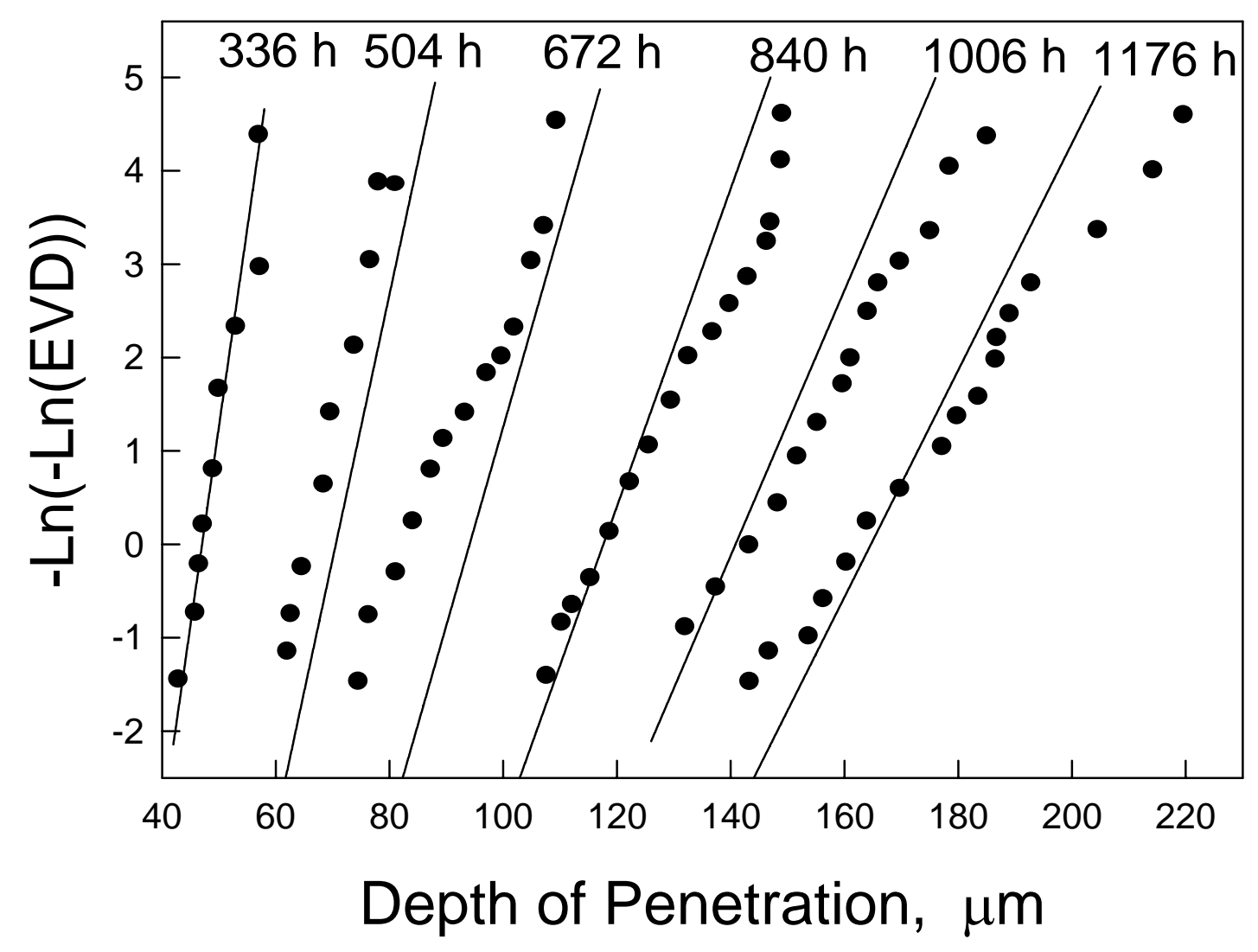

Figure III-5.1 Examples of predicted damage functions in pit depth for the pitting corrosion of aluminum in chloride solutions for $E_{\text {corr }}=-0.384 \mathrm{~V}_{\text {she }},\left[\mathrm{Cl}^{\top}\right]=0.002 \mathrm{M}, p H=7$, and $\gamma=3$ year [1].

First of all, we assume that the induction time for pit nucleation is much smaller than $t_{l}$ and, accordingly, that pit nucleation can be regarded as being instantaneous. This assumption is reasonable, if the environment is reasonably aggressive (high chloride concentration) and the exposure time is large, as is the case in this example. For example, many $\mathrm{Fe}-\mathrm{Cr}$ alloys display pit induction times of the order of $10^{2} \mathrm{~s}$ for commonly encountered environmental conditions [14]. We will also assume that $t_{1}$ is much smaller than the service life for normal structures made from this steel, and, accordingly, it is possible to neglect pit repassivation for $t \leq t_{1}$.

Substitution of Equation (47) into Equation (44) with $N(t)=N_{0} U_{+}(t)$ (instantaneous nucleation) and $\gamma=0$ (no pit repassivation) yields: 


$$
F(x, t)=N_{0}\left[1-\frac{1}{2} \exp \left(\frac{x / t-V}{\beta}\right)\right] \quad \text { for } x \leq V t
$$

and

$$
F(x, t)=\frac{N_{0}}{2} \exp \left(\frac{V-x / t}{\beta}\right) \quad \text { for } x>V t
$$

Because we are interested in the behavior of the CDF for large values of $x$, we obtain the following expression for the EVD

$$
\Psi(x, t)=1-P_{f}=\exp \left[-\frac{S N_{0}}{2} \exp \left(\frac{V-x / t}{\beta}\right)\right]
$$

which absolutely coincides with the Type I extreme value distribution [Equations (29) and (30)] if we choose

$$
\alpha=\beta t \quad \text { and } \quad h=\left[V+\beta \ln \left(0.5 S N_{0}\right)\right] t
$$

The reader will note that Equation (51) does not contain parameters related to the nucleation of damage or to delayed repassivation, because we have assumed instantaneous nucleation conditions and we postulated that (for this particular case) delayed repassivation was not important. However, in the general case, both assumptions are invalid, and hence it is necessary to use Equation (45) to derive the extreme value distribution function. These calculations can only be performed numerically, in the general case, and their use in deriving extreme value distribution functions will be illustrated in a subsequent paper.

Proceeding now with our analysis of the pitting of manganese steel in $\mathrm{CO}_{2}$ acidified seawater, we see that fitting the scale parameter, $\alpha$, and the central parameter, $h$, from Equations (29) and (30) to the experimental data for $t=336$ hours yields $\alpha=3.7 \mu \mathrm{m}$ and $h=50 \mu \mathrm{m}$. In accordance with Equation (52), we have $\beta=0.011 \mu \mathrm{m} / \mathrm{h}$ and $V+$ $\beta \operatorname{Ln}\left(0.5 S N_{0}\right)=0.15 \mu \mathrm{m} / \mathrm{h}$. Using these values for the parameters, we calculated EVDs 
for different times assuming that, even for $t_{6}=1176$ hours, pit repassivation is not important. Satisfactory agreement between the experimental and predicted data is observed (Figure III-5.1).

DFA allows us to explore the influence of repassivation on the time-dependencies of parameters $\alpha$ and $h$. If the distribution in propagation rate is sufficiently sharp $(V>>\beta)$, Laplace's distribution can be considered to be an approximation of the $\delta$ function. Under these conditions, for the case of instantaneous nucleation, we have from Equation (44):

$$
F(x, t)=N_{0} \int_{x / V}^{\infty} \exp (-\gamma x / v) \psi(v) d v \approx N_{0} \exp (-\gamma x / V) \int_{x / V}^{\infty} \psi(v) d v
$$

By analogy with Equation (52), it is easily shown that parameters $\alpha$ and $h$ can be expressed as

$$
\alpha=\frac{\beta t}{1+\gamma \beta t / V} \text {, and } h=\frac{\left[V+\beta \operatorname{Ln}\left(0.5 S N_{0}\right)\right] t}{1+\gamma \beta t / V}
$$

We see that, for small values of $t$, the scale and central parameters can be described by Equation (52), i.e. they are proportional to time. However, for large observation times, parameters $\alpha$ and $h$ go to limits that are independent of time. This fact can be regarded as being physically evident, because at sufficiently large times all pits become passive (the damage function becomes "frozen") and further propagation of damage cannot occur, provided that $\gamma>0$. This conclusion concerning the achievement of limiting pitting depth also flows from a formal statistical treatment of the experimental data for underground pipelines made from carbon steels [4]. The "freezing" of damage functions can also be seen in the propagation of corrosion damage on aluminum in tap water [9].

In the more general case, we suggest the following, simple expressions for representing the time dependencies of the scale and central parameters 


$$
\alpha=\frac{\alpha_{1} t}{1+\left(\alpha_{1} / \alpha_{\infty}\right) t} \text {, and } h=\frac{h_{l} t}{1+\left(h_{1} / h_{\infty}\right) t}
$$

For small times, Equations (55) yields the linear dependencies, $\alpha=\alpha_{1} t$ and $h=h_{1} t$, and for large times it yields the limiting values of $\alpha=\alpha_{\infty}$ and $h=h_{\infty}$. It is evident that experimental data for at least two times are required for determining the unknown parameters $\alpha_{1}, h_{1}, \alpha_{\infty}$ and $h_{\infty}$. Figure III-5.2 shows that Equations (55) can be used for extrapolating experimental data from Ref. 9 for the pitting of aluminum in tap water to the future. It is interesting to note that Finley [26] suggested that the experimental data for the pitting corrosion of aluminum in tap water (from Ref. 9) could be described by assuming that $\alpha$ does not change with time and that $h$ could be described by the logarithmic relation

$$
h=b \operatorname{Ln}(t)+c
$$

However, it is evident that Equation (56) cannot describe the experimental data for small values of $t$, when the condition $h \rightarrow 0$ for $t \rightarrow 0$ must be fulfilled, and for large values of $t$ when $h$ must go to a constant value. It should be emphasized that the power law (see Ref. 3) cannot describe the cessation of localized corrosion damage due to pit passivation. That is why, in our opinion, it is much more appropriate to use a hyperbolic law than to employ a logarithmic law (and power law) for representing the time dependencies of the EVS parameters. 


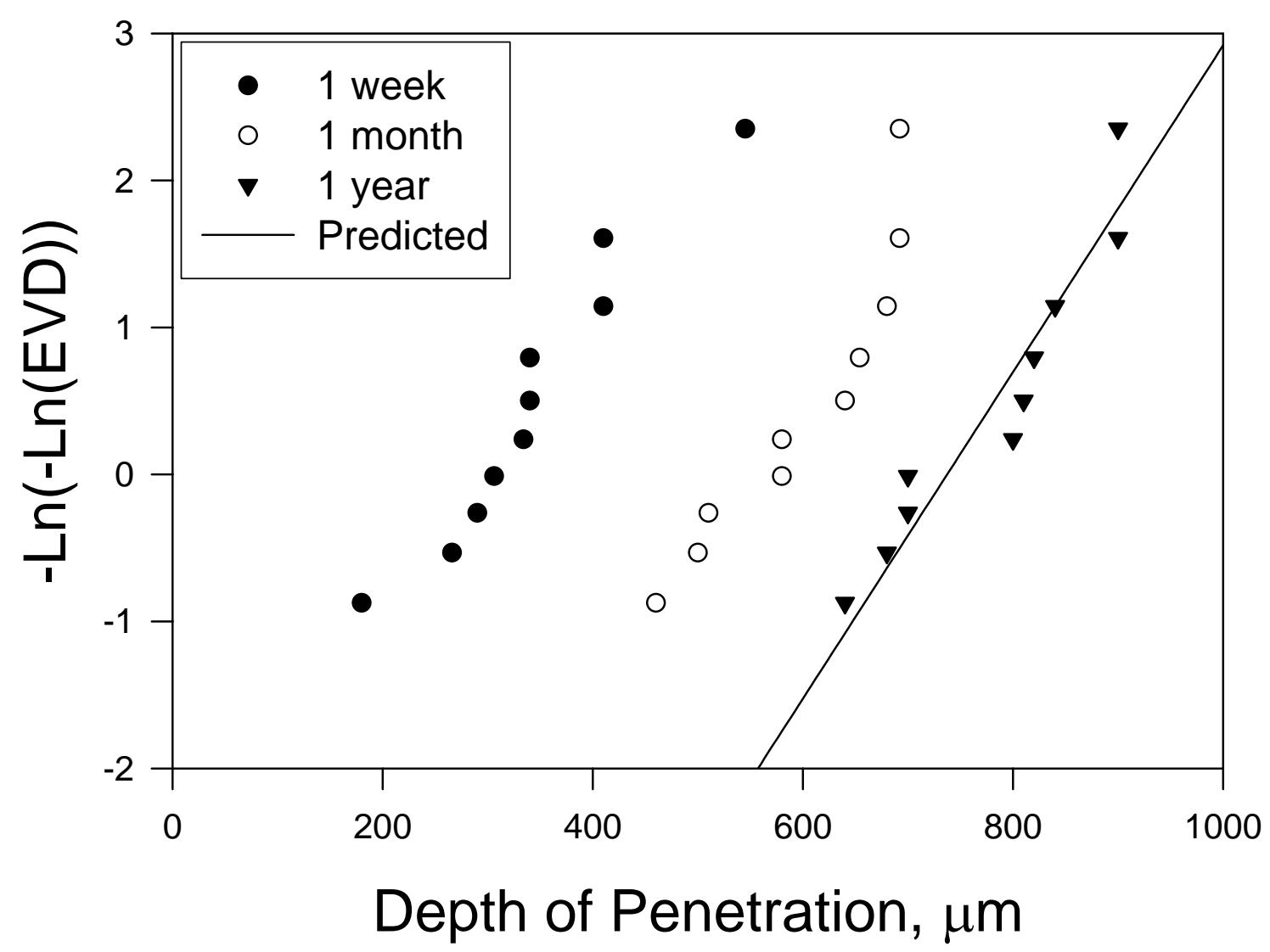

Figure III-5.2 Extreme value distribution functions (EVDs) versus exposure time for the pitting of manganese steel in CO2-acidified seawater. Only the data for $\mathrm{t}=336$ hours were used for fitting, in order to determine the central and scale parameters. Experimental data are taken from Figure 5 in Ref. 7.

It is important to note that the Gumbel asymptotic extreme (Type I) can be used for describing the statistics of localized corrosion damage when repassivation (death) is considered, even though nucleation is instantaneous (Figure III-5.3) or even for the case of progressive nucleation where two different nucleation functions have been considered (Figures III-5.4, III-5.5). Note that Figure III-5.4 shows the EVD for the nucleation rate function for the deterministic Point Defect Model. Numerical calculation shows that, in all of these cases, the dependencies of $\operatorname{Ln}(1-C D F)$ on pit depth, $x$, can be approximated by straight lines, at least for sufficiently large values of $x$. The work presented in this paper, therefore, provides a theoretical basis for applying Type I extreme value distributions for real corrosion systems. 


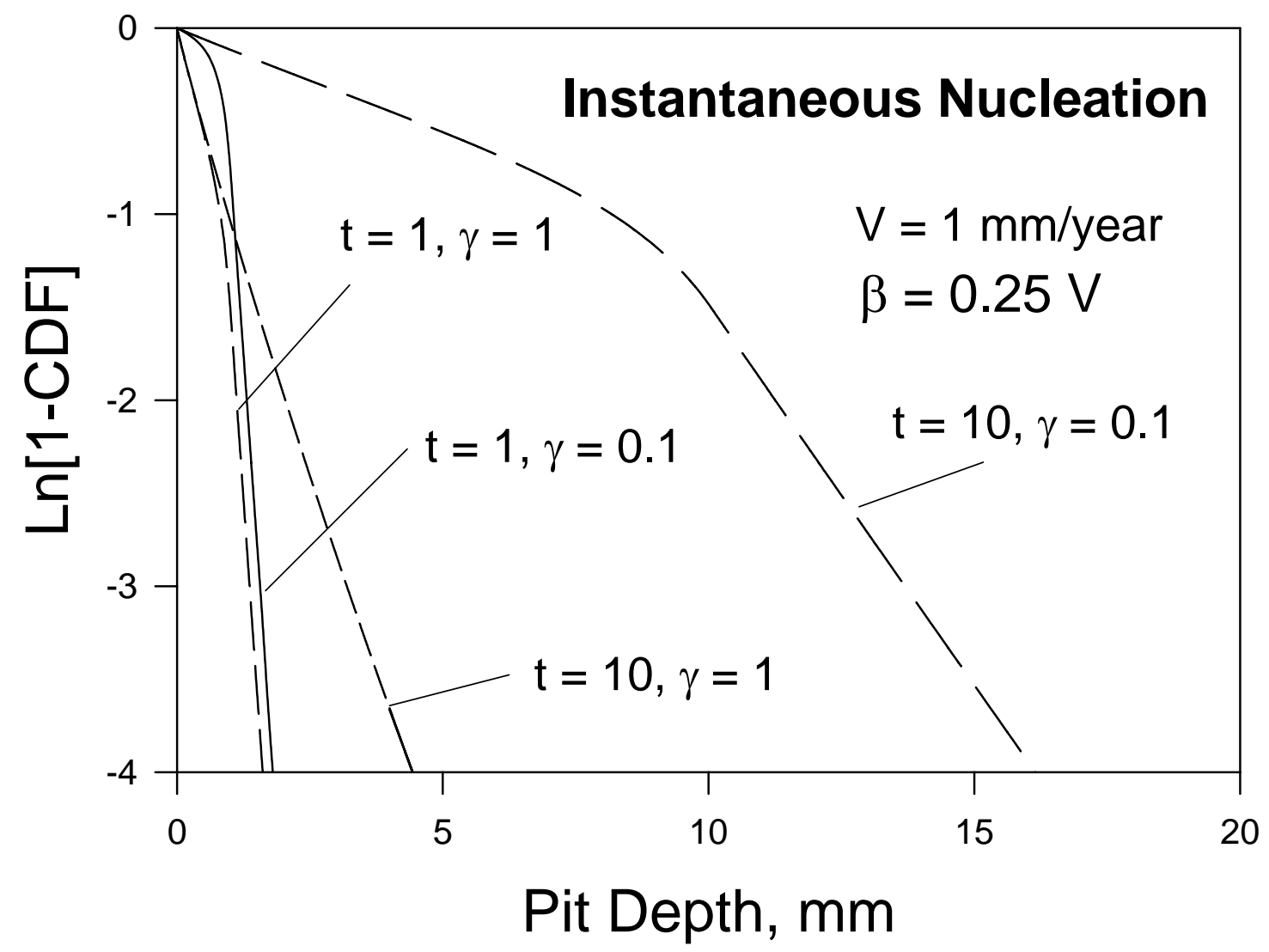

Figure III-5.3 Variation of the cumulative distribution function (CDF) with pit depth as a function of time for instantaneous nucleation. Observation times, $t$, are measured in years and the delayed repassivation constant, $\gamma$, is given in units of year [1]. 


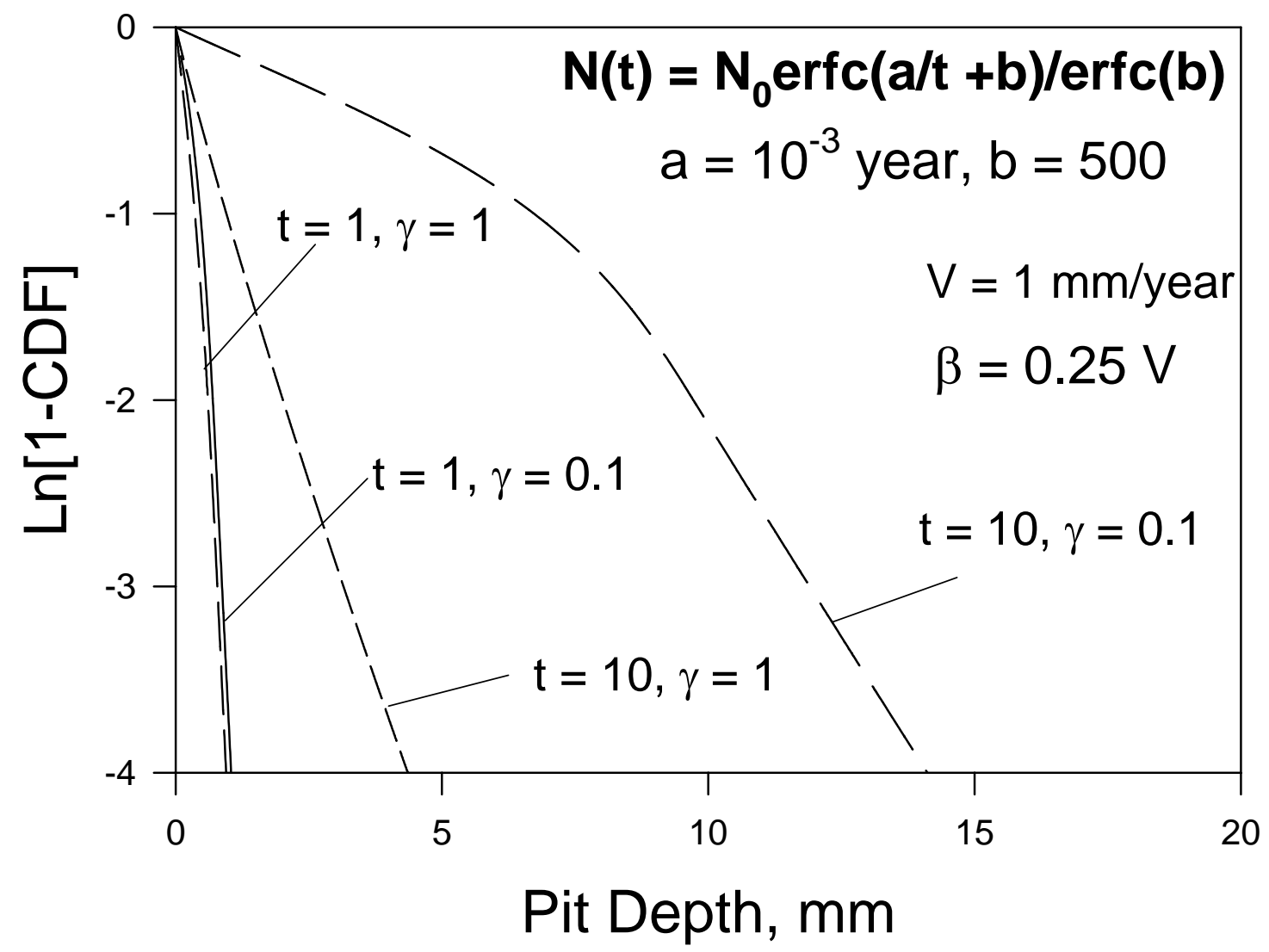

Figure III-5.4 Variation of the cumulative distribution function (CDF) with pit depth as a function of time for the case when the nucleation of pits is described by the Pont Defect Model. Observation times, $t$, are measured in years and the delayed repassivation constant, $\gamma$, is given in units of year [1]. 


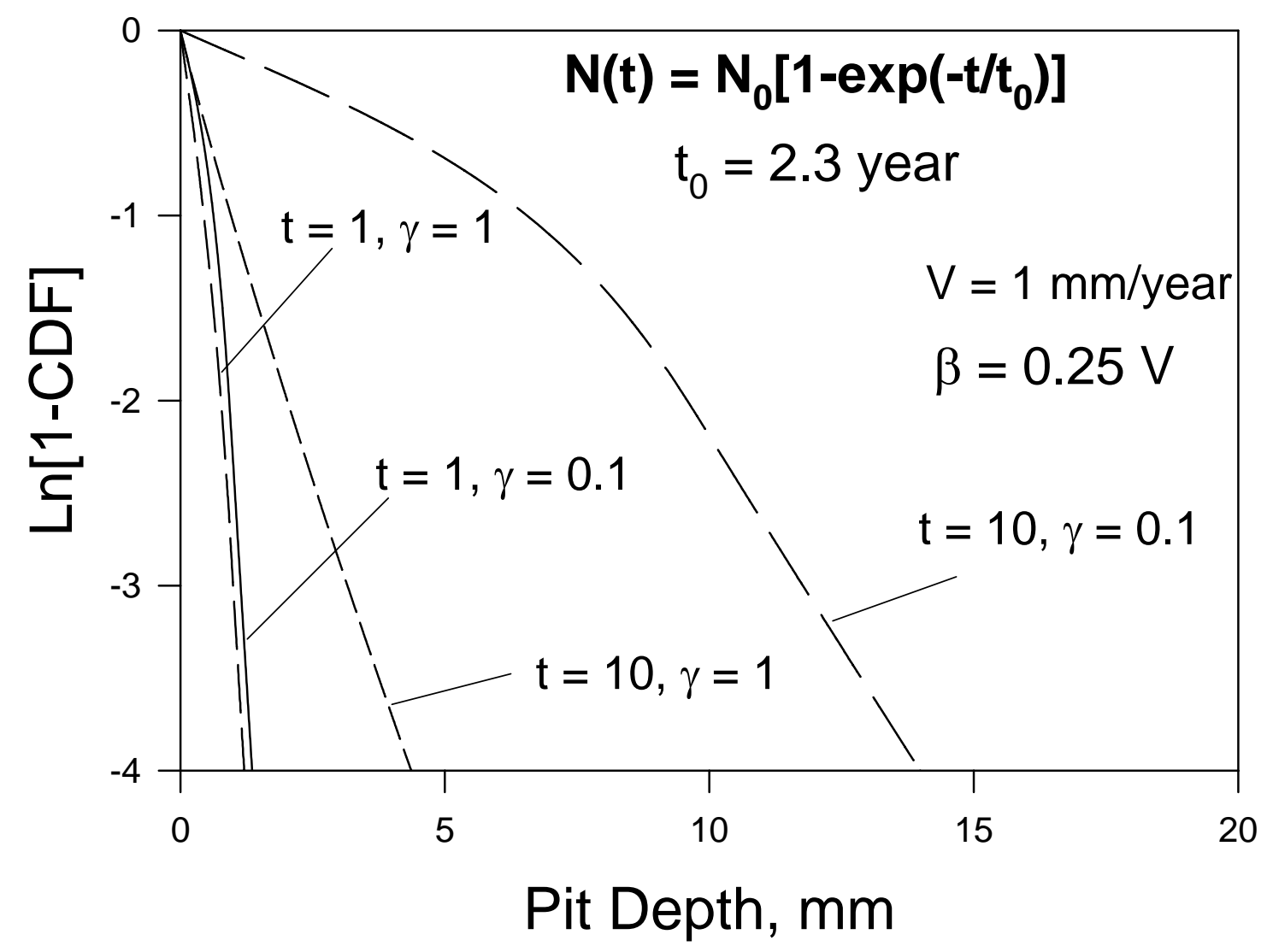

Figure III-5.5 Variation of the cumulative distribution function (CDF) with pit depth as a function of time for the case when the nucleation of pits is described by an exponential function. Observation times, $t$, are measured in years and the delayed repassivation constant, $\gamma$, is given in units of year [1].

\section{Issues and Concerns: $\quad$ None}

\section{III-5 References}

1. D. D. Macdonald and M. Urquidi-Macdonald, Corrosion, 48, 354 (1992).

2. D. D. Macdonald, C. Liu, M. Urquidi-Macdonald, G. H. Stickford, B. Hindin, A. K. Agrawal, and K. Krist, Corrosion, 50, 761 (1994).

3. P. J. Laycock, R. A. Cottis and P.A. Scarf, J. Electrochem. Soc., 137, 64 (1990).

4. L. I. Freiman. Progress in Science and Technology. Corrosion and Corrosion Protection [in Russian], 11, VINITI, p. 3, (1985).

5. G. Engelhardt, and D. D. Macdonald, Corrosion, 54, 469 (1998).

6. G. Engelhardt and D. D. Macdonald. "The Deterministic Prediction of Localized Corrosion Damage in Aircraft Aluminum Alloys," in 1998 USAF Aircraft Structural Integrity Program Conference, 1- 3 December 1998, San Antonio, Texas. 
7. J. E. Strutt, J. R. Nicholls and B. Barbier, Corros. Sci., 25, 305 (1985).

8. G. P. Marsh, I.D. Bland and K. J. Tailor, Br. Corr. J., 23, 157 (1988).

9. P. M. Aziz, Corrosion, 12, 495 (1956).

10. P.S. Pistorius and G.T. Burstein, Phil. Trans. R. Soc. Lond. A, 341, 531 (1992).

11. J. Stewart and D. Williams. "Studies of the initiation of pitting corrosion on stainless steels", in Advances in Localized Corrosion (ed. H. S. Isaacs et al.) NACE-International, Houston, Texas, pp.131-136 (1990).

12. D. Williams, J Stewart and P. H. Balkwill, Corr. Sci. 36, 1213 (1994).

13. L. F. Lin, C. Y. Chao, and D. D. Macdonald, J. Electrochem. Soc., 128, 1194 (1981).

14. D. D. Macdonald and M. Urquidi-Macdonald, Electrochim. Acta, 31, 1079 (1986).

15. D. D. Macdonald and M. Urquidi-Macdonald, J. Electrochem. Soc., 134, 41 (1987).

16. D. D. Macdonald and M. Urquidi-Macdonald, J. Electrochem. Soc., 136, 961 (1989).

17. D. D. Macdonald and M. Urquidi-Macdonald, J. Electrochem. Soc., 139, 3434 (1992).

13. L. F. Lin, C. Y. Chao, and D. D. Macdonald, J. Electrochem. Soc., 128, 1194 (1981).

14. D. D. Macdonald and M. Urquidi-Macdonald, Electrochim. Acta, 31, 1079 (1986).

15. D. D. Macdonald and M. Urquidi-Macdonald, J. Electrochem. Soc., 134, 41 (1987).

16. D. D. Macdonald and M. Urquidi-Macdonald, J. Electrochem. Soc., 136, 961 (1989).

17. D. D. Macdonald and M. Urquidi-Macdonald, J. Electrochem. Soc., 139, 3434 (1992).

18. Z. Szklarska-Smialowska, Pitting Corrosion of Metals, NACE-International, Houston, 1986.

19. G. R. Engelhardt, D. D. Macdonald, and M. Urquidi-Macdonald, Corros. Sci., 41, 2267 (1999).

20. G. Engelhardt and D. D. Macdonald, Corr. Sci., (2004). In press.

21. G. E. Eldredge, Corrosion, 13, 51 (1957)

22. E. J. Gumbel, Statistics of Extremes, Columbia University Press, NY, (1957).

23. A. Turnbull, Br. Corros. J., 28, 297 (1993).

24. T Shibata et al., Corros. Eng., 37, 699 (1988).

25. A. Korn and M. Korn, "Mathematical Handbook", McGraw-Hill, NY, (1968).

26. H. F. Finley, Corrosion, 23, 83 (1967).

27. T. Haruna and D. D. Macdonald, J. Electrochem. Soc., 144, 1574 (1997).

28. I. Fonseca, N. Lima, J. A. Rodrigues, M. I. S. Pereira, J. C. S. Salvador, M. G. S. Ferreira, Electrochemistry Communications, 4, 353 (2002).

29. Handbook of Physics and Chemistry, (Edit. R. C. Weast), CRC Press, Boca Raton, Fl, $59^{\text {th }}$ Edit., $1978 / 79$.

30. D. Ellerbrock and D. D. Macdonald, Mater. Sci. Forum, 185-188, 927 (1995).

31. S. Ahn, H-S. Kwon, and D. D. Macdonald, "The Role of Chloride Ion in Passivity Breakdown on Nickel", Proc. Symp. Surface Oxide Films, Electrochemical Society, Orlando, FL, Oct. 12-17, 2004, in press.

32. T. Shibata, Trans ISIJ, 23, 785 (1983).

33. R. Fratesi, Corrosion, 41, 114 (1985). 


\section{IV.Publications in Peer Reviewed Journals and Conference Proceedings}

1. J. Liu and D. D. Macdonald, "The Passivity of Iron in the Presence of Ethylenediaminetetraacetic Acid II. The Defect and Electronic Structures of the Barrier Layer", J. Electrochem. Soc., 148 (11), B425-B430 (2001)

2. Vankeerberghen, M., and D. D. Macdonald, "Predicting Crack Growth Rate vs. Temperature-Behaviour of Type 304 Stainless Steel in Dilute Sulfuric Acid Solutions", Corros. Sci., 44, 1425-1441 (2002).

3. Macdonald, D. D., et.al., "High temperature on-line monitoring of water chemistry and corrosion control in water cooled power reactors; Report of a coordinated research project 1995-1999", Proc. IAEA-TECDOC-1303, International Atomic Energy Agency (IAEA), Vienna, Austria, July 2002.

4. Liu, S. and D. D. Macdonald, "Fracture of AISI 4340 Steel in Concentrated Sodium Hydroxide Solution", Corrosion, 58, 835-845 (2002).

5. Sikora, E. and D. D. Macdonald, "Nature of the Passive Film on Nickel", Electrochimica Acta, 48, 69-77 (2002).

6. Macdonald, D. D., "Comments on 'Electrochemical Transients During the Initial Moments of Anodic Oxidation of Aluminum' by Huiguan Wu and Kurt Hebert, Electrochimica Acta, 47, 1373-1383 (2002)", Electrochimica Acta, 48, 127-130 (2002)

7. Macdonald, D. D., "Controlling IGSCC in BWR Coolant Circuits", Proc. 2nd ${ }^{\text {st }}$ DAWAC Conf., Int. Atomic Energy Agency, Prague, Czech Republic, Nov. 26-29, (2002)

8. Macdonald, D. D. and G. R. Engelhardt, "Deterministic Prediction of Localized Corrosion Damage - A Reflective Review of Critical Issues", J. Corros. Sci. Engin., 6, Paper C066 (2003).

9. Macdonald, D. D., "Internal/External Environment Coupling in Stress Corrosion Cracking”, J. Corros. Sci. Engin., 6, Paper C065 (2003).

10. Vankeerberghen, M. and D. D. Macdonald, "Calculating the Temperature Maximum and the Lower Potential Limit for the Crack Growth Rate in Type 304 SS Using the CEFM", Paper 03250, CORROSION2003, San Diego, CA, March 2003.

11. Macdonald, D. D. and Gomez-Duran, M., "Stress Corrosion Cracking of Sensitized Type 304 Stainless Steel in Thiosulfate Solution: I. Fate of the Coupling Current", Corrosion Science, 45, 1455-1471 (2003)

12. Englehardt, G. and D. D. Macdonald, "Estimation of Corrosion Cavity Growth Rate for Predicting System Service Life", Corrosion Science, 46(5), 1159-1187 (2004).

13. Englehardt, G. and D. D. Macdonald, "Unification of the Deterministic and Statistical Approaches for Predicting Localized Corrosion Damage: I. Theoretical Foundation", Corrosion Science, in press (2003). 
14. Macdonald, D. D., "Passive Films: Nature's Exquisitely Nano-Engineered Protection System”, Current Applied Physics, 4(2-4), 129-132 (2004).

15. Macdonald, D. D. and G. Englehardt, "Theory For The Deterministic Prediction Of Pitting Corrosion Damage On Aluminum”, Proc. Electrochemical Society, Orlando, FL, Oct. 12-17, 2003.

16. Ahn, S., HyukSang Kwon, and D. D. Macdonald, "The Role of Chloride Ion in Passivity Breakdown on Nickel", Proc. Electrochemical Society, Orlando, FL, Oct. 12-17, 2003.

17. Macdonald, D. D. and Gomez-Duran, M., "Stress Corrosion Cracking of Sensitized Type 304 Stainless Steel in Thiosulfate Solution: I. Fate of the Coupling Current", Corrosion Science, 45, 1455-1471 (2003)

18. Gomez-Duran, M. and Macdonald, D. D., "Stress Corrosion Cracking of Sensitized Type 304 Stainless Steel in Thiosulfate Solution: II. Dynamics of Fracture", Corrosion Science, submitted 2004.

19. Macdonald, D. D., "Critical Issues in Understanding Corrosion and Electrochemical Phenomena in Super Critical Aqueous Media”, Paper \#04484, CORROSION '04, New Orleans, LA, March 2004.

20. Macdonald, D. D., "After Two Decades of Predicting Crack Growth Rate - What Have We Learned?", Paper \#04673, CORROSION '04, New Orleans, LA, March 2004.

21. Soundararajan, B. and Macdonald, D. D., "Caustic Cracking of AISI 4340 Steel", Paper \#04572, CORROSION ’04, New Orleans, LA, March 2004.

22. Macdonald, D. D., "Fate of the Coupling Current in Stress Corrosion Cracking Mechanistic and Corrosion Control Implications", Paper \#04570, CORROSION '04, New Orleans, LA, March 2004.

23. Macdonald, D. D., "Fate of the Coupling Current in Stress Corrosion Cracking Mechanistic and Corrosion Control Implications", Paper \#04570, CORROSION '04, New Orleans, LA, March 2004

24. Macdonald, Digby D., "Stress Corrosion Cracking in Reactor Coolant Circuits - An Electrochemist's Viewpoint,” Power Plant Chemistry, 12, 731-747 (2004).

25. M. T. Smith and D. D. Macdonald, "Wavelet Analysis of Electrochemical Noise Data,” Paper \#05355, CORROSION 2005, Houston, TX, April 2005.

26. D. D. Macdonald, Predicting the Future from the Past in Corrosion Science and Engineering, Eurocorr 2004, Nice, France, Sept. 12-17, 2004. (Plenary). Paper in conference proceedings (will be published in 2005).

27. D. D. Macdonald and G. Engelhardt, Challenges in Predicting the Accumulation of Localized Corrosion Damage-A Review of Critical Issues, Egyptian Corrosion Soc., 23rd Conf. Corrosion Problems in Industry, Ras Sedr, Egypt, Dec. 6-9, 2004. (Invited) 


\section{Presentations}

1. D. D. Macdonald, "The Holy Grail: Deterministic Prediction of Corrosion Damage Thousands of Years into the Future", International Workshop on the Prediction of Long Term Corrosion Behavior in Nuclear Waste Systems", Chateau de Cadarache, France, Nov. 26-29, 2001.

2. D. D. Macdonald, “A Brief History of Electrochemical Impedance Spectroscopy”, Centenary Meeting of the Electrochemical Society, Philadelphia, PA, May 12-17, 2002. Invited.

3. D. D. Macdonald, "Recent Advances in the Theory of Stress Corrosion Cracking”, Institute of Nuclear Safety Systems, Osaka, Japan, May 26-30, 2002. Invited.

4. D. D. Macdonald, "Passivity, the Enabler of Our Metals-Based Civilization", University of Florida, July 12, 2002. Invited.

5. D. D. Macdonald, "Passivity and Passivity Breakdown", Georgia Institute of Technology, July 12, 2002. Invited.

6. D. D. Macdonald, "Can We Predict the Future in Corrosion Science?", 2002 DOE Corrosion Contractors Meeting, Brookhaven National Laboratory, September 1314, 2002.

7. D. D. Macdonald, "Can Corrosion Science Predict the Future?", presented in receipt of the U. R. Evans Award, British Corrosion Institute, Cardiff, Wales, October 23, 2002. Invited.

8. D. D. Macdonald, "Passive Films, Nature's Exquisitely Nano-Engineered Protection System", AMN-1, The Macdiarmid Institute for Advanced Materials and Nano Technology, Tepapa, Wellington, New Zealand, February 13, 2003. Invited.

9. M. Vankeerberghen and D. D. Macdonald, "Calculating the TemperatureMaximum and the Lower Potential Limit for the Crack Growth Rate in Type 304 SS Using the CEFM", CORROSION 2003, (NACE International, Houston, TX), San Diego, CA March 17-27, 2003.

10. D. D. Macdonald, "Mixed Potential-Point Defect Models for Nuclear Waste Containers", CORROSION 2003, (NACE International, Houston, TX), San Diego, CA March 17-27, 2003. Invited.

11. D. D. Macdonald, J. Liu, B. Marx, B. Soundararajan, M. Smith, and S. Ahn, "Development of Advanced Electrochemical Emission Spectroscopy for 
Monitoring Corrosion in Simulated DOE Liquid Waste", EMSP Worksop, Pacific Northwest National Laboratory, Richland, WA, May 6-8, 2003. Invited.

12. G. Engelhardt and D. D. Macdonald, "Prediction of the Accumulation of Localized Corrosion Damage to Low Pressure Steam turbines in Fossil Service", Seventh Intl. Conf. Cycle Chem. Fossil Plants, Houston, TX, June 3-5, 2003. Invited.

13. D. D. Macdonald, "Deterministic Prediction of Localized Corrosion", Intl. Symp. Corrosion Science in the $21^{\text {st }}$ Century, UMIST, Manchester, UK, July 6-11, 2003.

14. D. D. Macdonald, "Internal/External Environment Coupling in Stress Corrosion Cracking", Intl. Symp. Corrosion Science in the $21^{\text {st }}$ Century, UMIST, Manchester, UK, July 6-11, 2003. (Keynote Lecture).

15. D. D. Macdonald and G. R. Engelhardt, "Prediction of Long-Term Corrosion Damage in High Level Nuclear Waste Disposal Systems", Corrosion Workshop on Yucca Mountain Waste Repository", Department of Energy, Bethesda, MD, July 28-30, 2003. Invited.

16. D. D. Macdonald, G. R. Engelhardt, and Y. Zhang, "Prediction of Localized Corrosion Damage in Low Pressure Steam Turbines", EPRI, Charlotte, NC, August 11, 2003. Invited.

VI. Current Student Status

\begin{tabular}{|c|c|c|c|c|}
\hline Student Name & Date & Degree & Candidacy & $\begin{array}{c}\text { Expected } \\
\text { Started }\end{array}$ \\
& & Status & Graduation \\
\hline Brian M. Marx & $\mathbf{8 / 2 0 0 2}$ & M.S./Ph.D. & Passed- & $\mathbf{8} / \mathbf{2 0 0 5}$ \\
& & & $\mathbf{9 / 2 0 0 3}$ & \\
\hline Boundararajan & $\mathbf{8 / 2 0 0 2}$ & M.S./Ph.D & Passed- & $\mathbf{8} / \mathbf{2 0 0 6}$ \\
\hline Morgan Smith & $\mathbf{1 / 2 0 0 3}$ & M.S. & N/A & $\mathbf{1 2 / 2 0 0 4}$ \\
& & & & (graduated) \\
\hline
\end{tabular}

